\title{
The Thermalhydraulics of Tube-Support Fouling in Nuclear Steam Generators
}

\author{
by \\ Helena E.C. Rummens, B.Sc. (honors), M.Eng.

\begin{abstract}
A thesis submitted to
the Faculty of Graduate Studies and Research

in partial fulfillment of

the requirements for the degree of
\end{abstract} \\ Doctor of Philosophy \\ in Engineering \\ The Ottawa-Carleton Institute for \\ Mechanical and Aerospace Engineering \\ Department of Mechanical and Aerospace Engineering \\ Carleton University \\ Ottawa, Ontario \\ 1999 September \\ O copyright \\ 1999, Helena Rummens
}


National Library

of Canada

Acquisitions and Bibliographic Services

395 Wellington Street Otlawa ON K1A ONA Canada
Bibliothèque nationale du Canada

Acquisitions ef services bibliographiques

395, ne Wellington Ottawa ON KIA ONA Canada
The author has granted a nonexclusive licence allowing the National Library of Canada to reproduce, loan, distribute or sell copies of this thesis in microform, paper or electronic formats.

The author retains ownership of the copyright in this thesis. Neither the thesis nor substantial extracts from it may be printed or otherwise reproduced without the author's permission.
L'auteur a ascordé une licence non exciusive permettant à la Bibliothèque nationale du Canada de reproduire, prêter, distribuer ou vendre des copies de cette thèse sous la forme de microfiche/film, de reproduction sur papier ou sur format électronique.

L'auteur conserve la propriété du droit d'auteur qui protège cette thèse. $\mathrm{Ni}$ la thèse ni des extraits substantiels de celle-ci ne doivent être imprimés ou autrement reproduits sans son autorisation. 


\begin{abstract}
Nuclear steam generators (SGs) world-wide have experienced a wide variety of problems, of which a recent one has been fouling and blockage of the supports that restrain the SG primary-coolant tubes. Water chemistry and operating conditions are known to influence fouling, and it is hypothesized that the thermal and hydraulic environments near a support also play a role. The work presented here endeavours to show the effect of support design on this environment and hence on fouling.

Experiments were performed to simulate the thermalhydraulic environment near various designs of tube supports. Air/water mixtures were useful in showing the hydraulic flow patterns, while Freon-11 vapour/liquid mixtures showed thermal effects. Measurements of pressure loss, local velocity, and local void fraction were also made to quantitatively characterize the effect of the support.
\end{abstract}

A computer program, called TSFOUL, was coded to predict deposit thicknesses in and near a support. Larger codes used for such predictions in industry have been unable to predict blockage of supports, hence the need for support-specific models. TSFOUL has the same classic particle deposition models as in the larger codes, but considers additional factors such as stagnation zones and surfaces normal to the flow. The fouling mechanisms specific to supports were inferred from SG inspections and from experimental flow patterns, and measured values helped to make the models more quantitative. While limited by a lack of good validation data, TSFOUL was able to predict reasonable deposition patterns, and helped to understand the complex interaction between different mechanisms.

The net product is a set of tools for assessing the fouling propensity of a given tubesupport design: (1) proposed fouling mechanisms, (2) criteria for support fouling propensity, (3) correlation of fouling with mass flux and quality, (4) experimental tools 
such as flow visualization and measurement of pressure-loss profiles, and (5) analytical tools such as TSFOUL.

This work was part of a comprehensive program on SG fouling that involved a team of researchers at AECL's Chalk River Laboratories. This thesis focuses on the experiments and analytical modeling carried out by the author herself. 


\section{Acknowledgments}

The thesis is based on a project at my workplace (AECL at Chalk River Laboratories), funded over the 1991-1996 time period by CANDU Owner's Group R\&D Program, Working Party \#19 (Steam Generators), WPIR-1937. In addition to the work for the customer, $I$ independently undertook further analyses for the thesis.

AECL management generously supported (by partial financing) my leave of absence from work to complete the Ph.D. course-work in 1992-93, and my tuition. I am very grateful to the following individuals at AECL for their encouragement and for having risked the investment: Geoff Dimmick (section head), Alan Lane (former branch head), Paul Fehrenbach (general manager), and Dave Torgerson (vice-president).

Over the course of the thesis work, I have leaned heavily on four exemplary individuals:

My thesis supervisor, Terry Rogers, will always astound me with the depth and breadth of his technical knowledge. That, together with his patience, flexibility, and a good sense of humor, have made him a prime role model and a delight to work with.

My colleague at CRL, Carl Turner, has also been an excellent guide and endless source of information. He is magically able to discuss deposition theory while up to his ears in other projects.

My supervisor at CRL, Geoff Dimmick, was the one who first assigned this project to me, and allowed me-as usual- to make plenty of mistakes and learn.

Finally, my husband Bob Donders, a great critical thinker in his own field, made it possible to live a marvelously full life (sometimes too full) while I journeyed along the doctoral road. This thesis is dedicated to him, with the promise that I will monopolize the home computer less often from now on. 


\section{Table of Contents}

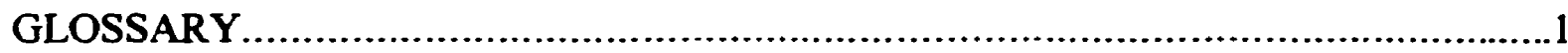

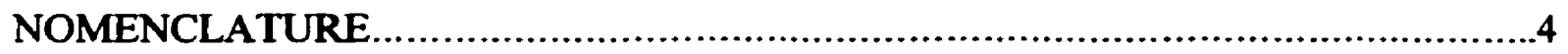

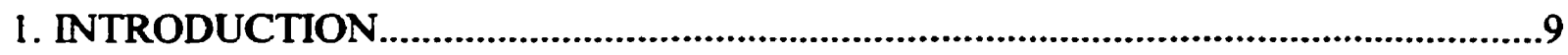

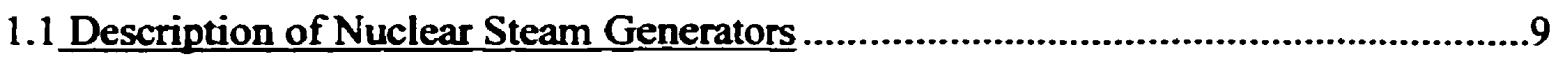

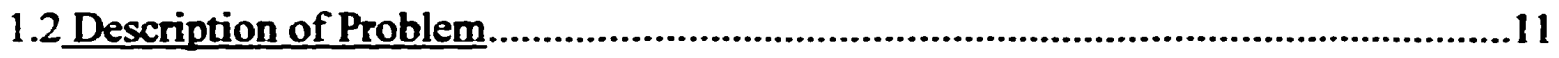

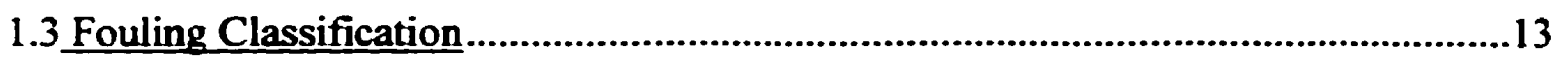

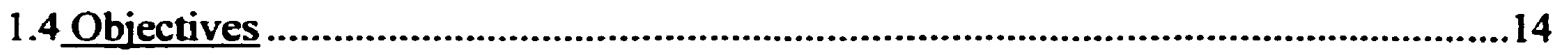

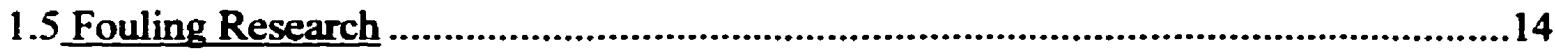

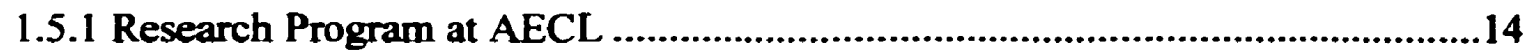

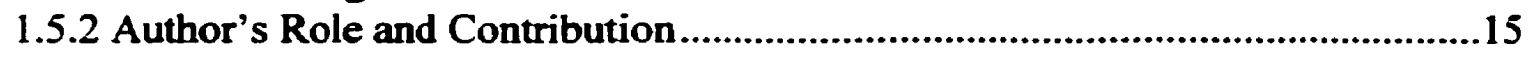

2. LITERATURE SURVEYS FOR FOULING OF SG SUPPORTS .............................27

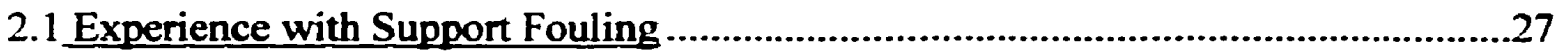

2.1 .1 Experience at Crystal River ..................................................................27

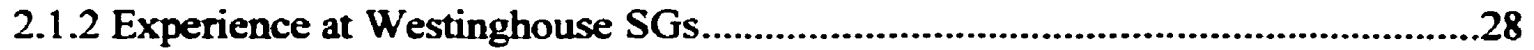

2.1.3 Experience at Bruce A................................................................................29

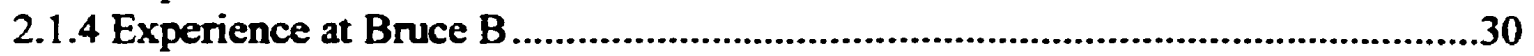

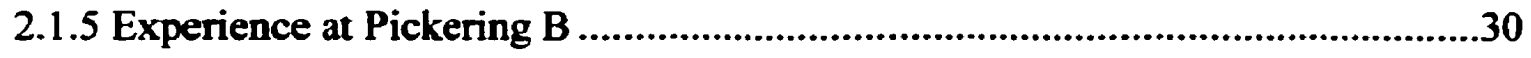

2.1.6 Experience in a Fouling Test Loop ..................................................................

2.2 Studies on Support Thermalhydraulics....................................................................

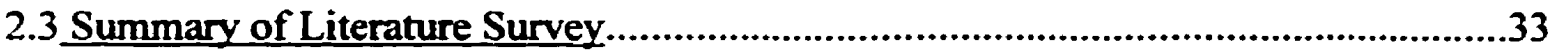

3. DEVELOPING A RESEARCH PROGRAM ......................................................41

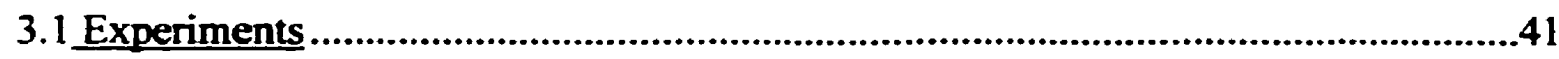

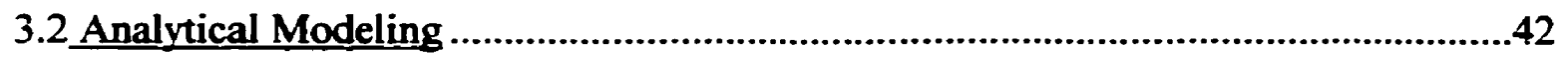

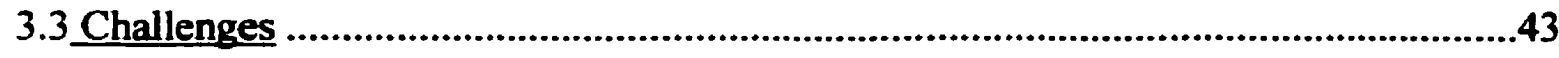




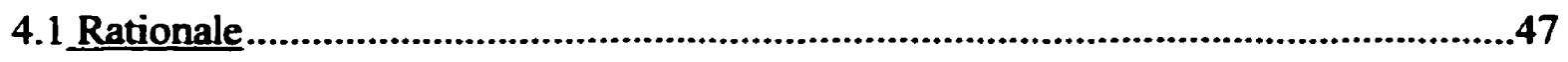

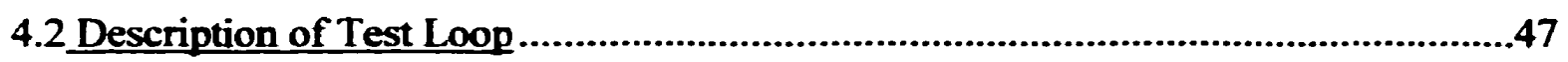

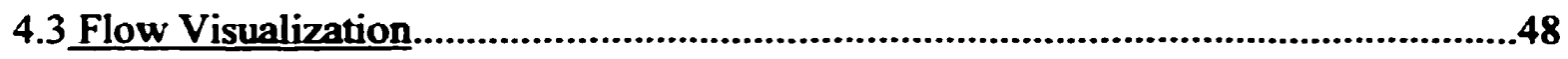

4.3.1 Results for Clean Supports .........................................................................48

4.3.2 Results for Partially-Blocked Flow Channels .................................................49

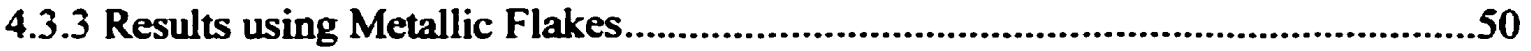

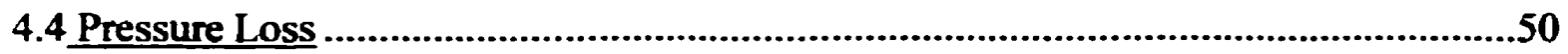

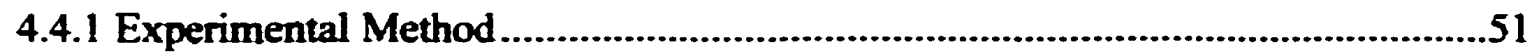

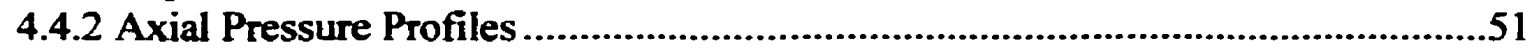

4.4.3 Calculated K-factors..........................................................................52

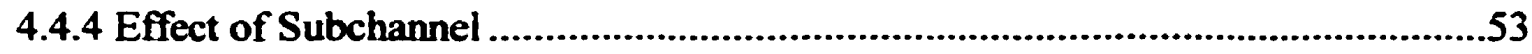

4.4.5 Effect of Edge Sharpness ...........................................................................54

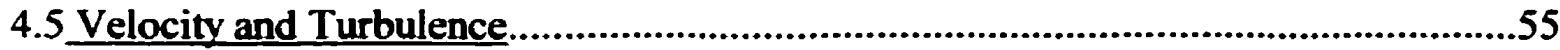

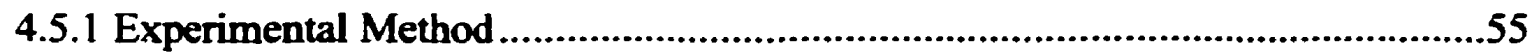

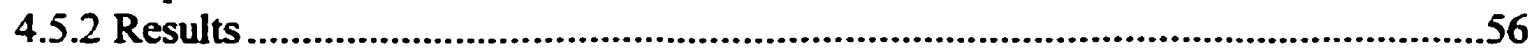

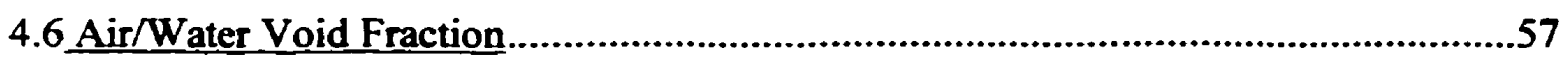

4.6.1 Experimental Method ...........................................................................57

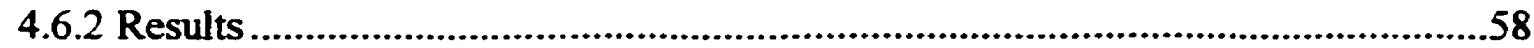

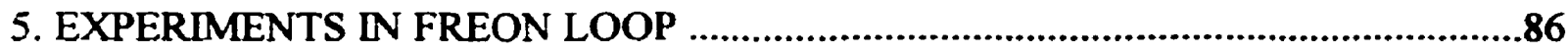

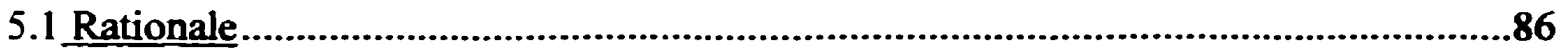

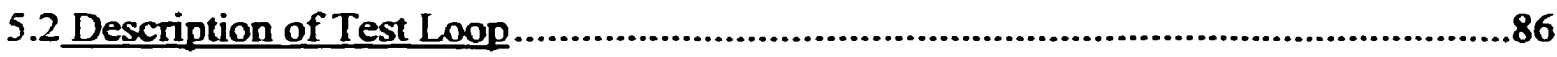

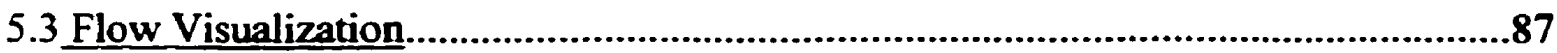

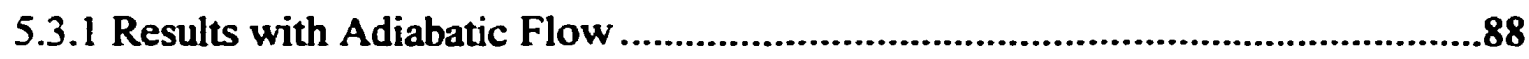

5.3.2 Results with Diabatic Flow ..................................................................89

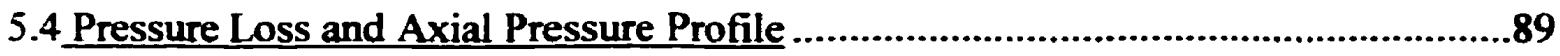

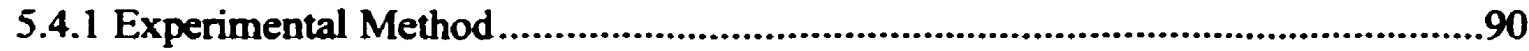

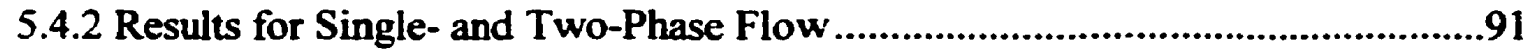

5.4.3 Comparison with Calculated Pressure Profile.............................................92

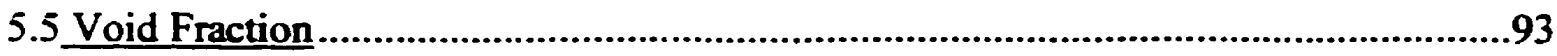

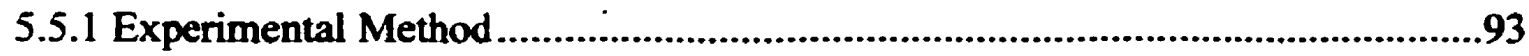

5.5.2 Results for Local Void Fraction ..................................................................94

5.5.3 Results for Axial Void Profile..............................................................96 
6. CORRELATION BETWEEN FLOW AND FOULING PATTERNS ....................131

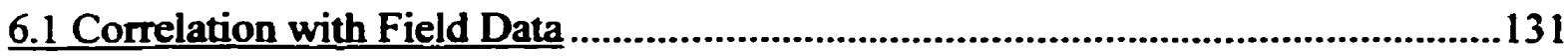

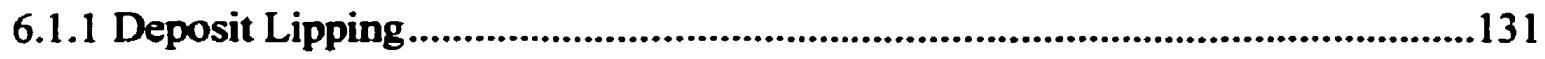

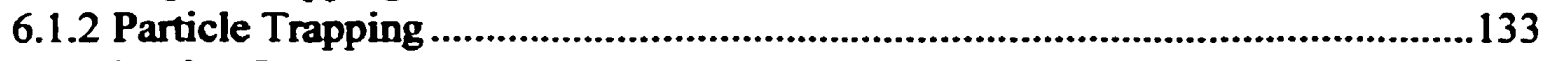

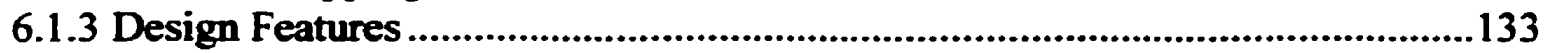

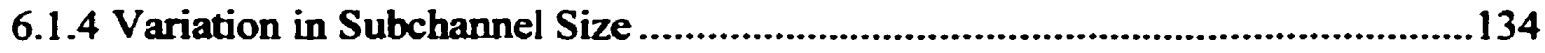

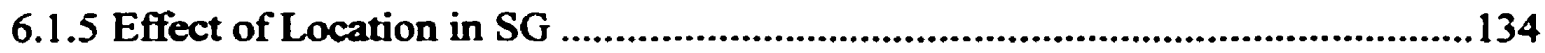

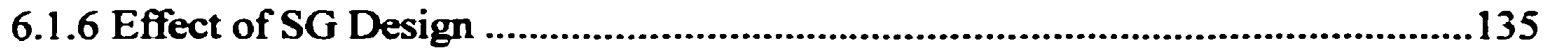

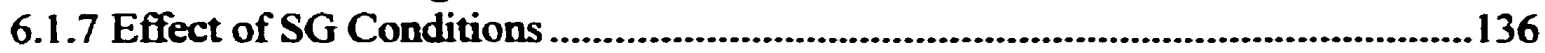

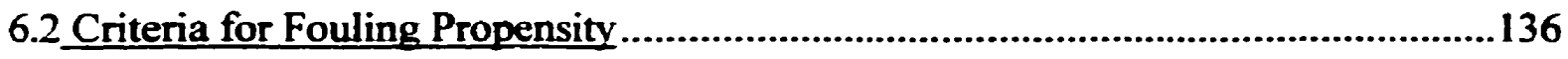

7. TSFOUL: BASIC ANAL YTICAL MODELS .........................................................

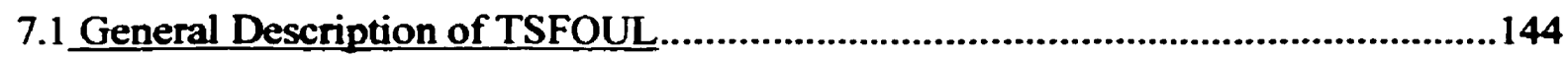

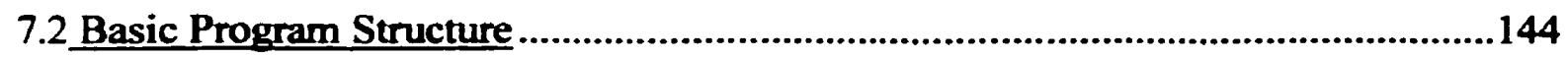

7.3 Basic Thermalhydraulic Models ......................................................................145

7.3.1 Fluid Physical Properties.....................................................................145

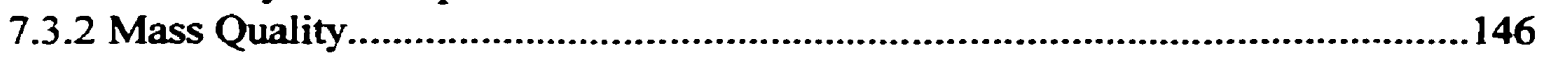

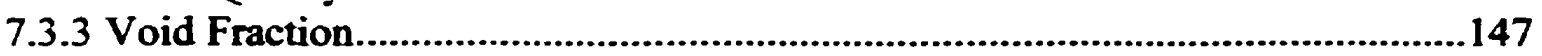

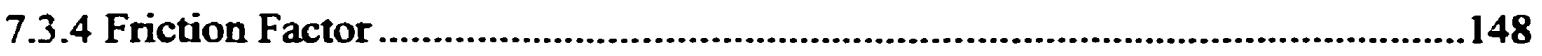

7.3.5 Two-Phase Pressure Loss .......................................................................149

7.3.6 Variation of Quality over Distance.......................................................157

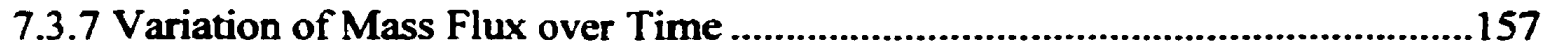

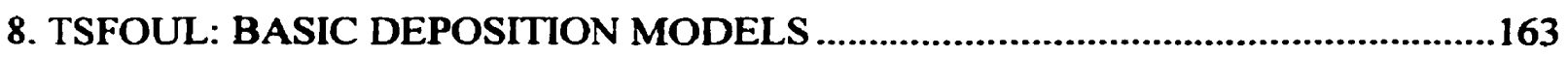

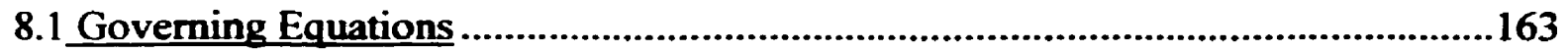

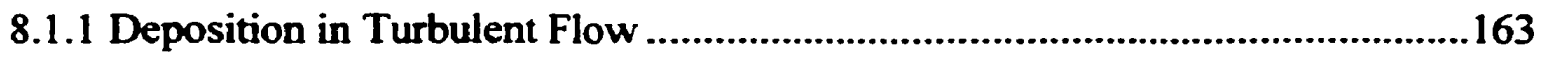

8.1 .2 Deposition in Laminar Flow.................................................................165

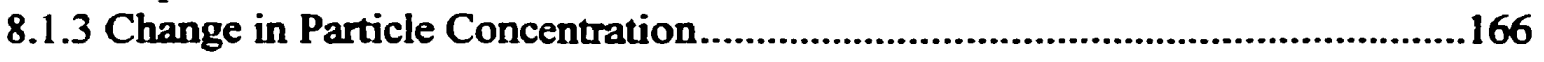

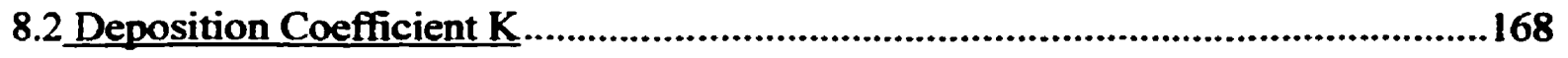

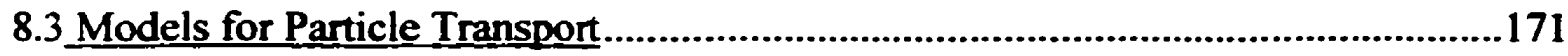

8.3.1 Deposition from Turbulent Diffusion ......................................................171

8.3.2 Deposition from Laminar Diffusion..........................................................175

8.3.3 Deposition from Inertial Coasting ........................................................179 


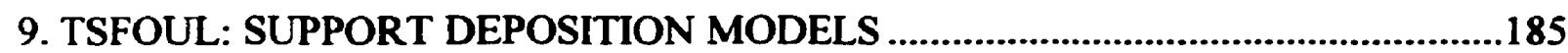

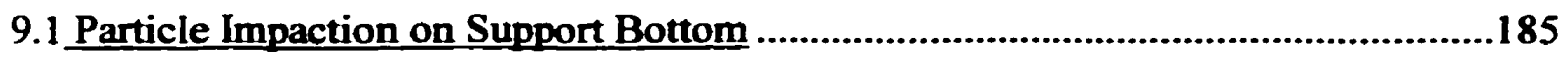

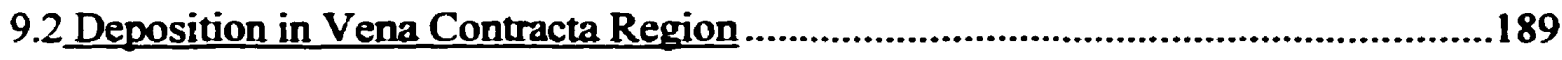

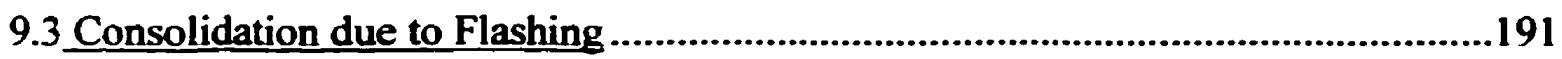

9.4 Enhanced Deposition from Flow Development on Support.................................194

9.5 Gravitational or Centrifugal Settling on Top of Support..................................195

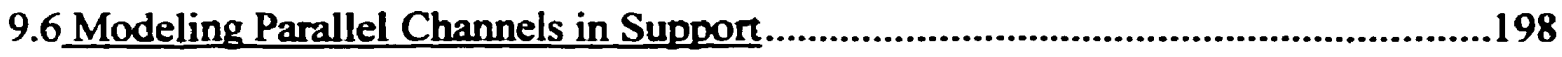

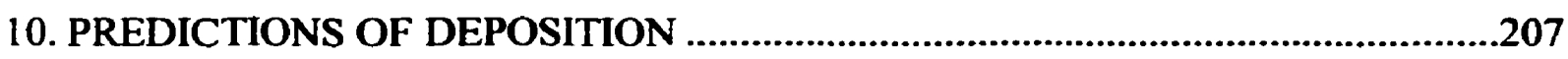

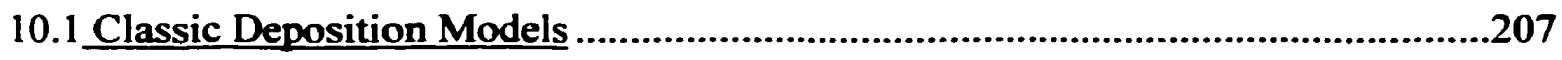

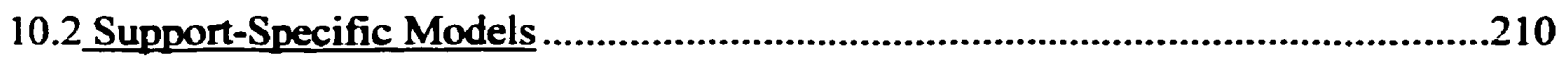

10.2.1 Effect of flow regime: turbulent vs laminar ............................................210

10.2.2 Effect of particle impaction on support bottom ............................................211

10.2.3 Effect of centrifugal and gravitational settling ..........................................212

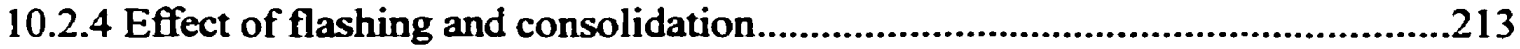

10.2.5 Effect of developing flow..................................................................214

10.2.6 Effect of unequally-sized parallel channels...........................................215

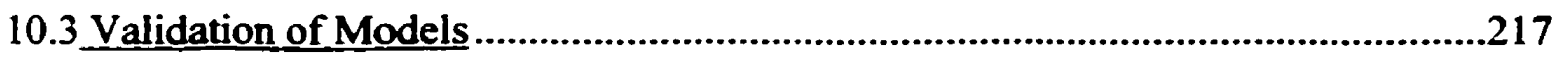

10.3.1 Comparison with Short-Term Fouling Experiments ...............................217

10.3.2 Comparison with Long-Term Fouling at Bruce A SG ...............................217

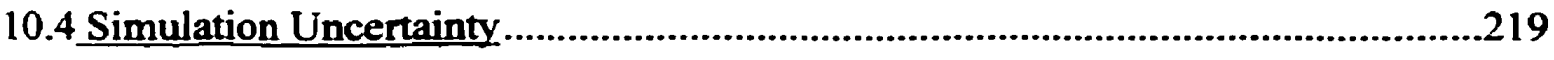

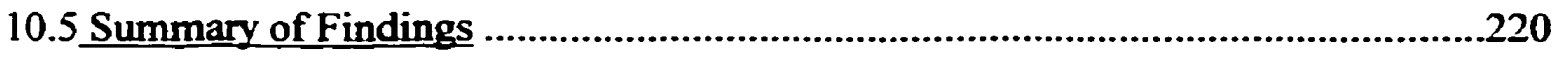

11. SUMMARY AND RECOMMENDATIONS ............................................251

11.1 Summary of Experimental Observations.......................................................251

11.2 Summary of Correlation with Fouling Patterns ...............................................252

11.3 Summary of Fouling Susceptibility Criteria .....................................................253

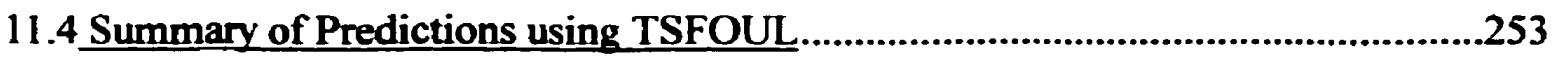




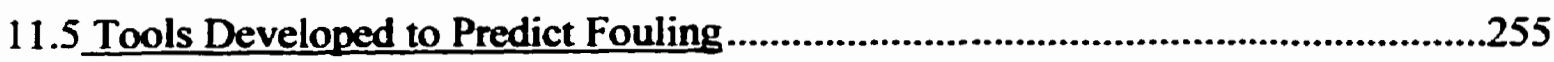

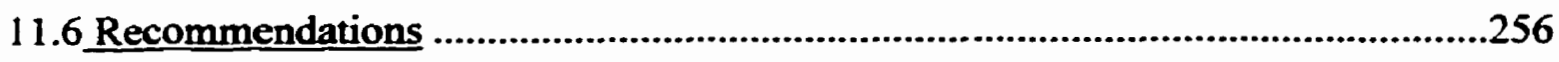




\section{List of Tables}

Table 1.1: Typical operating conditions of CANDU SGs

Table 3.1: Comparison of physical properties: SG steam/water, air/water, Freon-11 45

Table 4.1: Dimensions of mockups for air/water and comparison with actual 60

Table 4.2: Support K-factors, measured and calculated $\quad 62$

Table 4.3: Contraction, friction, and expansion components in calculated K-factors 63

Table 5.1: Dimensions of mockups for Freon and comparison with actual 98

Table 6.1: Qualitative evaluation of supports from fouling perspective 139

Table 10.1: Model options in TSFOUL $\quad 222$

Table 10.2: Reference input values in TSFOUL $\quad 223$

Table 10.3: Predicted values of K with classic deposition models 224

Table 10.4: Input values for simulation of bi-foil experiment 225 


\section{List of Illustrations}

Figure 1.1: Schematic of typical CANDU nuclear reactor system 20

Figure 1.2: Schematic of typical tube-in-shell recirculating SGs [12] 21

Figure 1.3: Bruce A steam generator \& steam drum 22

Figure 1.4: Darlington steam generator with integral preheater 23

Figure 1.5: Tube support designs (including U-bend supports) 24

Figure 2.1: Deposits on Crystal River supports [2] 36

Figure 2.2: Deposits on Bruce A Unit 2 Boiler 3 supports [5,6] 38

Figure 2.3: Deposition on experimental bifoil support $\quad 40$

Figure 4.1: Mockups tested in air/water $\quad 64$

$\begin{array}{ll}\text { Figure 4.2: Air/water test loop } & 65\end{array}$

Figure 4.3: Observed flow patterns - clean supports 66

Figure 4.4: Observed flow patterns - partially-blocked channels 68

Figure 4.5: Observed flow patterns - deposition of metallic flakes 69

$\begin{array}{ll}\text { Figure 4.6: Pressure loss apparatus } & 71\end{array}$

Figure 4.7: Pressure loss profiles for various supports $\quad 72$

Figure 4.8: Effect of subchannel on Darlington lattice bar pressure profiles 76

Figure 4.9: Effect of edge sharpness on Darlington lattice bar pressure profiles 77

$\begin{array}{ll}\text { Figure 4.10: LDV apparatus } & 78\end{array}$

Figure 4.11: Typical output from single velocity measurement 79

Figure 4.12: Velocity distribution inside sectioned broach hole $\quad 80$

$\begin{array}{ll}\text { Figure 4.13: Maps of velocity and turbulence intensity } & 81\end{array}$

$\begin{array}{ll}\text { Figure 4.14: Void apparatus } & 84\end{array}$

Figure 4.15: Map of void fraction for broached plate $\quad 85$

$\begin{array}{ll}\text { Figure 5.1: Mockups tested in Freon } & 100\end{array}$

$\begin{array}{ll}\text { Figure 5.2: Freon test loop } & 101\end{array}$ 
Figure 5.3: Schematic of Freon-11 test loop

Figure 5.4: Observed flow patterns - clean supports $\quad 103$

$\begin{array}{lr}\text { Figure 5.5: Pressure loss apparatus } & 110\end{array}$

Figure 5.6: Measured axial pressure loss profiles $\quad 111$

Figure 5.7: Measured and calculated two-phase pressure profile (for Figure 5.6b) 114

Figure 5.8: Components in overall calculated pressure profile in Figure 5.7 115

$\begin{array}{ll}\text { Figure 5.9: Void fraction apparatus } & 116\end{array}$

Figure 5.10: Maps of local void fraction - Bruce broached plate 117

Figure 5.11: Maps of local void fraction - CANDU-6 broached plate 118

Figure 5.12: Maps of local void fraction - Darlington lattice bars 122

Figure 5.13: Maps of local void fraction - Wolsong-1 formed bars 126

Figure 5.14: Measured axial void fraction profile for CANDU-6 broached plate $\quad 130$

Figure 6.1: Schematic of vena contracta region $\quad 140$

Figure 6.2: Approximate conditions on SG hot leg 141

Figure 6.3: Calculated tube-support pressure losses: $\quad 142$

Figure 6.4: Proposed support fouling mechanisms $\quad 143$

Figure 7.1: Geometry for TSFOUL 161

$\begin{array}{ll}\text { Figure 7.2: Flowchart for TSFOUL } & 162\end{array}$

Figure 9.1: Sketch of particle impaction on support bottom 201

Figure 9.2: Sketch of flashing and consolidation phenomenon 202

Figure 9.3: Suppression factor $A$ as a function of precipitate fraction 203

Figure 9.4: Sketch of enhanced deposition in developing regions 204

Figure 9.5: Sketch of settling on top of support 205

Figure 9.6: Sketch of unequally-sized channels in support 206

Figure 10.1: Classic models: reference case $\quad 226$

Figure 10.2: Sensitivity runs with classic models 228 
Figure 10.3: Effect of flow regime

Figure 10.4: Effect of particle impaction 234

Figure 10.5: Effect of settling $\quad 236$

Figure 10.6: Effect of flashing and consolidation 238

Figure 10.7: Effect of flow development 241

Figure 10.8: Effect of unequally-sized channels $\quad 242$

Figure 10.9: Prediction for bifoil fouling experiment 245

Figure 10.10: Long-term prediction for Bruce A SG 246

Figure B.1: Comparison with Idel'chik model, with varying mass flux 271

Figure B.2: Comparison with Idel'chik model, with varying flow area ratio 271 


\section{List of Appendices}

APPENDIX A: BASIC DEPOSITION MODELS

A.1 General Description of SLUDGE Code $\quad 259$

A.2 Governing Equations $\quad 259$

A.3 Deposition Coefficient K 261

A.3.1 Single-Phase Deposition Coefficient 261

A.3.2 Two-Phase Deposition Coefficient 262

A.4 Models for Particle Transport $\quad 262$

A.4.1 Thermophoresis $\quad 262$

A.4.2 Inertial coasting 263

A.4.3 Diffusion 264

A.4.4 Gravitational and Centrifugal Settling $\quad 264$

A.5 Model for Particle Attachment 265

APPENDIX B: COMPARISON OF PRESSURE LOSS MODELS 268

APPENDIX C: LISTING OF TSFOUL PROGRAM 272

APPENDDX D: TYPICAL INPUT FILE FOR TSFOUL 301

APPENDIX E: TYPICAL OUTPUT FILE FOR TSFOUL 302 


\section{Glossary}

AECL

amorphous

buffer

CANDU

cold leg

colloid

denting

derating

flashing

flow area ratio
Atomic Energy of Canada Ltd., a crown corporation

having no regular structure (opposite of crystalline)

a solution that resists change in $\mathrm{pH}$ when a small amount of acid or base is added or when the solution is diluted

CANada Deuterium Uranium (registered trademark), a nuclear power reactor developed and marketed by $A E C L *$

half-side of SG between the U-bend region at the top and the outlet of the cooled-down primary coolant

fluid suspension of particles of diameter $<0.5 \mu \mathrm{m}$

the gradual pinching or deformation of a tube due to buildup of deposits in the crevice between the tube and the support

a reduction in the station power output due to, for example, an inability to transfer all the heat generated at full power to the secondary coolant

sudden evaporation of thermally saturated liquid due to a local decrease in pressure

the free-span (tubes-only region) flow area divided by the flow area of the channels in the support

flow-assisted corrosion the flow-aided dissolution of normally protective oxide films

free span

Freon

hot leg

land region in the SG where only tubes are present; also called the approach region

trade name for fluorocarbon-based fluids commonly used as refrigerants

half-side of SG between the inlet of the hot primary coolant and the $U$-bend region at the top

portion of the support surface that is perpendicular to the flow and that contacts the SG tube, forming a corner 


\begin{abstract}
magnetite
iron oxide, $\mathrm{Fe}_{3} \mathrm{O}_{4}$, a common corrosion product found in ferrous piping systems

OTSG

once-through steam generator, in which the secondary coolant is preheated, evaporated completely, and superheated in a single pass through the SG

pitting

localized corrosion caused by impurities concentrated under deposits or in crevices

porosity in terms of the deposit: the fraction of deposit occupied by voids (e.g., a deposit with few voids will have low porosity)

porosity

in terms of the support: the inverse of the flow-area ratio (e.g., a support support with relatively small openings for flow will have low porosity)

quality (steam) the ratio of the mass of steam to the total mass of the fluid

recirculation ratio the average number of times a volume of liquid completes the circuit in the $\mathrm{SG}$ before vaporizing to steam; or, the ratio of the mass flow rate of downcomer liquid to the mass flow rate of generated steam
\end{abstract}

RSG

recirculating steam generator, in which the up-flowing secondary coolant evaporates to a relatively low-quality (up to $\sim 30 \%$ ) twophase fluid, the steam is separated from the liquid, and this liquid is returned to the inlet for further evaporation

soluble

an ion in solution, usually iron or copper in the case of SGs

stress-corrosion cracking

localized corrosion at a grain boundary, in the presence of tensile stress

thermosyphoning forced flow driven by large static head differences; velocities can be relatively high, such that the pressure losses and heat transfer behaviour resembles those of forced (pumped) convection

tritium

hydrogen atom with nucleus having two neutrons in addition to the single proton

vena contracta

position of minimum flow area downstream of a sudden contraction in flow area, which causes a separation of the boundary layer from the wall 
void fraction

wastage

water-lancing the fraction of volume occupied by the gas or vapour phase in a two-phase fluid

severe case of pitting* due to, for example, phosphoric acid concentrated under deposits when sodium phosphate is added to the water for $\mathrm{pH}$ control and buffering*

a technique for cutting hard deposits using a jet of water under very high pressures 


\section{Nomenclature}

\begin{tabular}{|c|c|c|}
\hline a & $\mathbf{m}$ & half-length of rectangular channel \\
\hline A & - & deposit removal or suppression factor \\
\hline A & $\mathrm{m}^{2}$ & flow area \\
\hline$A_{0}$ & - & value of suppression factor $A$ assuming no consolidation \\
\hline b & $\mathbf{m}$ & half-width of rectangular channel \\
\hline B & $\mathbf{m} / \mathbf{s}$ & boiling coefficient \\
\hline $\mathrm{C}$ & - & impaction coefficient \\
\hline $\mathrm{C}_{\mathrm{c}}$ & - & effective fraction of open flow area in vena contracta region \\
\hline $\mathrm{C}_{\mathrm{o}}$ & - & void distribution parameter in drift flux equation (Eq. 7.4) \\
\hline $\mathrm{Cp}$ & J/kg.K & specific heat \\
\hline $\mathrm{d}_{\mathrm{b}}$ & m & bubble diameter \\
\hline D & $\mathrm{m}^{2} / \mathrm{s}$ & diffusivity \\
\hline $\mathrm{D}_{\mathrm{hy}}$ & $\mathbf{m}$ & channel hydraulic diameter (= 4A/S, where $S$ is wetted perimeter) \\
\hline $\mathrm{D}_{\mathrm{m}}$ & m & mean diameter of annular flow channel \\
\hline $\mathrm{D}_{\mathrm{s}}$ & m & particle diameter \\
\hline $\mathrm{D}_{\text {tube }}$ & m & tube diameter \\
\hline $\mathrm{E}_{\mathbf{a}}$ & $\mathrm{J} / \mathrm{mol}$ & activation energy \\
\hline $\mathbf{F}$ & $\mathrm{kg} / \mathrm{kg}$ & fraction of precipitate in deposit \\
\hline$f$ & - & Darcy friction factor \\
\hline g & $\mathrm{m} / \mathrm{s}^{2}$ & gravitational acceleration $(=9.81)$ \\
\hline G & $\mathrm{kg} / \mathrm{m}^{2} . \mathrm{s}$ & mass flux \\
\hline h & $\mathrm{J} / \mathrm{kg}$ & specific enthalpy \\
\hline h & $\mathrm{J} / \mathrm{s} . \mathrm{m}^{2}$ & wall heat transfer coefficient \\
\hline $\mathrm{h}_{\mathrm{fg}}$ & $\mathrm{J} / \mathbf{k g}$ & latent heat of vaporization \\
\hline j & $\mathrm{m} / \mathrm{s}$ & superficial velocity \\
\hline $\mathbf{k}$ & J/s.m.K & thermal conductivity \\
\hline k & $\mathbf{J} / \mathbf{K}$ & Boltzmann constant $\left(=1.3807 \times 10^{-23}\right)$ \\
\hline K & - & flow resistance coefficient \\
\hline
\end{tabular}




\begin{tabular}{|c|c|c|}
\hline $\mathbf{K}$ & $\mathrm{m} / \mathrm{s}$ & deposition coefficient \\
\hline $\mathrm{K}_{\mathrm{o}}$ & $\mathrm{m} / \mathrm{s}$ & attachment constant in correlation for $\mathbf{K}_{\mathrm{A}}$ \\
\hline $\mathbf{k}_{\mathrm{c}}$ & $\mathrm{m}^{-1}$ & inverse of streamline curvature radius \\
\hline $\mathrm{k}_{\mathrm{s}}$ & J/s.m.K & deposit thermal conductivity \\
\hline KE & $\mathbf{J}$ & kinetic energy \\
\hline $\mathrm{L}$ & m & length \\
\hline $\mathrm{L}$ & m & mean free path of fluid molecule $(=2 \mu / \rho \mathrm{\rho u})$ \\
\hline m & $\mathrm{kg} / \mathrm{s}$ & mass flow rate \\
\hline m & $\mathrm{kg} / \mathrm{m}^{2}$ & mass of particles deposited per unit area (Appendix A) \\
\hline M & $s^{-1}$ & slope of velocity distribution (Eq. 8.41) \\
\hline $\mathrm{N}$ & $\mathrm{mol}^{-1}$ & Avogadro's number $\left(=6.022 \times 10^{-23}\right)$ \\
\hline $\mathrm{N}$ & - & fraction of particles \\
\hline $\mathrm{Nu}$ & - & Nusselt number $\left(=h D_{h y} / k\right)$ \\
\hline$P$ & MPa & pressure \\
\hline$P_{\text {sat }}$ & MPa & saturation pressure \\
\hline $\mathrm{Pe}$ & - & Peclet number $(=\operatorname{Re} \mathrm{Pr})$ \\
\hline $\operatorname{Pr}$ & - & Prandtl number $(=\mathrm{Cp} \mu / \mathrm{k})$ \\
\hline Q & $\mathrm{J} / \mathrm{s} \cdot \mathrm{m}^{2}$ & heat flux \\
\hline $\mathrm{R}$ & $\mathrm{J} / \mathrm{K} \cdot \mathbf{m o l}$ & molar gas constant $(=8.314)$ \\
\hline $\operatorname{Re}$ & - & Reynolds number $\left(=\rho u D_{h y} / \mu\right)$ \\
\hline $\mathbf{S}$ & $\mathrm{kg} / \mathrm{s}$ & rate of particulate flow, rate of precipitation \\
\hline Sc & - & Schmidt number $(=v / D)$ \\
\hline $\mathrm{Sh}$ & - & Sherwood number \\
\hline $\mathrm{t}$ & $\mathbf{s}$ & elapsed time \\
\hline$T_{b}$ & ${ }^{\circ} \mathrm{C}$ & bulk fluid temperature \\
\hline$t_{p}^{+}$ & - & non-dimensional particle relaxation time \\
\hline $\mathrm{T}_{\text {sat }}$ & ${ }^{\circ} \mathrm{C}$ & saturation temperature \\
\hline$T_{w}$ & ${ }^{\circ} \mathrm{C}$ or $\mathrm{K}$ & wall temperature \\
\hline $\mathbf{u}$ & $\mathrm{m} / \mathrm{s}$ & actual velocity \\
\hline $\mathrm{u}^{*}$ & $\mathbf{m} / \mathbf{s}$ & friction velocity \\
\hline
\end{tabular}




\begin{tabular}{|c|c|c|}
\hline $\mathrm{V}_{\mathrm{gj}}$ & $\mathbf{m} / \mathbf{s}$ & weighted mean drift velocity \\
\hline $\mathbf{w}$ & $\mathbf{m}$ & width of annular flow channel \\
\hline $\mathbf{w}_{\mathrm{jet}}$ & $\mathbf{m}$ & width of oncoming fluid jet \\
\hline X & - & parameter in flow development $\left(=z / D_{h y}\right)$ \\
\hline$z$ & m & axial distance \\
\hline$\alpha$ & $\mathrm{m}^{3} / \mathrm{m}^{3}$ & void fraction \\
\hline$\beta_{\mathrm{s}}$ & - & deposit porosity \\
\hline$\beta_{\text {sup }}$ & - & support porosity (= support flow area / free-span flow area) \\
\hline$\chi_{\mathrm{ch}}$ & $\mathrm{kg} / \mathrm{kg}$ & thermodynamic (equilibrium) quality \\
\hline$\chi$ & $\mathrm{kg} / \mathbf{k g}$ & mass quality \\
\hline$\delta$ & $\mathbf{m}$ & deposit thickness \\
\hline$\delta \mathbf{z}$ & m & incremental distance interval \\
\hline$\Delta \mathrm{P}$ & $\mathrm{kg} / \mathrm{m} \cdot \mathrm{s}^{2}, \mathrm{~Pa}$ & pressure difference (loss) \\
\hline$\Delta \mathrm{T}_{\text {sub }}$ & ${ }^{\circ} \mathrm{C}$ & subcooling $\left(=T_{\text {sat }}-T_{b}\right)$ \\
\hline$\Delta z$ & $\mathrm{~m}$ & distance interval \\
\hline$\Delta \phi$ & $\mathrm{kg} / \mathrm{kg}$ & change in particle concentration \\
\hline$\varepsilon$ & $\mathbf{m}$ & surface roughness \\
\hline$\phi$ & $\mathrm{kg} / \mathrm{kg}$ & particle concentration \\
\hline$\Phi^{2}$ & - & two-phase multiplier ( $=1$ for single phase) \\
\hline$\lambda$ & $s^{-1}$ & re-entrainment term \\
\hline$\mu$ & $\mathrm{kg} / \mathrm{m} . \mathrm{s}$ & dynamic viscosity \\
\hline$v$ & $\mathrm{~m}^{2} / \mathrm{s}$ & kinematic viscosity $(=\mu / \rho)$ \\
\hline$\rho$ & $\mathrm{kg} / \mathrm{m}^{3}$ & density \\
\hline$\rho_{\mathrm{s}}$ & $\mathrm{kg} / \mathrm{m}^{3}$ & particle density \\
\hline$\sigma$ & $\mathrm{kg} / \mathrm{s}^{2}$ & surface tension \\
\hline$\tau$ & - & Idel'chik flow development factor \\
\hline$\tau_{\mathrm{w}}$ & $\mathrm{kg} / \mathrm{m} \cdot \mathrm{s}^{2}$ & wall shear stress $\left(=f \rho u^{2} / 8\right)$ \\
\hline$\Psi$ & - & non-dimensional inertia parameter \\
\hline
\end{tabular}


subscripts

I申 one-phase

$2 \phi \quad$ two-phase

A attachment (of particle to surface)

acc acceleration

att attachment (of boundary layer to wall)

area area change

ave average

b bubble

b bulk

B boiling

bd bubble detachment

c cementing agent

C centrifugal settling

calc calculated

con sudden contraction

D diffusion

dep deposition

evap evaporation

exp sudden expansion

expt experimental

$\mathrm{f} \quad$ liquid

F filtering, impaction

fldev flow development

fric friction

fs free span

fu liquid, in a separated flow

g gas or vapour

G gravitational settling 


\begin{tabular}{ll} 
gr & gravitation \\
hy & hydraulic \\
i & current node \\
i-l & previous node \\
imp & impaction \\
I & inertial coasting \\
L, lam & laminar \\
loc & local \\
min & minimum \\
prev & previous \\
S & solid (particle) \\
sat & saturation \\
st & sticking \\
sub & subcooled \\
sup & support \\
T, turb & turbulent \\
T & transport \\
th & thermodynamic \\
TH & thermophoresis \\
tot & total \\
V, vena & vena contracta region \\
w & wall \\
z & distance \\
incremental distance interval \\
\hline
\end{tabular}




\section{Chapter 1}

\section{INTRODUCTION}

\subsection{Description of Nuclear Steam Generators}

Nuclear power stations currently in use rely on the fissioning of uranium-235 and similar heavy atoms to produce heat. As shown in Figure 1.1, the heat produced in the nuclear reactor is transferred to a coolant. In $\mathrm{CANDU}^{* 1}$ reactors, the coolant is pressurized heavy water $\left(D_{2} O\right)$ which also acts to moderate the fission chain reaction. The heat is transported via this primary coolant to boilers or steam generators (SGs), which then transfer the heat to a light water $\left(\mathrm{H}_{2} \mathrm{O}\right)$ secondary coolant operating at high temperature $\left(\sim 250^{\circ} \mathrm{C}\right)$ and high pressure $(-4.5 \mathrm{MPa})$. This secondary coolant vaporizes to steam which then drives a series of turbines for electricity production. The steam is subsequently condensed and returned to the SG as feedwater.

Steam generators for nuclear power stations are typically of the vertical tube-in-shell type, shown in Figure 1.2 in the form of a recirculating SG or RSG*. The primary coolant from the nuclear reactor is pumped at high pressure $(\sim 10 \mathrm{MPa})$ through hundreds of 1 to $2 \mathrm{~cm}$ diameter U-tubes, which are enclosed within a vessel containing the secondary coolant. These tubes are fixed to a tube sheet at the bottom of the SG, which separates the primary $\mathrm{D}_{2} \mathrm{O}$ coolant from the secondary $\mathrm{H}_{2} \mathrm{O}$ coolant. Typical operating conditions for two different CANDU SGs are listed in Table 1.1.

Some SGs are of the once-through type, called OTSG*, in which the secondary coolant is pumped past the hot tubes and becomes superheated steam at the outlet. CANDU SGs, such as those at the Bruce $A$ or Darlington stations (Figures 1.3 and 1.4), are of the recirculating type, in which the secondary coolant rises by natural convection past the hot

1 Terms designated with an asterisk are explained in the GLOSSARY section. 
tubes. While the steam moves on to the turbines, the water and any excess liquid shed by the cyclone separators (located at the top of the SG) returns to the bottom of the SG via an annular downcomer. Because of the density difference between the steam-water mixture and the downcomer liquid, natural circulation (or, more precisely, thermosyphoning*) is established in the circuit thereby ensuring heat removal from the heated surfaces.

The downcomer liquid combines with feedwater which makes up for the loss of inventory from steam generation. After the feedwater has been heated to an optimum temperature using extraction feedwater heaters (not shown), it is typically preheated internally using baffle plates (Figures 1.2 and 1.4). The Bruce A SG is unique in that it uses an external preheater (Item 13 in Figure 1.3) instead of the internal baffle plates.

For nuclear reactors in particular, the SGs are designed to be exceptionally robust. From the standpoint of cost, the stations cannot afford tube leaks and hence loss of expensive heavy water from the primary side. From the standpoint of safety, large leaks from, for example, SG tube ruptures could put the nuclear fuel at risk of overheating and releasing radioactive fission products. Also, as the primary coolant transports tritium* and other radioactive by-products, SG maintenance leads to radiation doses to the workers and must therefore be minimized.

For robustness, the tubes are sealed to the tube sheet at the inlet and outlet ends, and are separated by plates or grids of tube supports within the main body of the SG. As shown in Figure 1.2, the supports are typically positioned at 1-meter elevations, as well as at various angles in the top U-bend region of the tube bundle. They prevent the tubes from touching each other and prevent excessive flow-induced vibration in the boiling flow.

Figure 1.5 shows designs of supports used in various CANDU and other SGs. Simple plates such as the drilled-hole plates or broached plates (either trefoil or quatrefoil) are the most common. The earliest CANDU SGs used broached plates, and these now appear 
prone to fouling, as this thesis will demonstrate. Grids consisting of flat bars such as the lattice bars, first considered too fragile, are becoming more common because they are less flow-restrictive, they support the tube over a greater height, and they seem less prone to fouling. The formed bars have features related to both these types.

There are five support designs currently in use in CANDUs. [Experimental mockups of these supports are shown in Figures 4.1 and 5.1.] They are, in roughly chronological order:

1. Bruce-type broached plates, in use at the Bruce A, Bruce B, and Pickering B stations (all in Ontario);

2. Pickering-type lattice bars, in use at Pickering A (Ontario);

3. CANDU-6-type broached plates, in use at Embalse (Argentina), Cernavoda (Romania), Pt. Lepreau (New Brunswick), and Gentilly-2 (Quebec);

4. Darlington-type lattice bars, in use at all Darlington stations (Ontario), at Wolsung-2 to -4 (South Korea), and at the two future Qinshan stations (China);

5. Wolsung-1-type formed bars, in use at Wolsung-1 (South Korea).

The evolution of supports - from broached plates to lattice bars and to formed bars-has been largely one of trial and error. The work presented in this thesis has endeavored to bring about greater understanding of the impact of support design, and a better basis for support selection.

\subsection{Description of Problem}

Nuclear SGs, in comparison to boilers in other industrial applications, perform extremely well. For example, by 1990, the overall failure rate for tubes in CANDU SGs was 0.003 $\%$ for the population of over a half million tubes. The total number of tubes removed 
from service by plugging was 74, and about a third of these were plugged following removal for metallurgical examination [1].

To achieve such performance, operators of nuclear SGs world-wide have addressed a wide variety of corrosion and mechanically-induced problems $[2,3,4]$. Tube wastage* and thinning were among the first corrosion problems; after these were resolved, denting* ${ }^{*}$ stress-corrosion cracking*, tube pitting*, and fretting wear dominated.

A relative newcomer to this group is fouling and blockage of the supports that separate and restrain the SG tubes. Support fouling does not affect the integrity of the SG tubes, unless it provides an opportunity for corrosion of the tube within the tube-to-support crevice. In recirculating SGs, the trouble lies with potential secondary-side flow oscillations caused by large flow resistances by blocked flow holes, especially in the upper high-quality* regions of the SG.

In October of 1986, when an oscillation was observed in the level of one of the Bruce A Nuclear Generating Station (BNGS-A) steam drums, it was not immediately associated with fouling. [The Bruce A SG, shown in Figure 1.3, is unusual in its use of steam drums common to a set of SGs, instead of steam-separation components within each SG.] A computer analysis revealed that the oscillation was most likely caused by flow blockage in the upper part of the SG. Large flow resistances in regions of high quality and the accompanying low flow rates are known to render a thermosyphoning system unstable. The amplitude of the oscillation became severe enough to force derating* of the unit in 1988 March. During a shutdown later that year, the upper two broached support plates were inspected through holes drilled in the shell, and were found to be about $80 \%$ blocked. The support plates were partially cleaned using a water-lancing* technique, and the unit was put back in service.

The blockage was caused by the long-term buildup of primarily magnetite*, which is a product of flow-assisted corrosion*. The fact that fouling was the limiting factor in the 
SG operation was unexpected: fretting from flow-induced vibration was a greater concern, and the support plates had been designed to promote stiffness. Also, the design was thought to discourage deposition, because flow would accelerate through the broach holes and therefore scour them.

Water chemistry and operating conditions are known to influence fouling. There is now strong suspicion that flow patterns play a decisive role, and that these patterns are controlled in part by the geometry of SG components.

\subsection{Fouling Classification}

The topic of fouling in heat exchangers has been studied extensively, owing to the enormous industrial costs associated with inefficient heat transfer. The classification of fouling on heated surfaces is based on the liquid-solid interface, and has five primary categories [5]:

1. crvstallization fouling, either by precipitation from solution of dissolved substances onto the heated surface (also known as scaling), or by solidification of a pure substance from a melt;

2. particulate fouling; which is an accumulation of suspended solids onto the heated surface;

3. chemical-reaction fouling, in which reaction products deposit at the heated surface (but the surface material itself is not a reactant);

4. corrosion fouling, which is an accumulation of indigenous corrosion products on the heated surface;

5. biological fouling, which involves atlachment of macro-organisms and/or microorganisms along with their adherent slimes.

For all these categories, fouling typically progresses along the following stages: 
(1) initiation or surface conditioning, (2) transport of fouling agents to the surface, (3) attachment or adhesion to the surface, (4) removal, in the form of re-entrainment, erosion, or spalling, and (5) ageing (e.g., consolidation).

The fouling of supports and other surfaces inside the SG is primarily one of precipitate and particulate fouling (Categories 1 and 2) [6], with the fluid entraining a mixture of solubles*, colloidal* substances, and particulates.

\subsection{Objectives}

The design of some supports is believed to actively contribute to blockage problems by creating a thermalhydraulic environment favourable to deposition. The goal of this work was therefore to better understand the role that thermalhydraulics plays in the fouling of SGs, and of supports in particular. From a practical standpoint, this amounts to assessing the fouling propensity of different support designs, and to recommending methods of predicting and mitigating support fouling.

These are not straightforward tasks. Thermalhydraulic influences are not readily separated from other factors such as water chemistry, SG design, and operating history. Fouling information from the field required for validation of prediction models is scarce and often anecdotal. High-pressure, high-temperature fouling experiments in the lab are expensive and time-consuming.

\subsection{Fouling Research}

\subsubsection{Research Program at AECL}

The fouling problems at the Bruce A NGS forced the Canadian nuclear industry to seriously consider the issue of SG fouling. The Bruce incident is not an isolated one, 
considering that blockage has now been detected at the Point Lepreau and the Pickering B stations, both of which use broached support plates similar to those at the Bruce $A$ station. The radiation hazards and economic penalties associated with wholesale SG replacement are, of course, not trivial. Faced with the dilemma of rejuvenation versus replacement, the CANDU Owners' Group (COG), composed of AECL* and utilities that own CANDU reactors, has increased funding in SG research in recent years.

Among the COG contracts undertaken at AECL's Chalk River Laboratories (CRL), one involves the study of tube and support fouling. Started in 1991, this project encompasses the following:

- experimental and theoretical studies of the effect of water chemistry on deposition rates (C. Tumer),

- numerical modeling of the distribution of deposits among SG components (Y. Liner and C. Turner),

- numerical modeling of flow patterns near supports (A. Banas), and

- an experimental study of flow patterns near supports (H. Rummens).

\subsubsection{Author's Role and Contribution}

As several researchers have been involved in this project, it is important to outline precisely what topics covered in this thesis have been contributed by the author herself:

\section{Work undertaken for the COG project:}

- Experimental studies of flow patterns near supports were planned, carried out, and analyzed by the author. Guidance was provided by fellow researchers $C$. Turner and G. Dimmick, both at CRL, and by thesis supervisor Prof. J.T. Rogers at Carleton University in Ottawa. 
- The work included hiring and guiding undergraduate students, and supervising technical staff. Students and technologists assisted with measurements. Test-sections were fabricated by CRL machine-shop staff. For reasons of accountability and safety, the Freon-1 1 * flow loop could only be operated by technologists trained and licensed in the handling of refrigerants.

- The author was responsible for making decisions regarding research direction, for preparing yearly proposals, and for writing quarterly progress reports as required by COG. The work was documented formally by the author in five COG reports.

\section{Work undertaken beyond the COG project:}

- Analytical modeling (i.e., the development of the TSFOUL program described in Chapters 7 to 9) was not part of the COG project and was therefore carried out by the author during off-duty hours. Guidance was provided mostly by the thesis supervisor, Prof. J.T. Rogers.

- The author presented the work at two conferences [7,8]; a journal paper is in preparation [9].

The next Chapter summarizes reports from the field regarding SG support fouling, and literature papers on the thermalhydraulic environment near SG supports.

\section{References}

[1] R. Dyck, P. Spekkens, A. Marchand and K. Verma, “Operational Experience with Steam Generators in Canadian Nuclear Power Stations", Proceedings of the $1^{\text {st }}$ Steam Generator and Heat Exchanger Conference (sponsored by the Canadian Nuclear Society), 1990 April 30 to May 2, Toronto, Canada, p. I-10. 
[2] J.C. Blomgren, "Steam Generator Performance Worldwide", Proceedings of the $1^{\text {st }}$ Steam Generator and Heat Exchanger Conference (sponsored by the Canadian Nuclear Society), 1990 April 30 to May 2, Toronto, Canada, p. I-1.

[3] O.S. Tatone, R.L. Tapping and L. Stipan, "Steam Generator Tube Performance: Experience with Water-Cooled Nuclear Power Reactors during 1986", AECL Report No. AECL-10000, 1992 March.

[4] S.J. Green, "Thermal, Hydraulic, and Corrosion Aspects of PWR Steam Generator Problems", Heat Transfer Engineering, Vol.9, No.1, pp. 19-67 (1988).

[5] N. Epstein, "Thinking about Heat Transfer Fouling: A 5 x 5 Matrix", Heat Transfer Engineering, Vol.4, No.1, pp. 43-56 (1983).

[6] M.W. Rootham, "Sludge versus Scale: Where is the Truth?", Proceedings of the Steam Generator Sludge Management Workshop, Myrtle Beach, South Carolina, 1996 August 19-21, also as Report No. EPRI TR-108047, 1997 July, p.B1-1.

[7] H.E.C. Rummens and C.W. Turner, "Experimental Study of Flow Patterns near Tube Support Structures", Proceedings of the $2^{\text {nd }}$ International Steam Generator and Heat Exchanger Conference, 1994 June 13-15, Toronto, Canada.

[8] H.E.C. Rummens, C.W. Turner, and J.T. Rogers, "The Effect of Tube-Support Design on Steam Generator Fouling Susceptibility", Proceedings of an Engineering Foundation Meeting titled "Understanding Heat Exchanger Fouling and Mitigation", 1997 May 11 -16, Lucca, Italy.

[9] H.E.C. Rummens, J.T. Rogers, and C.W. Turner, "The Thermalhydraulics of Tube-Support Fouling in Nuclear Steam Generators", in preparation, to be submitted to Nuclear Technology. 
[10] J.M. Pietralik and A.O. Campagna, "Bruce B Steam Generator U-Bend Fretting: Thermalhydraulic Analysis", protected Ontario Hydro Report No. CI-FLOW-61, 1990 July.

[11] J.M. Pietralik, "Thermalhydraulic Analysis of the CANDU 6 Point Lepreau NGS Steam Generators", protected COG Report No. COG-94-18, 1994 April.

[12] M. Yetisir, private communication, 1998 December. 
Table 1.1: Typical operating conditions of CANDU SGs

\begin{tabular}{|l|c|c|}
\hline & Bruce B SG [1,10] & $\begin{array}{c}\text { Pt Lepreau } \\
\text { (CANDU-6) SG } \\
{[1,11]}\end{array}$ \\
\hline Primary flow rate [kg/s] & 1420 & 1890 \\
\hline Primary inlet temp [ ${ }^{\circ}$ C] & 304 & 310 \\
\hline Primary inlet quality [\%] & 0 & 4.4 \\
\hline Primary pressure [MPa] & 9.3 & 9.9 \\
\hline Feedwater flow rate [kg/s] & 170 & 260 \\
\hline Secondary inlet temp [ $\left.{ }^{\circ} \mathrm{C}\right]$ & 246 & 187 \\
\hline Secondary ave quality range [\%] & 0 to 16 & 0 to 16 \\
\hline Secondary pressure [MPa] & 4.4 & 4.7 \\
\hline Secondary recirculation ratio* & $\sim 5.2$ & $\sim 5.1$ \\
\hline Secondary flow velocity [m/s] & 0.5 to 6 & 0.5 to 5 \\
\hline Overall heat transfer rate $[\mathrm{MW}]$ & 290 & 520 \\
\hline Average heat flux $\left[\mathrm{kW} / \mathrm{m}^{2}\right]$ & 125 & 164 \\
\hline Number of tubes & 4195 & 3542 \\
\hline Tube outer diameter [m] & 0.013 & 0.0159 \\
\hline Tube inner diameter [m] & 0.0108 & 0.0137 \\
\hline Tube pitch [m] & 0.0203 triangular & 0.0241 triangular \\
\hline Tube material & Inconel 600 & Incoloy 800 \\
\hline Shroud inside radius [m] & 1.045 & 1.156 \\
\hline Number of horizontal supports & 7 & 9 \\
\hline Horiz. support type \& material & trefoil broached plate & trefoil broached plate \\
\hline Horiz. support material & carbon steel & 410 stainless steel \\
\hline Horiz. support porosity* & 0.42 & 0.61 \\
\hline Number of supports in U-bend & 3 & 7 \\
\hline Internal preheater & No & Yes \\
\hline
\end{tabular}


Figure 1.1: Schematic of typical CANDU nuclear reactor system

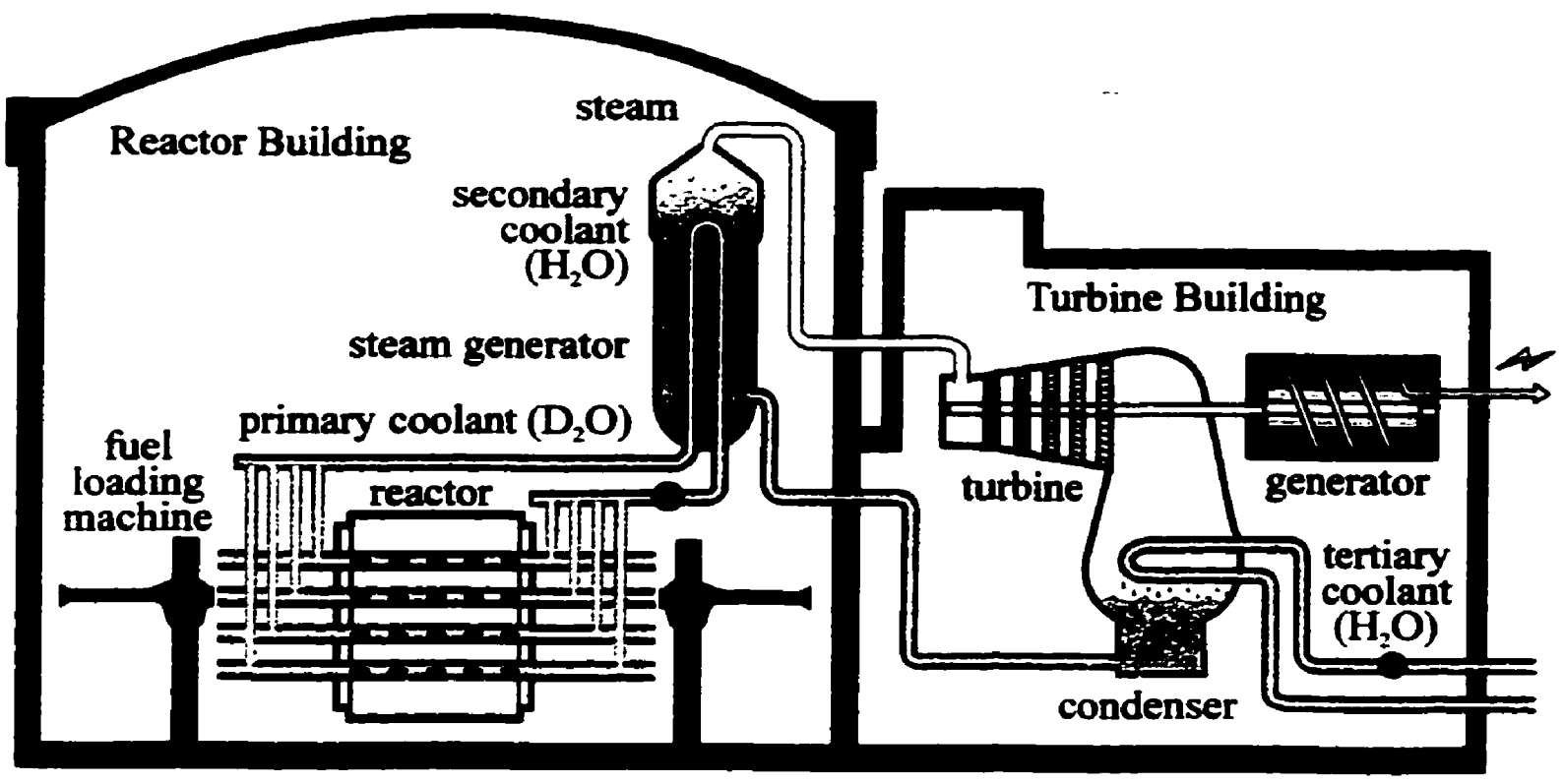


Figure 1.2: Schematic of typical tube-in-shell recirculating SGs [12]

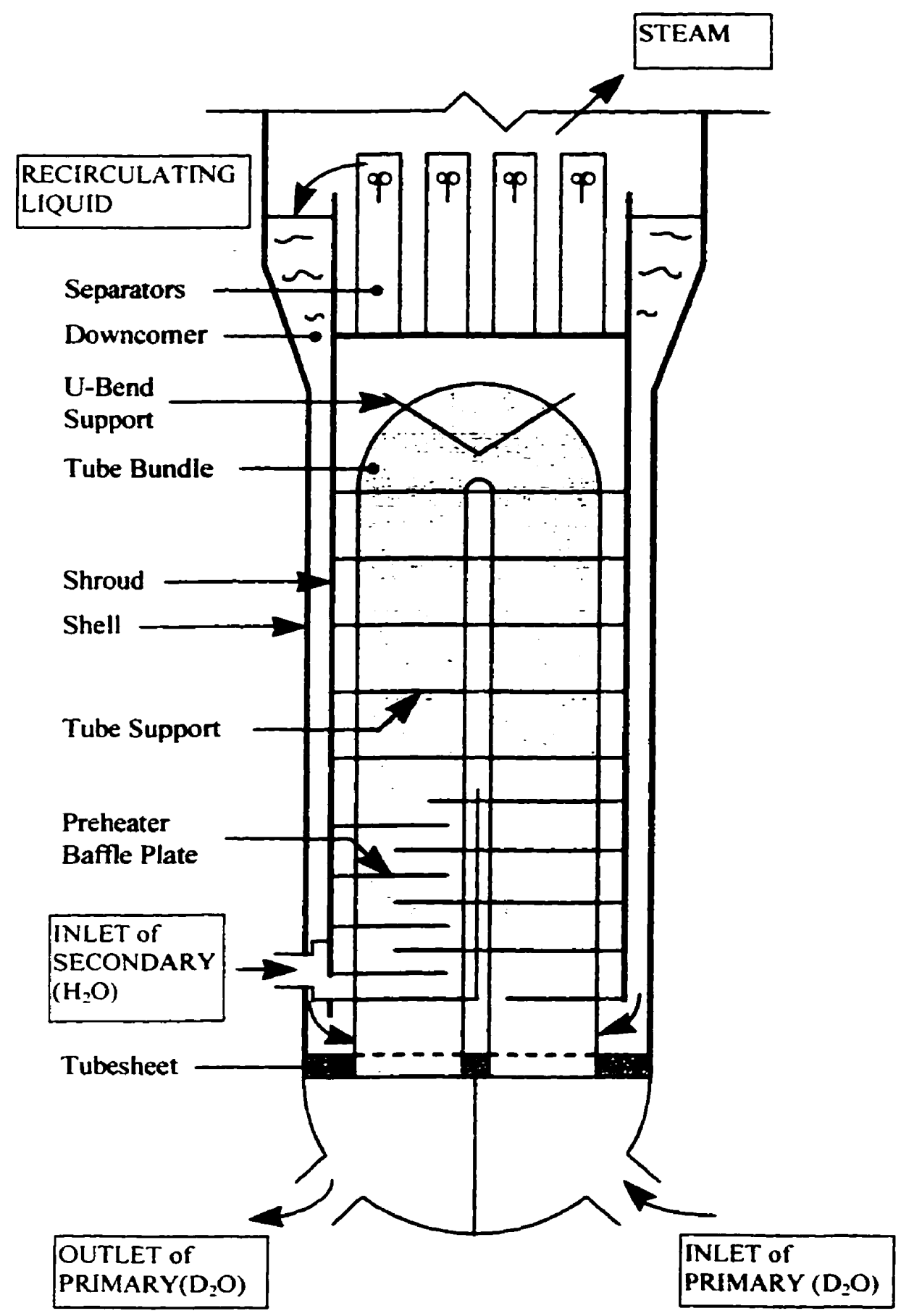


Figure 1.3: Bruce A steam generator \& steam drum
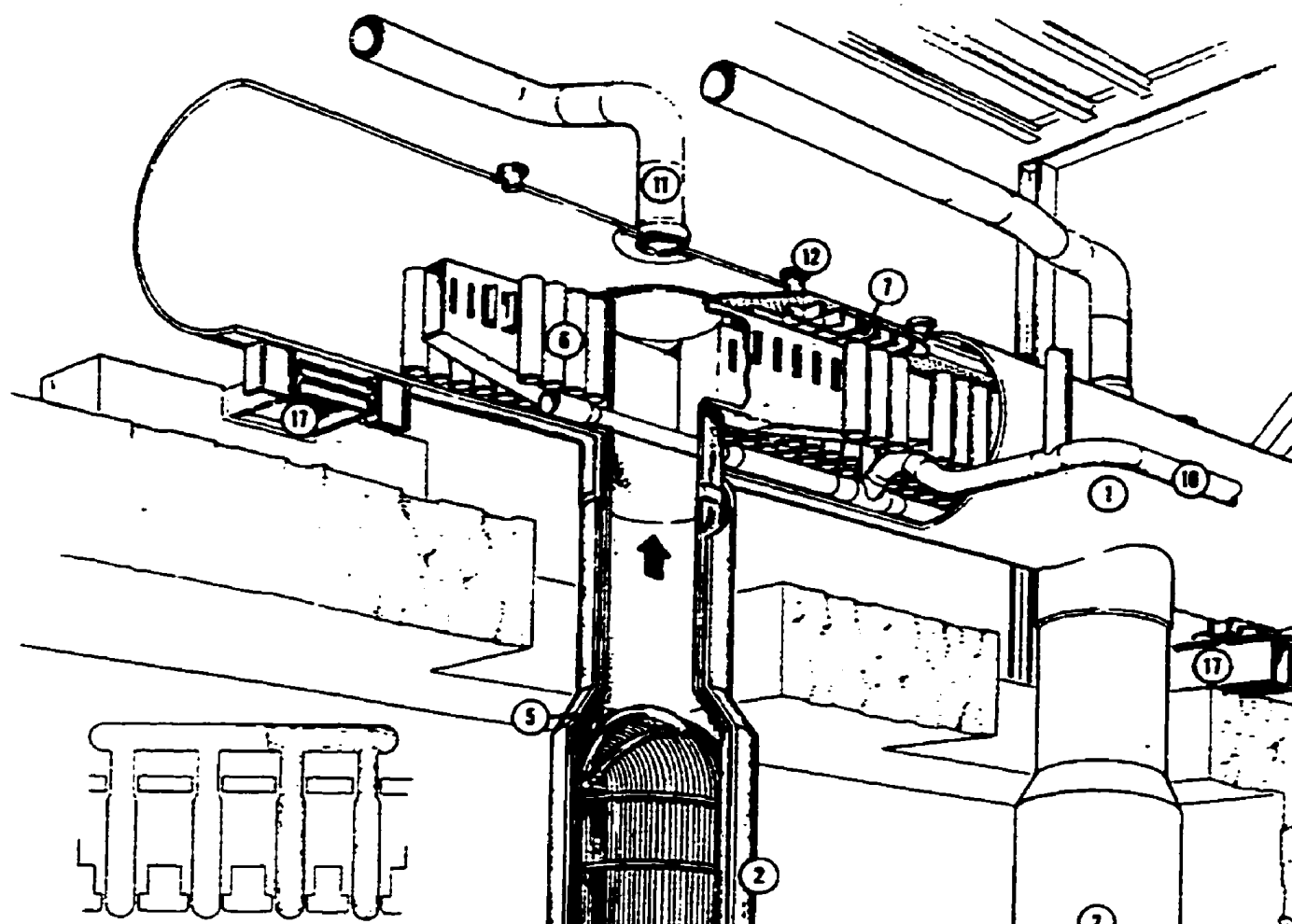

steam drum

2 steam generator

3 heavy water inlet

4 heavy water outlet

5 downcomer annulus

6 cyclone separators

7 steam scrubber

8 blow-down piping

9 16" manway

10 feedwater inlet

11 steam outlet

12 safety valve nozzles

13 preheater

14 steam generator support

15 support hangers

16 boiler vault seal

17 drum seismic restraints

18 steam generator seismic restraints 
Figure 1.4: Darlington steam generator with integral preheater

steam drum

2 steam generator

3 heavy water inlet (2)

4 heavy water outlet

5 downcomer annulus

6 primary cyclones

7 secondary cyclones

8 blow-down nozzle

9 manway

10 main steam outlet nozzles

11 preheater

12 steam generator support

13 containment seal bar/skin

14 lower lateral restraint lugs

15 preheat condensate retum nozzle

16 feedwater nozzle

17 upper lateral restraint lugs

18 ring beam

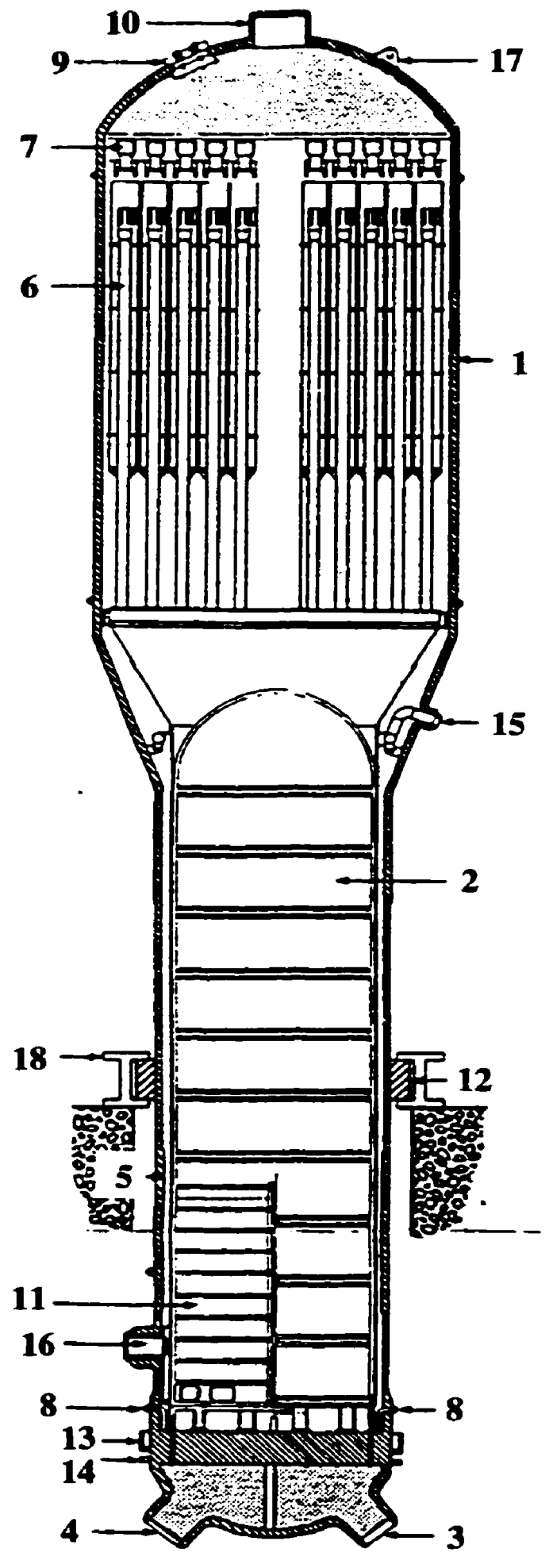


Figure 1.5: Tube support designs (including U-bend supports)

\section{HORIZONTAL SUPPORTS}

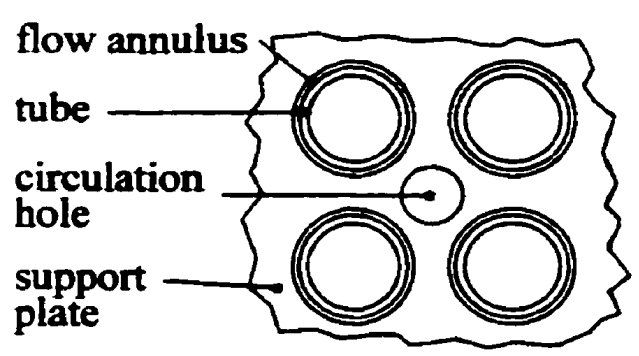

Drilled hole

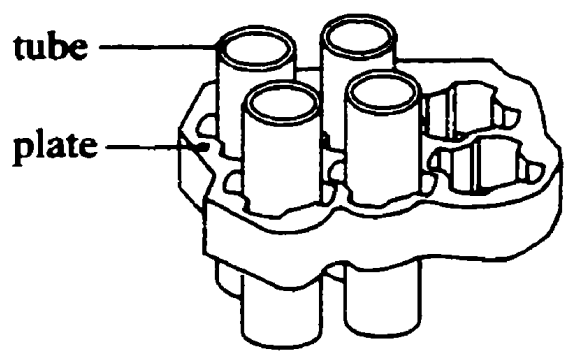

Quatrefoil (4 lands)

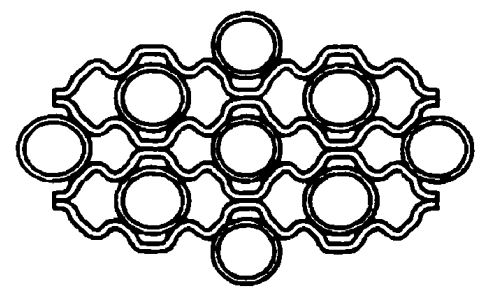

Formed bars

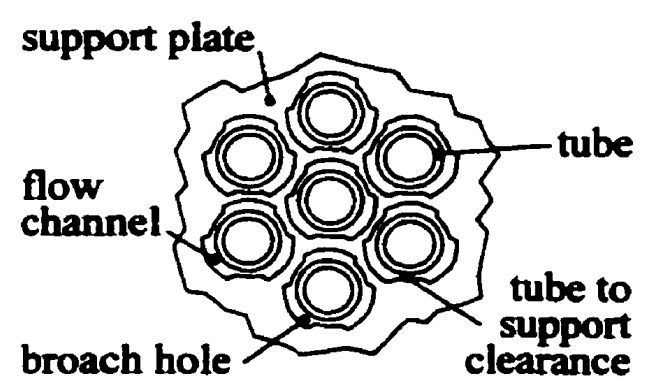

Trilobar or trefoil (3 lands)
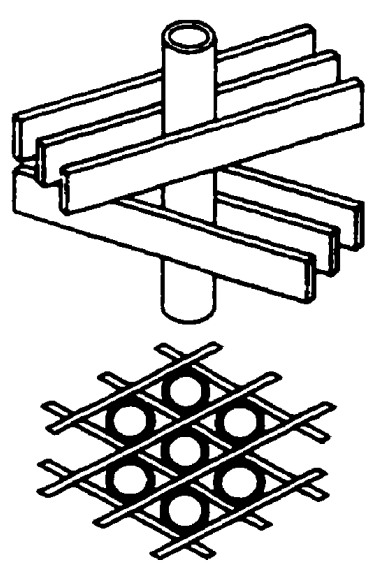

Lattice bar or egg crate 
Figure 1.5 (continued)

Arrangement of Darlington Lattice Bars [12]

every $8^{\text {th }}$ bar

is a "high bar"
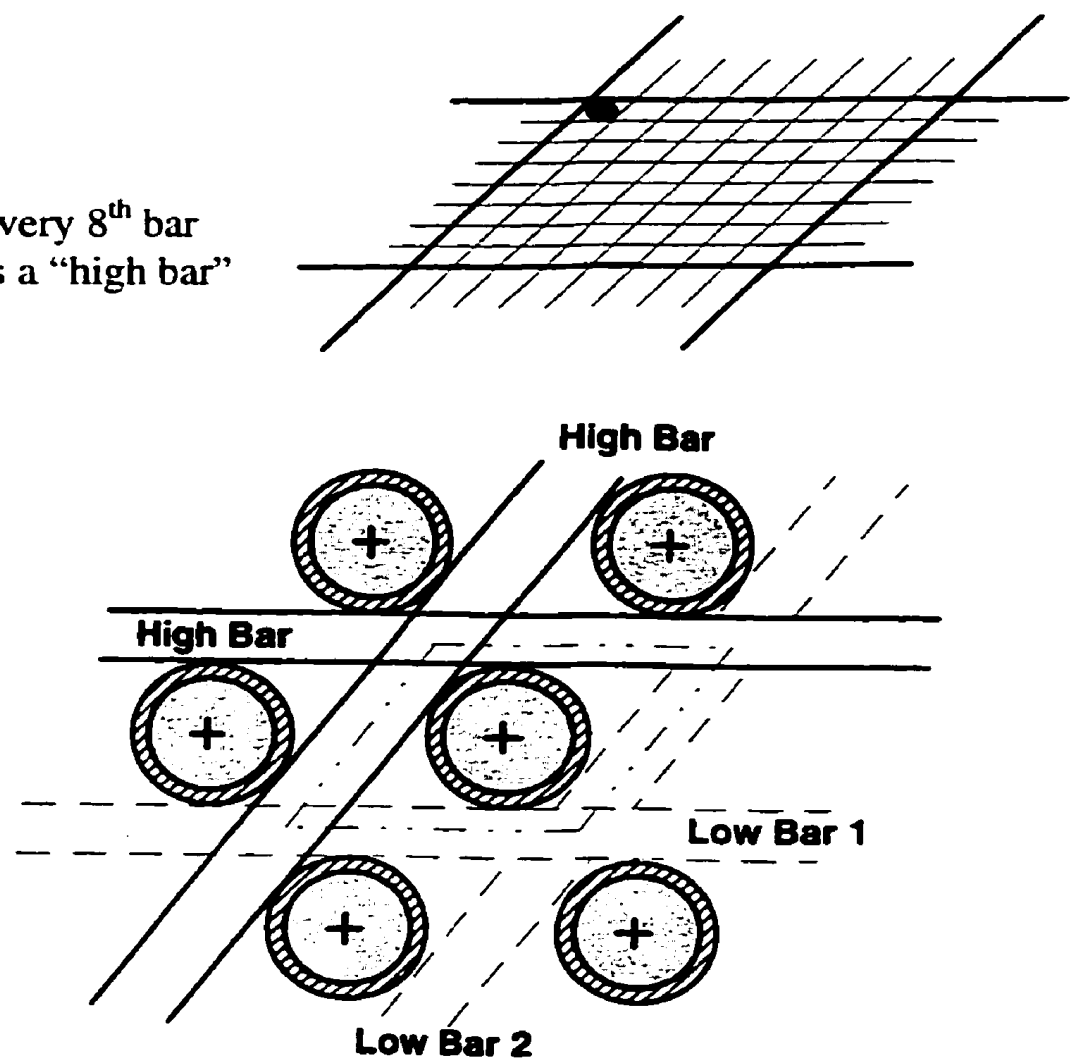

Top View of the lattice bar support configuration

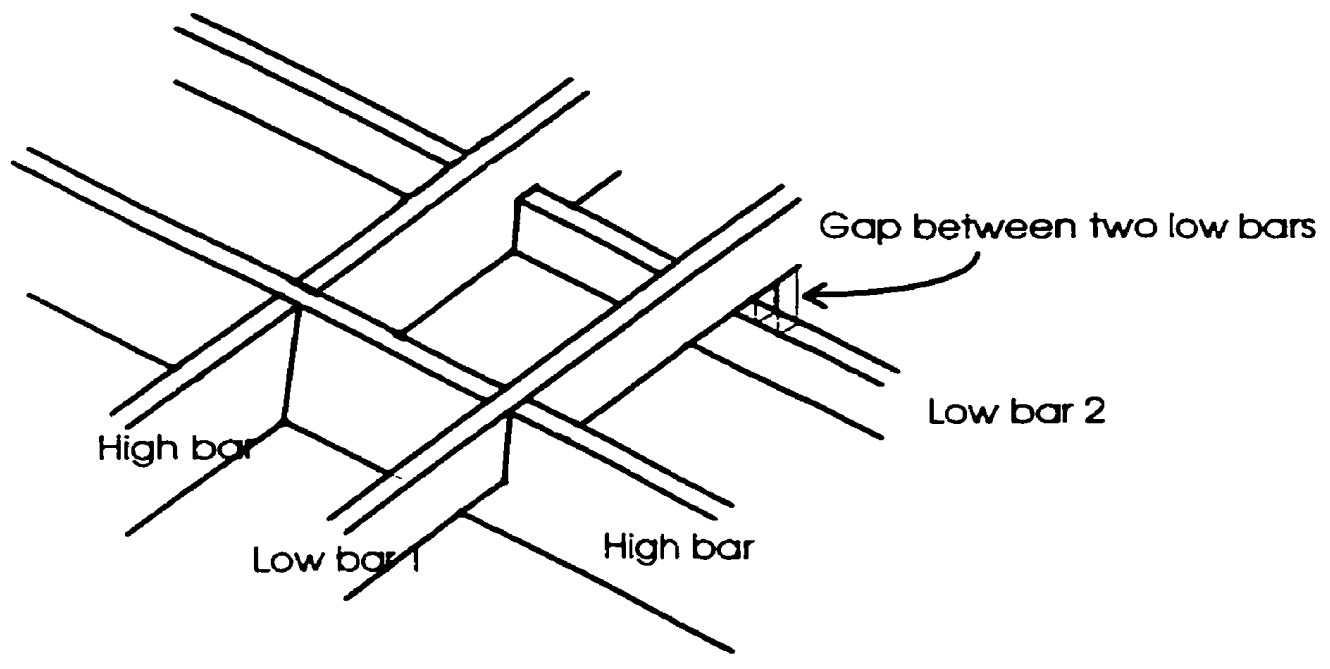

Lattice bar support configuration at the corner of the $8 \times 8$ cell 
Figure 1.5 (continued)

\section{U-BEND REGION SUPPORTS}

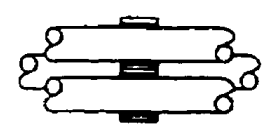

scalloped bars

ह 8

wiggle bars

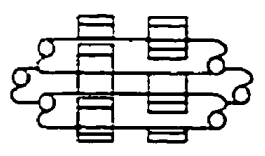

lattice bars

(triangular array)

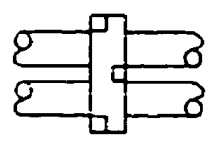

lattice bars

(in-line array)

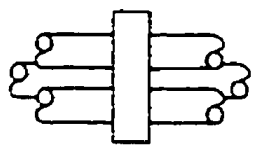

flat bars

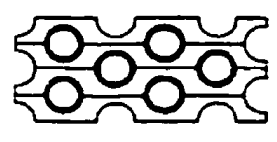

2000
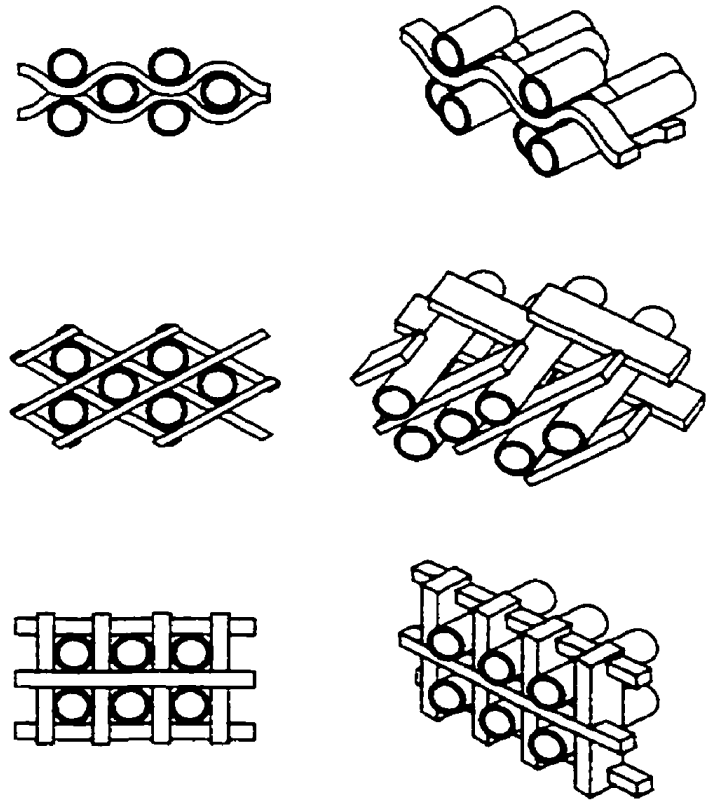

aboga 


\section{Chapter 2}

\section{LITERATURE SURVEYS FOR FOULING OF SG SUPPORTS}

\subsection{Experience with Support Fouling}

The following sections describe occurrences of support fouling in existing steam generators or in laboratory mock-ups.

\subsubsection{Experience at Crystal River}

One of the first reports of the blockage of broached supports (shown in Figure 1.5) came from Florida Power Corporation's Crystal River Unit 3 nuclear power station, which uses once-through steam generators (OTSGs) [1,2]. A steady increase in the pressure drop was noted across the first 9 of the 15 broached support plates, from the start of commercial operation in 1977. By the summer of 1984 , the resistance to flow in the tube bundle resulted in the feedwater backing up and flooding other components. From then on, the feedwater flow and consequently the unit power output needed to be curtailed on a continuous basis.

The increase in pressure drop was attributed to deposition of corrosion products causing increased restrictions of the flow paths through the supports. Visual inspection of the SGs showed that the $1^{\text {st }}$ and $6^{\text {th }}$ support plates had light blockage (0 to $10 \%$ ), the $3^{\text {rd }}$ and $5^{\text {th }}$ support plates were moderately blocked (20 to $50 \%$ ), and blockage peaked at the $4^{\text {th }}$ TSP (50 to $90 \%)$.

As illustrated in Figure 2.1, the blockage consisted of "lips" protruding from the support towards the tube surface, both at the inlet and outlet. Blockage at the inlet face was typically greater than at the outlet. Rippled deposits or "mini-lips" were also observed on 
all the surfaces within the broach hole. The deposits consisted primarily of magnetite, with 2 to $3 \%$ each of metallic copper, nickel, and chromium.

Blockage would not cause flow oscillations in OTSGs, as the flow is by pumped forced convection and not thermosyphoning; however, the blockage at Crystal River was unacceptable from the standpoint of forcing station derating. Attempts were made in May 1985 to manage the flow blockage problem by hydraulic cleaning to remove deposit. $\mathrm{A}$ visual inspection performed only half a year later showed that lip growth and blockage had returned to the condition observed prior to hydraulic cleaning.

The regions of extensive fouling had steam qualities in the range of 30 to $50 \%$. Fouling was reduced beyond the $6^{\text {th }}$ support probably due to depletion of the liquid phase (which is the particle-bearing phase), or due to reduced particle concentration, or both.

\subsubsection{Experience at Westinghouse SGs}

Flow oscillations and blockage have been reported in some Westinghouse SGs with quatrefoil supports (shown in Figure 1.5). A survey of plants with quatrefoil supports showed a correlation between the occurrence of broach-hole deposition and plant operation at the low end of the desirable $\mathrm{pH}$ range (i.e., $\mathrm{pH}<9.3$ ) [3]. ${ }^{2}$

Inspections showed that deposits had a lipped appearance similar to those of the Crystal River SGs. The lips had grown along the entire circumference of the broach hole, as well as on the tube surface. [Japanese stations using Westinghouse SGs, i.e., quatrefoil supports, also reported such a lip formation, with a larger lip on the support side.]

\footnotetext{
2 The SG water $\mathrm{pH}$ is kept in a narrow band in the alkaline range to discourage corrosion. Additives such as morpholine or ammonia are used for $\mathrm{pH}$ control, phosphates are used as buffers*, and hydrazine $\left(\mathrm{N}_{2} \mathrm{H}_{4}\right)$ as an oxygen scavenger. Chemistry control affects fouling in that poor control leads to greater rates of corrosion and hence a greater mass transport of corrosion products.
} 
Field observation and lab experience suggested that colloids* formed the deposits, which is consistent with the smooth, hard deposit morphology. In general, preferential growth was observed in the quatrefoil design compared to the drilled hole design, on the support side of the broach holes, and on the bottom face of the support.

\subsubsection{Experience at Bruce A}

Ontario Hydro has reported blockage of the trefoil broached plate supports at the Bruce A station [4]. Bruce $A$ is a 4-unit CANDU nuclear power station, in service since 1976, whose RSGs operate with a mixed iron/copper feed-train. A program of copper removal from the feed-train is currently in progress, as copper is known to be a deposit hardening agent. As discussed in Chapter 1, the flow blockage problem was first revealed as a level oscillation in the horizontal steam drum that is connected to four SGs in Unit 2. An analysis indicated that the oscillation was most likely caused by flow blockage in the upper region of the SGs [4]. ${ }^{3}$ Later, most of the boilers in Unit 1 also experienced flow oscillations.

Inspections in Unit 2 Boiler 3 showed that the blockage was caused by deposition of corrosion products in the broach holes of the supports [5]. The extent of blockage increased with support plate elevation; it was also greater on the hot leg* (whose quality ranges from $2-3 \%$ at the bottom to $\sim 30 \%$ at the U-bend region) than the cold leg* (quality $0 \%$ at the bottom to $\sim 20 \%$ at the U-bend). Typical deposits revealed by remote inspection are shown in Figure 2.2 [5,6]; they were found to be smooth, fine, amorphous*, and very hard (i.e., not easily removed even by water-lancing).

A visual inspection of Bruce A Unit 4 SGs showed the blockage to be more uniform than in Unit 2, ranging from $30-40 \%$ at the $3^{\text {rd }}$ support to $40-60 \%$ at the $7^{\text {th }}$ support, and little

\footnotetext{
${ }^{3}$ In a recirculating system driven by thermosyphoning, high flow resistances in the two-phase (steam/liquid) region will tend to destabilize the flow.
} 
difference between the hot leg and the cold leg. In all cases, the deposits were brittle and had lips at the inlet of the support. In contrast. Unit 1 had a high volume of deposit all over, with cold leg worse than the hot leg, and with soft and easily water-lanced deposits.

\subsubsection{Experience at Bruce B}

Although the SGs are of a similar design, visual inspections at the Bruce $B$ site have shown relatively litule blockage [3]. Only small lips of deposit at the rims of the broach holes in the hot leg have been observed. No flow oscillations were experienced.

One significant difference is that the stations at the B site consist of all-ferrous feedwater systems (i.e., no copper), and are operated at a higher $\mathrm{pH}$ than the stations at the $\mathrm{A}$ site. Bruce $B$ also has a history of better chemistry control than Bruce A.

\subsubsection{Experience at Pickering B}

Visual inspection of Ontario Hydro's Pickering B NGS Unit 5 Boiler 4 was carried out in the early 1990's [5]. The upper $\left(5^{\text {th }}, 7^{\text {th }}\right.$, and $\left.9^{\text {th }}\right)$ trefoil broached-plate supports were inspected and their deposits were found to be very thin and fine; some of the broach holes on the $5^{\text {th }}$ support showed incipient lips. In contrast, deposits on the $1^{\text {st }}$ support plate were thick and irregular in shape, crystalline, hanging from the underside of the plate, and so thick that most broach holes were blocked.

Flow oscillations of the type experienced at Bruce A were not observed at Pickering B, as the blockage was restricted to the single-phase liquid region at the bottom of the SG. Contrary to the destabilizing effect of high flow resistance in two-phase regions, high resistance in the single-phase region tends to stabilize the flow. However, the flow rate would still be reduced. 


\subsubsection{Experience in a Fouling Test Loop}

Fouling experiments conducted in recent years at CRL involved a broached support subjected to a flow of $0.25 \mu \mathrm{m}$ magnetite particle suspension at high temperature and pressure (representative of SG conditions) for 600 continuous hours [3]. The fluid was heated to saturation at the inlet; no additional heat was added in the test-section. [Any steam present would have been the result of pressure loss effects.] The bifoil support had a geometry similar to the trefoil Bruce broached plate, but had an artificially large flow area ratio* between the free-span region* and the support region.

As shown in Figure 2.3, small clumps of deposits formed within the broach holes; these deposits were heaviest at the hole inlet. Scanning electron microscope photos (not shown here) indicated that the deposits were amorphous.

An interesting facet of the fouling problem is that several research groups have attempted to artificially foul a support and have found very little, if any, deposit. At CRL, a testsection with trefoil supports was run recently at SG conditions for 500 hours, and no significant deposit was obtained even after the concentration of solubles was increased, or particles were added, or mass flux was boosted (by increasing the area ratio between freespan and support). Likewise, a Japanese group ran a similar 3000 hour test and obtained no significant deposit [3].

\subsection{Studies on Support Thermalhydraulics}

For understanding of the relationship between fouling and the thermalhydraulic environment of SG supports, information was sought on characteristic flow patterns near such supports. The majority of papers related to this topic focus on corrosion and flowinduced vibrations (see, for example, the review by Green [7]). However, a few were found that describe flow patterns. 
Studies conducted at Winfrith (U.K.) around 1977 investigated the wake geometry of air/water bubbly flow past a blockage [8]. The length and center of flow recirculation zones could be measured directly with the use of a transparent test-section. These parameters were in good agreement with predictions from a computer code that modeled velocity vectors.

In 1979, Bates et al. at the Battelle Pacific Northwest Laboratories [9] measured local velocities near circular-hole type support plates to characterize the flow patterns associated with tube denting and degradation. This was followed by computer simulations of void and velocity fields near the support plates [10]. The researchers could not find any solid link between flow patterns and tube denting, but speculated that tube eccentricity might play a role.

Between 1979 and 1981, Sekoguchi et al. at Kyushu University in Japan [11] studied flow pattems near obstructions, using both air/water and steam/water mixtures. The results revealed local thinning of liquid films very close to the obstruction, which could be linked to alternating dryout and rewetting of the tube surface observed in the heated tests. This suggested a potential for accelerated corrosion of the tube near the support, but did not shed much light on fouling mechanisms.

In 1985, Caille et al. at the University of Central Florida [12] used air/water mixtures to visualize flow near supports of circular hole and trefoil designs, to determine where vapour blanketing (and hence accelerated corrosion) could occur. The resulting flow patterns, featuring oscillations and periods of reverse flow, could not be categorized according to known flow regimes. The study was inconclusive with regard to fouling.

A 1983 EPRI (Electric Power Research Institute) report by Cassell \& Vroom [13] offered the most useful information, as it describes experiments performed with a broached plate under high-pressure and high-temperature SG conditions. The steam/water flow upstream was found to be unperturbed by the support, except for slight necking, while 
flow downstream was characterized by jetting, swirling, and recirculation, the intensity of which depended on the velocity through the support.

\subsection{Summary of Literature Survey}

In summary, literature exists on the occurrence of deposits on various supports in various types of SGs, and on the thermalhydraulic environment near SG supports. No information could be found that described the relationship between the fouling and flow patterns. Also, nothing was found that explained the process by which SGs such as those at Bruce $A$ could become incapacitated by support fouling.

The next Chapter describes the key components of the support fouling research program at CRL, and the bases on which decisions were made.

\section{References}

[1] L.E. Johnson, "Fouling in Once-Through Steam Generators", Paper 87-WANE12, ASME Winter Annual Meeting, Boston, Mass., U.S.A., 1987.

[2] R.H. Thompson and L.S. Lammana, "Video Inspection and Sampling of the Crystal River Unit-3 Once-Through Steam Generators", Proceedings of the $47^{\text {th }}$ Int. Water Conference, Pittsburg, Pa, USA, 1986 October 27-29.

[3] C.W. Turner, private communication at AECL-CRL, 1996 and 1998. 
[4] R. Dyck, P. Spekkens, A. Marchand and K. Verma, "Operational Experience with Steam Generators in Canadian Nuclear Power Stations", Proceedings of the $1^{\text {st }}$ Steam Generator and Heat Exchanger Conference (sponsored by the Canadian Nuclear Society), 1990 April 30 to May 2, Toronto, Canada, p. I-10.

[5] "Visual Inspection \& Trial Waterlancing of Bruce and Pickering Boilers", video produced by H. Carbajales at Ontario Hydro, 1990 June; also private communication with H. Carbajales, 1991.

[6] R.V. Murphy and P.W. Reynolds, "Monitoring Steam Generator Broach Hole Blockage using Penetrating Radiation. Gamma Rays: Theory of Single Source / Detector”, protected COG report No. COG-91-212-1, 1991 November.

[7] S.J. Green, "Thermal, Hydraulic, and Corrosion Aspects of PWR Steam Generator Problems", Heal Transfer Engineering, Vol.9, No.1, pp. 19-67 (1988).

[8] D.P. Robinson and R.A.K. Smith, "Water Modelling Studies of the Hydrodynamic Characteristics of Impermeable Blockages in an 11-Pin Geometry", Atomic Energy Establishment Winfrith (U.K.) Report No. AEEW-M1471, 1977 March.

[9] J.M. Bates, C.W. Stewart and A.M. Sutey, "Experimental Study of Single-Phase Flow Fields Around Steam Generator Tube Support Plates", Proceedings of the Winter Annual Meeting of the ASME, 1979 December 2-7, pp.41-53.

[10] C.W. Stewart, M.J. Thurgood, and D.W. Mayer, “Analysis of Single- and TwoPhase Flow Fields Around PWR Steam Generator Tube Support Plates", Report No. EPRI-NP-1162, 1979 August. 
[11] K. Sekoguchi et al., "Gas-Liquid Two-Phase Flow in Double-Tubes-AnnularPassage with Flow Obstruction", Bulletin of the JSME, Vol.24, No.189, (1981 March).

[12] G.W. Caille, E.R. Hosler and F.S. Gunnerson, "Visual Observations of Flow Patterns Through Tube Support Plates of Circular Hole and Trefoil Designs”, A.I.Ch.E. Symposium Series No.245, V.81, pp.148-155 (1985).

[13] D.S. Cassell and D.W. Vroom, "Thermaihydraulic Tests of SG Tube Support Plate Crevices”, Report No. EPRI-NP-2838 (Volumes 1-3), 1983 January. 
Figure 2.1: Deposits on Crystal River supports [2]

(a) Typical support deposits ( $3^{\text {rd }}$ and $4^{\text {th }}$ supports)

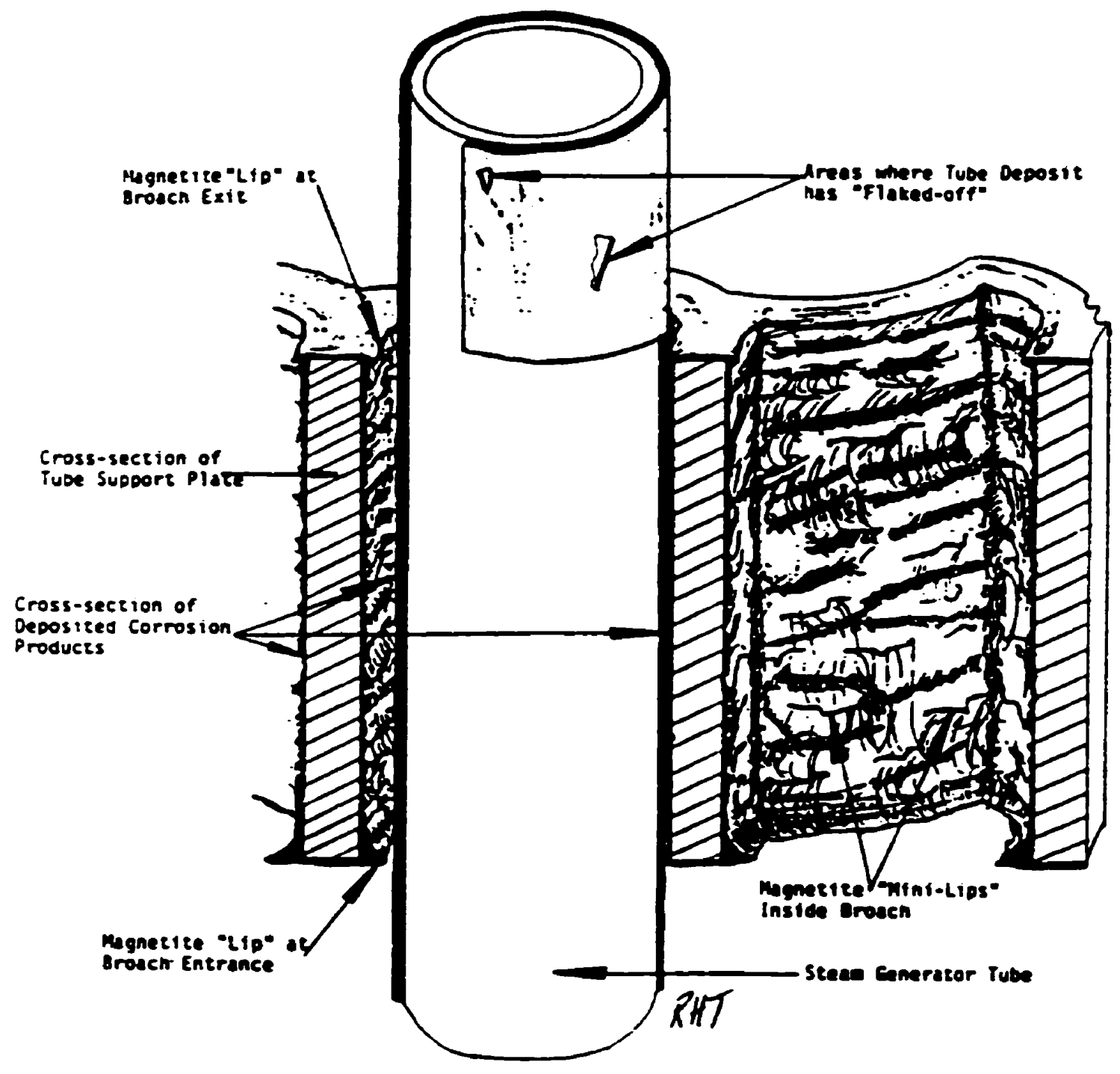


Figure 2.1 (continued)

(b) Typical tube deposits ( $5^{\text {th }}$ and $6^{\text {th }}$ supports)

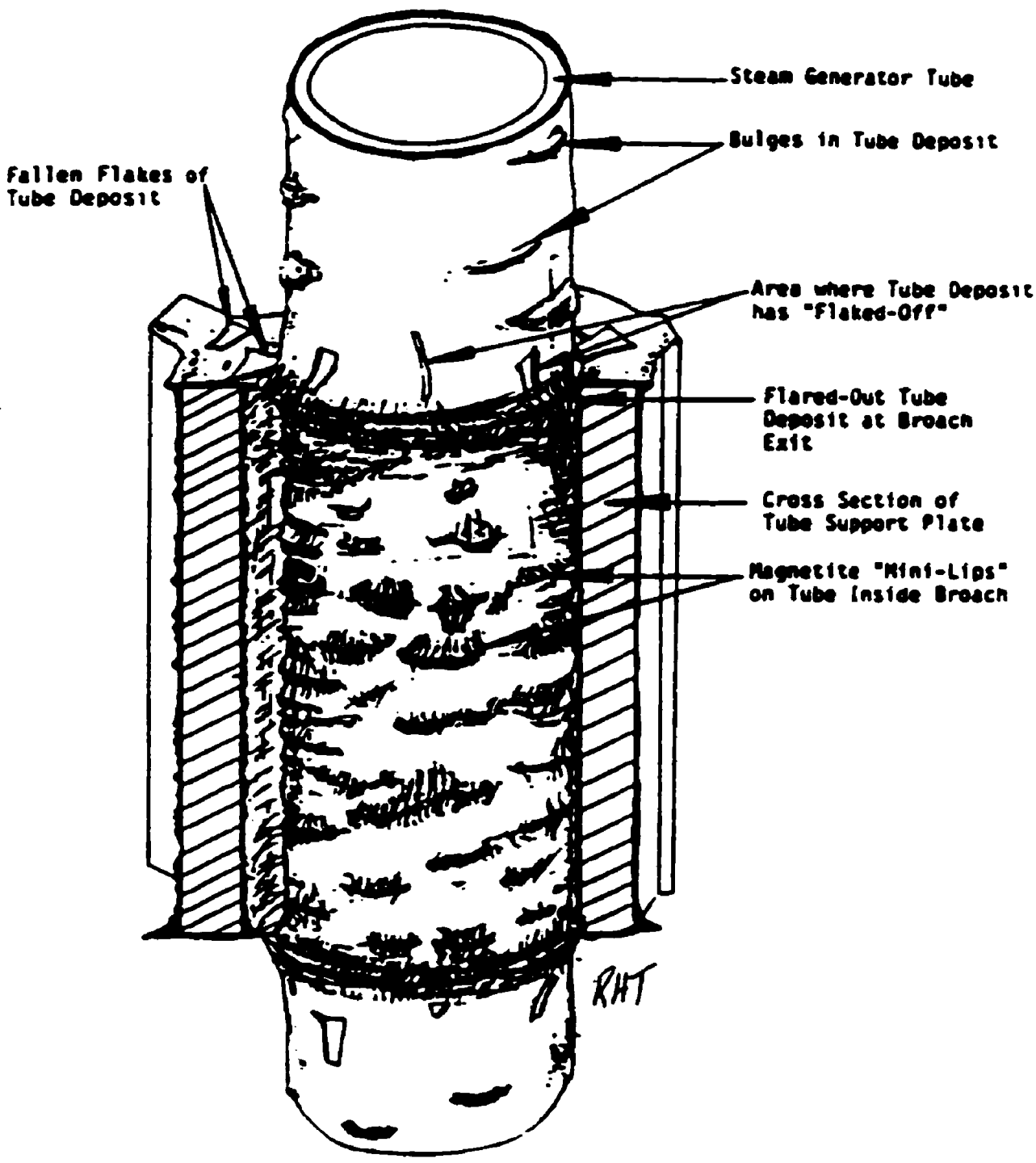


Figure 2.2: Deposits on Bruce A Unit 2 Boiler 3 supports [5,6]

(a) broached plate $\# 5$ (partially fouled)

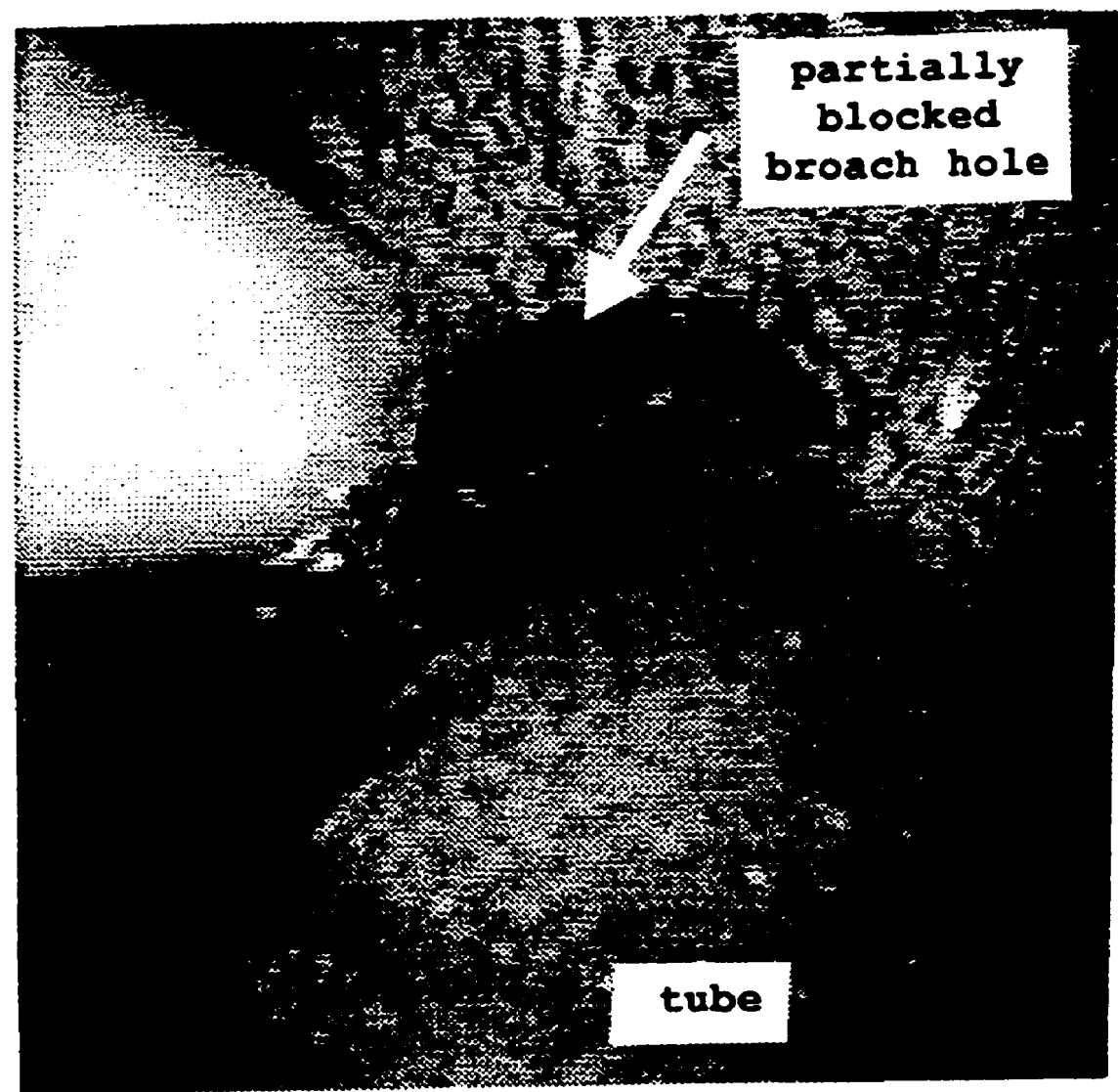


Figure 2.2 (continued)

(b) broached plate $¥ 6$ (heavily fouled)

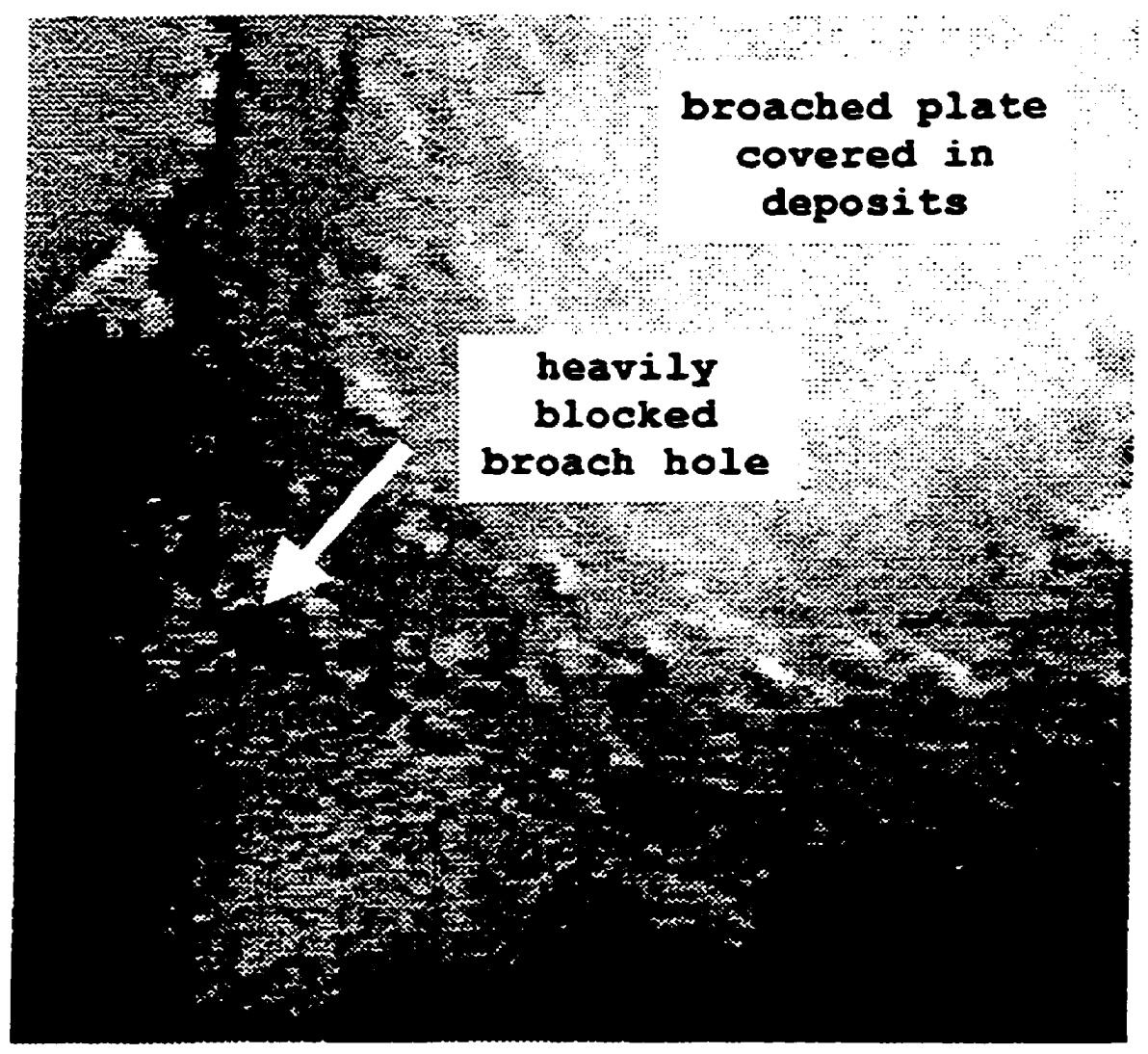


Figure 2.3: Deposition on experimental bifoil support

\section{OUTLET}

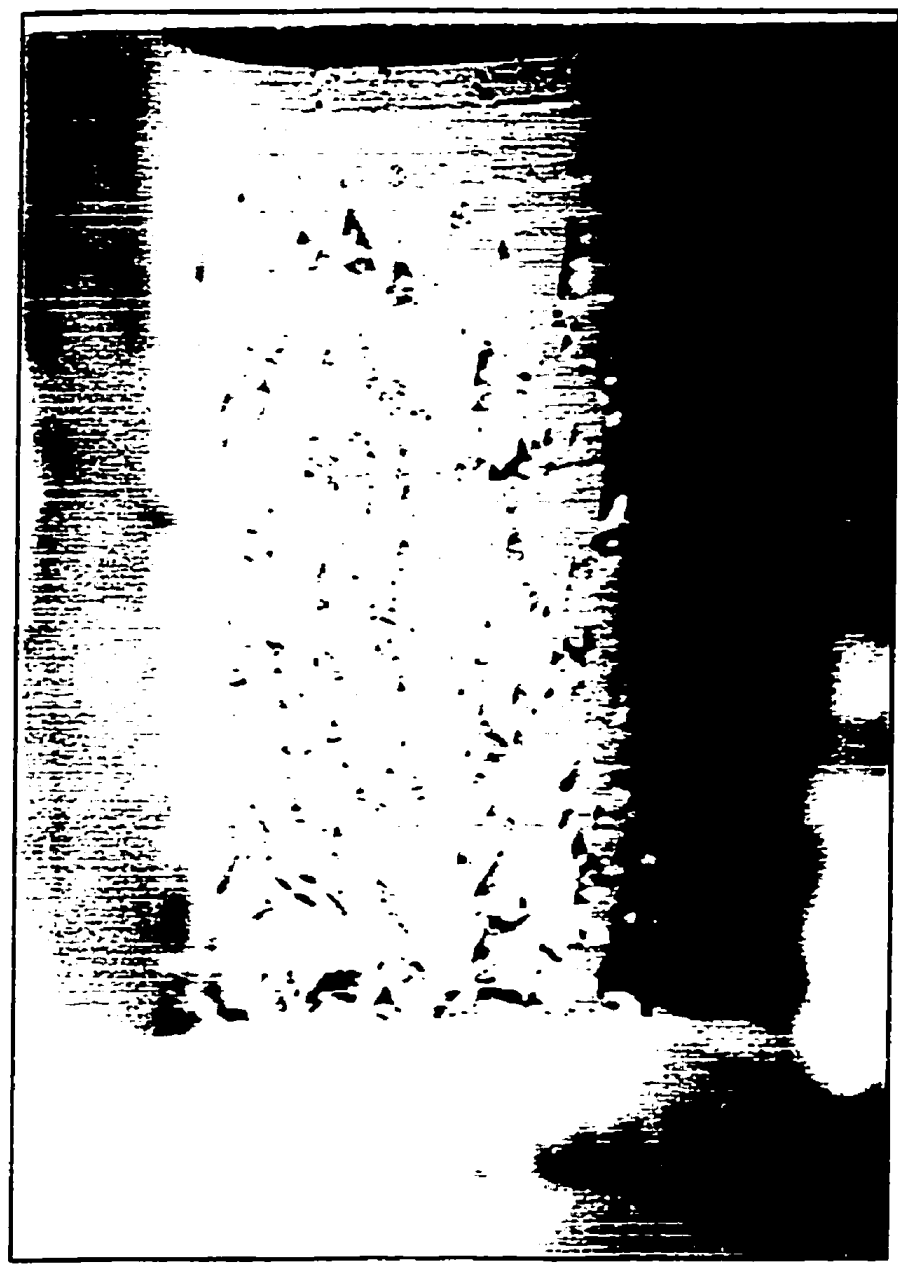

FLOW INLET

(Scale: support is 1 inch or $25 \mathrm{~mm}$ tall) 


\section{Chapter 3}

\section{DEVELOPING A RESEARCH PROGRAM}

\subsection{Experiments}

An experimental research program was funded at AECL's CRL to investigate more closely the thermal and hydraulic environment near a support. This program, managed and directed by the author, evolved over a five-year period, from 1991 to 1996.

As described in Chapter 4, tests were first carried out using air/water mixtures under ambient conditions, i.e., room temperature and near-atmospheric pressure. Because these could only show hydraulic effects, tests designed to show the thermal effects were subsequently carried out using liquid/vapour mixtures of Freon-11 or $\mathrm{CCl}_{3} \mathrm{~F}$ (Chapter 5). Table 3.1 shows that the Freon fluid is also more representative of the SG fluid than air/water under ambient conditions, in terms of liquid/vapour density ratio and vapour bubble size. One flow visualization experiment was done under SG conditions, for confirmation purposes (see Section 5.6).

The experimental test-sections were scaled up by 20 to $50 \%$ for better observation and for better accessibility by instruments. The flow area ratios between the free span* and the support channel were representative of the flow area ratios in the SGs. Likewise, in the support region, the height to channel hydraulic diameter (or $L / D_{\text {hy }}$ ratio) was matched. The need to keep these two parameters representative was made clear during early trial runs, in which an improperly scaled test-section showed obviously unrealistic flow patterns.

The first useful test-section consisted of a half-rod representing a SG tube cut lengthwise, and its associated Bruce-type broached plate. The rod and plate were attached to a 
transparent acrylic casing whose flat wall allowed observation of flow within the broach hole. While this test-section was too simple to show representative flow patterns upstream and downstream of the plate, it was the only one with a sectioned broach hole, and from this perspective gave much useful insight on flow within the support.

Subsequent test-sections for water tests consisted of 7 full rods in a circular pipe and 6 full or partial rods in a rectangular pipe. By the time a test-section was needed for Freon tests, it was established that flow patterns were influenced more by whether or not area ratio and $L / D_{\text {hy }}$ were conserved, than by the presence or absence of neighboring rods or tubes, as discussed in Section 4.2. The Freon tests therefore used only 1 full rod in a circular pipe.

As flow visualization was an important part of the work, various methods were tried. Some techniques, such as dye injection, entrained particles, or hydrogen bubble generation, were unsuccessful, because they were either better suited to low (laminar) flow rather than the turbulent flow found in SGs, or they did not lend themselves readily to flow tracking. For the water tests, the most successful technique involved introducing air bubbles into the flow. While it was tempting to assume that the bubbles represented steam, the vastly differing properties of low-pressure air and high-pressure steam meant that the air could be regarded only as a flow visualization tool. For the Freon tests, the vapour bubbles used as a tool were considered more representative of steam bubbles, as indicated in Table 3.1 .

\subsection{Analytical Modeling}

A computer code called SLUDGE was developed at CRL in the late 1980's to predict the deposition of particles throughout the SG [1]. The local thermalhydraulic conditions (e.g., mass flux, quality, pressure) were computed by the THIRST code [2] and fed into the SLUDGE code. SLUDGE then calculated the magnetite particle concentration, and 
the extent of deposit buildup on tubes, supports, and the tube sheet at the bottom of the steam generator. This macroscopic code modeled the supports simply in terms of porosity*. The correlations and expressions used in SLUDGE to predict deposition are described in Appendix A.

The SLUDGE code could not predict the extensive support fouling at, for example, the Bruce A station. A tool was needed to more accurately predict local deposition. Using this tool, supports could be assigned a "fouling propensity factor" based on their design, which could then be used in SLUDGE in the calculation of support deposition rates. This concept gave rise to the TSFOUL (Tube-Support FOULing) computer program, described in Chapters 7 to 9.

\subsection{Challenges}

The main challenge for all laboratory research for SGs is the expense and complexity of representing the high-pressure, high-temperature conditions of the SG. Much work is therefore typically undertaken under ambient conditions and rationalized with the help of scaling laws or parameters (such as similar liquid/vapour density ratios). The assumptions are then confirmed in a limited number of difficult tests under the more extreme conditions. Likewise, the models used in numerical simulations are often validated with ambient-condition data, and assumed to apply to SG conditions.

Even the use of a modeling fluid such as Freon-11, chosen for its convenient boiling point near room temperature, poses challenges. This fluid is considered an ozone-depleting substance, and new environmental regulations in effect in 1994 led to very tight controls on, for example, test loop leakage and recovery of the loop inventory after use. Freon-1 1 is currently banned as a test fluid at CRL. 
Another challenge in this work in particular is that various aspects of tube-support fouling were investigated in parallel by different research groups. For example, while the flow pattern investigation has now been completed, trials to artificially foul a broached plate in the laboratory and to measure fouling rates are still ongoing. Thus, many of the fouling mechanisms proposed in Chapter 6 and the deposition rates obtained in Chapter 10 remain to be verified.

The next Chapter describes experiments carried out with ambient-conditions water as modeling fluid.

\section{References}

[1] Y. Liner, M.B. Carver, C.W. Turner, and A.O. Campagna, "Simulation of Magnetite Particulate Fouling in Nuclear Steam Generators", Proceedings of the ASME meeting on Steam Generator Sludge Deposition in Recirculating and Once Through Steam Generator Upper Tube Bundle and Support Plates, NE-Vol.8, Book No. G00690, 1992.

[2] M.B. Carver, L.N. Carlucci, and W.W.R. Inch, "Thermal-hydraulics in Recirculating Steam Generators. THIRST Code User's Manual", AECL Report No. AECL-7254, 1981.

[3] J.G. Collier, Convective Boiling and Condensation, $2^{\text {nd }}$ edition, McGraw-Hill Book Company, 1981, p.121. 
Table 3.1: Comparison of physical properties: SG steam/water, air/water, Freon-1 1

\begin{tabular}{|c|c|c|c|c|}
\hline Fluid & $\begin{array}{c}\text { vapour/ } \\
\text { liquid } \\
\text { steam/water } \\
\text { [actual SG } \\
\text { conditions] }\end{array}$ & $\begin{array}{l}\text { vapour / } \\
\text { liquid } \\
\text { Freon-11 } \\
\text { [Freon loop } \\
\text { conditions] }\end{array}$ & $\begin{array}{l}\text { vapour / } \\
\text { liquid } \\
\text { Freon-11 } \\
\text { [Freon loop } \\
\text { conditions] }\end{array}$ & $\begin{array}{l}\text { gas/liquid } \\
\text { air/water } \\
\text { [water loop } \\
\text { conditions] }\end{array}$ \\
\hline pressure [MPa] & 4.4 & $0.2^{*}$ & 0.1 & 0.1 \\
\hline temperature $\left[{ }^{\circ} \mathrm{C}\right]$ & $256=T_{\text {sax }}$ & $44.6=T_{\text {sat }}$ & $23.7=T_{\text {sat }}$ & 23.7 \\
\hline liquid density $\rho_{\mathrm{f}}\left[\mathrm{kg} / \mathrm{m}^{3}\right]$ & 790.7 & 1444.9 & 1495.2 & 997.2 \\
\hline gas density $\rho_{\mathrm{g}}\left[\mathrm{kg} / \mathrm{m}^{3}\right]$ & 22.2 & 11.0 & 5.75 & 1.17 \\
\hline liq:gas density ratio $\rho_{\mathrm{f}} / \rho_{\mathrm{g}}$ & $36: 1$ & $131: 1$ & $260: 1$ & $852: 1$ \\
\hline liquid viscosity $\mu_{\mathrm{f}}[\mathrm{kg} / \mathrm{m} . \mathrm{s}]$ & $1.05 \times 10^{-4}$ & $3.53 \times 10^{-4}$ & $4.25 \times 10^{-4}$ & $9.17 \times 10^{-4}$ \\
\hline surface tension $\sigma\left[\mathrm{kg} / \mathrm{s}^{2}\right]$ & 0.0248 & 0.0151 & 0.0176 & 0.0722 \\
\hline $\begin{array}{l}\text { liquid } \operatorname{Re} \text { at } u_{\mathrm{f}}=1 \mathrm{~m} / \mathrm{s} \text { and } \\
D_{\text {hy }}=5 \mathrm{~mm}[-]^{* *}\end{array}$ & $3.8 \times 10^{4}$ & $2.0 \times 10^{4}$ & $1.6 \times 10^{4}$ & $0.5 \times 10^{4}$ \\
\hline $\begin{array}{l}\text { bubble size term } \sigma /\left(\rho_{\mathrm{r}}-\rho_{\mathrm{g}}\right) \\
{\left[\mathrm{m}^{3} / \mathrm{s}^{2}\right]^{* * *}}\end{array}$ & $3.2 \times 10^{-5}$ & $1.1 \times 10^{-5}$ & $1.2 \times 10^{-5}$ & $7.3 \times 10^{-5}$ \\
\hline
\end{tabular}

(See Nomenclature at beginning of thesis for description of variables.)

* This is the maximum allowable pressure for non-registered "pressure vessels" used in test loops at CRL.

** The Reynolds number $\mathrm{Re}$ is the ratio of the inertial to viscous forces in the fluid. and is an important parameter to match when working with different fluid properties and scaled geometries. For the liquid phase,

$$
\operatorname{Re}_{f}=\frac{\rho_{f} u_{f} D_{h y}}{\mu_{f}}
$$


Table 3.1 (continued)

*** Bubble size is believed to be an important parameter to match when simulating SG flow patterns. The $\sigma /\left(\rho_{r}-\rho_{g}\right)$ criterion shown in this table is from a typical expression for bubble size at departure during pool boiling [3]:

$$
d_{b}=C_{\mathrm{l}}\left[\frac{\sigma}{g\left(\rho_{f}-\rho_{g}\right)}\right]^{1 / 2}
$$

where $C_{1}$ is a function of the contact angle between the bubble and the surface. This criterion is a crude one, as bubbles tend to coalesce after departure, and forced convection (as opposed to pool boiling) produces finer bubbles. 


\section{Chapter 4}

\section{EXPERIMENTS IN AIR/WATER LOOPS}

\subsection{Rationale}

The first step in understanding flow patterns near supports was to conduct small-scale experiments with mock-ups of the SG components, under conditions that were inexpensive to produce and easy to control. Over a 3-year period between 1991 and 1994, ambient water tests were carried out on transparent acrylic test-sections. This was an opportunity to develop measuring techniques and to discover the important parameters that characterized the flow patterns.

\subsection{Description of Test Loop}

Tests were carried out in an air/water loop with half-rod, 7-rod, and 6-rod SG mock-ups containing the supports. [The very first test-section, a single-rod SG mock-up, was discarded as the flow area ratio between free-span and support regions was much greater than in the SG, giving rise to flow patterns that were subsequently determined to be unrealistic. This exercise showed the importance of representative flow area ratios.] Four types of CANDU SG supports were tested in the air/water loop (see Table 4.1 and Figure 4.1): the Bruce broached plate, Pickering A lattice bars, Darlington lattice bars, and Wolsong-1 formed bars.

Dimensions were scaled up by 20 to $50 \%$ for better observation and to accommodate instruments. The scaling factor for each mock-up was determined by comparing the testsection tube pitch with the appropriate SG tube pitch. As indicated in Table 4.1, the flow area ratio between the free-span and support regions, as well as the height-to-diameter ratio for the support channels, remained representative of the SG. 
Comparisons between mock-up supports (Figure 4.1) and actual SG supports (Figure 1.5) show that, geometry-wise, all mock-ups except those for the Darlington lattice bars were fully representative of the SG supports. As shown in Figure 1.5, the Darlington support is a complex one with every $8^{\text {th }}$ bar a tall (high) bar instead of a short (low) bar. For the mock-up, two intersecting high bars plus two adjacent low bar sections (one upper, one lower) were therefore chosen as a "worst case" from the standpoint of flow restriction.

The tests were conducted at room temperature and near-atmospheric pressure in a pumped water loop (Figure 4.2). Liquid velocities representing the lower region of the SG (0.5 to $1.5 \mathrm{~m} / \mathrm{s})$ were used. The combination of test fluid properties and scaled-up geometries gave liquid Reynolds numbers that were low by a factor of about 6 compared with the actual SG. This implied that the more dominant flow patterns would be observed in flow visualization tests, as smaller effects would be damped in the lower temperature, higher viscosity test fluid.

\subsection{Flow Visualization}

The objective of the flow visualization tests was to observe how each support affects the flow along the SG tubes. As discussed in Chapter 3, several flow visualization techniques were tried. The most useful technique involved introducing air bubbles into the flowing water as a flow visualization tool.

\subsubsection{Results for Clean Supports}

Figures 4.3a to 4.3d show drawings of each support (in the 6-rod SG mock-up) and its characteristic air/water flow pattern, at a liquid velocity of $\sim 1 \mathrm{~m} / \mathrm{s}$. Photographs were taken, but they could not show the dynamics of the flow; video recording were more helpful. 
In general, flow upstream of all the supports was uniform, except for "necking" of the flow within a few centimeters of the support. This necking was less obvious with the bartype supports than with the broached plate, which caused a sharp and relatively large contraction in flow area.

Downstream flow patterns were influenced to a greater extent by the supports. The broached plate (Figure 4.3a) caused dramatic flow recirculation, with eddies of swirling air bubbles extending 5 to $10 \mathrm{~cm}$ past the plate. The Darlington lattice bars (Figure 4.3b) featured recirculation over the high-bar intersection. The low-bar arrangement caused visible flow redirection: air films wrapped around the bars and stable air pockets formed above them, indicating low-velocity regions. The Pickering lattice bars (Figure 4.3c) also featured recirculation or air stagnation above the bars, but to a lesser extent. Flow patterns with the Wolsong formed bars (Figure 4.3d) were very uniform, with only thin, stable air pockets above the bars.

The air/water flow patterns with the broached plate were very similar to the steam/water ones described in a 1983 EPRI report [1] (see Section 2.2), implying that the dominant patterns are the same regardless of the fluid used. Also, the flow patterns were generally the same regardless of whether the half-rod, the 7-rod, or the 6-rod SG mock-ups was used. The only significant exception was that the downstream recirculation pattem in the multiple-rod mock-ups had a 3-dimensional structure, and was therefore more complex than in the half-rod mock-up.

\subsubsection{Results for Partially-Blocked Flow Channels}

Using bubbly air/water mixtures, experiments were carried out in which holes on one side of a broached plate mock-up were covered on the downstream side with putty, simulating blockages in those holes. With this partial blockage, the air/water flow was fast and jetlike on the clean side, and slow and recirculating on the blocked side. The more open 
channel was characterized by a higher velocity and larger quantity of air bubbles, due to the need to maintain equal pressure loss across the two parallel channels (which is effectively the maintaining of mass and momentum balances). A schematic of this flow pattern is shown in Figure 4.4.

\subsubsection{Results using Metallic Flakes}

A flow visualization technique was tried in which small ( 0.5 to $1 \mathrm{~mm}$ in diameter), discshaped, easily-suspended aluminum flakes were introduced into the test loop. As a technique it was unsuccessful, because the flakes were difficult to track, but an unexpected observation was made: the flakes tended to collect on the upstream lands* of the broached plate, where flow branched into adjacent broach holes (Figure 4.5a). They accumulated to a point of equilibrium volume, which depended on the flow rate, beyond which additional flakes were sheared off by the flow. Very little accumulation was found downstream (Figure 4.5b), possibly because flow recirculation swept the plate clean. The flakes were held by dynamic forces: as soon as the flow was stopped, the flakes fell to the bottom of the test-section.

None of the bar designs showed accumulation to the same degree as the broached plate. With the bars, instead of being trapped on horizontal surfaces, flakes became wedged in the vertical crevice between a tube and support bar.

\subsection{Pressure Loss}

The objectives of the pressure-loss tests were: (a) to obtain an overall flow resistance coefficient ( $\mathrm{K}$-factor) for each support by measuring total pressure loss across the support, and (b) to find regions of local pressure extremes by measuring axial pressure profiles. The tests were carried out in single-phase water. 


\subsubsection{Experimental Method}

As shown in Figure 4.6, both sets of measurements were taken using a thin hollow sliding probe with a static pressure tap near one end, which could be inserted through the support and pulled back at measured distances to give axial pressure profiles. [The technique of the sliding probe was developed in earlier work [2].] At each axial location, the voltage signals from the differential pressure transducer (DP cell) were time-averaged, to reduce the effect of signal noise. Voltages were converted to pressure differences following calibration equations obtained prior to testing.

The DP cell sensed pressure differences between the fixed reference tap and the sliding tap. As profiles could take $\sim 1 / 2$ hour to obtain, such relative pressures were more precise than absolute values, because they were not affected by fluctuations of overall loop pressure with time. Also, in the unheated single-phase flow, the DP cell does not measure any pressure loss due to the static head between the vertical taps, which can otherwise swamp other pressure losses.

\subsubsection{Axial Pressure Profiles}

A set of measured axial pressure profiles is shown in Figures 4.7a to 4.7d. Both the freespan velocity $(1.24 \mathrm{~m} / \mathrm{s}$ ) and the probe radial location (in the subchannel closest to the center rod) are the same in these figures. The difference between the upstream and downstream plateau values is taken as the total pressure loss. These experimental total losses, converted to resistance coefficients or $\mathrm{K}$-factors (and labelled $\mathrm{K}_{\text {expt }}$ ), are listed in Table 4.2.

The broached plate caused the greatest overall pressure loss, and the Wolsong bars caused the least. The Darlington mock-up gave a greater loss than the Pickering one, which is consistent with the visually observed flow patterns that are more turbulent near the 
Darlington support. [The comparison is somewhat unfair because the Darlington mockup is a "worst case" geometry: had the geometry been more representative, the two types of lattice bars would have probably had similar K-factors.]

The broached plate pressure profile shows a minimum just downstream of the broach hole inlet, which corresponds to the location of the greatest expected flow contraction. A partial pressure recovery occurs relatively slowly as flow continues to re-develop well past the support. The Darlington pressure profile shows two sharp pressure drops, corresponding to the sudden contraction at the first (lower) and second (upper) low bars. Pressure recoveries past these bars are also relatively slow. The Pickering profile also indicates where flow contractions occur within the support. The Wolsong pressure profile features a small drop at the support entrance, a further slight decrease due to friction along the support, then a quick recovery past the support.

\subsubsection{Calculated K-factors}

The K-factors were also calculated using a simple contraction-friction-expansion model. [A more complex two-phase version of this model is used in the TSFOUL program, as described in Section 7.3.5.] The calculated overall $\mathrm{K}$-factors (labelled $\mathbf{K}_{\text {calc }}$ ) were obtained as follows (using the nomenclature listed at the start of the thesis) $[3,4]$ :

$$
\begin{aligned}
& K_{\text {con }}=0.5\left(1-\frac{A_{\text {sup }}}{A_{f s}}\right) \\
& K_{f n c}=f \frac{L_{\text {sup }}}{D_{h y}}
\end{aligned}
$$

where $f=\left\{-1.8 \log _{10}\left[\frac{R e_{f}}{6.9}+\left(\frac{\varepsilon / D_{h y}}{3.7}\right)^{1.11}\right]\right\}^{-2}$ 


$$
K_{\exp }=\left(1-\frac{A_{\text {sup }}}{A_{f s}}\right)^{2}
$$

The $\mathrm{K}_{\text {calc }}$ values are listed in Table 4.2, and are reduced to their contraction, friction, and expansion components in Table 4.3. For comparison with experimental K-factors, friction factors for both smooth pipes $\left(\varepsilon / D_{\text {hy }}<0.00005\right)$ and rough pipes $\left(\varepsilon / D_{\text {hy }}=0.016\right.$, which corresponds to a plausible roughness height $\varepsilon$ of $0.1 \mathrm{~mm}$ ) were used. The simplified geometries assumed for the calculations are shown below Table 4.3.

In all cases, the calculated $\mathrm{K}$-factor is less than the experimental one; possible reasons for this are:

- the model relies on simple "step" geometries, whereas the true geometries are threedimensional and complex,

- flow redirection is not taken into account (which may explain in part the large discrepancy between measured and calculated K-factors for the Darlington bars, for which cross-flow was observed between the upstream and downstream low bars), and

- flow is assumed to be fully developed for the calculated K-factors, whereas the supports are typically within the flow development length and consequently have higher friction factors than calculated.

The experimental K-factors apply only to clean supports (as opposed to fouled). Also, although flow area ratios were made to be representative of the SG, the test-section and mock-ups were scaled up for the experiments and include artificial walls.

\subsubsection{Effect of Subchannel}

For all designs except the broached plate, the shape of the axial profile between the plateaus depended on the local contractions and expansions in the particular subchannel 
containing the probe. An example is shown in Figures 4.8a and 4.8b for the Darlington mock-up, where the first profile was obtained in the high-bar region and the second in the low-bar region. [The profile in Figure 4.7b was obtained with the probe bounded by a high bar and a low bar.] While the overall loss is the same, the high-bar profile does not show pressure losses and recoveries associated with a changing flow area within the support. The behaviour illustrated in Figures $4.7 \mathrm{~b}, \mathbf{4 . 8 \mathrm { a }}$, and $\mathbf{4 . 8 \mathrm { b }}$ also reflects cross-flow effects. The good agreement in overall pressure loss in these three figures gives confidence in the measured values for $K$.

\subsubsection{Effect of Edge Sharpness}

The mock-ups tested in the experiments all had $90^{\circ}$ sharp edges. However, blueprints for the Pickering and Darlington lattice bars specify to "remove sharp edge" on the four comers of each bar, or specify "full rad" and "break comers acceptable". To investigate the effect of rounded edges, the edges of the bars on the Darlington mock-up were filed by hand.

Figure 4.9 shows that, for the same velocity and probe location, the measured pressure profile is smoother and the overall pressure loss considerably lower (by $27 \%$ ) with the rounded edges.

Furthermore, the component of pressure loss most affected by the edge rounding is the contraction of flow into the support. Subsequent to all the contractions through the mock-up lattice (i.e., at the $8 \mathrm{~cm}$ mark in Figure 4.9), the profiles become similar, indicating little difference in the friction or expansion components. This suggests that a significant reduction in pressure loss could be achieved by rounding only the leading edges of the support. 


\subsection{Velocity and Turbulence}

The objective of the velocity measurements was to find regions of stagnation or low flow. This, in turn, would indicate where particulates could settle on the support. A secondary objective was to explore the velocity measurement technique.

\subsubsection{Experimental Method}

The measurements were carried out at a single-phase free-span velocity of about $1.4 \mathrm{~m} / \mathrm{s}$. A non-intrusive laser doppler velocimetry (LDV) technique was used to generate maps of single-phase local velocity and turbulence intensity. For this technique, a single laser beam is divided by optical lenses into two beams that intersect in the flow, as shown in Figure 4.10. The two intersecting laser beams create an interference or fringe pattern. Light scattered from small particles moving with the flow through the fringe pattern is detected by the optical system. The scattered light is of another frequency due to the movement of the particles; this is the Doppler effect. The time difference between peaks in the scattered light is solely determined by the velocity of the particle and the fringe spacing, a parameter determined by the optical set-up. The LDV signal processor then converts the frequency shift to a flow velocity.

The variation in the measured velocities typically varied about the mean with a Gaussian distribution, whose standard deviation was used to calculate the turbulence intensity. An example of the data from a single velocity measurement is shown in Figure 4.11.

The LDV system could measure velocity or turbulence intensity in a single direction only. However, a two-dimensional velocity vector map could be constructed by rotating the probe, thereby determining the angle of greatest detector response. 


\subsubsection{Results}

Of the many measurements taken with different test-sections, the set shown in Figure 4.12 best illustrates the velocity distribution. These data were taken with the half-rod test-section which had visual access to the inside of a broach hole. Vectors on this twodimensional velocity map were determined by rotating the LDV probe.

As observed during flow visualization (Section 4.1), the flow accelerated as it entered the broached plate, and jetted out at the exit, leaving a small zone of low velocity under the support and a larger zone above the support. A few mm into the broach hole, the turbulence intensity appeared to be high near the edge of the plate; this was probably not true turbulence but a steep velocity gradient that the beams needed to straddle. This is consistent with the expected presence of a vena contracta* at this location.

Velocity measurements with the three other support designs (Figures 4.13a to 4.13c) were made with the 6-rod test-section, and are not as detailed. While the 6-rod test-section was more representative of the actual SG flow environment, the subchannels surrounding the center tube could not be accessed easily by the laser beams. The measurements were limited to regions that could be viewed from outside the test-section.

In Figures $4.13 \mathrm{a}$ to $4.13 \mathrm{c}$, low velocity, when coupled with high turbulence intensity, indicates a significant cross-flow component. High velocity coupled with low turbulence intensity indicates strong axial flow.

For all the designs, the flow upstream of the support appears to be uniform; the support begins to affect the flow at only a short distance from the support. These findings are consistent with visual observations of parallel flow changing to flow "necking" immediately before the support. 
Downstream, all the turbulence maps show darker regions (indicating higher turbulence) immediately above the support bars. These coincide with the regions of flow recirculation observed visually. The disruptive effect of the bars is much more pronounced with the Pickering and Darlington lattice bars than with the Wolsong formed bars; again, this agrees with observations and is likely related to relative bar thicknesses and arrangement. The Darlington velocity maps show a less uniform distribution radially than the Pickering maps, probably due to the strong cross-flow observed between the Darlington low bars.

\subsection{Air/Water Void Fraction}

The objective of the air/water void fraction* tests was to confirm observed flow patterns, and to identify regions near a SG support that could be deficient in either the liquid or the gas phase (i.e., determine the distribution of the gas and liquid phases). A secondary objective was to explore the void fraction measurement technique.

The tests were carried out using the 7-rod test-section with the broached plate support. The air and water flow rates and average void fraction were chosen to give an easilydetermined void distribution; resulting low-velocity bubbly flow was probably characteristic of the lower SG regions.

\subsubsection{Experimental Method}

The test apparatus is shown in Figure 4.14. The technique is similar to that of LDV, except that only one laser beam is used. The optical fiber carrying the beam was threaded through the center tube such that only its tip protruded into the flow. The laser light beam travelled down the fiber into the fluid; scattered light also travelled back up the fiber to a photo-detector. The intensity of scattered light increased measurably when the fiber tip 
encountered a gas bubble instead of liquid. The fiber tip was made as unobtrusive as possible so the bubbles would not be diverted around it.

A threshold level of scattered light was established with the fiber tip in liquid only. An electronic processor then determined void fraction (in \%) by the fraction of time during a pre-set time period (e.g., 30 seconds) that this threshold was exceeded because the tip had encountered the gas phase.

\subsubsection{Results}

A two-dimensional void fraction map (Figure 4.15) was generated by rotating and raising the tube on which the fiber was mounted. The fiber protruded $5 \mathrm{~mm}$ into the flow, so that the tip described a circle half-way between the tubes, as shown in Figure 4.15. The center tube was chosen to house the fiber, as the flow patterns surrounding this tube would then be unaffected by proximity to the wall of the flow pipe.

The map in Figure 4.15 shows small pockets of low void near the corners of the broach holes (at $20^{\circ}, 90^{\circ}$, and $140^{\circ}$ ). Pockets of high void can be found 0.5 to $1.0 \mathrm{~cm}$ above the web of the plate (near $0^{\circ}$ and $120^{\circ}$ ). This is consistent with visual observation of airentraining eddies or regions of recirculation directly above the broached plate, as described in Section 4.3.1.

The next Chapter describes the experiments undertaken with Freon-11 as modeling fluid. 


\section{References}

[1] D.S. Cassell and D.W. Vroom, "Thermalhydraulic Tests of SG Tube Support Plate Crevices", EPRI-NP-2838 (Volumes 1-3), 1983 January.

[2] H.E.C. Rummens, G.R. Dimmick and P.E. Bindner, "Measurement of Axial Pressure Profiles for Nuclear Fuel Assemblies by using a Sliding Probe", Journal of Experimental Thermal and Fluid Science, Vol.14, pp.213-223 (1997).

[3] Crane Handbook: Flow of Fluids through Valves, Fittings, and Pipes, Crane Co., 1980, p.3-4.

[4] S.E. Haaland, "Simple and Explicit Formulas for the Friction Factor in Turbulent Pipe Flow", Journal of Fluids Eng., 1983 March, pp.89-90. 
Table 4.1: Dimensions of mockups for air/water and comparison with actual

\section{BRUCE BROACHED PLATE}

tube pitch [m]

tube diameter $[\mathrm{m}]$

broached plate height [m]

support flow area per tube ${ }^{1}\left[\mathrm{~m}^{2}\right]$

support wet perimeter per tube [m]

support equivalent diameter ${ }^{2}[\mathrm{~m}]$

flow area ratio ${ }^{3}$

porosity ${ }^{4}$

height to diameter ratio

liquid $\operatorname{Re}$ within support $[-]^{5}$

PICKERING A LATTICE BARS

tube pitch [m]

tube diameter [m]

lattice bar thickness [m]

lattice bar height (total) [m]

support flow area per tube $\left[\mathrm{m}^{2}\right]$

support wet perimeter per tube [m]

support equivalent diameter [m]

flow area ratio

porosity

height to diameter ratio

liquid $\operatorname{Re}$ within support [-]
Bruce A\&B

0.0203

0.0130

0.0254

0.0000966

0.0891

0.00434

$2.39: 1$

0.42

5.85

$3.3 \times 10^{4}$

Pickering A

0.0191

0.0127

0.00300

0.0508

0.000132

0.0781

0.00676

$1.43: 1$

0.70

7.51

$5.1 \times 10^{4}$
0.0310

0.0191

0.030

0.000217

0.128

0.00677

$2.51: 1$

0.40

4.43

$0.74 \times 10^{4}$

0.22

1.52

1.47

1.2

scale factor

.52
.47
.2

scale factor

1.62

1.50

1.6

0.0047

0.076

0.000402

0.201

0.00799

$1.36: 1$

0.74

9.51

$0.87 \times 10^{4}$

0.17

continued

1 measured at mid-point of support

2 equiv diameter $=4 \times$ (flow area per tube) / (wet perimeter per tube)

3 flow-area ratio = (approach region flow area) $/$ (support region flow area)

4 porosity $=1 /($ flow-area ratio)

5 determined for $u_{\mathrm{f}}=1.0 \mathrm{~m} / \mathrm{s}$, with fluid properties $\rho_{\mathrm{f}}$ and $\mu_{\mathrm{f}}$ taken from Table 3.1 , columns 2 and 5 , and equiv diameter as in footnote 2

6 assumes lattice consists of only high bars

7 assumes lattice consists of only low bars; dimensions are taken at mid-point of one set of low bars

8 determined at mock-up mid-point where only high bars are present (resembles lowbar-only geometry marked with Footnote 7 
Table 4.1 (continued)

\author{
DARLINGTON LATTICE BARS \\ tube pitch [m] \\ tube diameter [m] \\ lattice bar thickness [m] \\ lattice bar height (high) / low [m] \\ region of applicability \\ support flow area per tube $\left[\mathrm{m}^{2}\right]$ \\ support wet perimeter per tube [m] \\ support equivalent diameter [m] \\ flow area ratio \\ porosity \\ height to diameter ratio \\ liquid $\operatorname{Re}$ within support [-]
}

\section{WOLSONG-1 FORMED BARS}

tube pitch [m]

tube diameter $[\mathrm{m}]$

formed bar thickness [m]

formed bar height [m]

support flow area per tube $\left[\mathrm{m}^{2}\right]$

support wet perimeter per tube [m]

support equivalent diameter [m]

flow area ratio

porosity

height to diameter ratio

liquid $\operatorname{Re}$ within support [-]
Wolsong 2-4 \&

Darlington

mock-up scale factor

$\begin{array}{lcl}0.0245 & 0.0310 & 1.29 \\ 0.0159 & 0.0191 & 1.20 \\ 0.00439 & 0.0055 & 1.2 \\ 0.080 / 0.025 & 0.099 / 0.031 & 1.2\end{array}$

high bars $^{6}$ low bars ${ }^{7}$ middle $^{8}$

$\begin{array}{lll}0.000121 & 0.000199 & 0.000395\end{array}$

$\begin{array}{lll}0.134 & 0.0982 & 0.193\end{array}$

$\begin{array}{lll}0.00362 & 0.00811 & 0.00819\end{array}$

$2.52: 1 \quad 1.53: 1 \quad 1.38: 1$

$\begin{array}{lll}0.40 & 0.65 & 0.72\end{array}$

$\begin{array}{lll}22.1 & 3.08 & 12.1\end{array}$

$6.1 \times 10^{4} \quad 0.89 \times 10^{4} \quad 0.15$

Wolsong-1

mock-up scale factor

$\begin{array}{ll}0.0310 & 1.54\end{array}$

0.0159

0.0191

1.20

0.00157

0.0020

1.3

0.0508

0.000207

0.076

1.5

0.118

0.000415

0.00702

0.227

$1.41: 1$

0.00731

0.71

$1.31: 1$

7.24

0.76

10.40

$5.3 \times 10^{4}$
$0.80 \times 10^{4}$

0.15

\section{NOTE:}

All mock-ups except the Darlington lattice bars are fully representative of the SG supports. The Darlington mock-up consists of two intersecting high bars plus two adjacent low-bar sections (one upper, one lower); this configuration was chosen as a "worst case". 
Table 4.2: Support K-factors, measured and calculated

[Velocities and K-factors are based on the free-span flow area.]

$$
K_{\text {exp }}=\frac{2 \Delta P_{\text {tot }}}{\rho u^{2}}
$$

run \# $\Delta \mathbf{P}_{\text {tot }}$ density velocity $\mathbf{K}_{\text {expt }} \mathbf{K}_{\text {eale }}$ diff $\mathbf{K}_{\text {eale }}$ difi (kPa) $\left(\mathrm{kg} / \mathrm{m}^{3}\right) \quad(\mathrm{m} / \mathrm{s}) \quad\left(\right.$ smooth surf.) $\quad\left(\varepsilon / \mathrm{D}_{\mathrm{hy}}=0.016\right)$

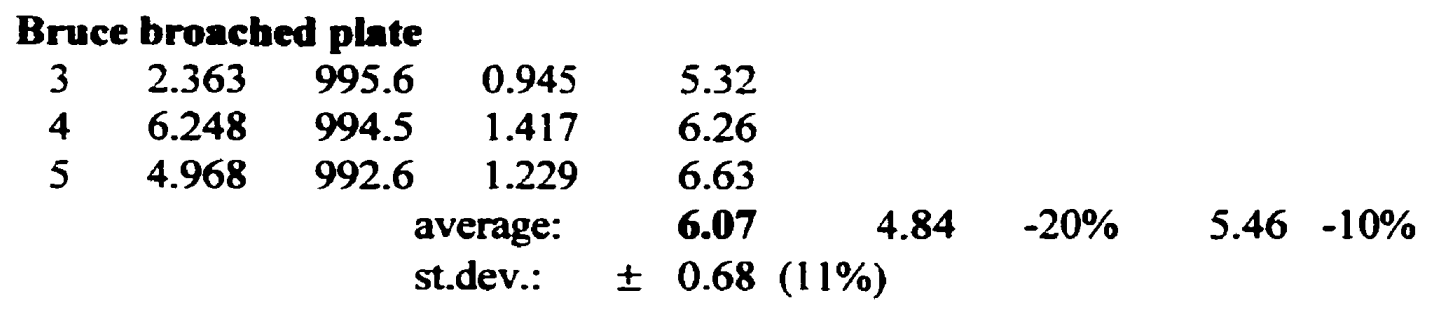

\section{Pickering A lattice bars}

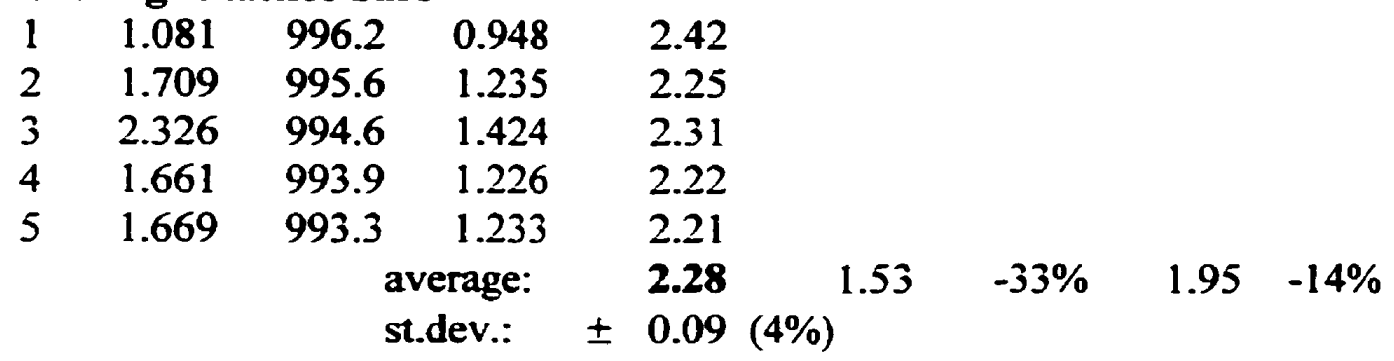

Darlington Iattice bars

\begin{tabular}{|c|c|c|c|c|c|c|c|}
\hline 1.482 & 996.2 & 0.915 & 3.55 & & & & \\
\hline 3.463 & 994.8 & 1.244 & 4.50 & & & & \\
\hline 4.126 & 994.3 & 1.363 & 4.47 & & & & \\
\hline 3.325 & 993.6 & 1.243 & 4.41 & & & & \\
\hline 3.187 & 993.6 & 1.234 & 4.21 & & & & \\
\hline \multicolumn{3}{|c|}{ average: } & 4.23 & 2.49 & $-41 \%$ & 3.15 & $-25 \%$ \\
\hline \multicolumn{3}{|c|}{ st.dev.: } & 0.40 & & & & \\
\hline
\end{tabular}

\begin{tabular}{|c|c|c|c|c|c|c|c|c|}
\hline \multicolumn{9}{|c|}{ Wolsong-1 formed bars } \\
\hline 1 & 0.552 & 996.2 & 0.929 & 1.28 & & & & \\
\hline 2 & 1.001 & 995.6 & 1.239 & 1.31 & & & & \\
\hline 3 & 1.082 & 995.3 & 1.427 & 1.07 & & & & \\
\hline 4 & 0.918 & 993.0 & 1.236 & 1.21 & & & & \\
\hline 5 & 0.906 & 991.6 & 1.232 & 1.20 & & & & \\
\hline & & & erage: & 1.22 & 0.78 & $-36 \%$ & 1.14 & $-7 \%$ \\
\hline & & & lev.: & 0.09 & & & & \\
\hline
\end{tabular}


Table 4.3: Contraction, friction, and expansion components in calculated $\mathrm{K}$-factors

$$
\mathrm{K}_{\text {con }} \text { (fraction) } \mathbf{K}_{\text {fric }} \text { (fraction) } \mathrm{K}_{\mathrm{exp}} \text { (fraction) }
$$

Bruce broached plate

- smooth surfaces

$-\varepsilon / D_{\text {hy }}=0.016$

$1.90(39 \%) \quad 0.66(14 \%) \quad 2.28 \quad(47 \%)$

$1.90 \quad(35 \%) \quad 1.28 \quad(24 \%) \quad 2.28 \quad(42 \%)$

Pickering A lattice bars

- smooth surfaces

$-\varepsilon / D_{\text {hy }}=0.016$

$0.63(41 \%) \quad 0.64(42 \%) \quad 0.26(17 \%)$

$0.63(32 \%) \quad 1.06(54 \%) \quad 0.26(13 \%)$

Darlington lattice bars

- smooth surfaces

$0.99(40 \%)$

$0.79(32 \%)$

$0.71(28 \%)$

$-\varepsilon / D_{\text {hy }}=0.016$

0.99 (31\%) $1.46 \quad(46 \%)$

$0.71(22 \%)$

Wolsong-1 formed bars

- smooth surfaces

$0.20 \quad(26 \%) \quad 0.49 \quad(62 \%) \quad 0.10 \quad(12 \%)$

$-\varepsilon / D_{\text {hy }}=0.016$

$0.20(18 \%) \quad 0.84 \quad(74 \%)$

$0.10(8 \%)$

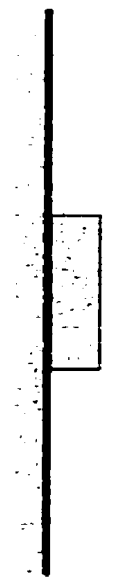

(a) Bruce broached plate

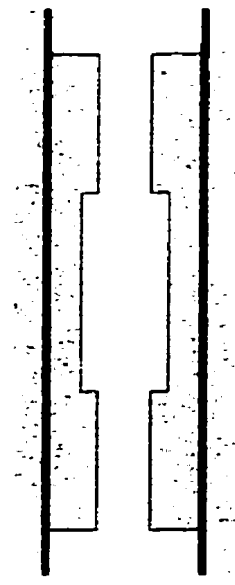

(c) Darlington lattice bars

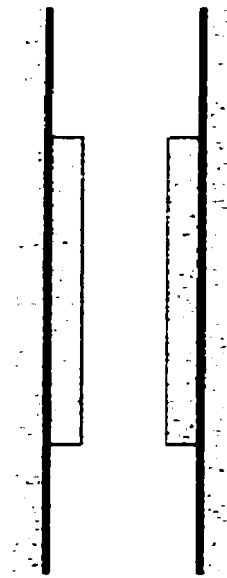

(d) Wolsong-1 formed bars 
Figure 4.1: Mockups tested in air/water

LOWER RIGHT: Bruce broached plate (scale-up factor 1.5)

LOWER LEFT: $\quad$ Pickering-A lattice bars (factor 1.6)

UPPER RIGHT: Wolsong-1 formed bars (factor 1.5)

UPPER LEFT: $\quad$ Darlington lattice bars (factor 1.3)

NOTE: Darlington bars should be rotated 90 degrees to match orientation of other mock-ups.

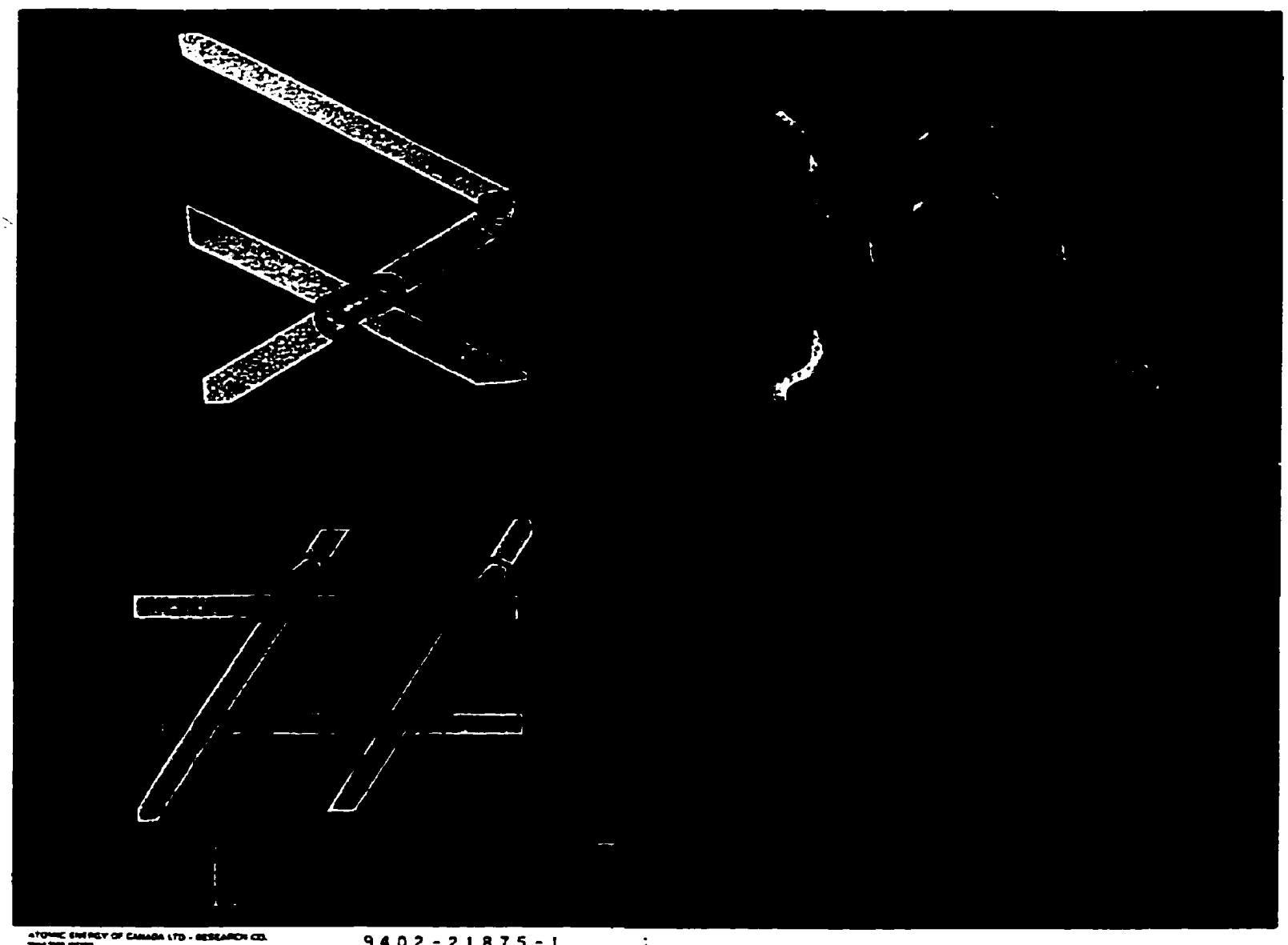


Figure 4.2: Air/water test loop

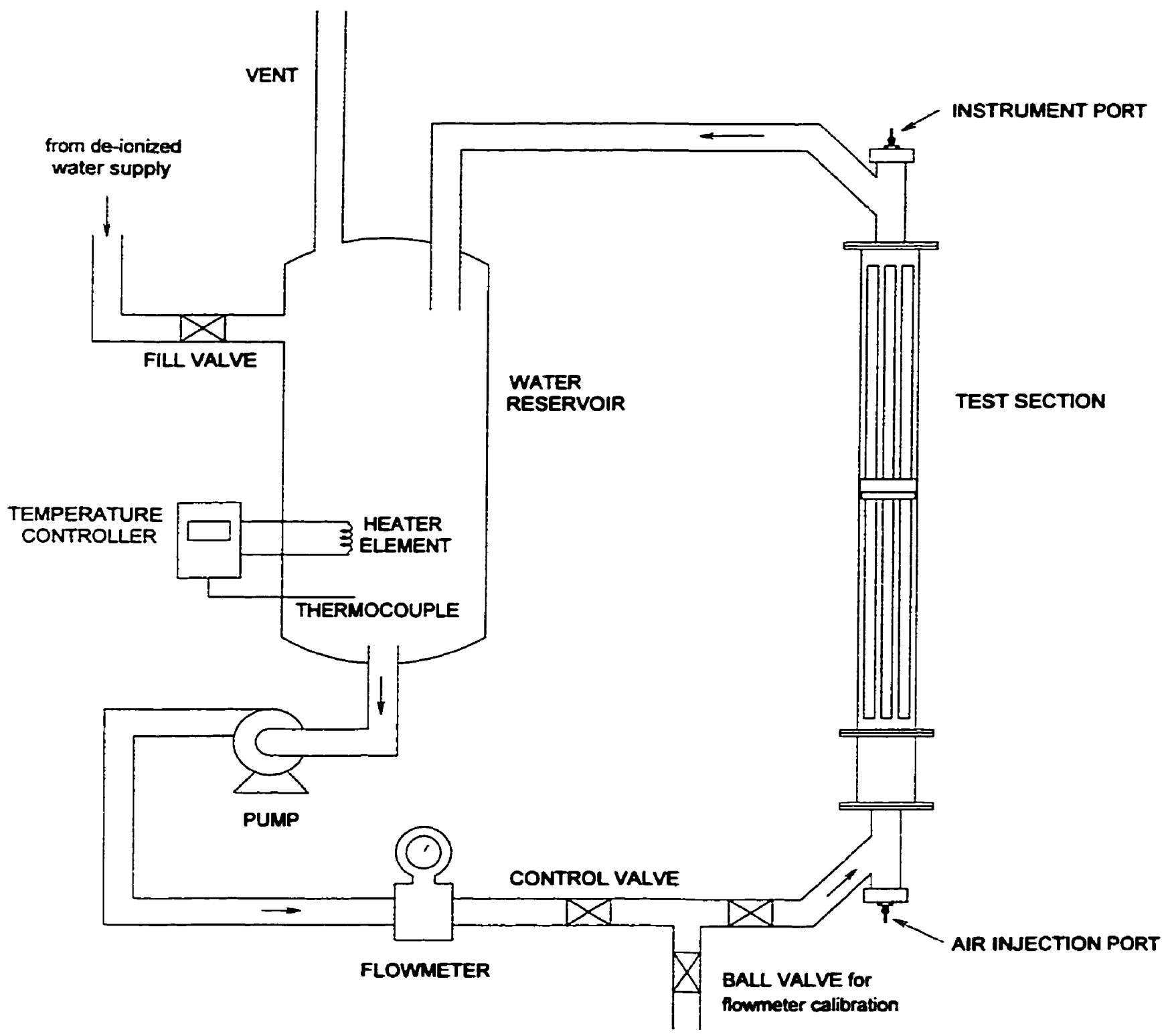


Figure 4.3: Observed flow patterns - clean supports

(a) Bruce broached plate

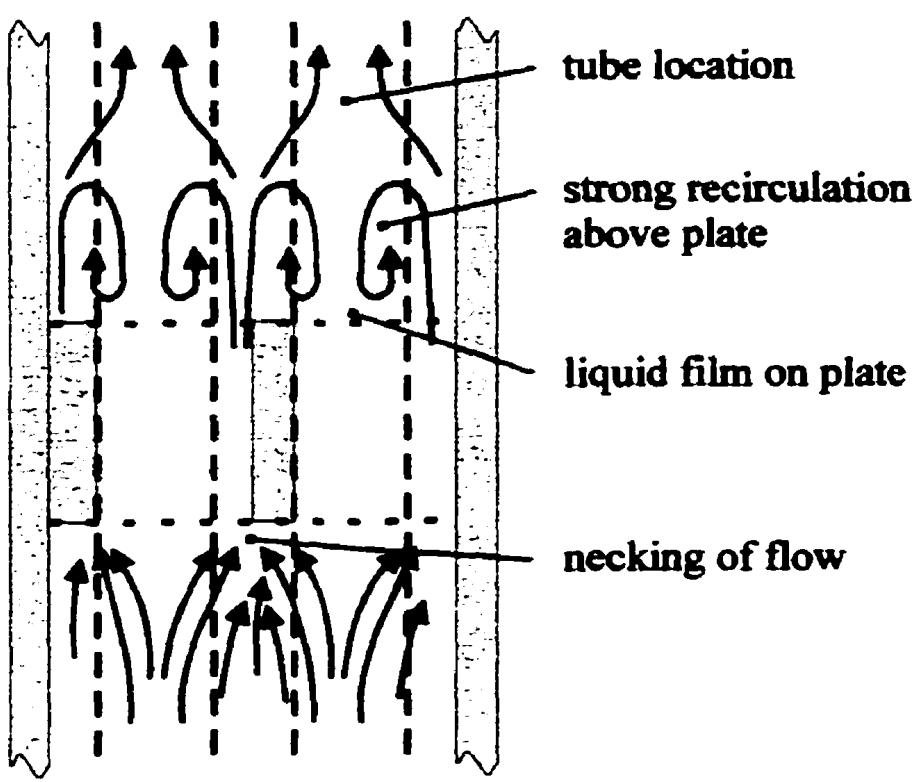

(b) Darlington lattice bars

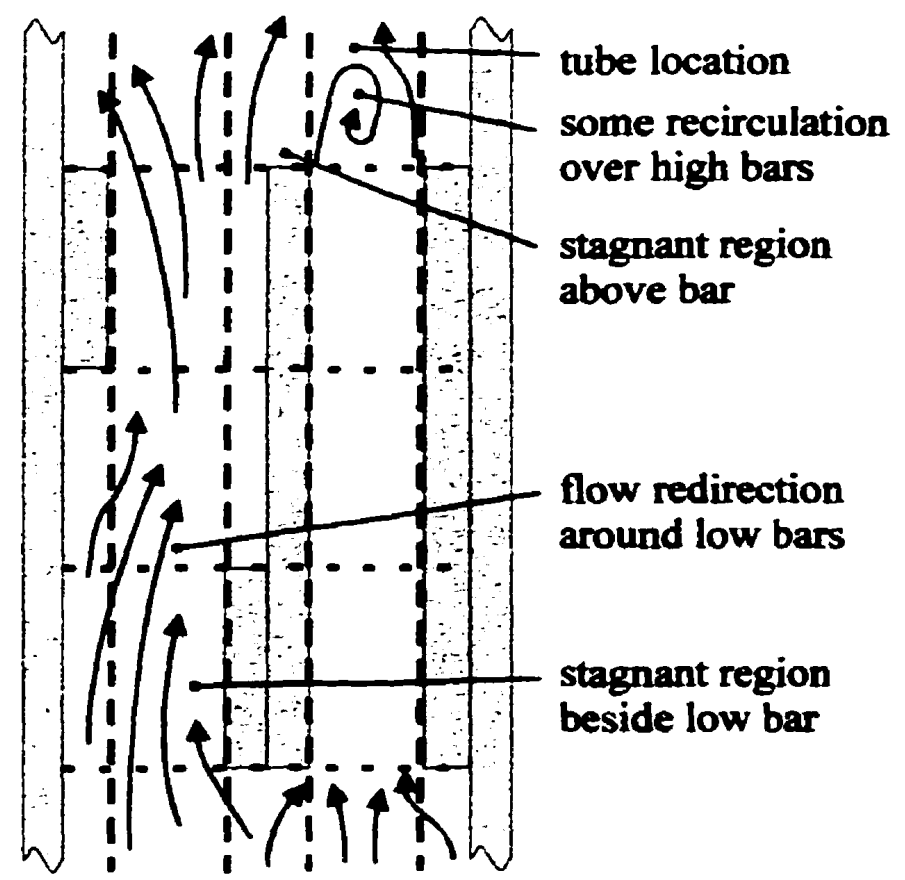


Figure 4.3 (continued)

(c) Pickering lattice bars

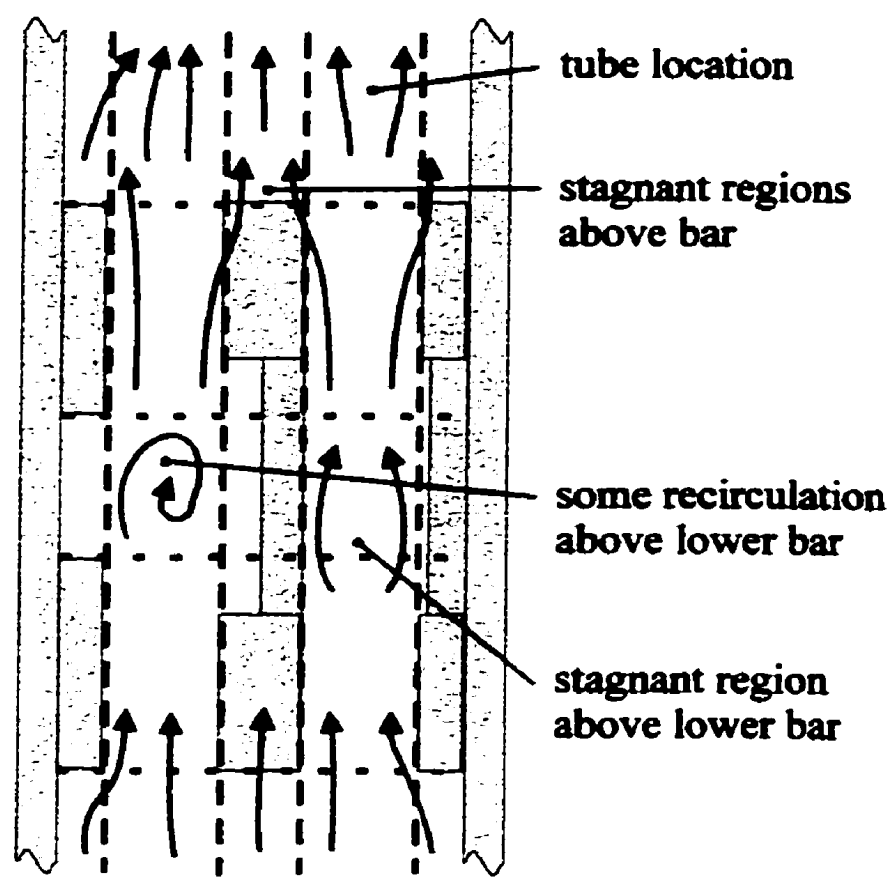

(d) Wolsong-1 formed bars

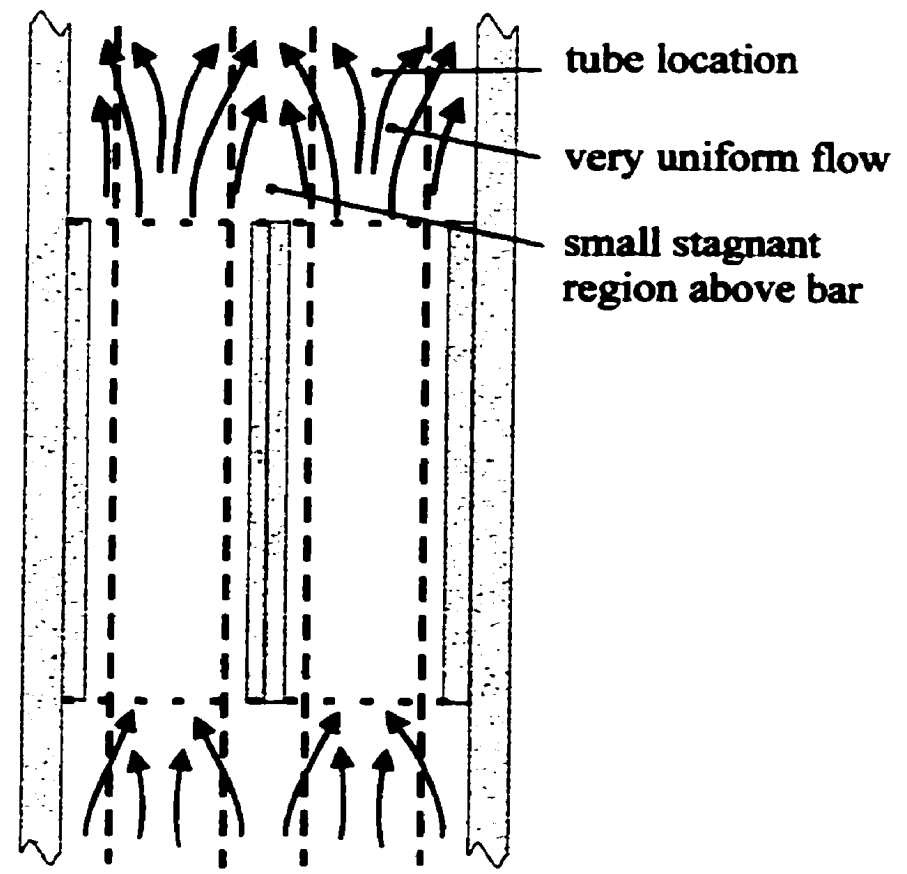


Figure 4.4: Observed flow patterns - partially-blocked channels

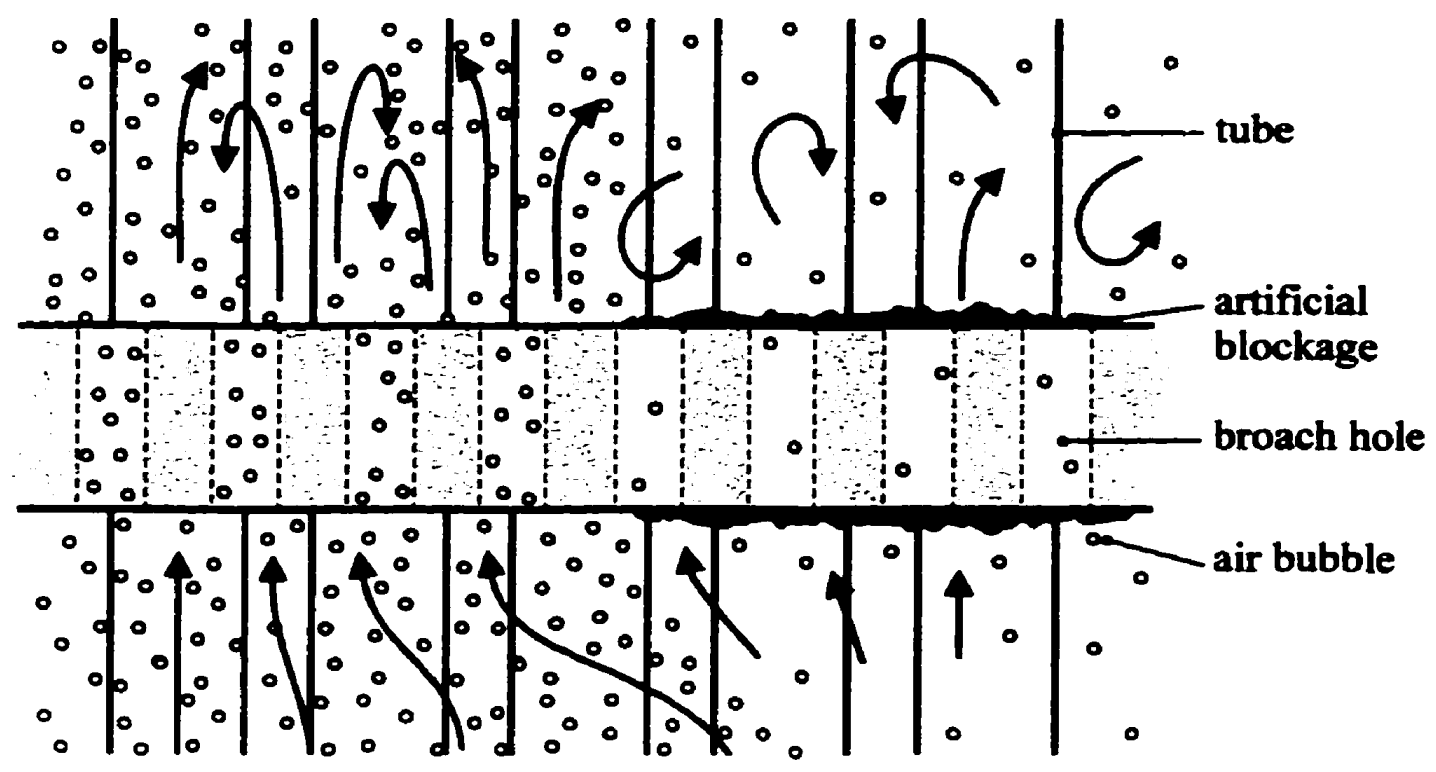


Figure 4.5: Observed flow patterns - deposition of metallic flakes

(a) upstream side

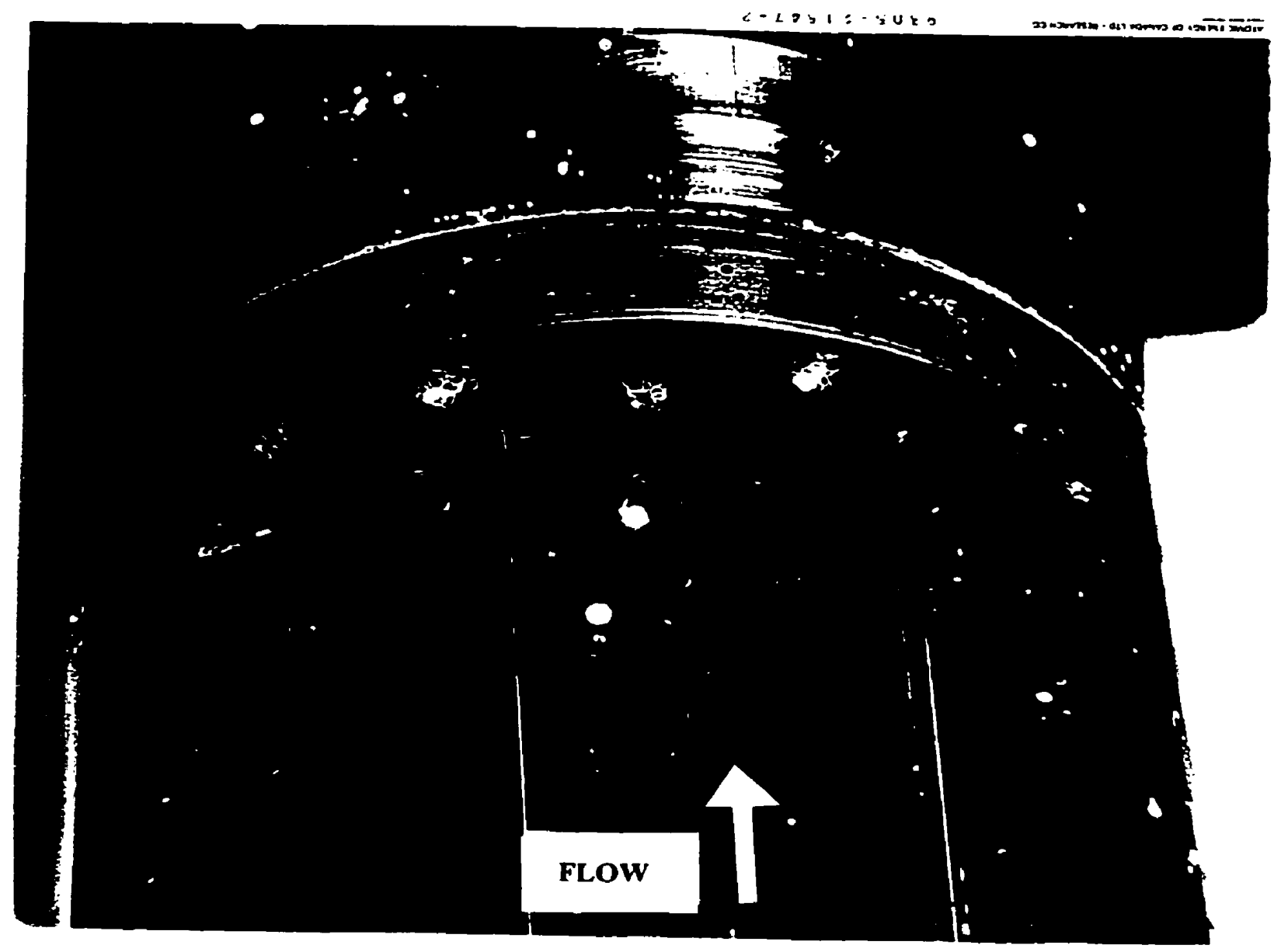


Figure 4.5 (continued)

(b) downstream side

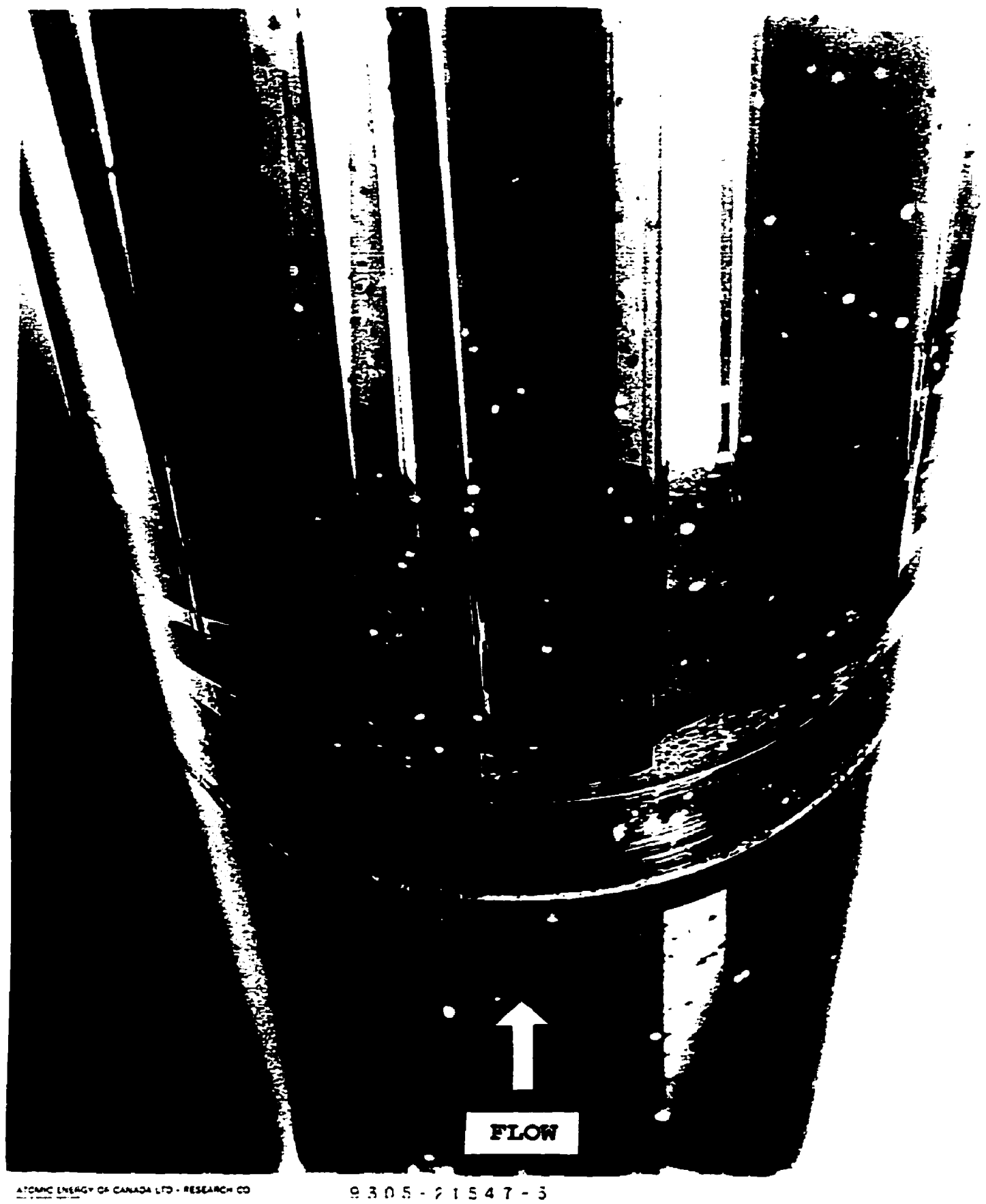


Figure 4.6: Pressure loss apparatus

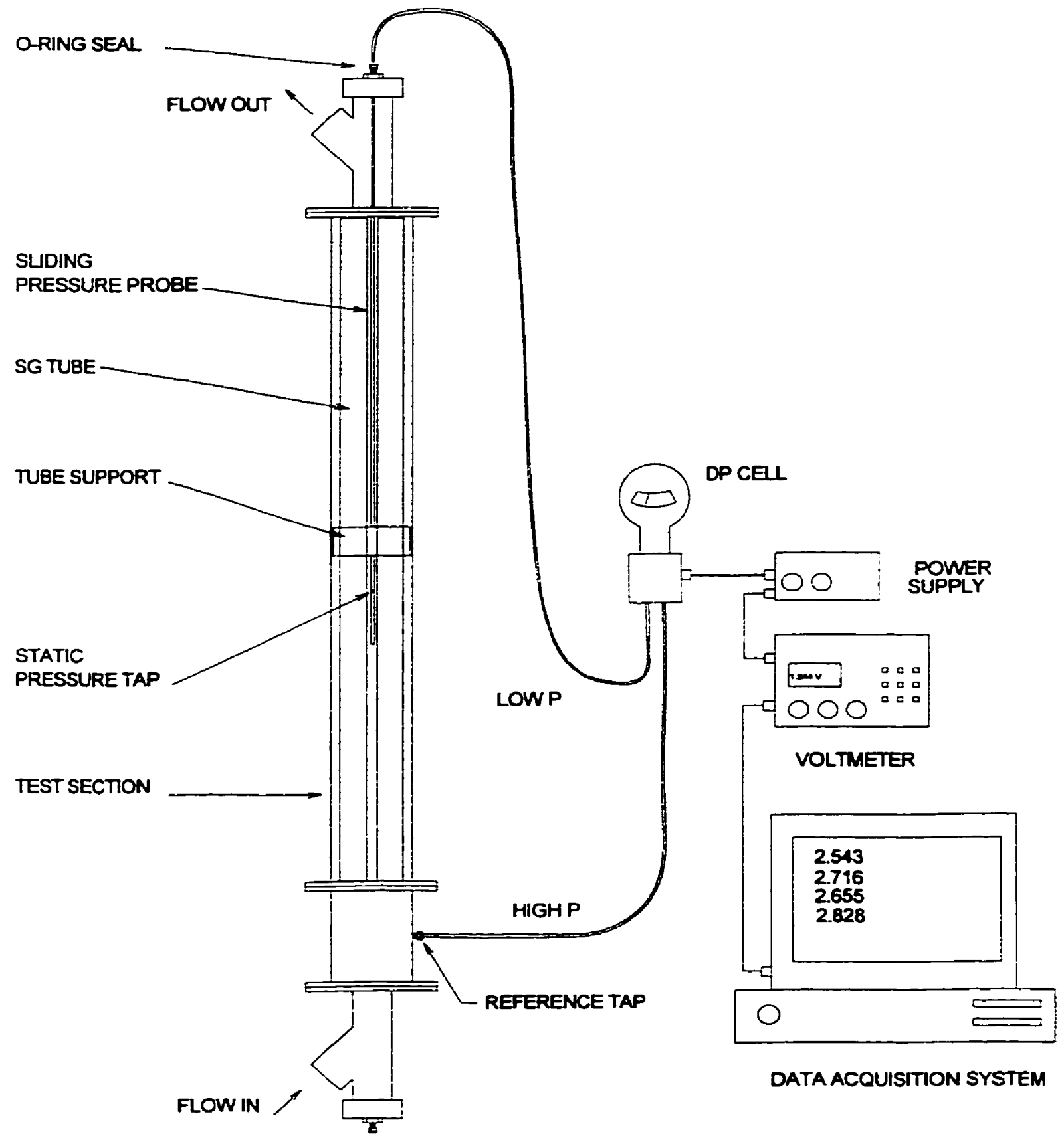


Figure 4.7: Pressure loss profiles for various supports

(a) Bruce broached plate
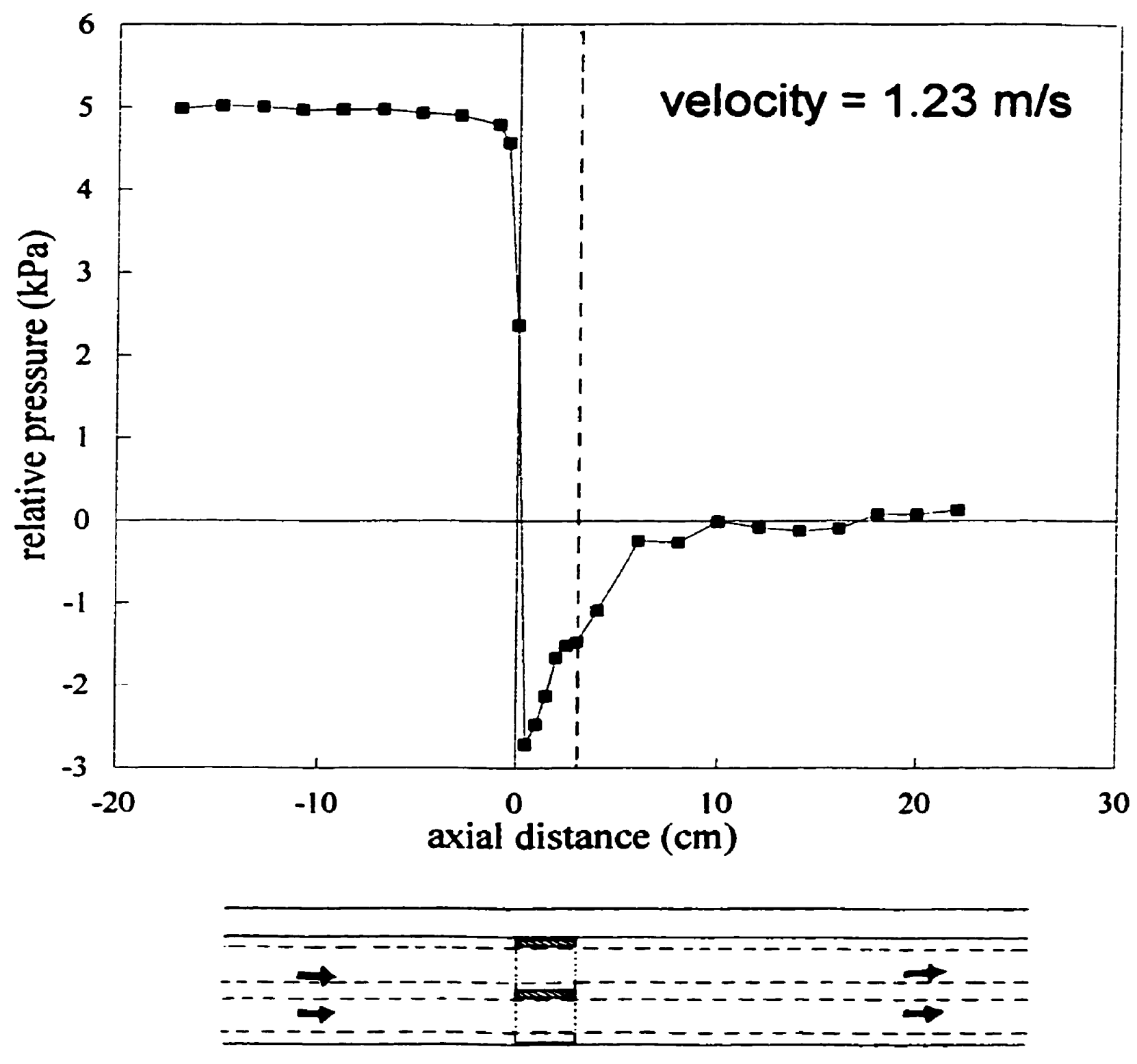
Figure 4.7 (continued)

(b) Darlington lattice bars
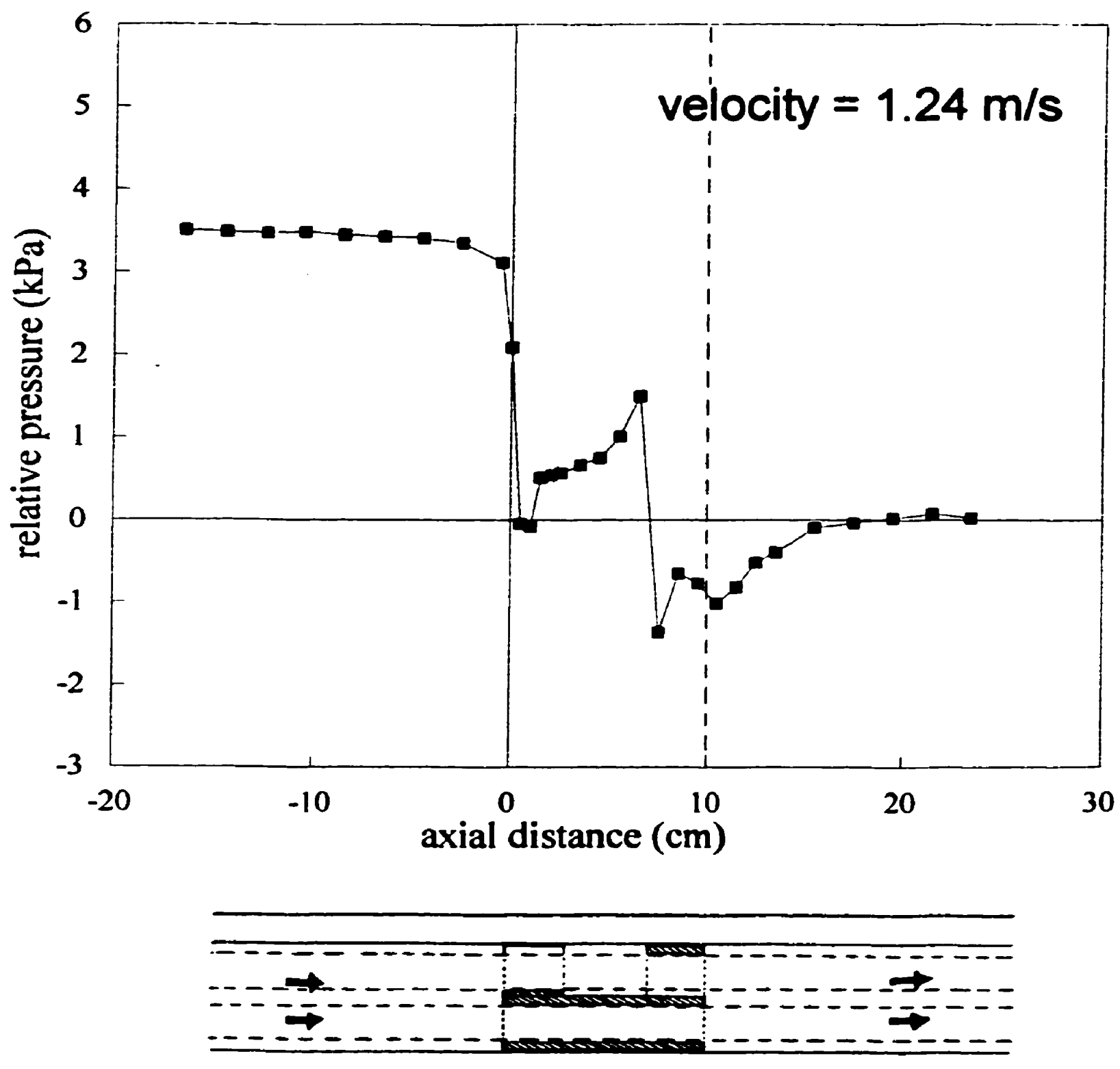
Figure 4.7 (continued)

(c) Pickering-A lattice bars
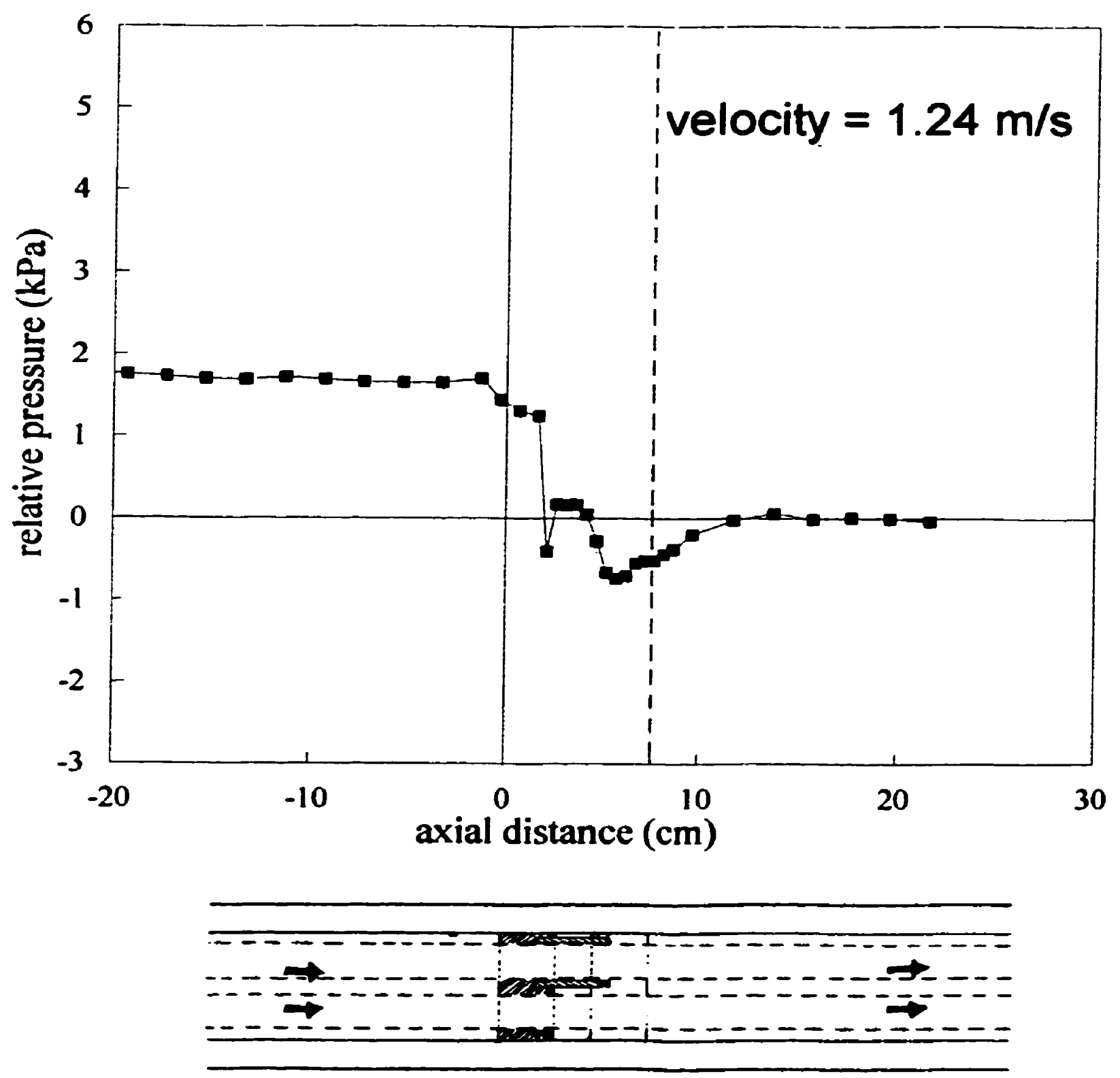
Figure 4.7 (continued)

(d) Wolsong-1 formed bars
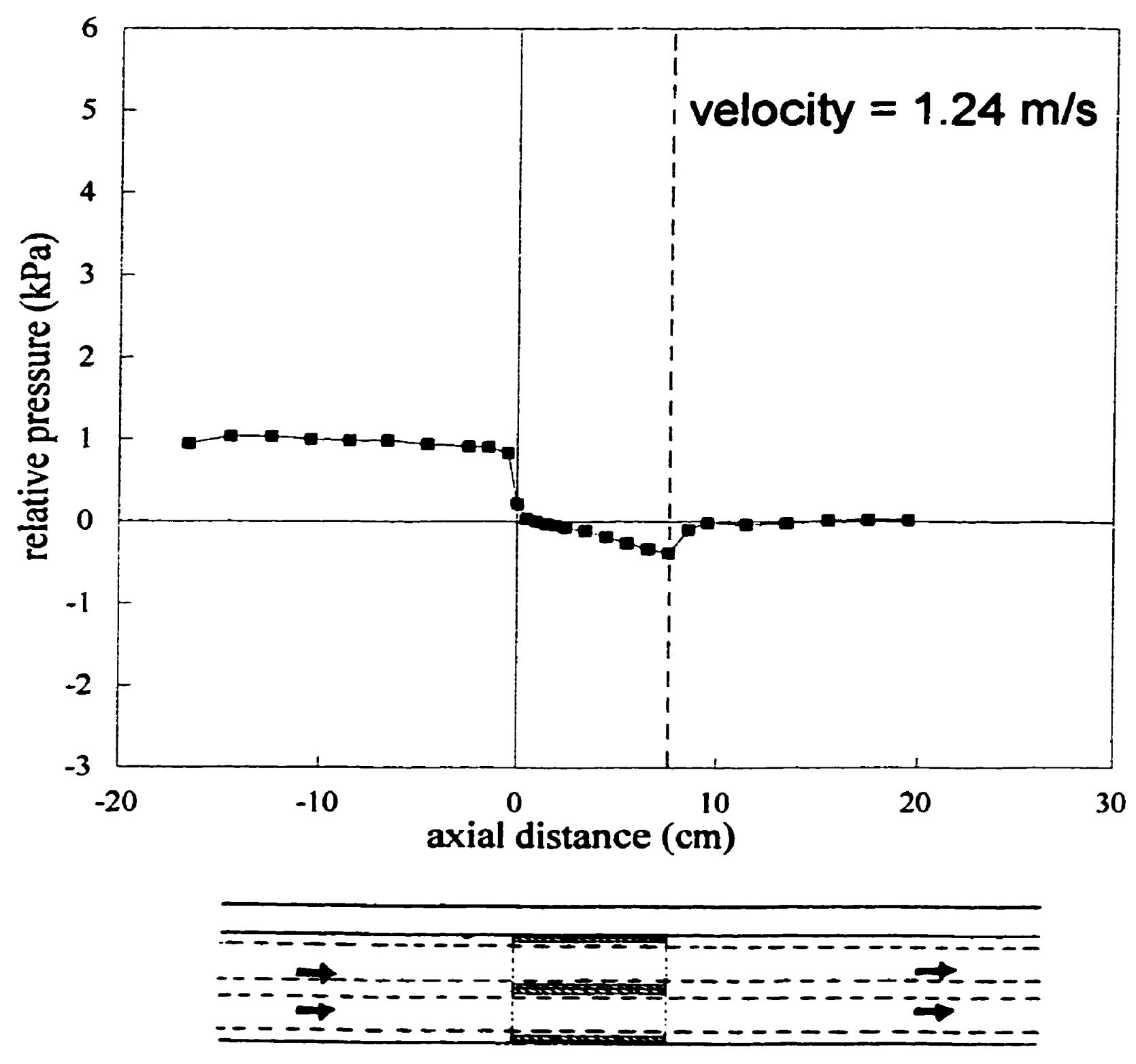
Figure 4.8: Effect of subchannel on Darlington lattice bar pressure profiles
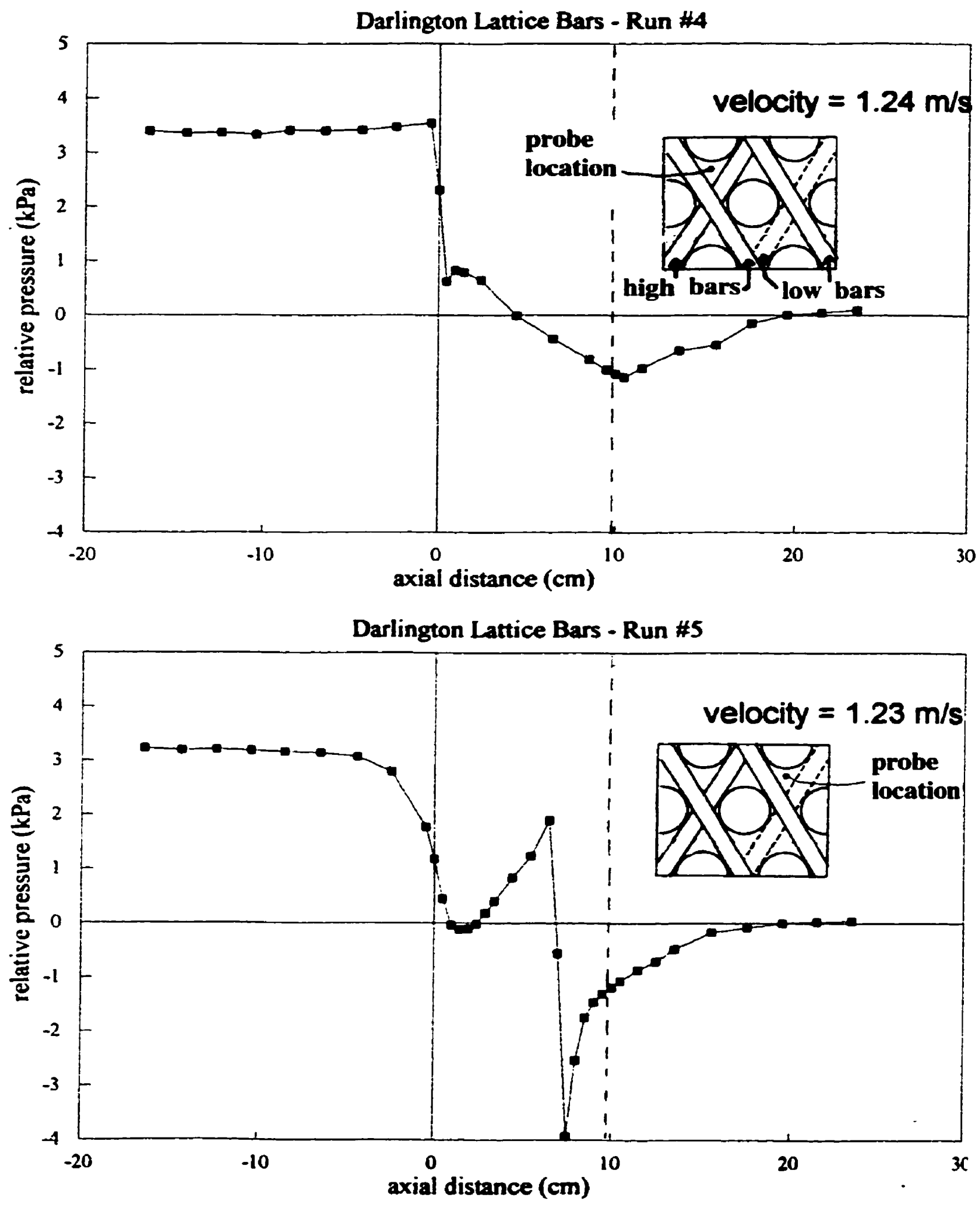
Figure 4.9: Effect of edge sharpness on Darlington lattice bar pressure profiles

Darlingion Latice Dars - edpe eflect

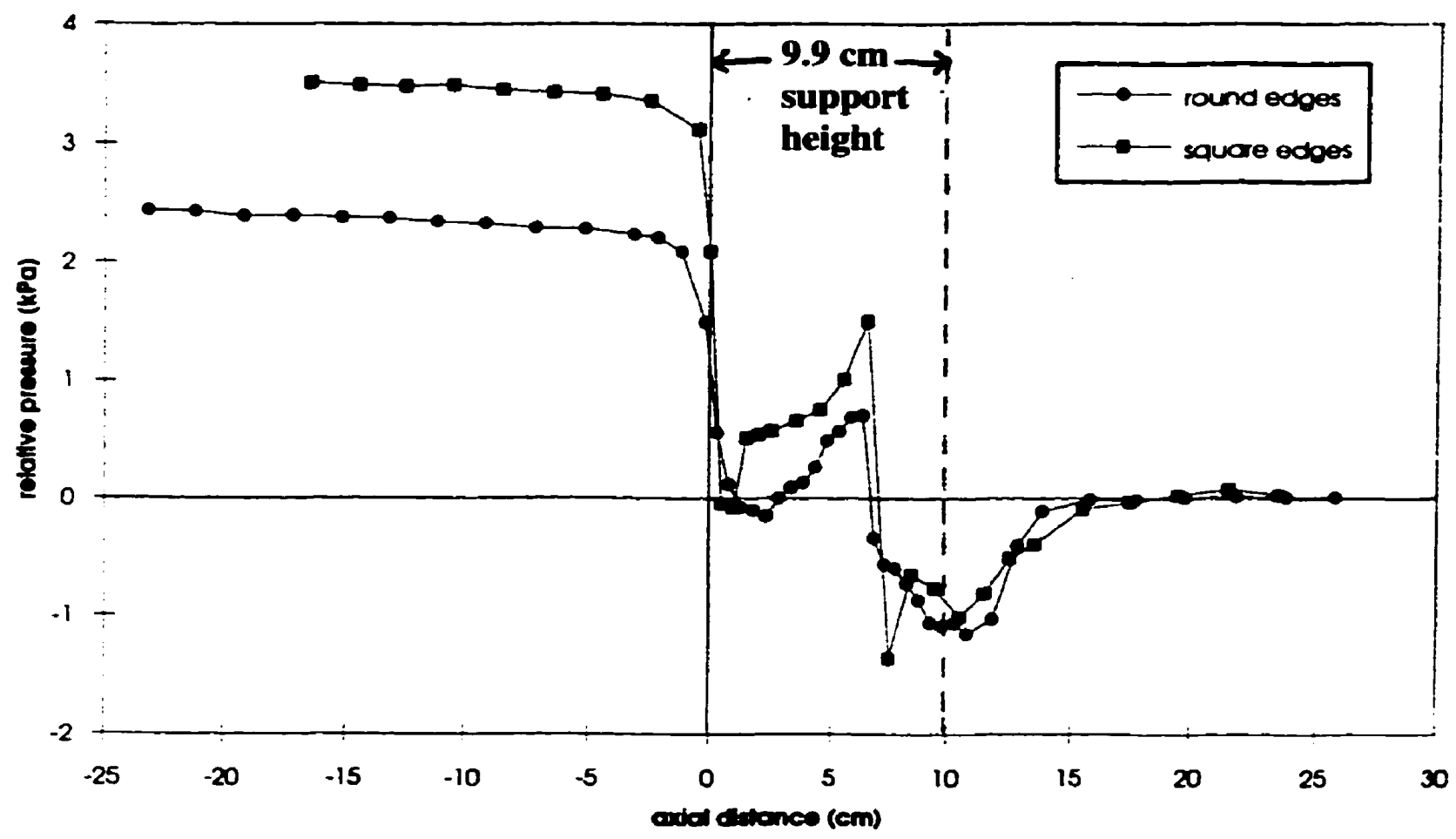


Figure 4.10: LDV apparatus

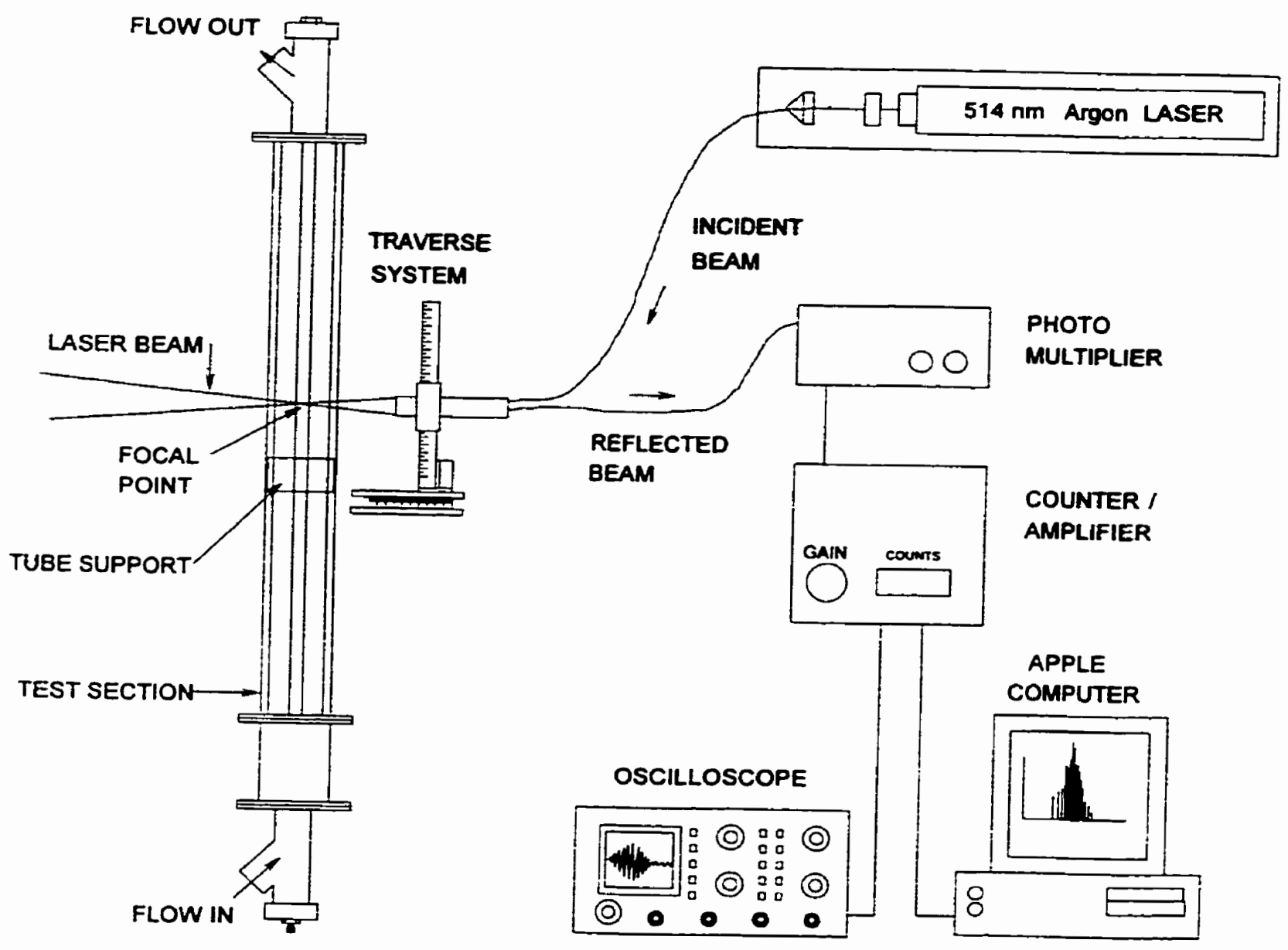


Figure 4.11: Typical output from single velocity measurement

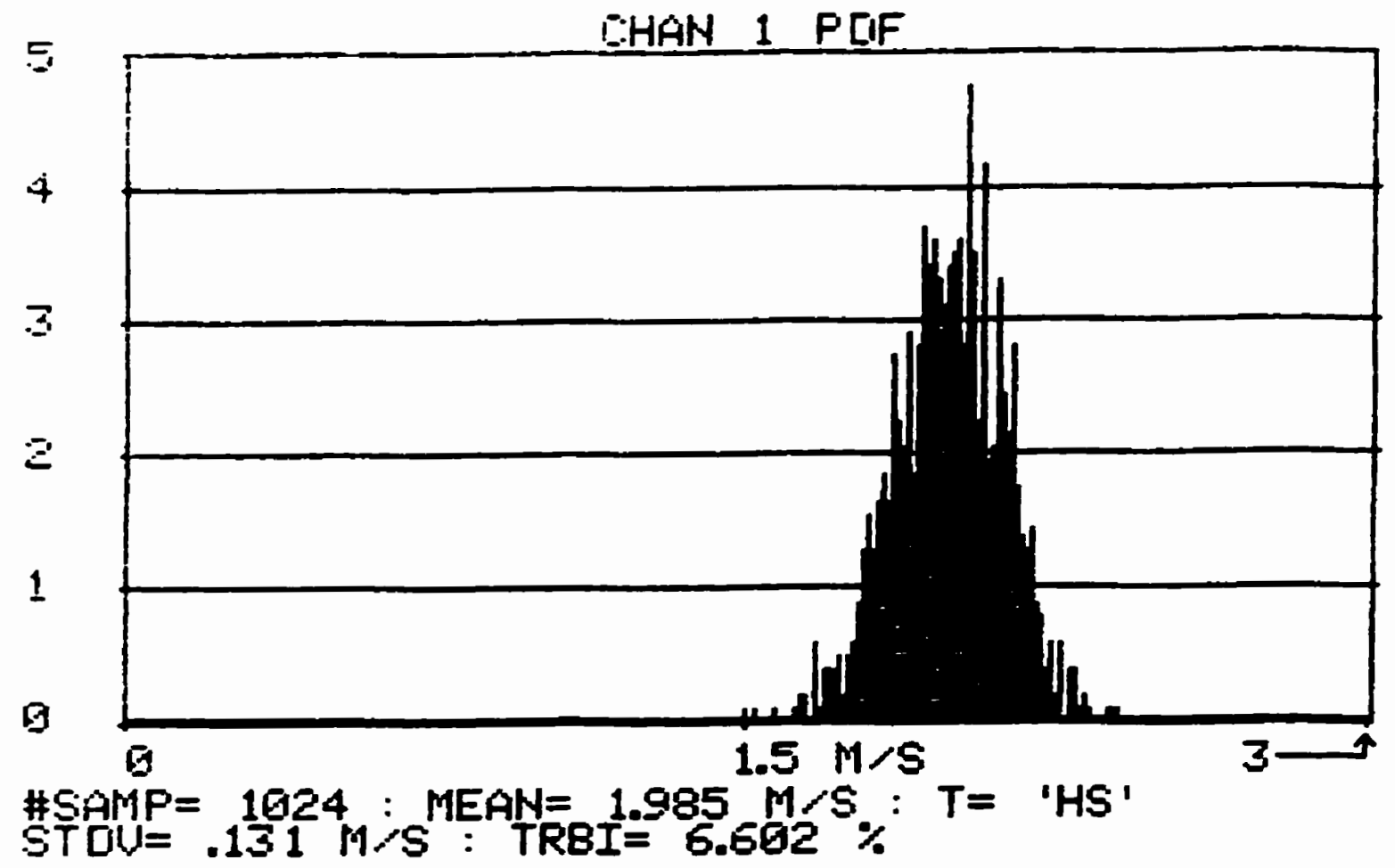


Figure 4.12: Velocity distribution inside sectioned broach hole

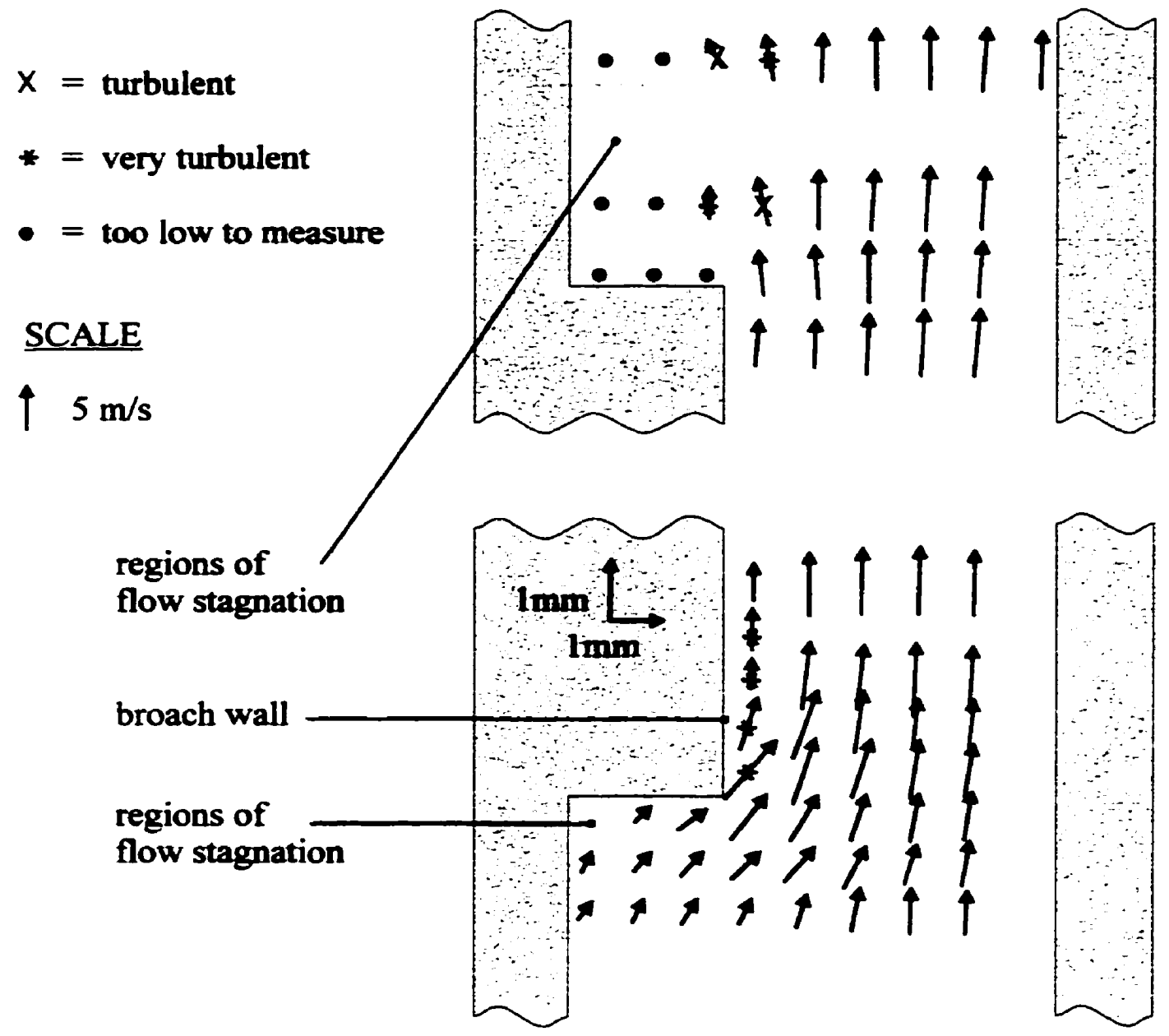


Figure 4.13: Maps of velocity and turbulence intensity

(a) Darlington lattice bars
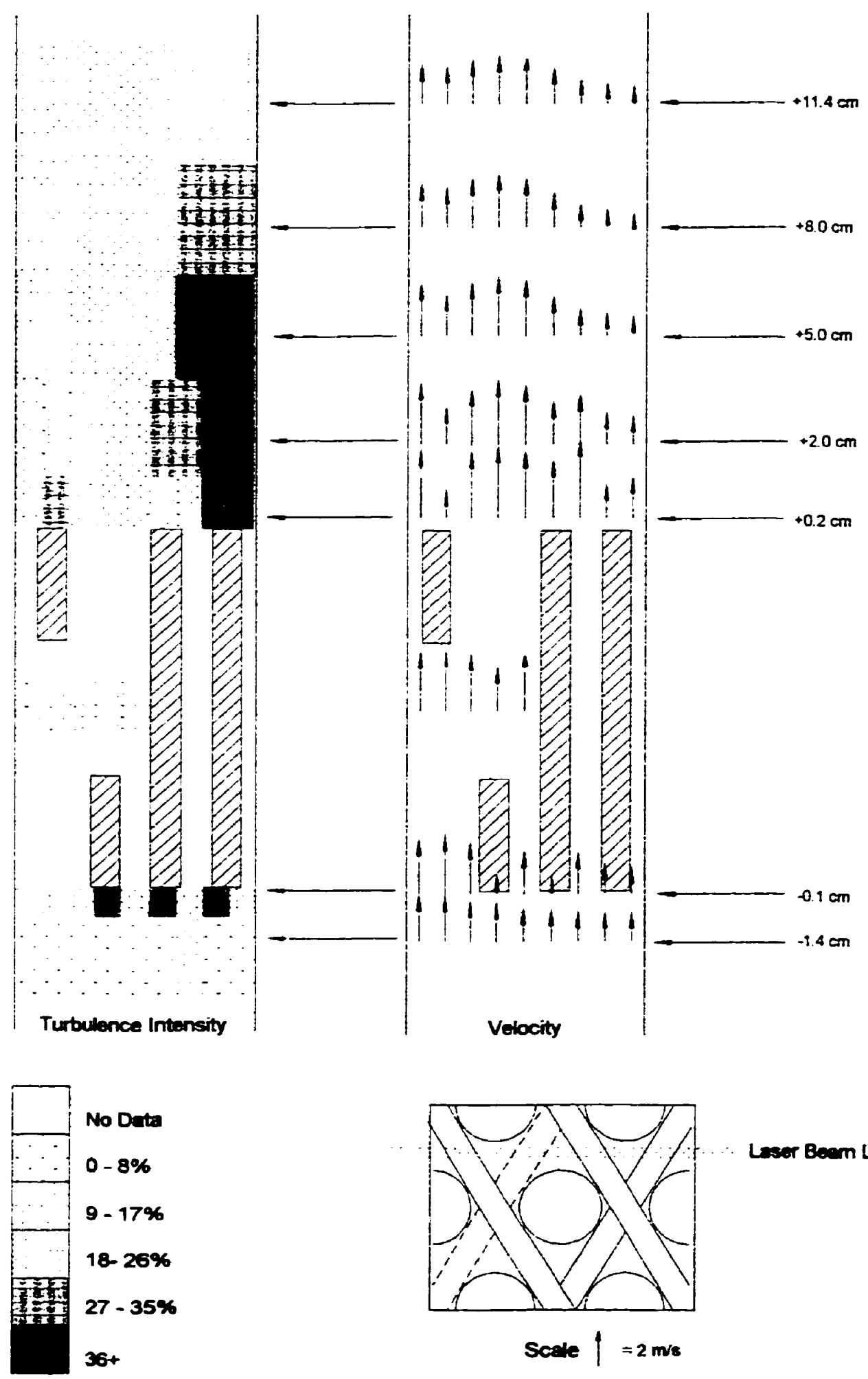

Laser Beam Location 
Figure 4.13 (continued)

(b) Pickering-A lattice bars

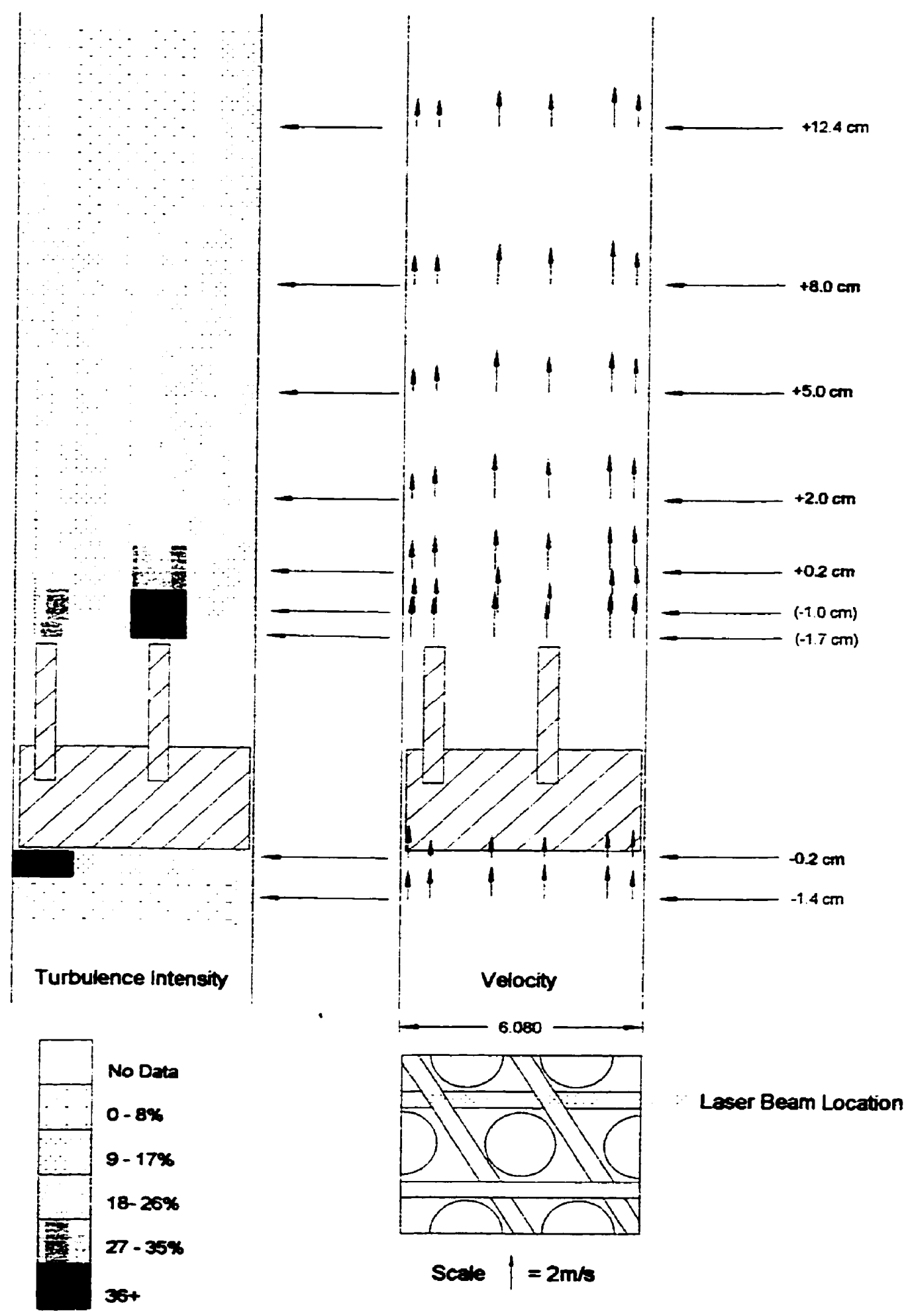


Figure 4.13 (continued)

(c) Wolsong-1 formed bars

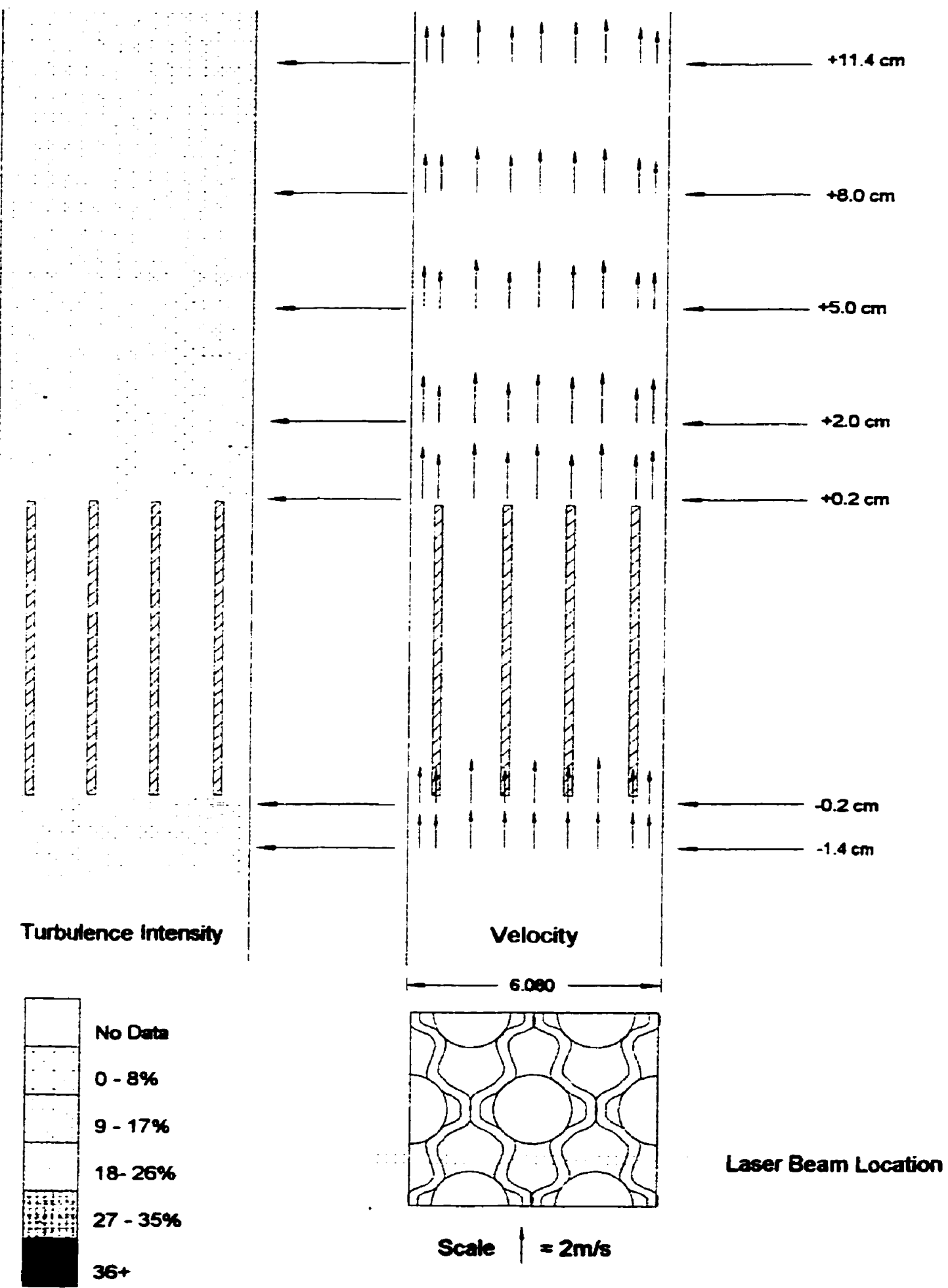


Figure 4.14: Void apparatus

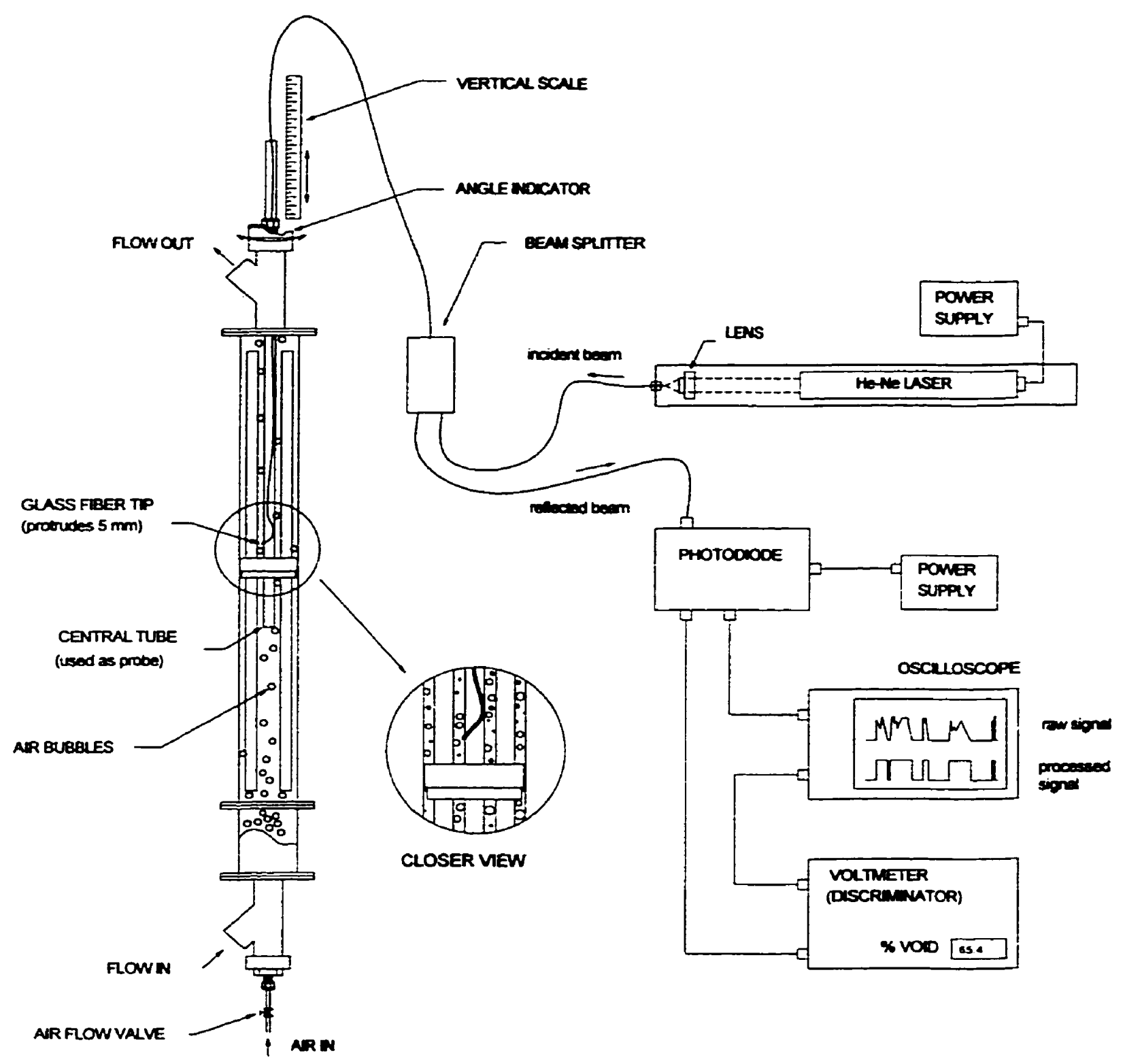


Figure 4.15: Map of void fraction for broached plate

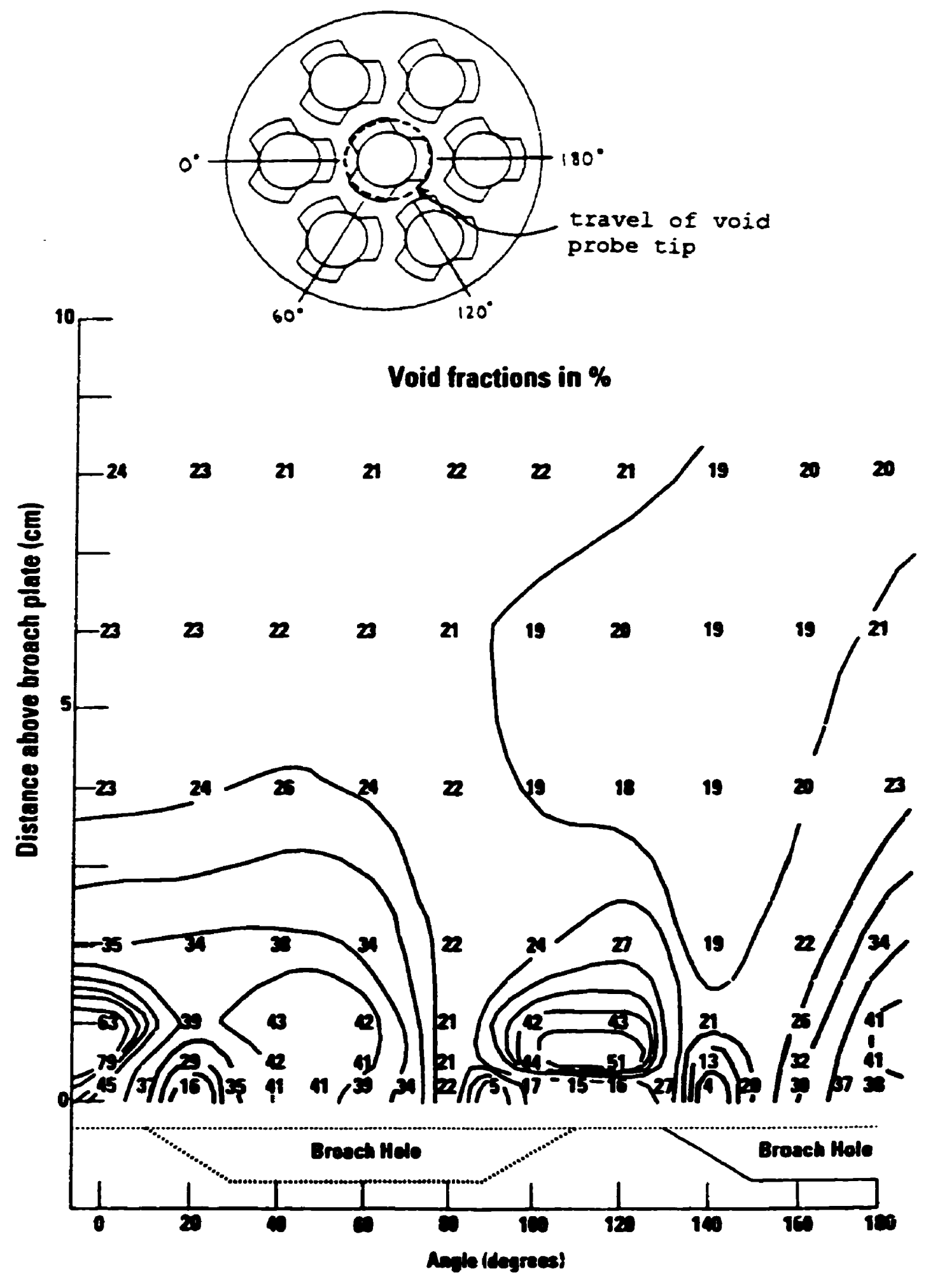




\section{Chapter 5}

\section{EXPERIMENTS IN FREON LOOP}

\subsection{Rationale}

While tests in air/water mixtures were useful in determining the dominant flow patterns, tests in which vapour was generated were necessary to show the thermal and hydraulic behaviour together. Such tests were carried out over a 1-year period (1994-1995) using Freon-11. In simulating the high-pressure, high-temperature SG water, Freon-11 had more representative physical properties (and was therefore a better modeling fluid) than the air/water mixtures of previous tests, as shown in Table 3.1. However, owing to the low pressure of the Freon representation, a greater amount of void was expected for the same quality than in an actual SG (and vice versa).

\subsection{Description of Test Loop}

The tests carried out in the Freon-11 loop used mock-ups of a single SG tube and its associated support. Four types of CANDU SG supports were tested in the Freon loop (see Table 5.1 and Figure 5.1): the Bruce broached plate, CANDU-6 broached plate, Darlington lattice bars, and Wolsong-1 formed bars.

As in the air/water tests (see Section 4.2), the Darlington high-bar intersection region was represented in the mock-up as the "worst case" from the standpoint of flow restriction. Dimensions were scaled up by 20 to $50 \%$ for better observation and to accommodate instruments. The scaling factor for each mock-up was determined by comparing the testsection tube pitch with the appropriate SG tube pitch. As indicated in Table 5.1, the flow-area ratios and height-to-diameter ratios were representative of the SG. 
The test loop is shown in Figures 5.2 and 5.3. The measurements were carried out in Freon- 11 at low temperature $\left(<45^{\circ} \mathrm{C}\right)$ and near-atmospheric pressure. Freon- $11\left(\mathrm{CCIF}_{3}\right)$ was chosen over other refrigerants because its low boiling temperature $\left(23^{\circ} \mathrm{C}\right.$ at atmospheric pressure) allowed thermal behaviour such as flashing* and boiling to be studied without resorting to a high-pressure loop. However, due to stringent environmental regulations that came into effect in 1994 for ozone-depleting substances, releases to atmosphere were prohibited and much effort was needed to make the loop leak-tight. Freon-11 is currently banned from use at CRL.

The Freon-1 1 flow loop delivered a maximum liquid velocity of $\sim 1 \mathrm{~m} / \mathrm{s}$, which is representative of the lower region of the SG. The combination of test fluid properties and scaled-up geometries gave liquid Reynolds numbers that were low only by a factor of about 1.5 compared with the actual SG. Thus, Re values in the Freon tests were more representative of those in the SG than the $\operatorname{Re}$ values in the air/water tests were.

Most of the Freon tests were carried out in "adiabatic" mode, with a two-phase mixture created at the test-section inlet and no further heating beyond that. In this mode, flashing - which is vapour generation due to reduction in pressure and not due to heating-could be observed on its own. Other tests were carried out in "diabatic" mode, with vapour bubbles generated on the heated central tube. In this mode, both flashing and boiling could be observed.

\subsection{Flow Visualization}

The objective of the flow visualization tests was to observe how each support affects the flow along the SG tubes. 


\subsubsection{Results with Adiabatic Flow}

Figures $5.4 \mathrm{a}$ to $5.4 \mathrm{~g}$ show the characteristic liquid/vapour pattern in adiabatic bubbly flow for each support. Video recordings were also produced. The two-phase flow patterns were similar to the patterns seen with air/water bubbly mixtures (see Section 4.3.1). This suggests that the air bubbles had been adequate tracers, and that two-phase adiabatic flow patterns could be represented reasonably well by two-component air/water mixtures.

With all support designs, recirculation eddies appeared at relatively low void fractions $(-15 \%)$ above either the plate (for broached plates) or the bar intersection region (for lattice bars or formed bars). No recirculation occurred above the thin lattice or formed bars themselves; instead, a vapour pocket was present. When heat to the fluid was increased upstream of the test-section to give higher void fractions $(-30 \%)$, the eddies turned into quasi-stable vapour pockets or "tongues". This phenomenon had not been observed with the air/water mixtures.

The broached plates showed very distinct "jetting" in their two-phase patterns: the eddies and vapour pockets were contained between strong mostly-liquid jets coming out of the broach holes. This jetting pattern was very consistent. In contrast, the lattice-bar patterns indicated much mixing, owing to flow redirection by the low bars; results from voidfraction measurements (see Section 5.5) supported this observation. All supports except the Darlington lattice bars ${ }^{4}$ showed distinct flashing at the inlet of the support.

In general, void fractions were much higher downstream of the supports than upstream, owing to a combination of flashing as a result of the relatively large pressure loss across the support and the trapping of void in low-pressure regions. These phenomena would also occur in the actual SG, but to a lesser extent, because less void is produced for the

\footnotetext{
4 Unlike in the air/water tests, the bars of the lattice mock-up for the Freon tests were fully rounded according to design blueprints; the two broached-plate mock-ups and formed-bar mockup were left with sharp $\left(90^{\circ}\right)$ edges.
} 
same quality change under the higher-pressure conditions. For example, assuming the phases are homogeneously mixed, an increase in quality from 0.5 to $1 \%$ in the Freon test loop would increase the void fraction from 40 to $57 \%$ (a difference of $17 \%$ in absolute terms); in the SG, the corresponding increase in void fraction would be from 15 to $26 \%$ (an $11 \%$ difference).

\subsubsection{Results with Diabatic Flow}

After the adiabatic tests, the Freon loop was modified to allow vapour generation on the central tube. This tube was heated by flowing hot service water along the inside.

In general, the diabatic flow patterns featured greater turbulence. For the lattice bars, this meant increased mixing beyond the already strong cross-flow: whether bubbles were generated by flashing or by boiling, sufficient mixing took place at the support that the bubble origin did not influence the dominant patterns. Diabatic patterns with the broached plate (and, to a much lesser extent, the formed bars) were less stable than the corresponding adiabatic ones. Liquid jets and vapour tongues were less distinct, presumably because of distuption from bubbles generated on the tube in these regions.

When the broached plates were inadvertently subjected to slug flow, the jet-and-tongue pattern was found to quickly re-establish itself between the flow perturbations. This support design appeared to have a strong flow-stabilizing effect, regardless of the flow regime or the mode of heating.

\subsection{Pressure Loss and Axial Pressure Profile}

The objective of the pressure-loss tests was to obtain axial pressure profiles in singlephase and two-phase flow. Details of the measured profiles would give clues regarding 
potential fouling sites. The experimental profiles would also be useful for comparing with calculated profiles, to help validate the pressure loss model used in analytical work (see Section 7.3.5).

\subsubsection{Experimental Method}

The pressure-loss tests were conducted under adiabatic conditions. As shown in Figure 5.5, axial pressure profiles were obtained using a sliding pressure probe connected to a differential pressure transducer, or DP cell. This apparatus was similar to that of the air/water test (see Section 4.4.1), except that the probe was housed inside the central tube, which represented an SG tube. By sliding the central tube up or down, an axial profile of the pressure as a function of elevation could be obtained.

The pressure-loss measurements were complicated by the fact that Freon-11 vaporizes at $23^{\circ} \mathrm{C}$ at atmospheric pressure. Many efforts were made to avoid vapour pockets in the impulse lines to the DP cell, especially during summer testing. For example, the pressure probe inside the central tube was shrouded by a larger diameter tube carrying cooling water (see Figure 5.5). Also, the impulse lines were bled into a chilled storage tank or backflushed to eliminate trapped vapour.

A thermocouple installed between the cooling jacket and the end of the probe (which was open to the flow of Freon) was used to gauge whether the Freon was sufficiently subcooled within the impulse line. Drifting or non-repeatable pressure data were other indications that vapour was trapped in the lines, and such data were discarded.

Once the pressure readings appeared to be stable, a reading at each elevation was obtained by collecting and time-averaging multiple signals using data acquisition software. Time-averaging was important because of the high DP cell sensitivity used, and because of random fluctuations (turbulence) in the flow. 


\subsubsection{Results for Single- and Two-Phase Flow}

Figures 5.6a to 5.6c show typical single-phase and two-phase pressure profiles for the supports. [Measurements were not made with the Wolsong-1 formed bars, as the pressure probe was damaged prior to completion.] The two-phase profiles were obtained at liquid velocities between 0.80 and $1.02 \mathrm{~m} / \mathrm{s}$ (for the free-span region, and calculated from the mass flow rate measured in single-phase). The main contributors to the twophase profiles are:

1. friction, obstruction, and area changes,

2. acceleration due to increasing void fraction (due to flashing of the saturated liquid) as a result of pressure drop and elevation change, and

3. difference in static head due to density difference between the (warm) two-phase flowing fluid and the (cool) single-phase stagnant fluid in the impulse lines. ${ }^{5}$

For some runs, void fractions were measured simultaneously with the relative pressures, to establish the approximate quality conditions and to provide void profiles for two-phase pressure drop calculations. Void profiles are discussed in Section 5.5.

For all three supports, the pressure recoveries within and beyond the support are more gradual in two-phase than in single-phase at the same liquid inlet velocities. The region of minimum pressure that corresponds to the vena contracta region is therefore more extensive. This has implications for fouling, because of potential precipitation of cementing agents in low-pressure zones (as discussed in more detail in Chapter 6).

Also noteworthy is the larger pressure loss at the support inlet under two-phase conditions compared with single-phase, and the larger pressure gain at the support outlet. This

\footnotetext{
${ }^{5}$ When differential pressures are measured, the static head in the test-section is cancelled out by the static head in the impulse line, except when the fluids are at different temperatures and hence densities.
} 
illustrates the requirement for "two-phase multipliers" in the pressure loss model (described in Section 7.3.5). The lower overall loss in two-phase (compared to singlephase) seems counter-intuitive; however, this is a result of pressure gain from static head differences between the impulse lines and the test-section (which carried the lower density two-phase fluid).

\subsubsection{Comparison with Calculated Pressure Profile}

Figure 5.7 shows a comparison between experimental and calculated pressure loss profiles. A two-phase profile obtained for the CANDU-6 broached plate was chosen for the comparison, as it had the most complete information, including an axial void profile (see Section 5.5), and the simplest geometry. Section 7.3.5 outlines the method used for the calculation. Figure 5.8 shows separately the components that contribute to the calculated pressure profile: friction and obstruction, acceleration due to void generation, and the static head difference described in the previous section.

It is clear from Figure 5.7 that the model underestimates the two-phase losses, as it did in the single-phase profiles collected with the air/water loop (see Section 4.4.3). The overall pressure loss between upstream and downstream plateaus is low by $32 \%$, and the maximum loss between upstream plateau and point of minimum pressure is low by $16 \%$. Also, the profile within the support is not captured exactly. Possible reasons for these differences are:

- In this application, the model did not take into account developing lengths. Typical developing lengths for single-phase turbulent flow suggest that flow is developing over the entire height of the support. Under these conditions, friction factors (for example) are higher. Also, the developing length is typically longer in two-phase flow than in single-phase. 
- In the model, the vena contracta region is assumed to cease half-way into the broach hole, and the flow expansion at the outlet is assumed to be immediate. In reality, the boundary-layer re-attachments and the associated pressure changes are gradual.

- The shape of the pressure-loss profile is heavily dependent on the measured void profile, which is a crude representation of a very non-uniform void distribution.

- The two-phase flow was assumed to be homogeneous. This may be invalid within the support and immediately beyond, where void measurements revealed large pockets of vapour. However, there is currently no obvious alternative for this assumption.

\subsection{Void Fraction}

The objective of the void-fraction measurements was to determine the liquid/vapour distribution in the vicinity of the supports. This, in turn, would indicate where particulates could deposit on the support, or where solubles could precipitate as a result of flashing of saturated liquid.

\subsubsection{Experimental Method}

The void-fraction measurements were conducted under adiabatic conditions. They used the same laser and probe techniques as in the air/water tests (see Section 4.6.1), i.e., with optical fibers contained within the central tube, with only their tips immersed in the flow. As with the pressure-loss measurements, these techniques were adapted or developed during the air/water tests and were found to work well.

As shown in Figure 5.9, one fiber tip (or probe) was located $2 \mathrm{~mm}$ away from the tube surface, the other at $5 \mathrm{~mm}$ ( $6 \mathrm{~mm}$ for the Bruce broached plate). The central tube could 
be twisted and moved vertically, such that a two-dimensional map (angle and elevation) was obtained for both the $2 \mathrm{~mm}$ and $5 \mathrm{~mm}$ probe locations. The $2 \mathrm{~mm}$ probe could move within the openings in the support and therefore access the regions below the support.

\subsubsection{Results for Local Void Fraction}

Figures 5.10 to 5.13 are examples of the measured void fraction-values on the maps indicate \% void - as a function of axial elevation ( $y$-axis) and rotation angle ( $x$-axis). As these void maps were created using contouring computer software, some fine details may be artifacts of the interpolation method used. ${ }^{6}$ However, the contouring does represent the overall void distribution well.

The measurements were taken at a single-phase inlet velocity of $\sim 1.0 \mathrm{~m} / \mathrm{s}$ (as with the pressure-loss tests). As they were obtained under ambient pressure conditions, the corresponding qualities were very low. For example, at the highest average void fraction achieved in the test-section $(-40 \%)$, the mass quality is calculated to be only $\sim 0.5 \%$ in the test-section, compared to $\sim 2 \%$ quality under a SG pressure of 4.5 MPa. However, from the standpoint of flow patterns, it is the void fraction that is more relevant. $40 \%$ void is representative of the lower regions of the SG.

The first map, Figure 5.10, was obtained for the Bruce broached plate, and indicates pockets of higher void directly above the web of the plate, and lower-void flow passing through the broach hole. [A bird's-eye diagram is included in the figure to show the path of the optical probe.] This is shown more clearly in maps for the CANDU-6 broached plate (Figures $5.11 \mathrm{a}$ and $5.11 \mathrm{~d}$ ). The void measurements reveal a symmetric void pattern above the plate where eddies or vapour pockets were observed visually. The presence of

\footnotetext{
${ }^{6}$ Because measurements were taken at discrete locations in the flow field, and the points were not always spaced at equal intervals, the appearance of the graphs were influenced by the method of interpolating between the points.
} 
void in this region can be explained by the tendency of the lighter (less dense) vapour to preferentially seek low-pressure regions, as less force is required-compared with liquid - to accelerate it or change its direction. In other words, the flowing liquid has more momentum and keeps to a mainstream jet while the vapour gets drawn into the lowpressure wake region.

The void pocket is clearly confined to the region directly above the plate. Visually, it appeared to be separated from the plate by a thin $(0.5$ to $1 \mathrm{~mm})$ liquid film. Comparison between Figures $5.11 \mathrm{a}$ and $5.11 \mathrm{c}$ reveal that the void pocket grows in height with increasing void. More importantly, recirculation eddies observed under medium void conditions (Figure 5.11a) grew into wavering vapour tongues under high void (Figure $5.11 \mathrm{c})$. It was from void measurements that these "tongues", seen earlier during flow observation (Section 5.3.1), could be identified as vapour and not liquid (-both are transparent). Flashing was observed visually at the inlet of both Bruce and CANDU-6 broached plates, but the thin void pocket on the broach hole wall could not be accessed by the void probe.

Void maps for the Darlington lattice bars (Figures 5.12a to 5.12d) are less symmetric, mainly because the asymmetry of the design itself promotes more cross-flow and flow mixing than with the symmetric broached plates. Again, high void was detected immediately downstream of all the bars. However, the pockets were less stable than those of the broached plates, owing to greater flow mixing. Small regions of higher void were also found beside the bars; rather than regions of flashing, these seem to be stagnant zones (which preferentially attract vapour) that are created by cross-flow and flow separation.

Void maps for the Wolsong-1 formed bars (Figures 5.13a to 5.13d) again featured high void above the bars. Flow recirculation above the bars was minimal. Distinct flashing was detected at the support inlet (Figure 5.13a). Such flashing is indicative of a sudden 
pressure drop at the support inlet although, as stated in Section 5.3.1, the change in void fraction would be less under the high-pressure conditions of the SG.

It appears from both void maps and visual observation that the smaller the surface area normal to the flow (and hence the smaller the flow area ratio), the less room there is for downstream recirculation and the more likely bubbles will form a fixed vapour pocket.

\subsubsection{Results for Axial Void Profile}

An axial void profile, Figure 5.14, was constructed from the CANDU-6 void maps in Figures $5.11 \mathrm{c}$ and $5.11 \mathrm{~d}$, and from local void fraction measurements taken during pressure loss runs under similar loop conditions $(-1.0 \mathrm{~m} / \mathrm{s}$ liquid velocity, $\sim 40 \%$ maximum void). The overall increase in void fraction with elevation is the result of flashing of the liquid as the pressure is reduced, under these adiabatic conditions. As explained in Section 5.3.1, the flashing is more pronounced in the Freon loop than in the SG, but it serves to simulate the increase of void due to boiling in the SG.

At high elevations ( $>15 \mathrm{~cm}$ from support inlet), the single local void measurements were plotted. These were adequate because the radial void distribution well past the support was nearly uniform. Closer to the support, the distribution was typically very nonuniform; however, values obtained by averaging measurements taken at different radial positions on the void maps link well with the local measurements taken higher up. Furthermore, the void profile properly represents the high concentration of void immediately beyond the support ( $>3 \mathrm{~cm}$ in Figure 5.14) caused by trapping of vapour in recirculation and low-velocity regions. 


\subsection{Observation under SG Conditions}

An experiment was carried out in a reduced-scale steam generator which operated under high-pressure (3.5 MPa), high-temperature $\left(242^{\circ} \mathrm{C}\right)$ conditions. The test-section consisted of 6 tubes and 3 broached-plate supports, with small (4-cm diameter) viewing windows in line with the highest support. The objective was to observe the flow patterns downstream of the support, for comparison with patterns observed with the laboratory modeling fluids.

Flow qualities in the range of 0 to $20 \%$ were achieved. Viewing was difficult because the windows were small and often obscured by particles from fouling experiments. It was possible, however, to see patterns of recirculating two-phase flow, in which the flow tended to jet out of the broach holes and the bubbles lingered over the support. This is consistent with the dominant flow patterns in all laboratory tests.

The next Chapter describes the postulated correlations between fouling patterns observed in the field and flow patterns observed in the laboratory. 
Table 5.1: Dimensions of mockups for Freon and comparison with actual

\author{
Bruce Broached Plate \\ tube pitch [m] \\ tube diameter [m] \\ broached plate height [m] \\ support flow area per tube ${ }^{1}\left[\mathrm{~m}^{2}\right]$ \\ support wet perimeter per tube [m] \\ support equivalent diameter ${ }^{2}[\mathrm{~m}]$ \\ flow-area ratio ${ }^{3}$ \\ porosity $^{4}$ \\ height to diameter ratio \\ liquid $\operatorname{Re}$ within support $[-]^{5}$

\section{CANDU-6 Broached Plate} \\ tube pitch [m] \\ tube diameter [m] \\ broached plate height [m] \\ support flow area per tube $\left[\mathrm{m}^{2}\right]$ \\ support wet perimeter per tube [m] \\ support equivalent diameter [m] \\ flow-area ratio \\ porosity \\ height to diameter ratio \\ liquid $\operatorname{Re}$ within support [-]
}

\section{Bruce A\&B mock-up scale factor}

$\begin{array}{lll}0.0203 & 0.0278 & 1.37 \\ 0.0130 & 0.0191 & 1.47 \\ 0.0254 & 0.0380 & 1.50 \\ 0.0000966 & 0.000332 & \\ 0.0891 & 0.222 & \\ 0.00434 & 0.00597 & \\ 2.39: 1 & 2.40: 1 & \\ 0.42 & 0.42 & \\ 5.85 & 6.37 & \\ 3.3 \times 10^{4} & 2.4 \times 10^{4} & 0.74\end{array}$

CANDU-6 mock-up scale factor

$\begin{array}{lll}0.0241 & 0.0290 & 1.20 \\ 0.0159 & 0.0191 & 1.20 \\ 0.0254 & 0.0305 & 1.20 \\ 0.000186 & 0.000455 & \\ 0.0110 & 0.230 & \end{array}$

$0.0110 \quad 0.230$

$0.00678 \quad 0.00790$

$1.64: 1 \quad 1.73: 1$

$0.61 \quad 0.58$

$3.75 \quad 3.86$

$5.1 \times 10^{4} \quad 3.2 \times 10^{4} \quad 0.63$

continued

1 measured at mid-point of support

2 equiv diameter $=4 \times$ (flow area per tube) / (wet perimeter per tube)

3 flow-area ratio $=$ (approach region flow area) / (support region flow area)

4 porosity $=1 /($ flow-area ratio $)$

5 determined for $u_{\mathrm{f}}=1.0 \mathrm{~m} / \mathrm{s}$, with fluid properties $\rho_{\mathrm{f}}$ and $\mu_{\mathrm{f}}$ taken from Table 3.1 , columns 2 and 3 , and equiv diameter as in footnote 2

6 assumes lattice consists of only high bars

7 assumes lattice consists of only low bars; dimensions are taken at mid-point of one set of low bars

8 determined at mock-up mid-point where only high bars are present (resembles lowbar-only geometry marked with Footnote 7 
Table 5.1 (continued)

\section{Darlington Lattice Bars}

tube pitch [m]

tube diameter [m]

lattice bar thickness [m]

lattice bar height (high / low) [m]

region of applicability

support flow area per tube $\left[\mathrm{m}^{2}\right]$

support wet perimeter per tube [m]

support equivalent diameter [m]

flow-area ratio

porosity

height to diameter ratio

liquid $\operatorname{Re}$ within support [-]
Wolsong 2-4 \&

Darlington

mock-up scale factor

$\begin{array}{lcl}0.0245 & 0.0290 & 1.18 \\ 0.0159 & 0.0191 & 1.20 \\ 0.00439 & 0.00635 & 1.45 \\ 0.080 / 0.025 & 0.099 / 0.031 & 1.2\end{array}$

high bars $^{6}$ low bars ${ }^{7}$ middle $^{8}$

$\begin{array}{lll}0.000121 & 0.000199 & 0.000616\end{array}$

$\begin{array}{lll}0.134 & 0.0982 & 0.223\end{array}$

$\begin{array}{lll}0.00362 & 0.00811 & 0.0111\end{array}$

$2.52: 1 \quad 1.53: 1 \quad 1.52: 1$

$\begin{array}{lll}0.40 & 0.65 & 0.66\end{array}$

$\begin{array}{lll}22.1 & 3.08 & 8.92\end{array}$

$6.1 \times 10^{4} \quad 4.5 \times 10^{4} \quad 0.74$

Wolsong-1

mock-up scale factor

tube pitch [m]

0.0238

0.0302

1.27

0.0159

0.0191

1.20

0.00157

0.0024

1.5

0.0508

0.076

1.5

0.000207

0.000618

0.118

0.317

0.00702

0.00780

1.41:1

$1.45: 1$

0.71

0.69

7.24

9.74

$5.3 \times 10^{4}$

$3.2 \times 10^{4}$

0.60 
Figure 5.1: Mockups tested in Freon

SCALE : CANDU-6 broached plate mockup is $3 \mathrm{~cm}$ tall.

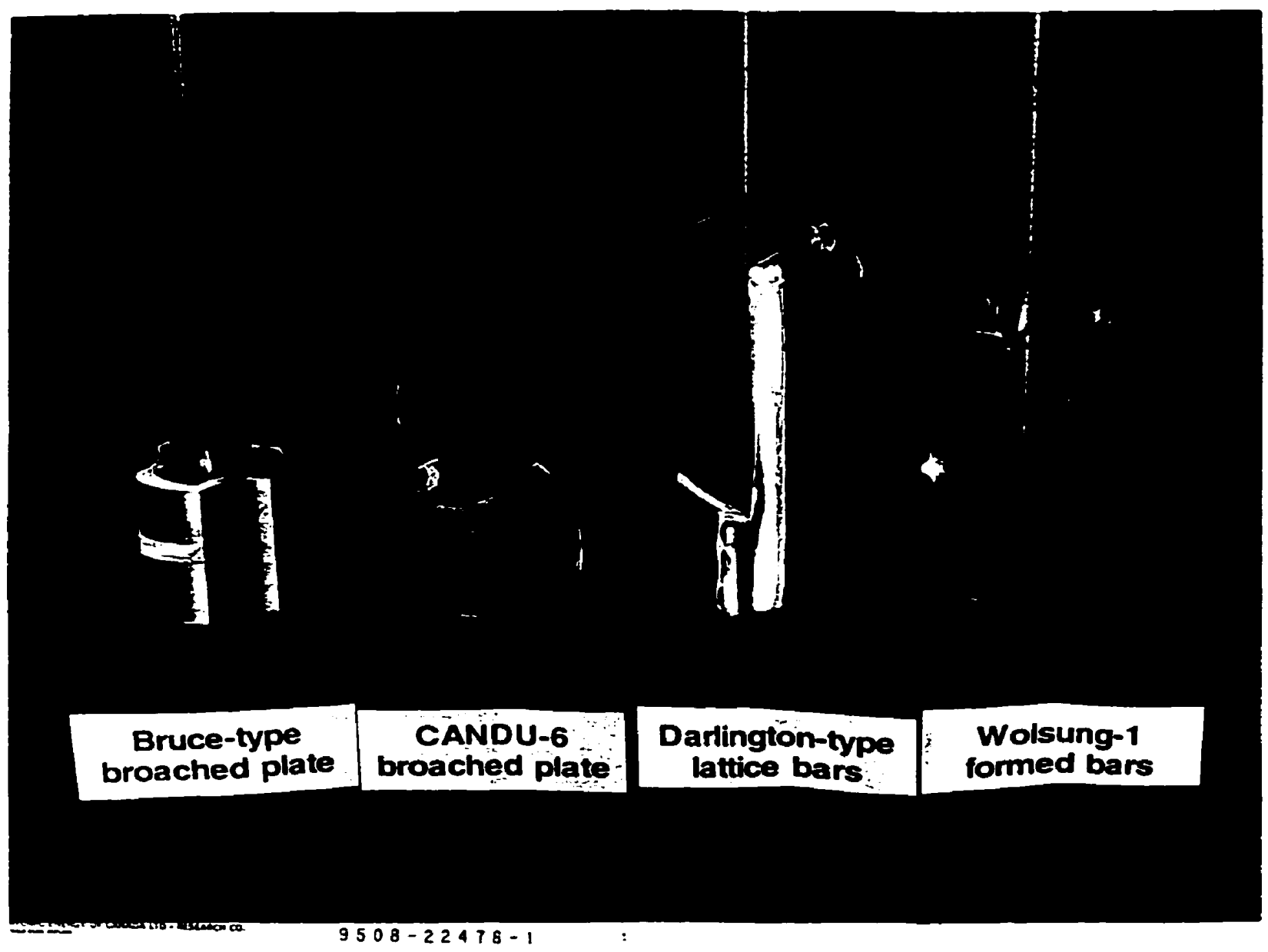


Figure 5.2: Freon test loop

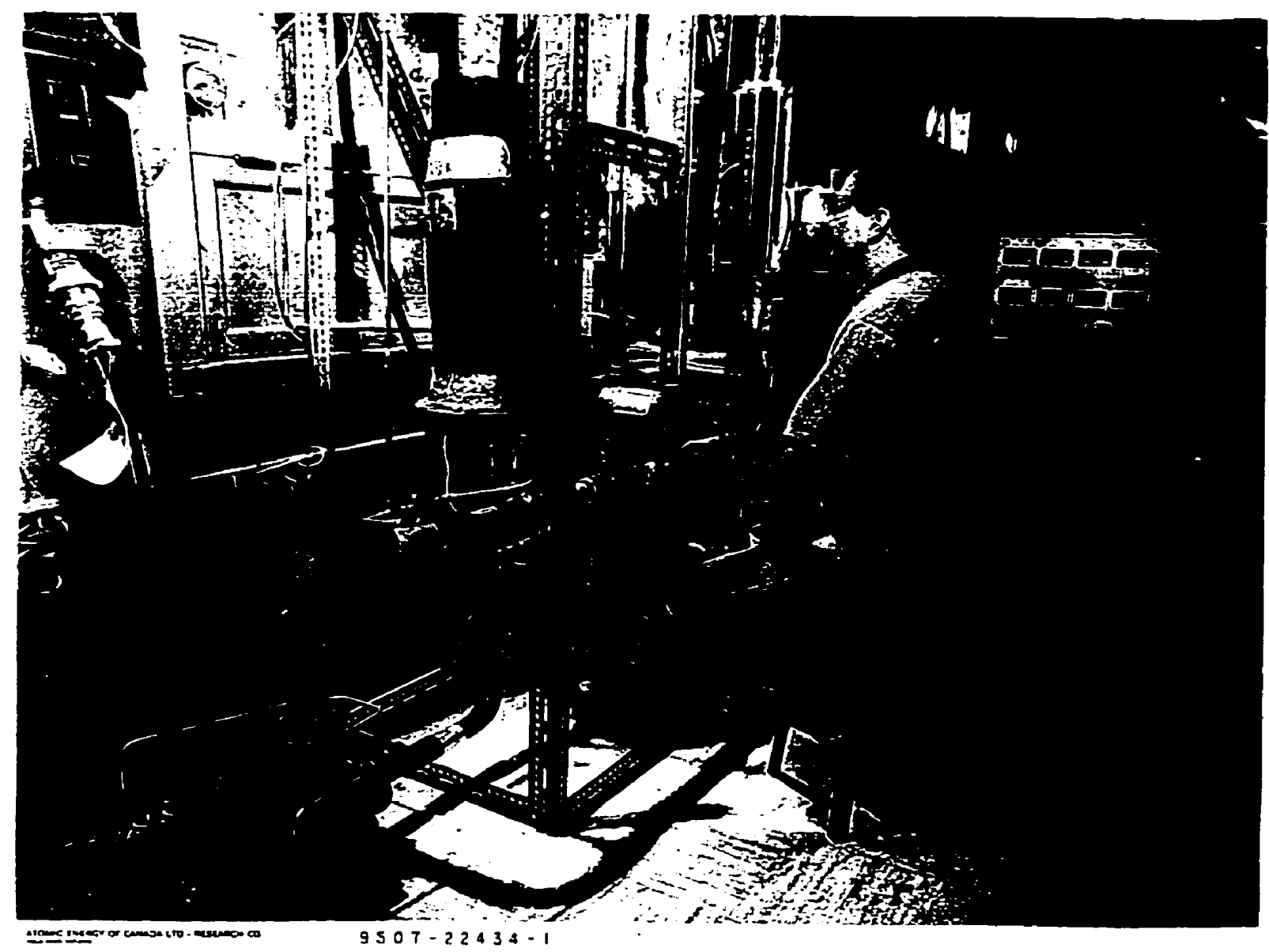


Figure 5.3: Schematic of Freon-11 test loop

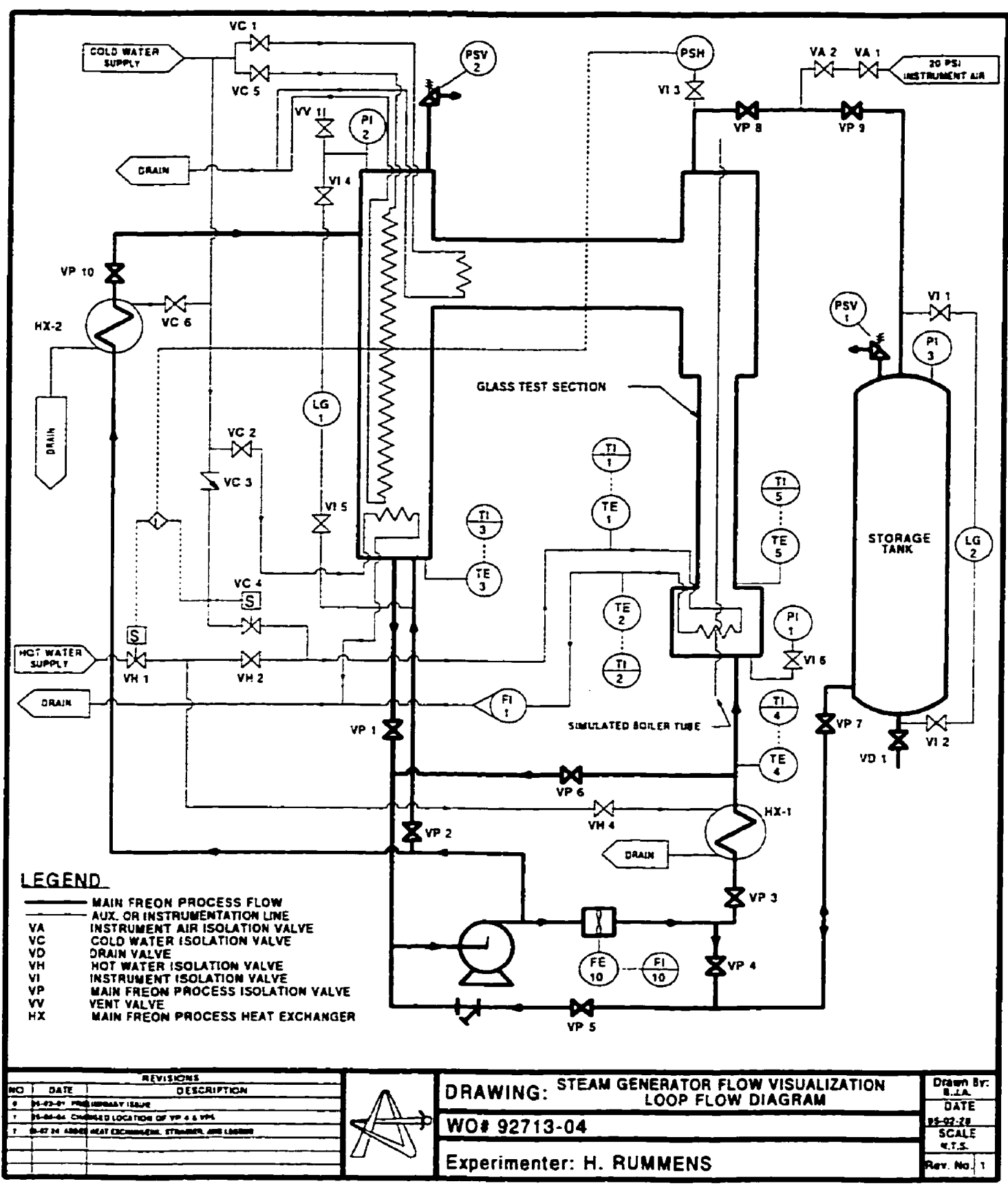


Figure 5.4: Observed flow patterns - clean supports

(a) Bruce broached plate

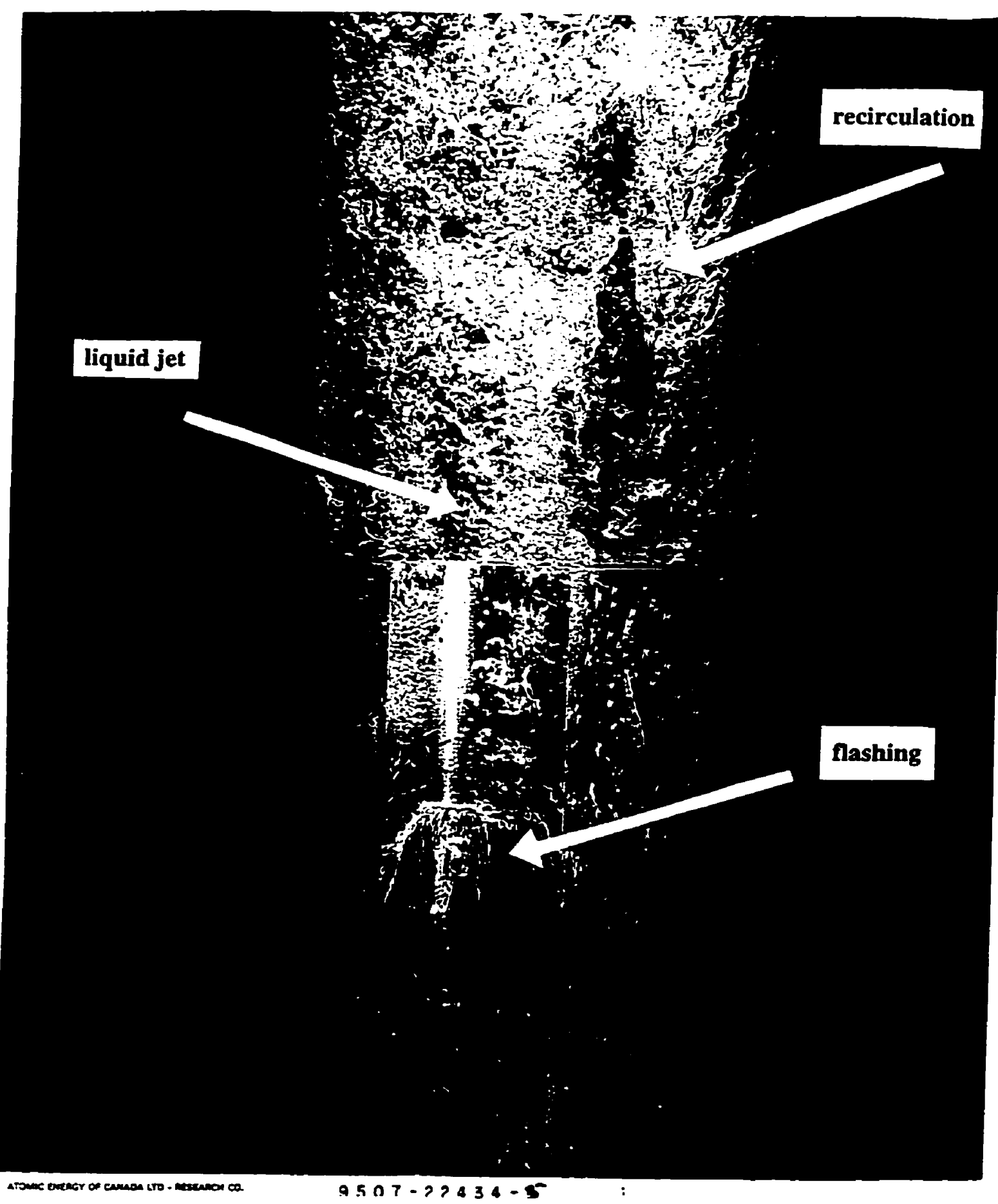


Figure 5.4 (continued)

(b) CANDU-6 broached plate, low void fraction

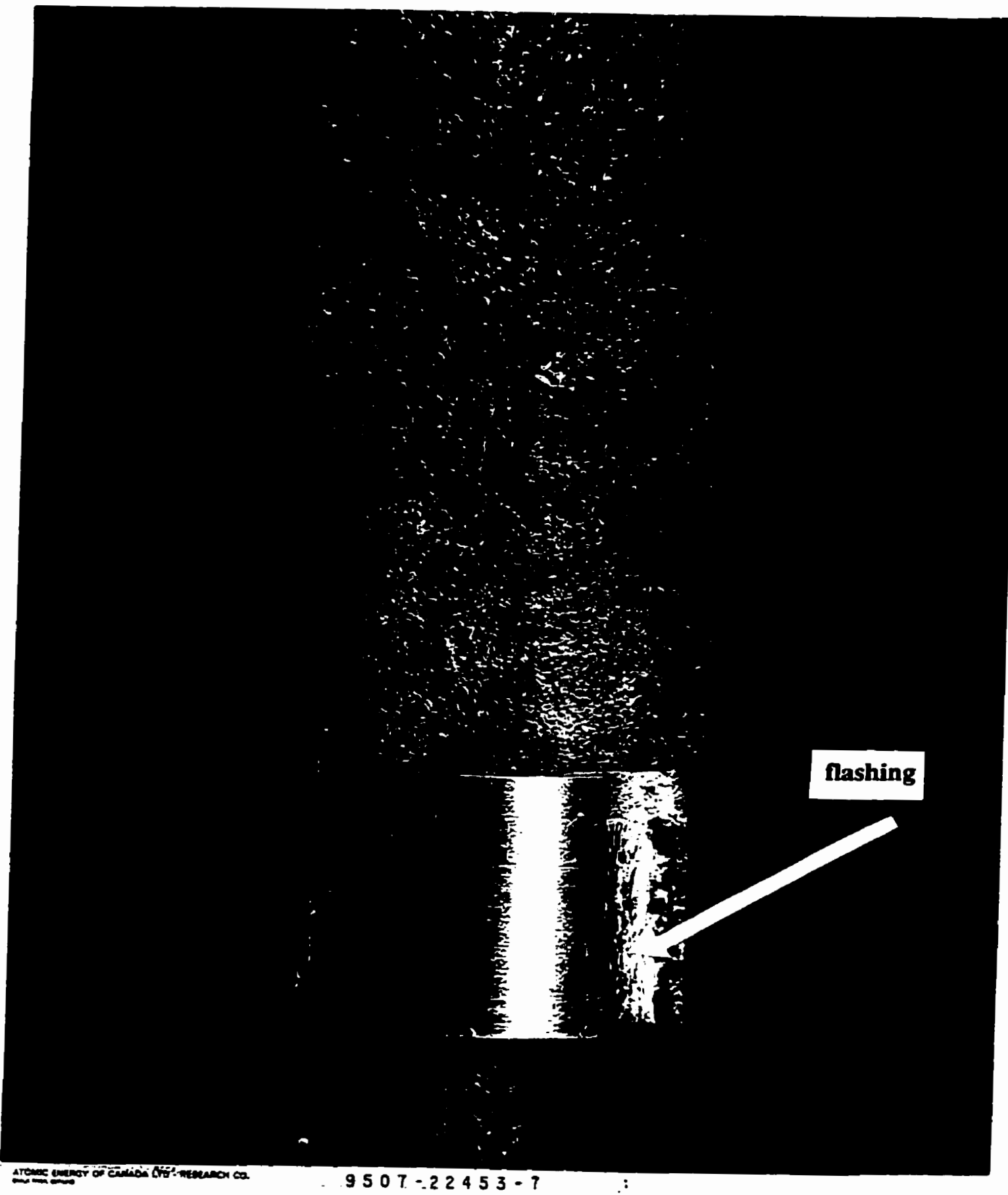


Figure 5.4 (continued)

(c) CANDU-6 broached plate, higher void fraction

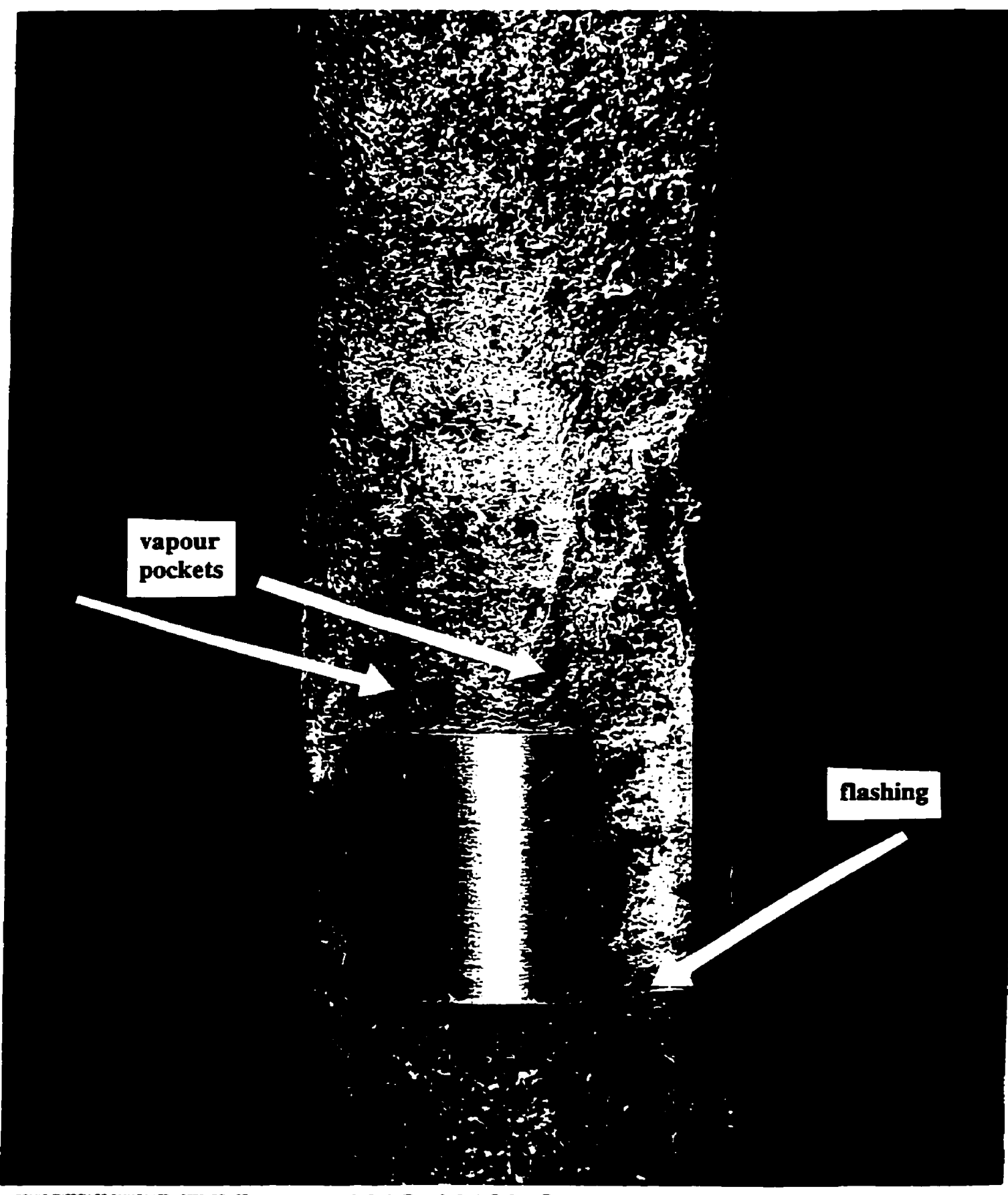


Figure 5.4 (continued)

(d) Darlington lattice bars

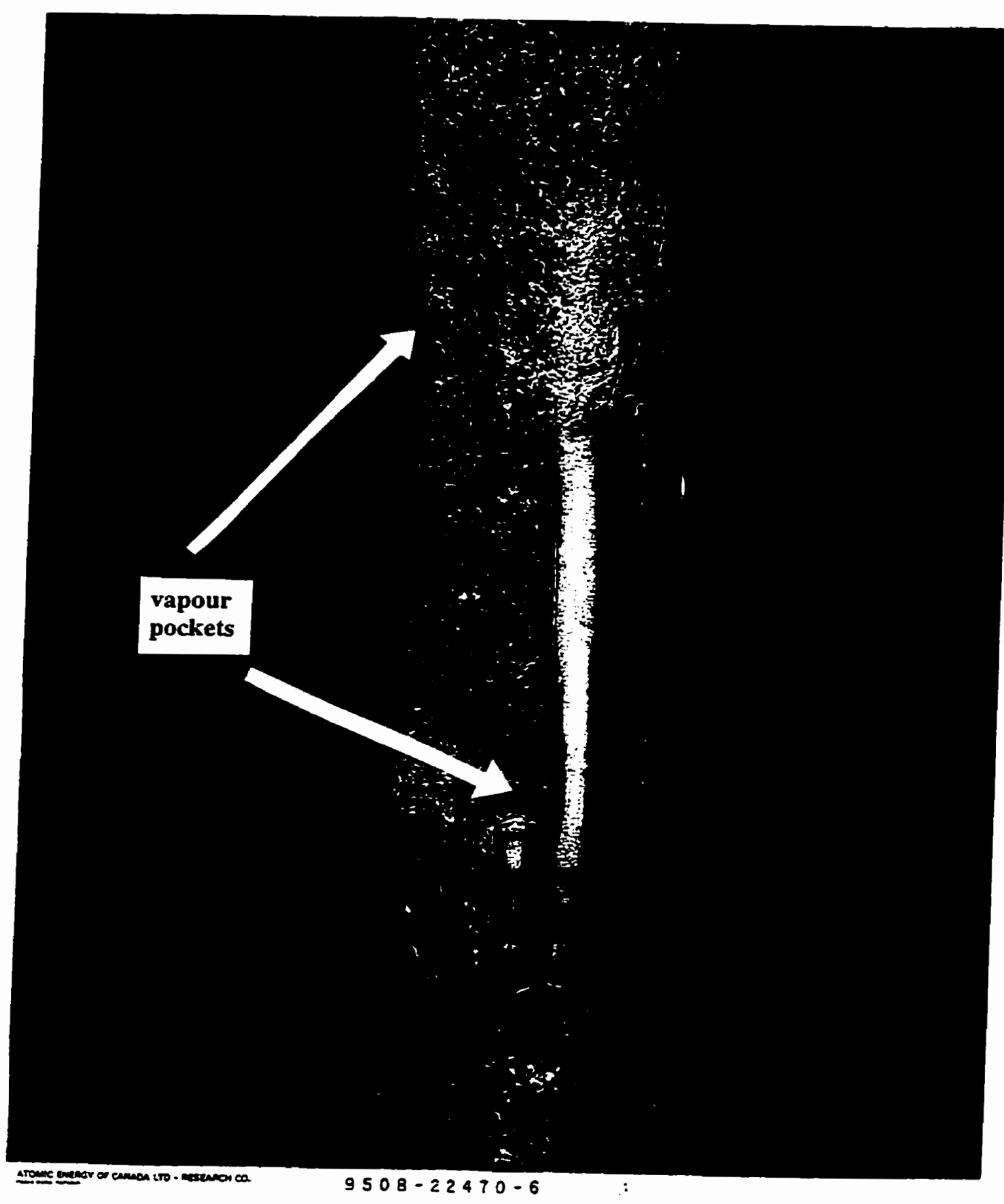


Figure 5.4 (continued)

(e) Darlington lattice bars, high-bar intersection region

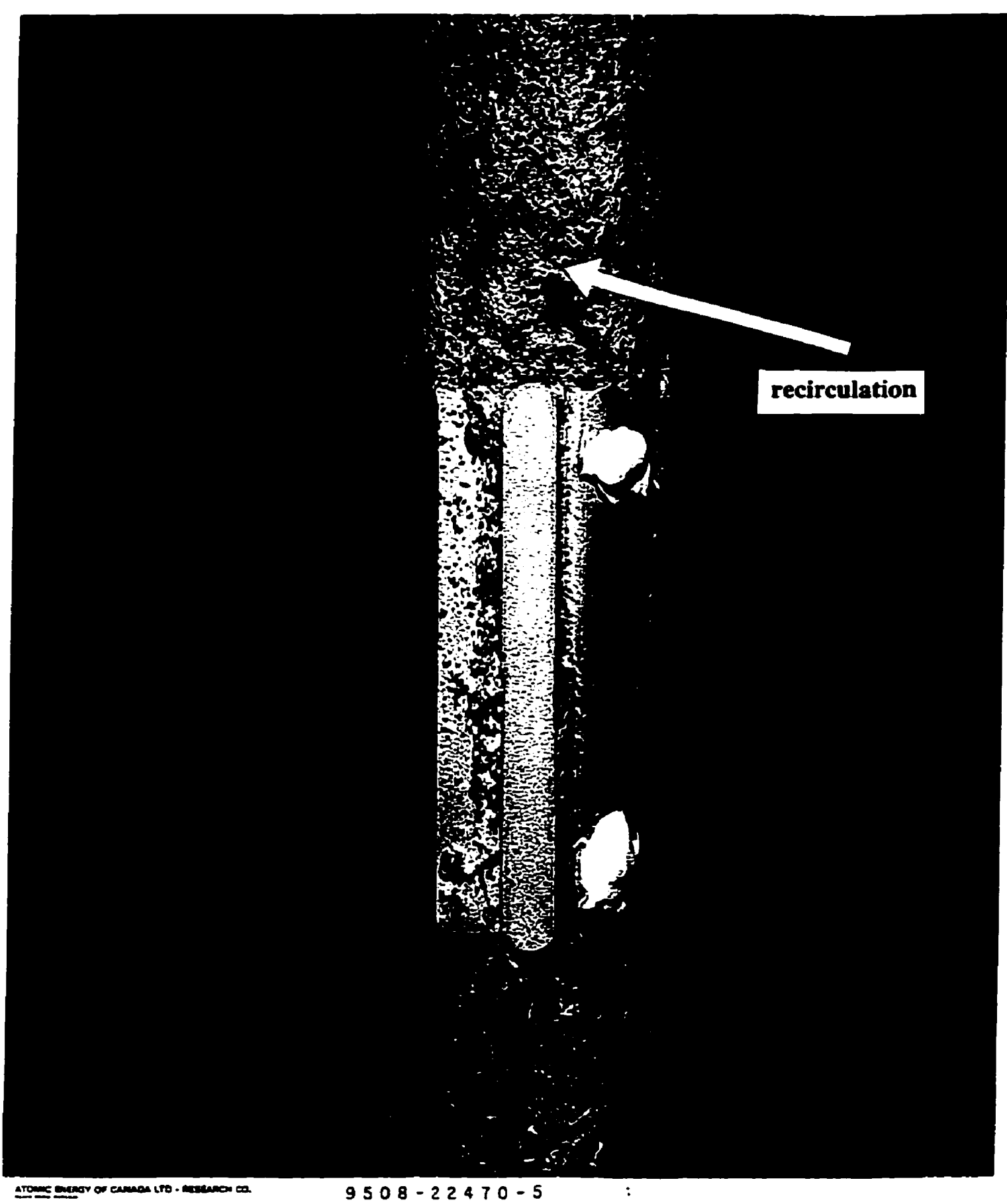


Figure 5.4 (continued)

(f) Wolsong-1 formed bars

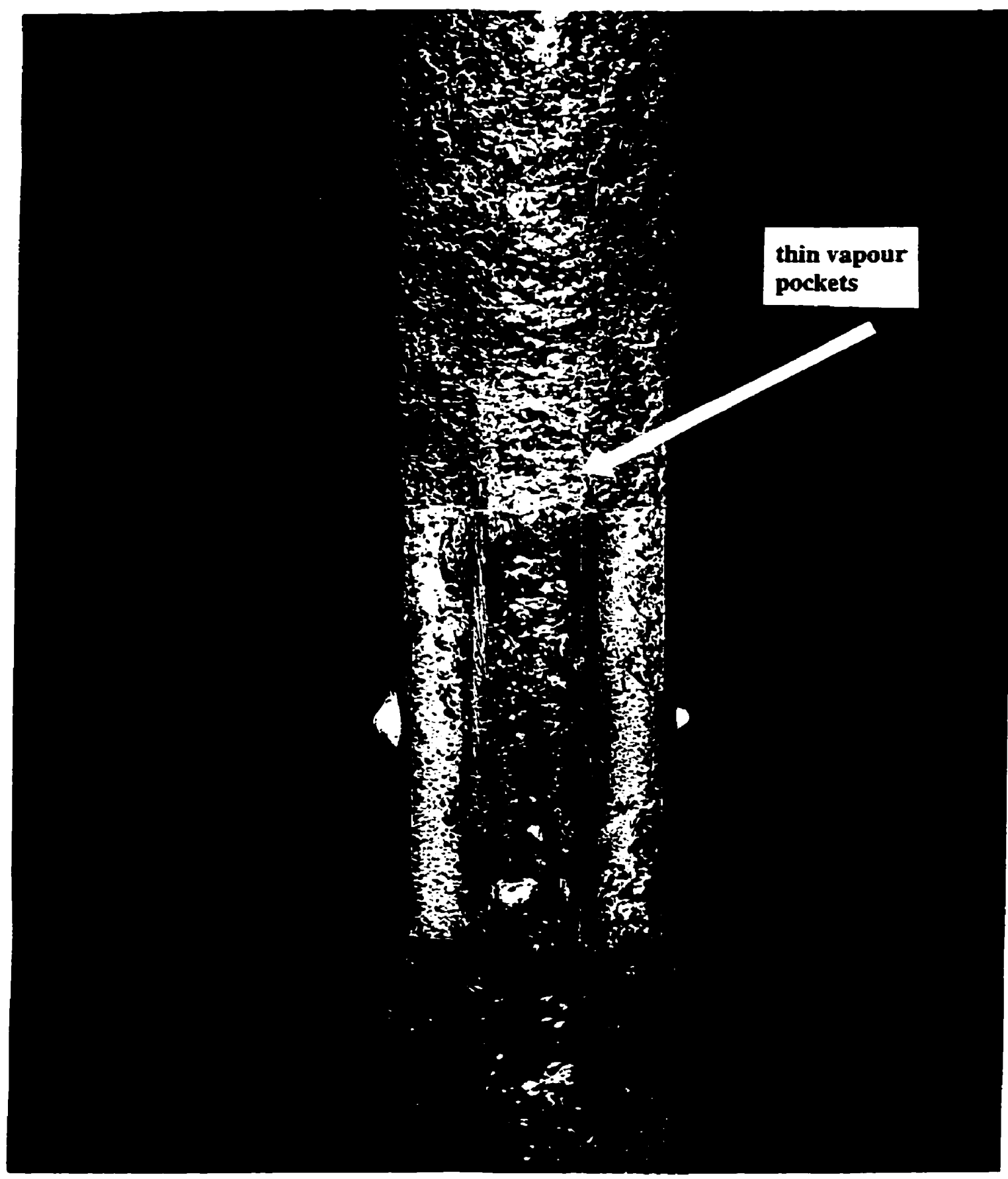


Figure 5.4 (continued)

(g) Wolsong-1 formed bars, intersection region

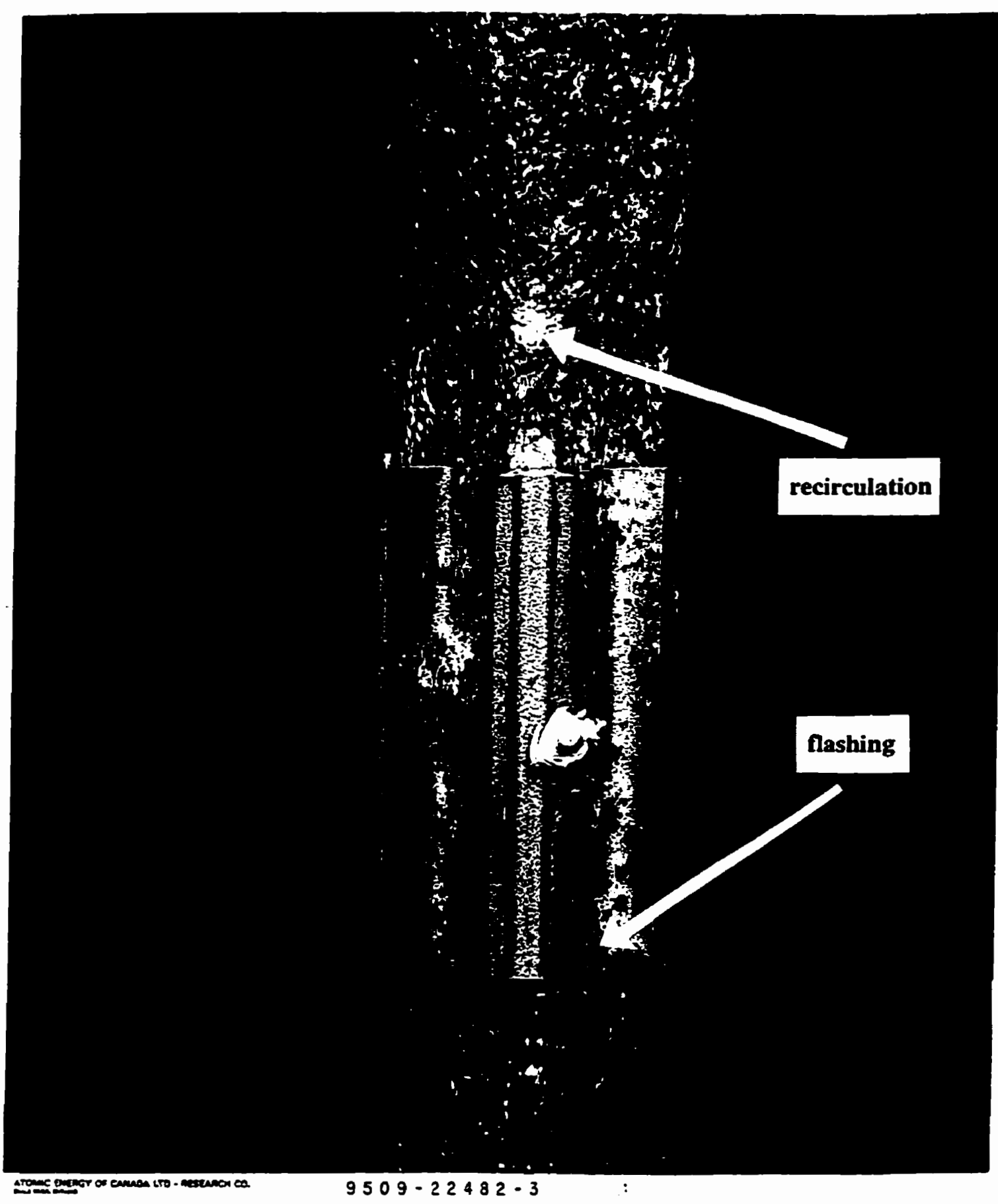


Figure 5.5: Pressure loss apparatus

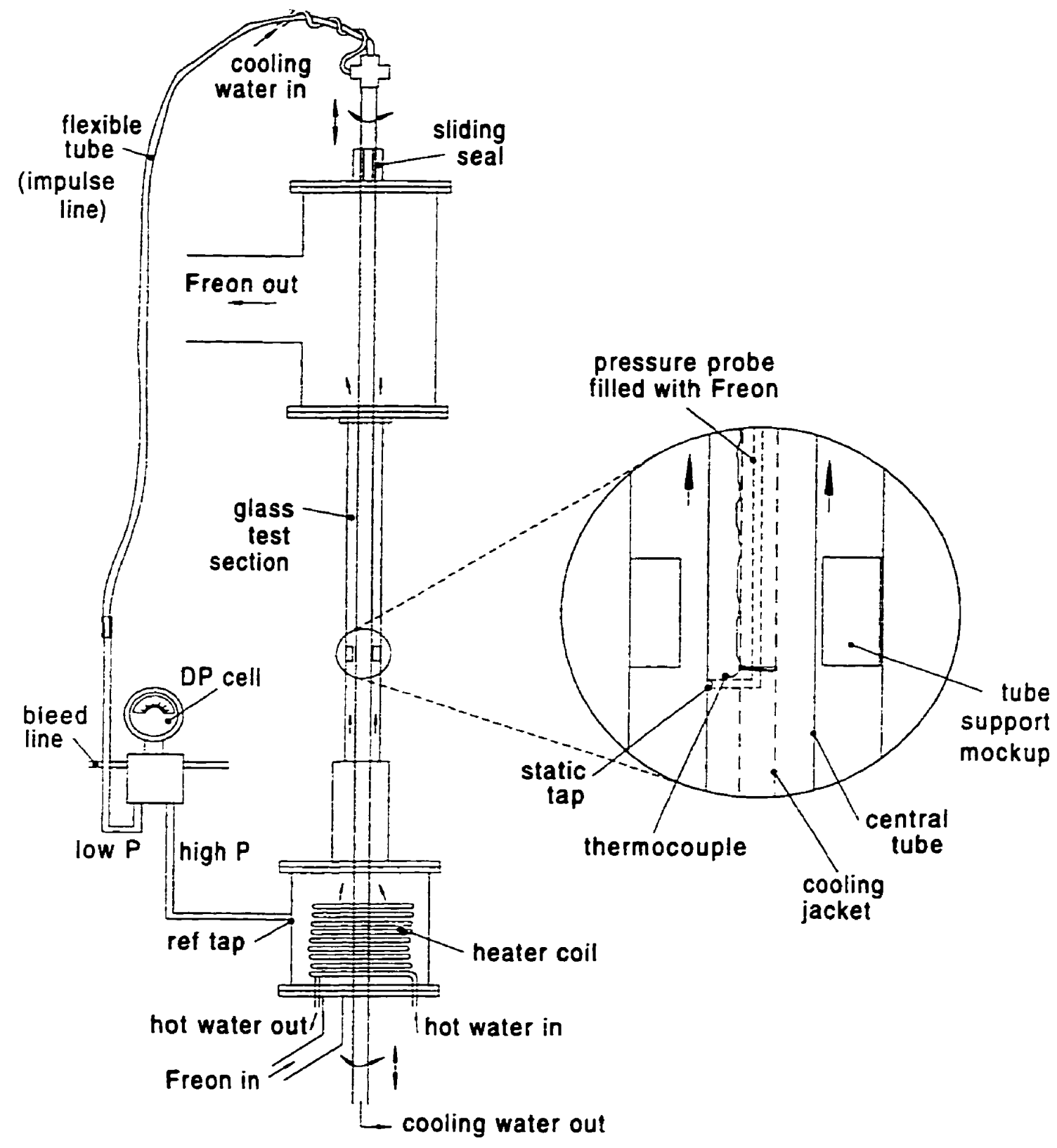


Figure 5.6: Measured axial pressure loss profiles

(a) Bruce broached plate, single-phase (top) and two-phase (bottom)
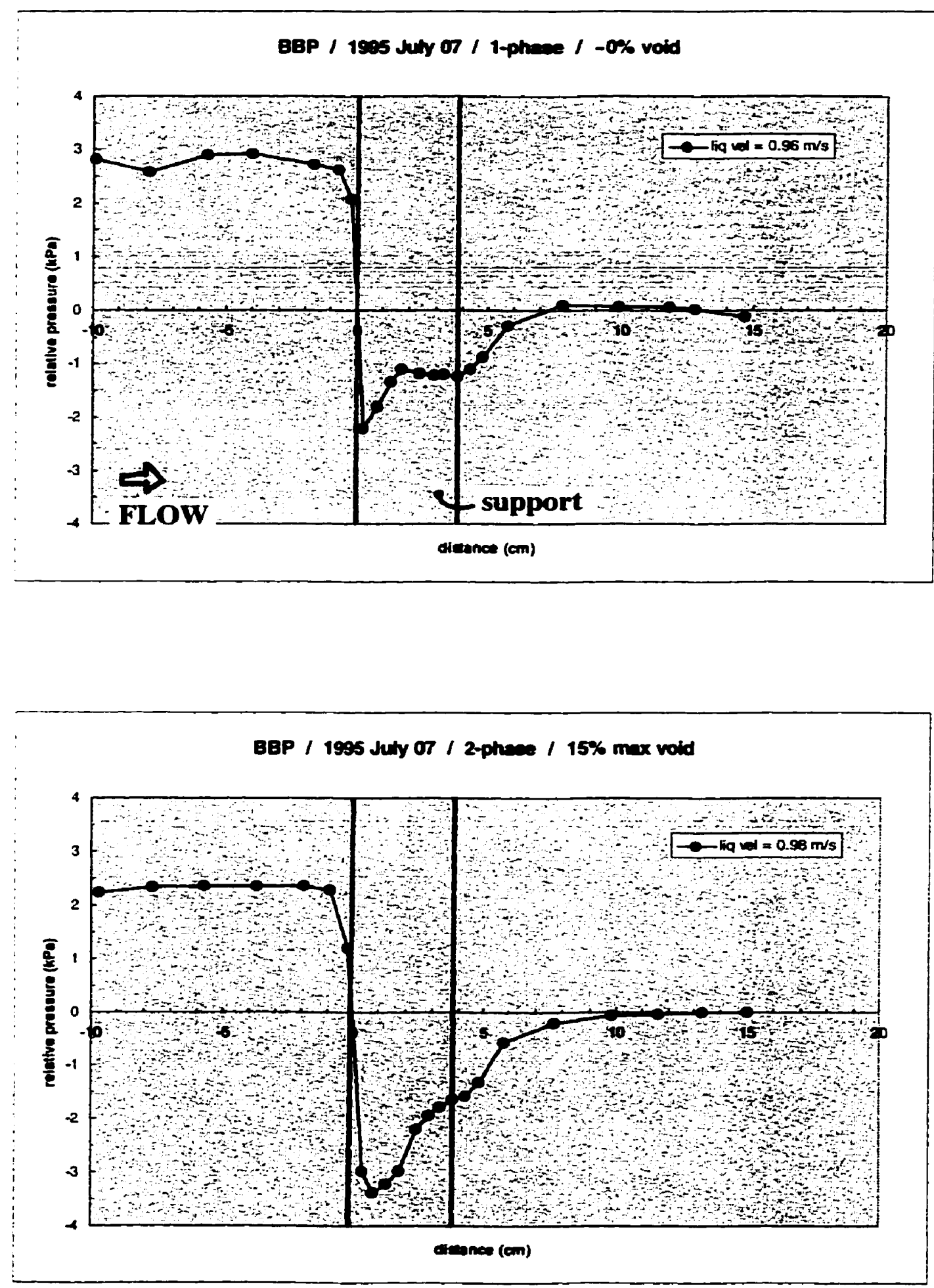
Figure 5.6 (continued)

(b) CANDU-6 broached plate, single-phase (top) and two-phase (bottom)

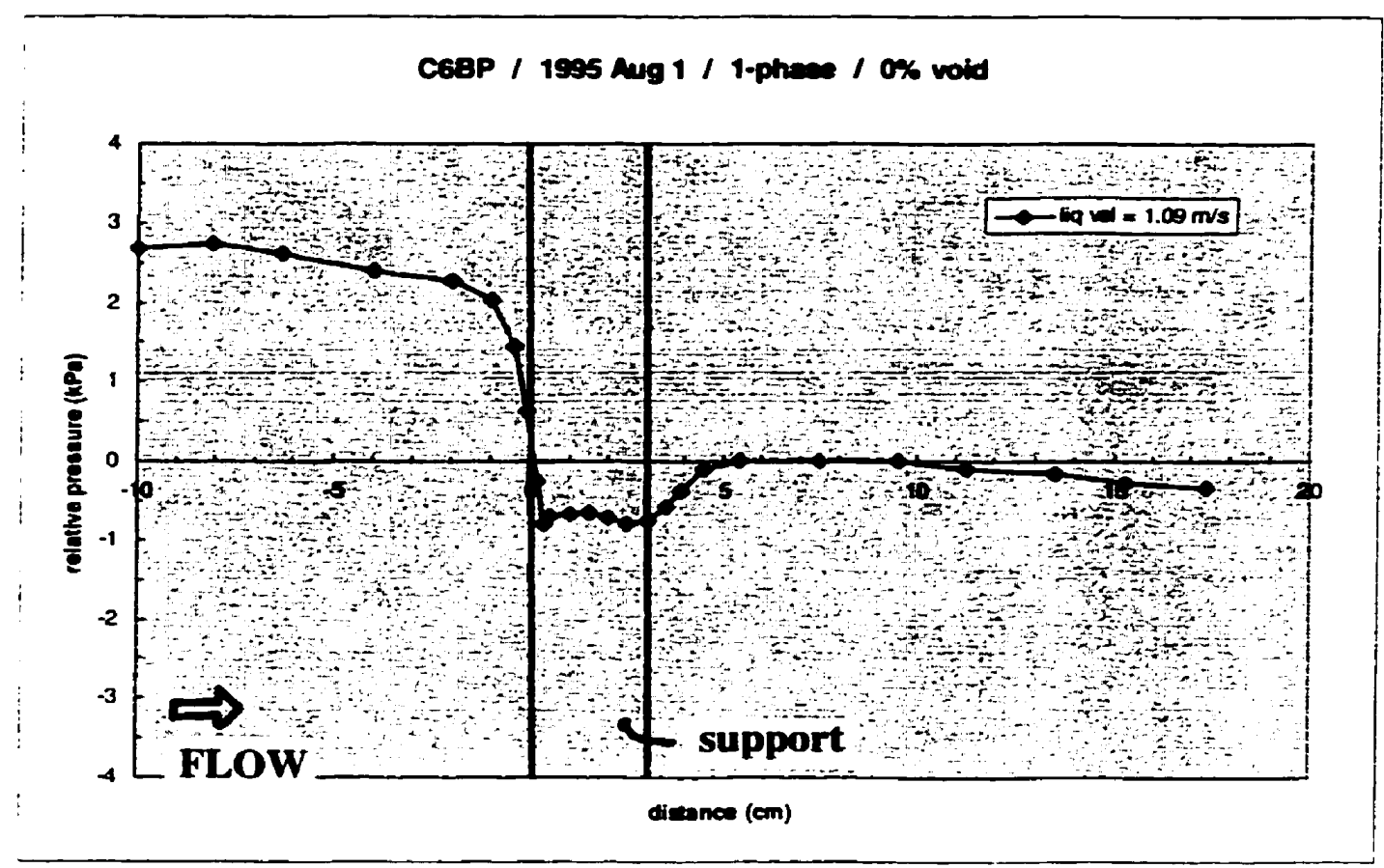

C68p / $1995 \operatorname{Ang} 2$ / 2-phase / $40 \%$ max void

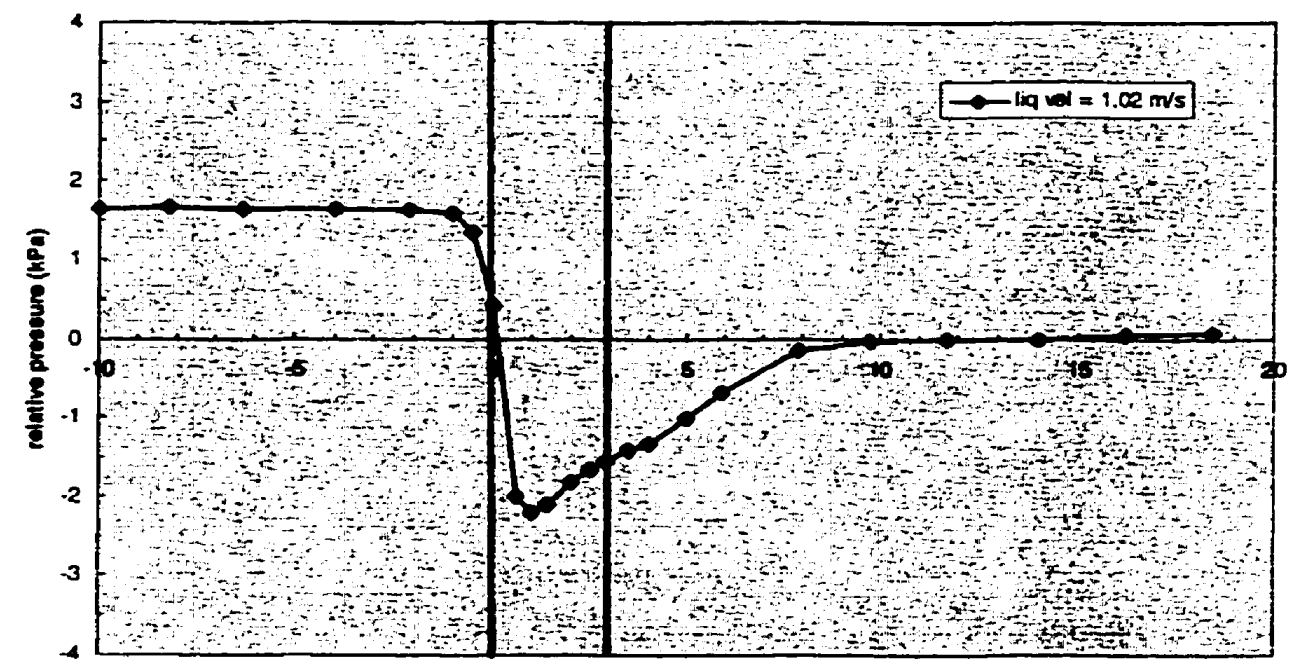

atsinnos (cm) 
Figure 5.6 (continued)

(c) Darlington lattice bars, single-phase (top) and two-phase (bottom)

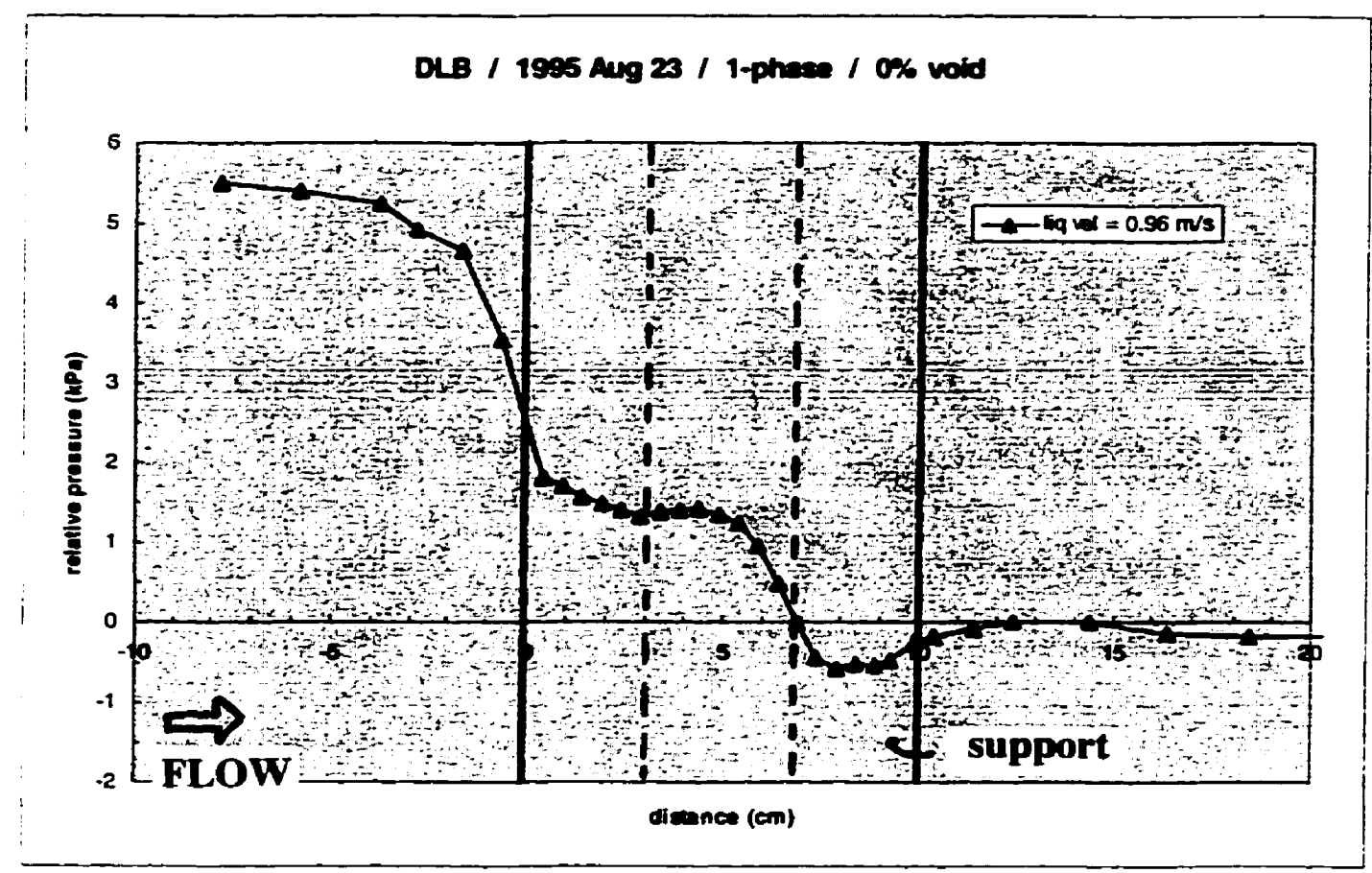

DLB / 1995 Aug 22 / 2-phase / 65\% max void

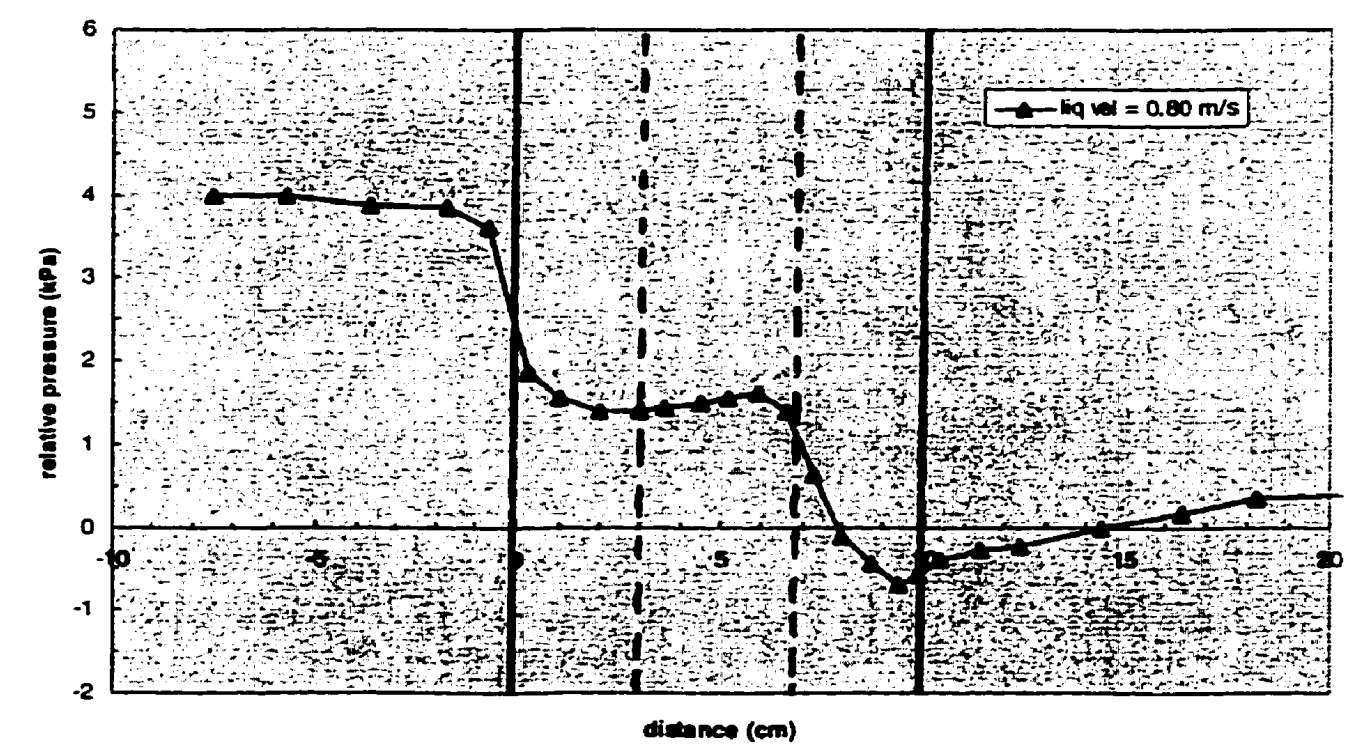


Figure 5.7: Measured and calculated two-phase pressure profile (for Figure 5.6b)

C6BP / 1995 Aug 2 / 2-phase / Run 45

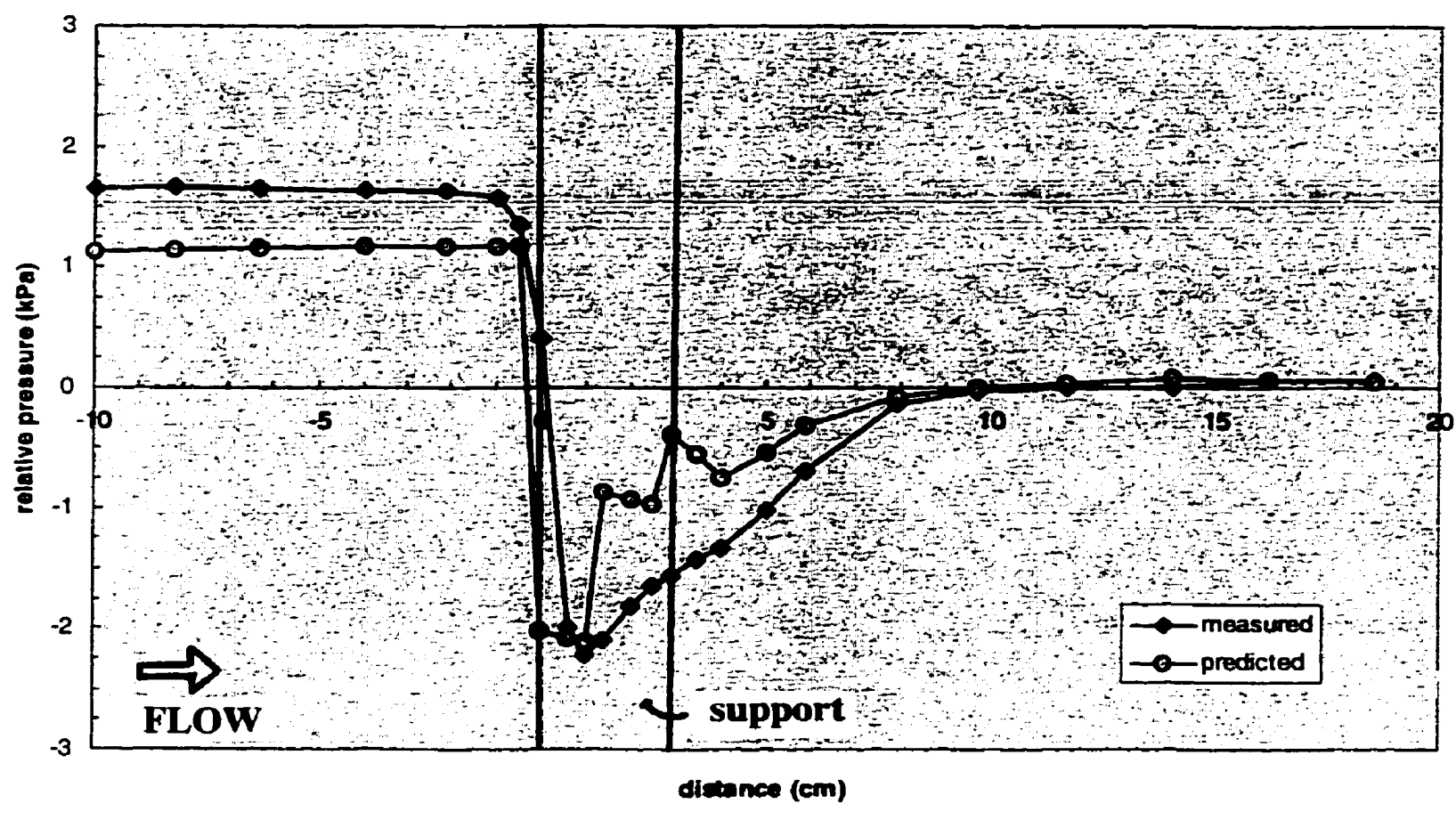


Figure 5.8: Components in overall calculated pressure profile in Figure 5.7

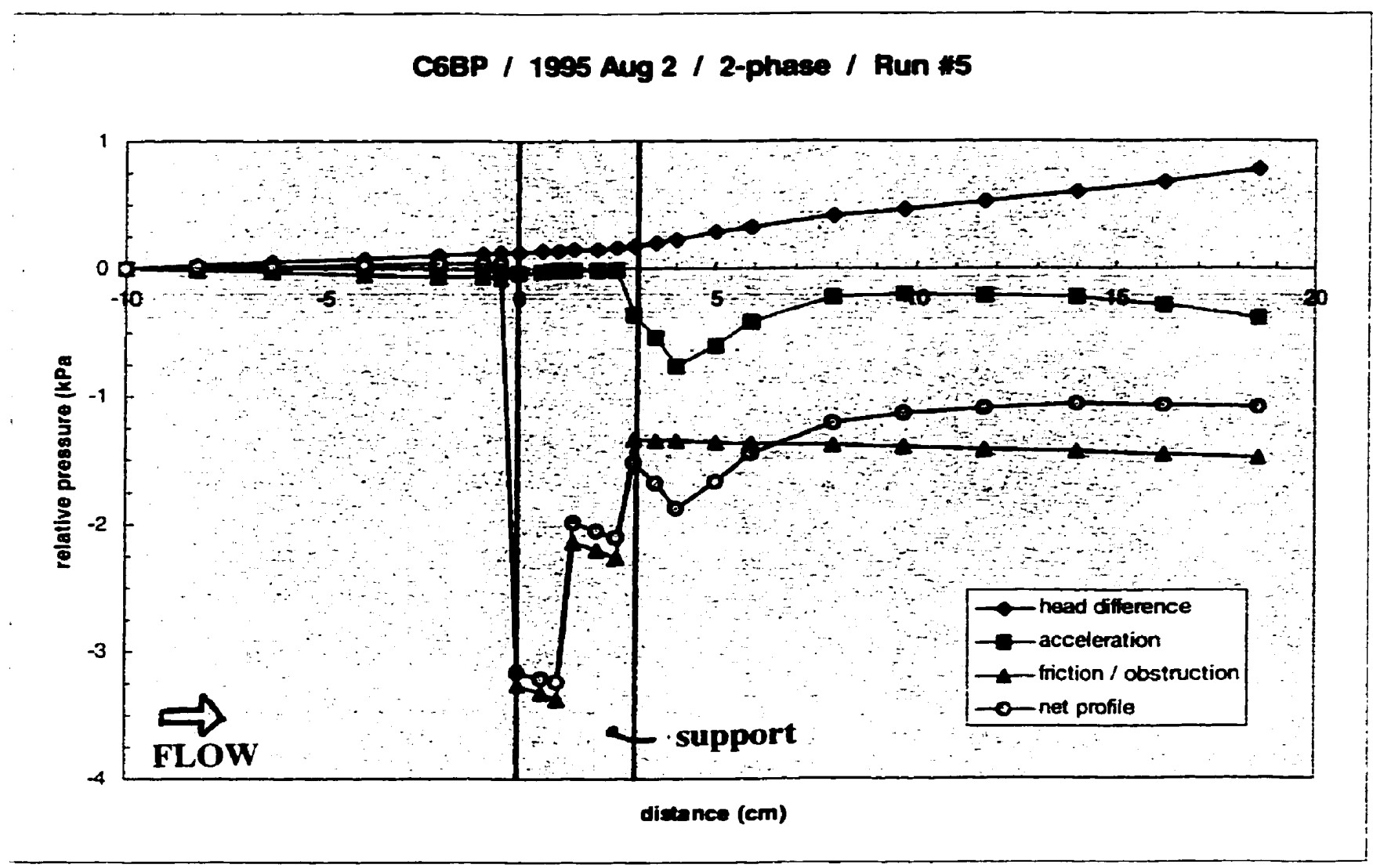

In the above figure, the pressure losses (friction / obstruction and acceleration) were considered negative, while the pressure gains (head difference) were positive. This is opposite to the convention used in Section 7.3.5. 
Figure 5.9: Void fraction apparatus

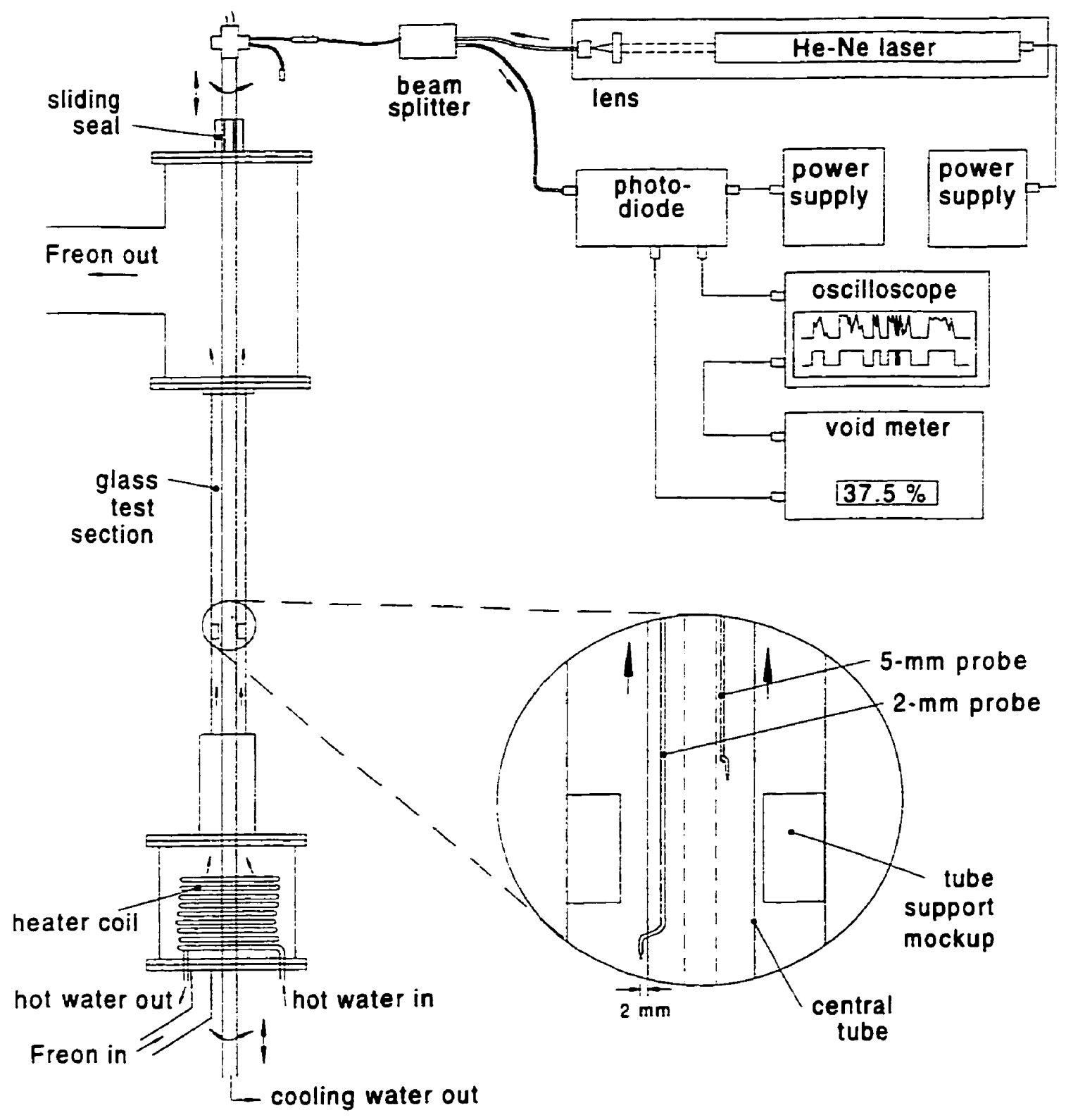


Figure 5.10: Maps of local void fraction - Bruce broached plate

$6 \mathrm{~mm}$ probe - medium void
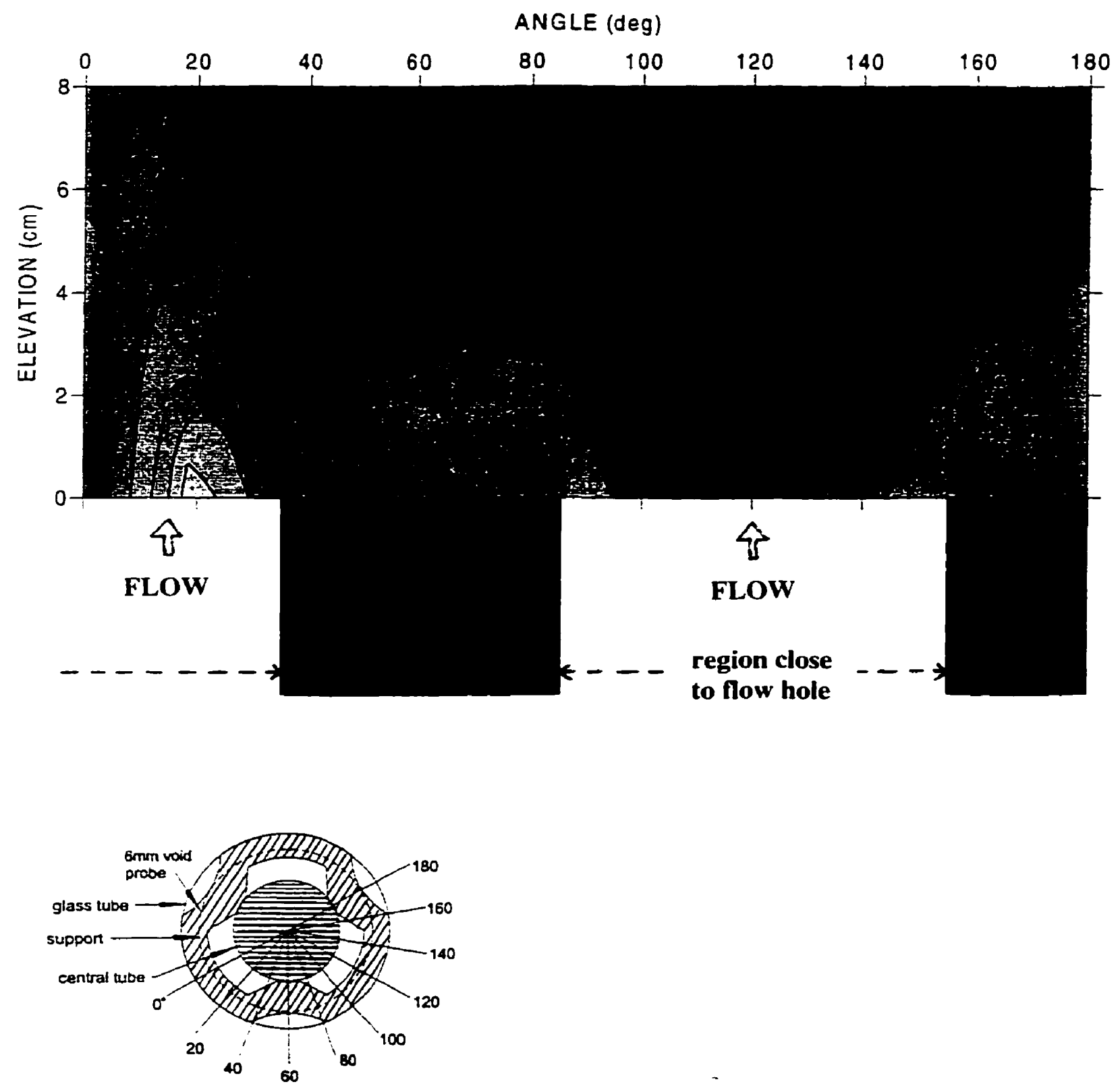
Figure 5.11: Maps of local void fraction - CANDU-6 broached plate

(a) $2 \mathrm{~mm}$ probe - medium void

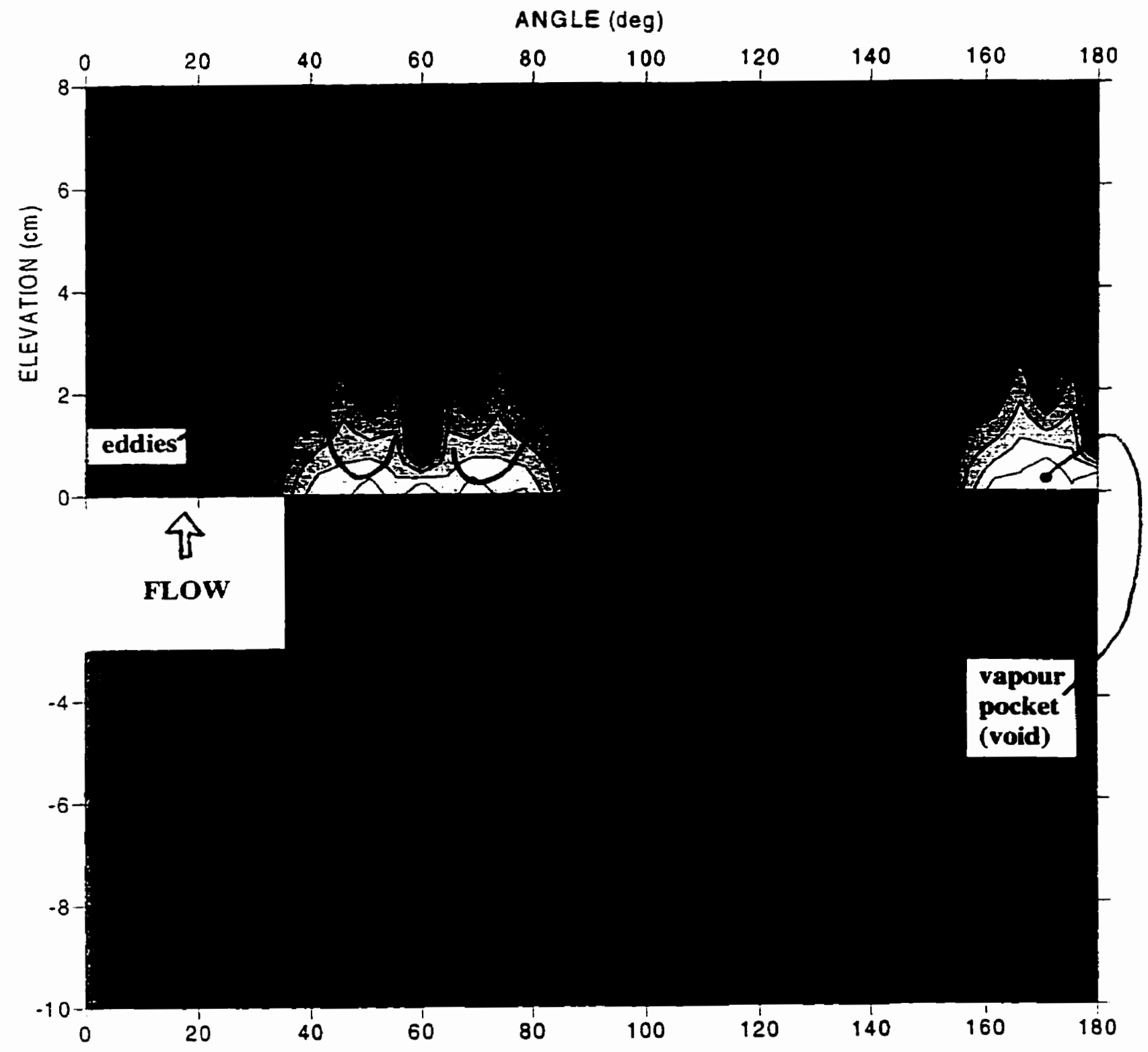


Figure 5.11 (continued)

(b) $5 \mathrm{~mm}$ probe - medium void
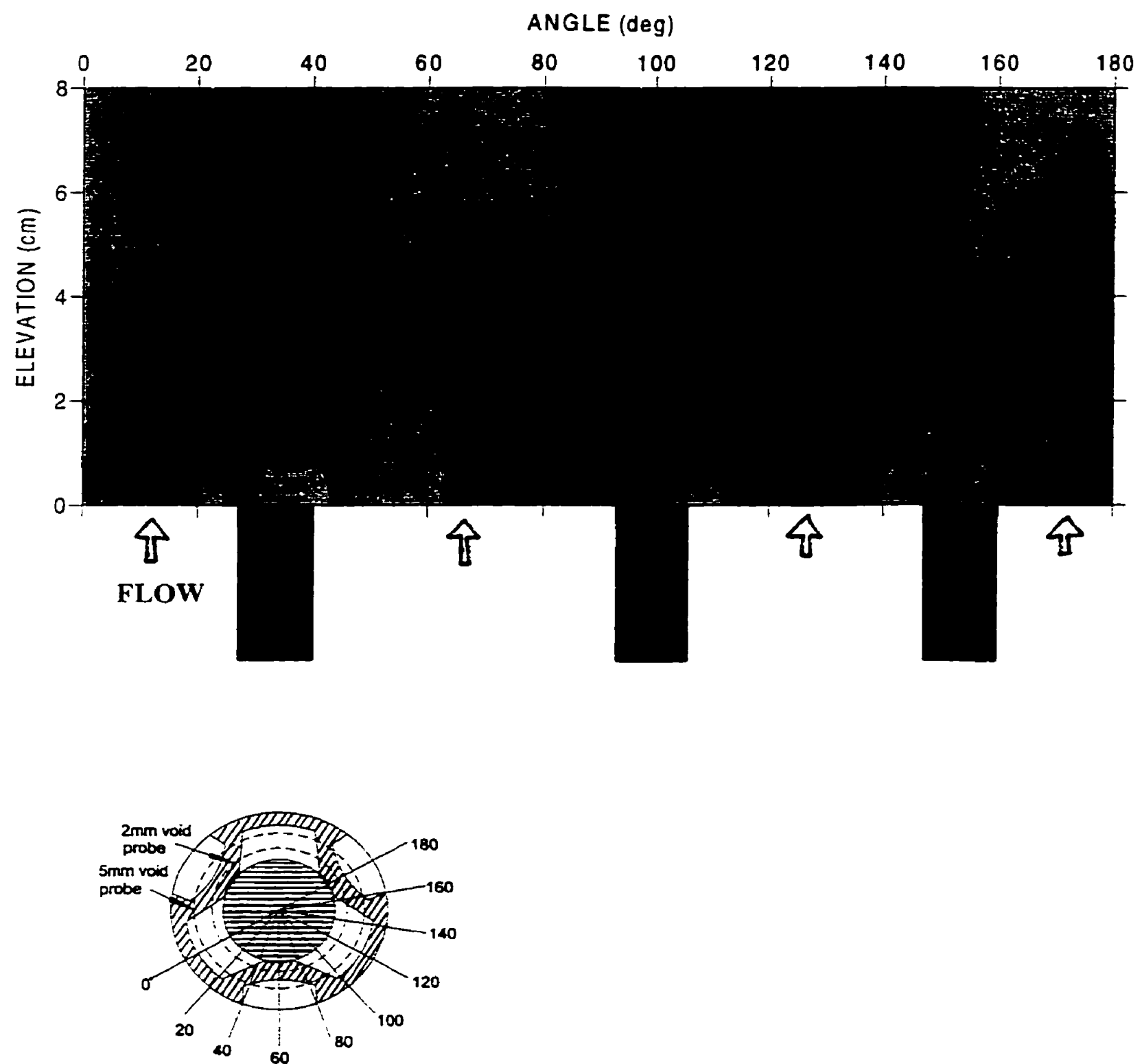
Figure 5.11 (continued)

(c) $2 \mathrm{~mm}$ probe - high void

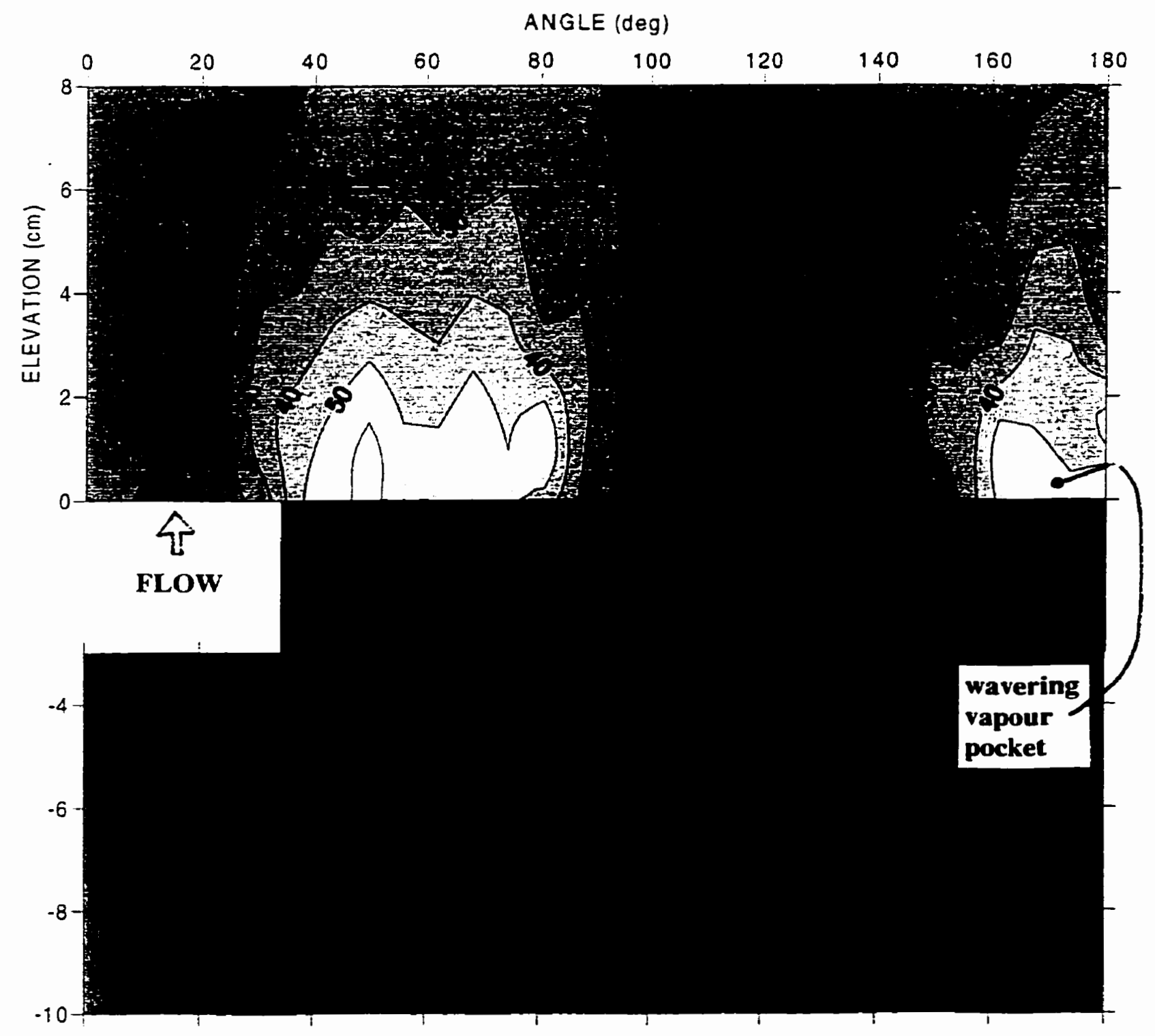


Figure 5.11 (continued)

(d) $5 \mathrm{~mm}$ probe - high void

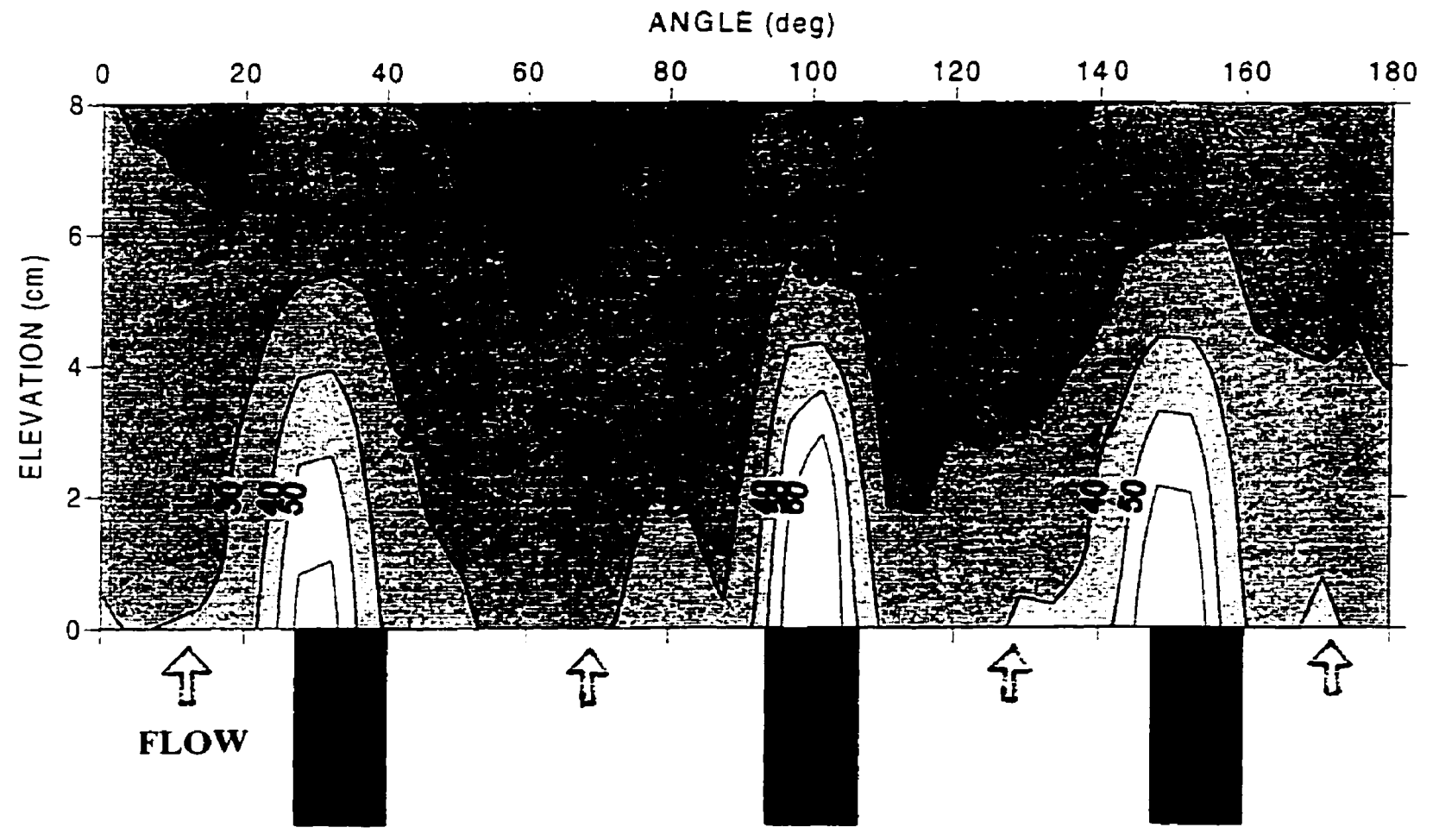


Figure 5.12: Maps of local void fraction - Darlington lattice bars

(a) $2 \mathrm{~mm}$ probe - medium void
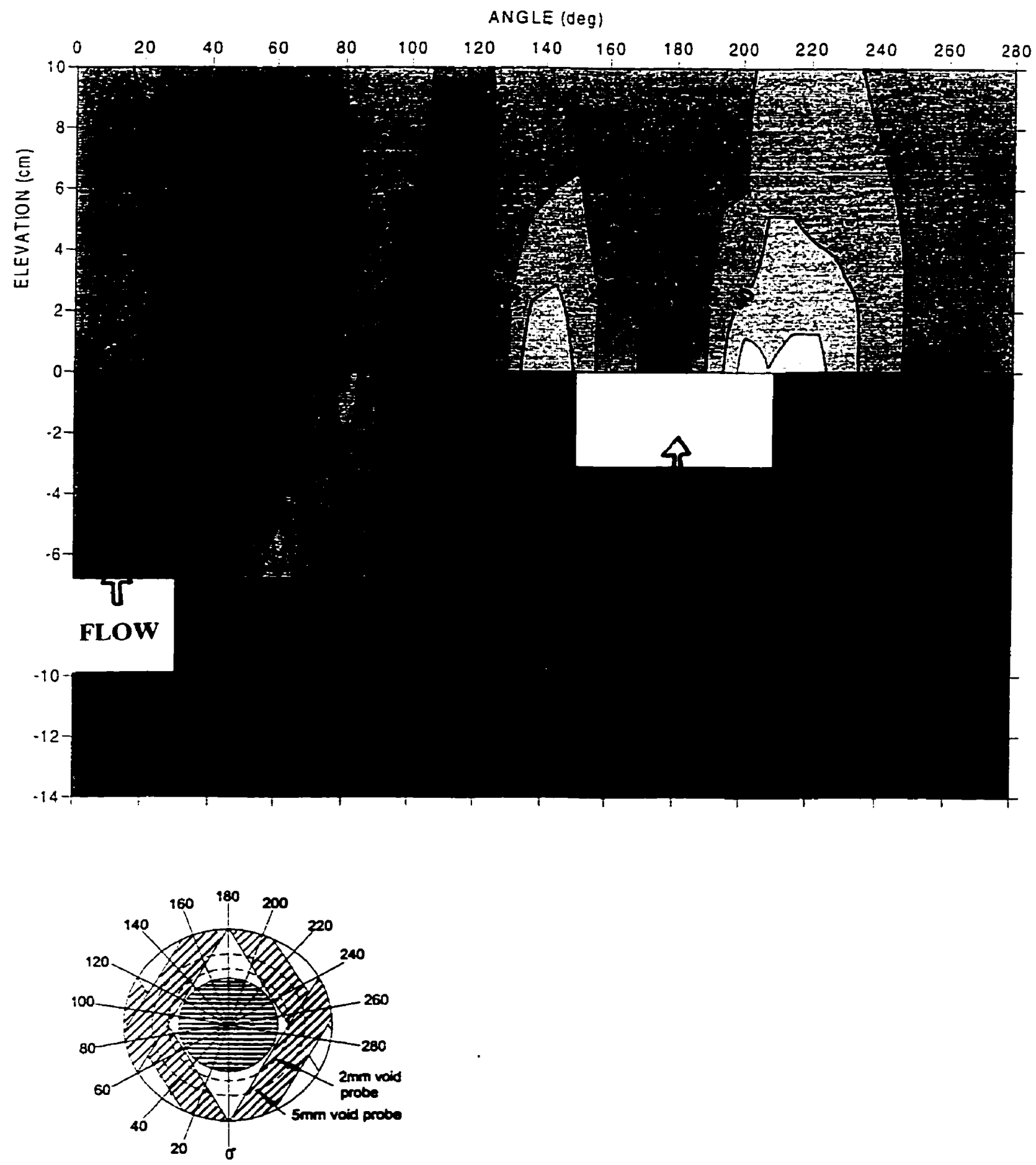
Figure 5.12 (continued)

(b) $5 \mathrm{~mm}$ probe - medium void

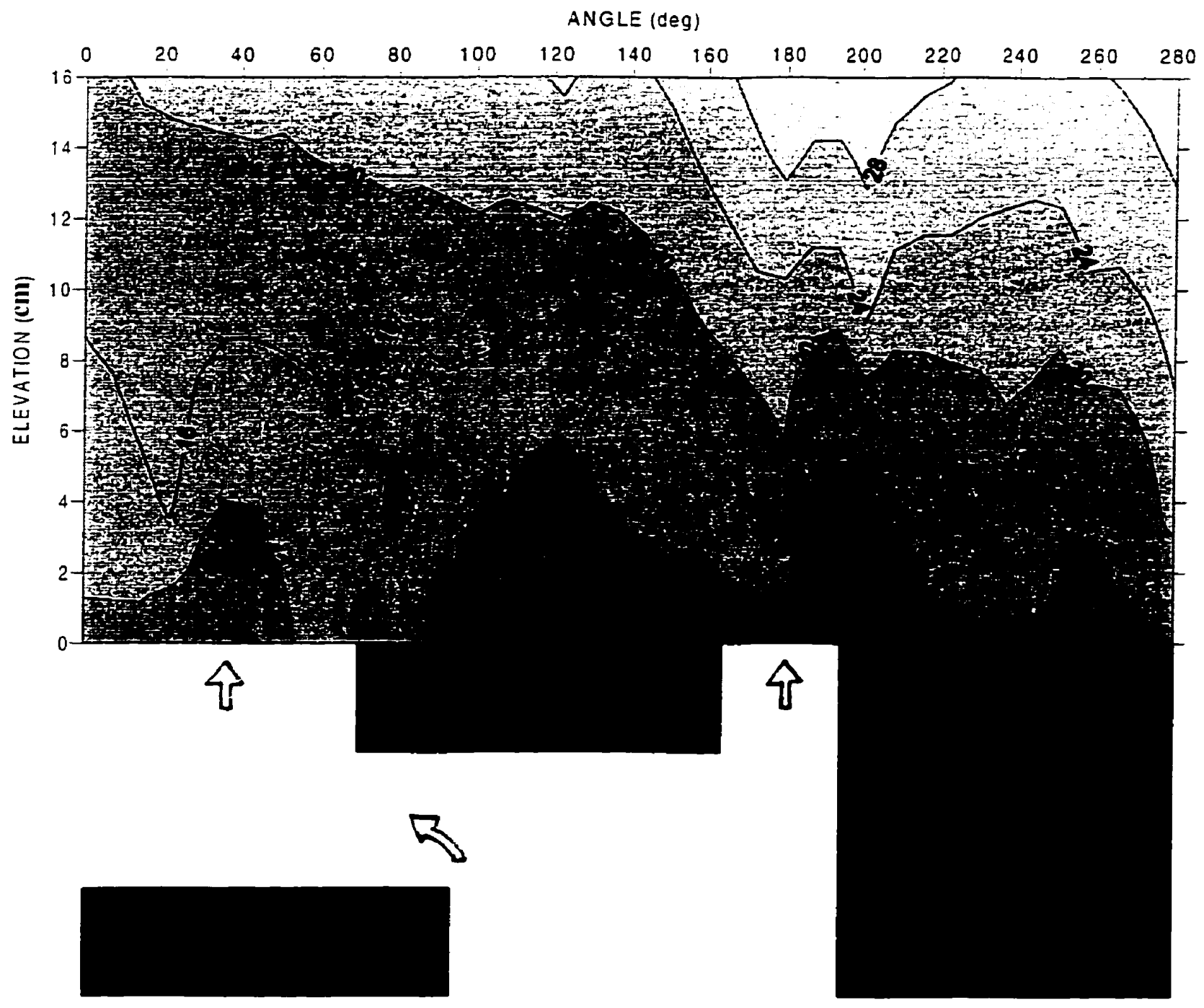


Figure 5.12 (continued)

(c) $2 \mathrm{~mm}$ probe - high void

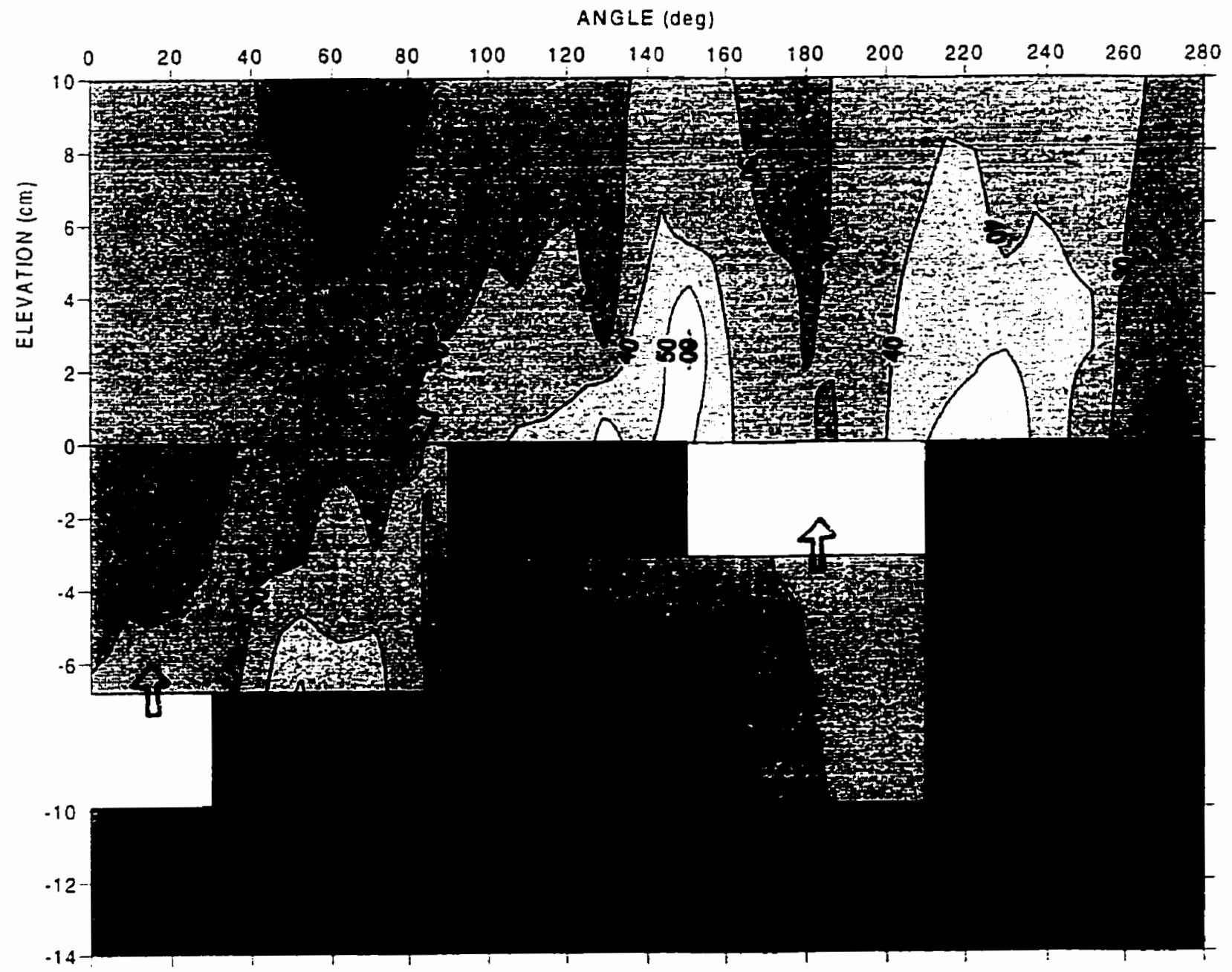


Figure 5.12 (continued)

(d) $5 \mathrm{~mm}$ probe - high void

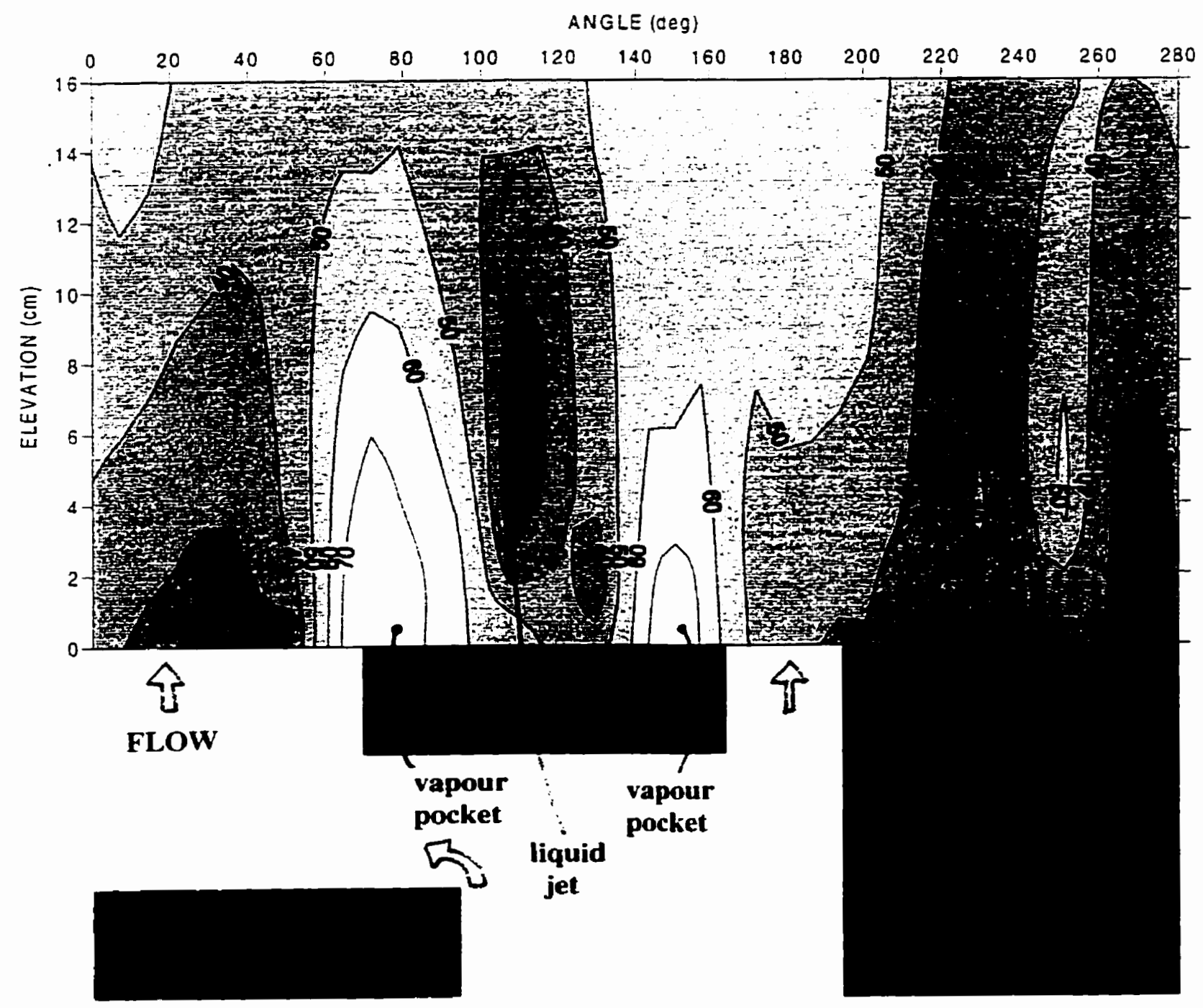


Figure 5.13: Maps of local void fraction - Wolsong-1 formed bars

(a) $2 \mathrm{~mm}$ probe - medium void

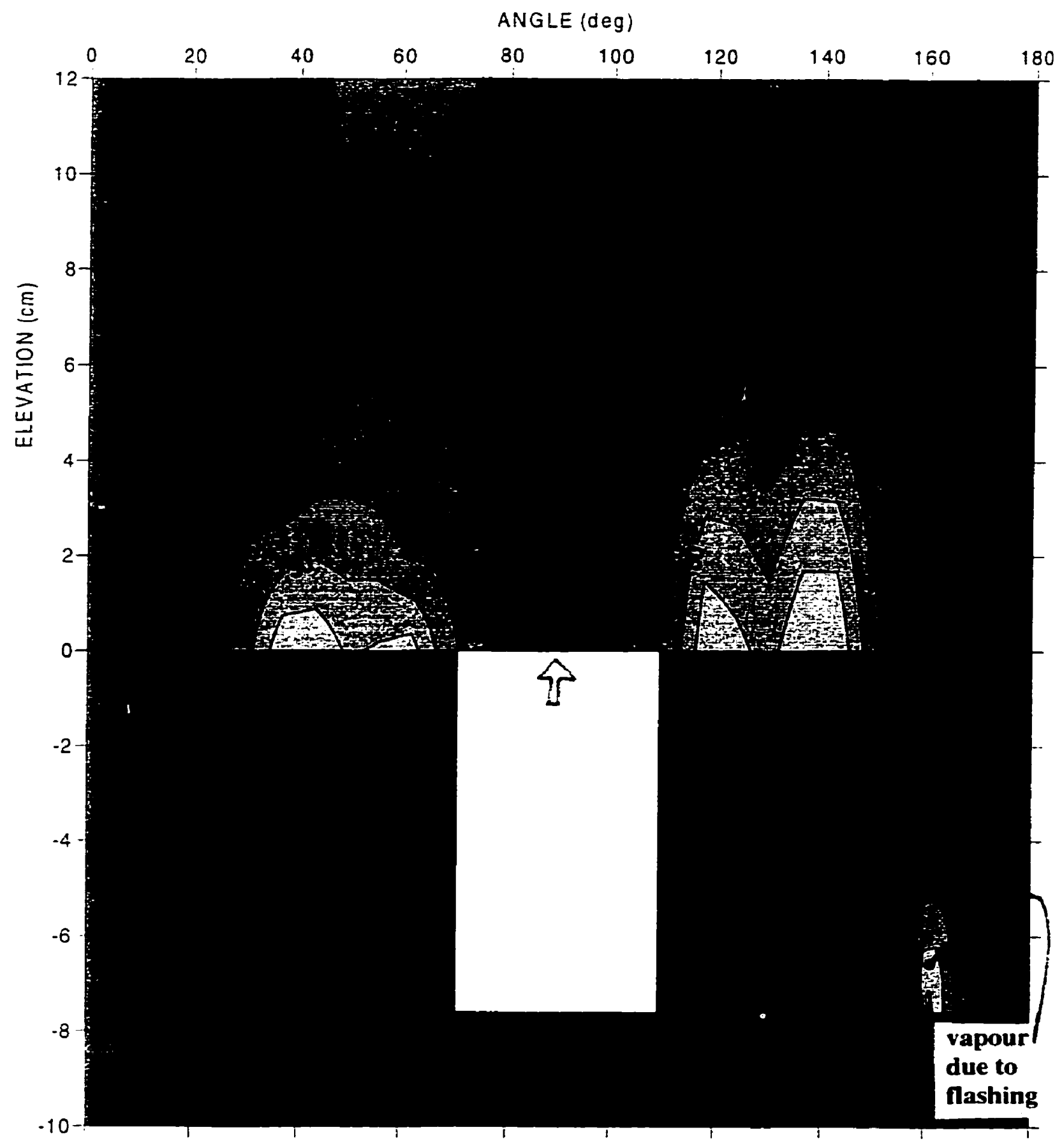


Figure 5.13 (continued)

(b) $5 \mathrm{~mm}$ probe - medium void

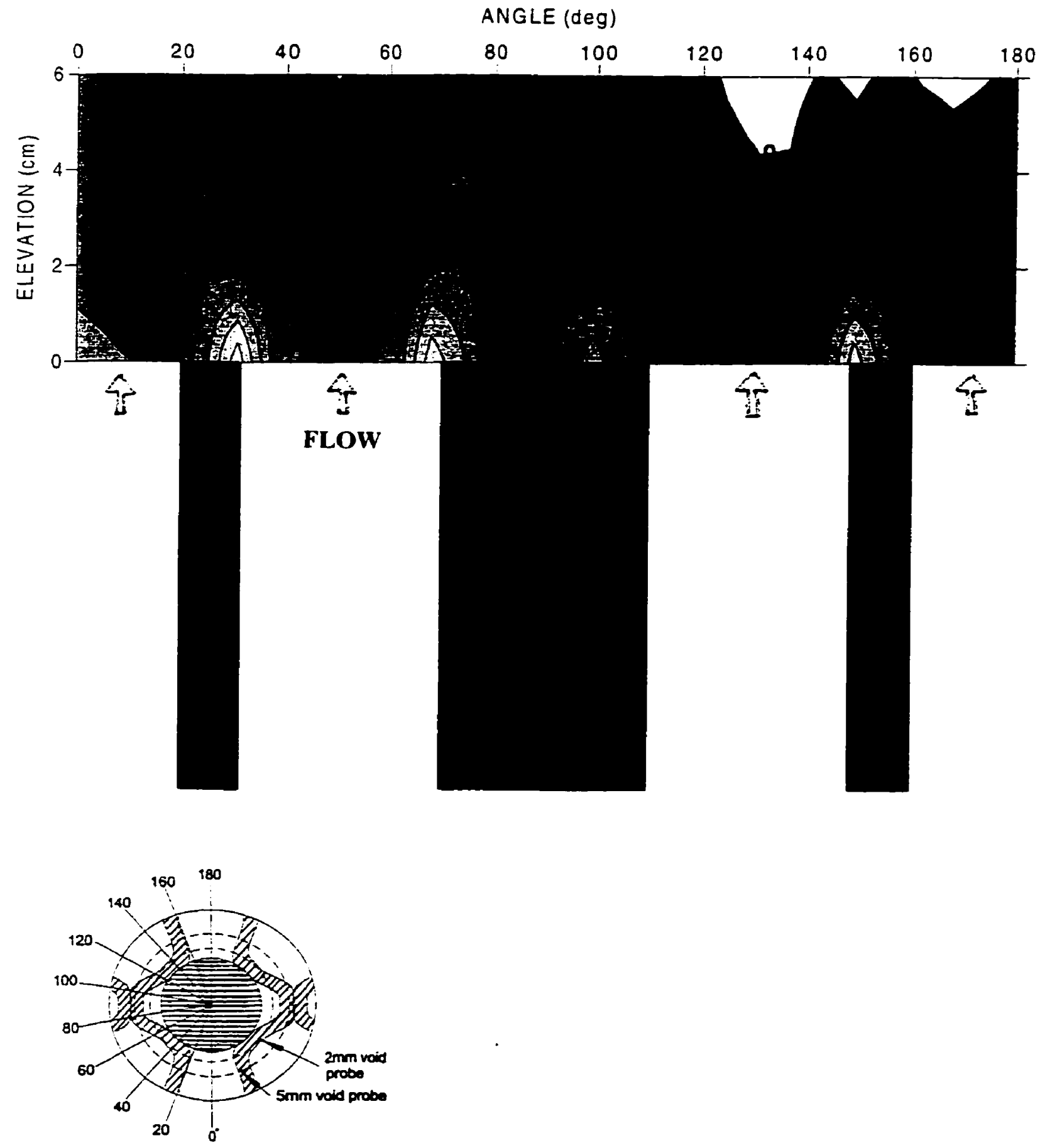


Figure 5.13 (continued)

(c) $2 \mathrm{~mm}$ probe - high void

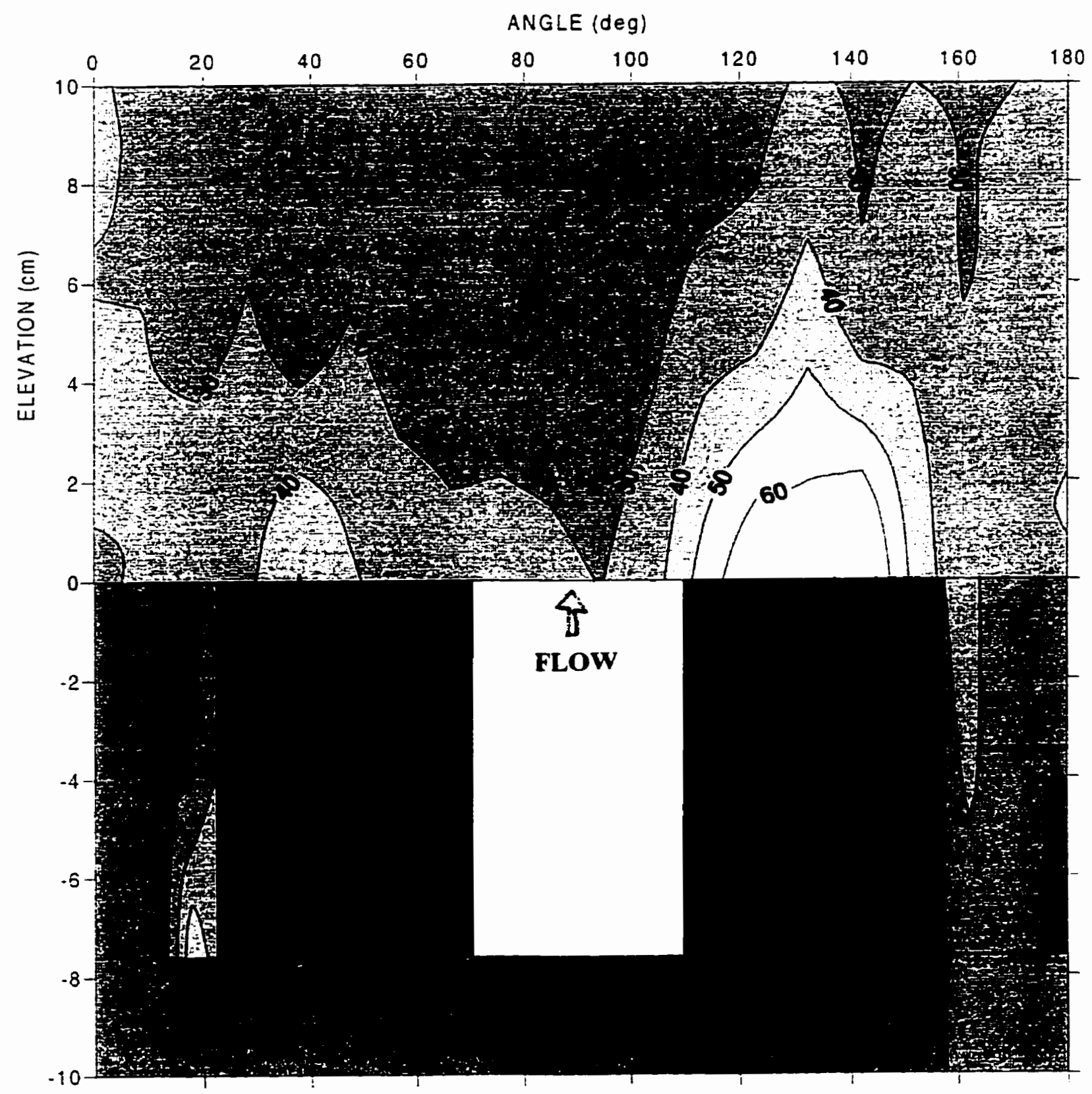


Figure 5.13 (continued)

(d) $5 \mathrm{~mm}$ probe - high void

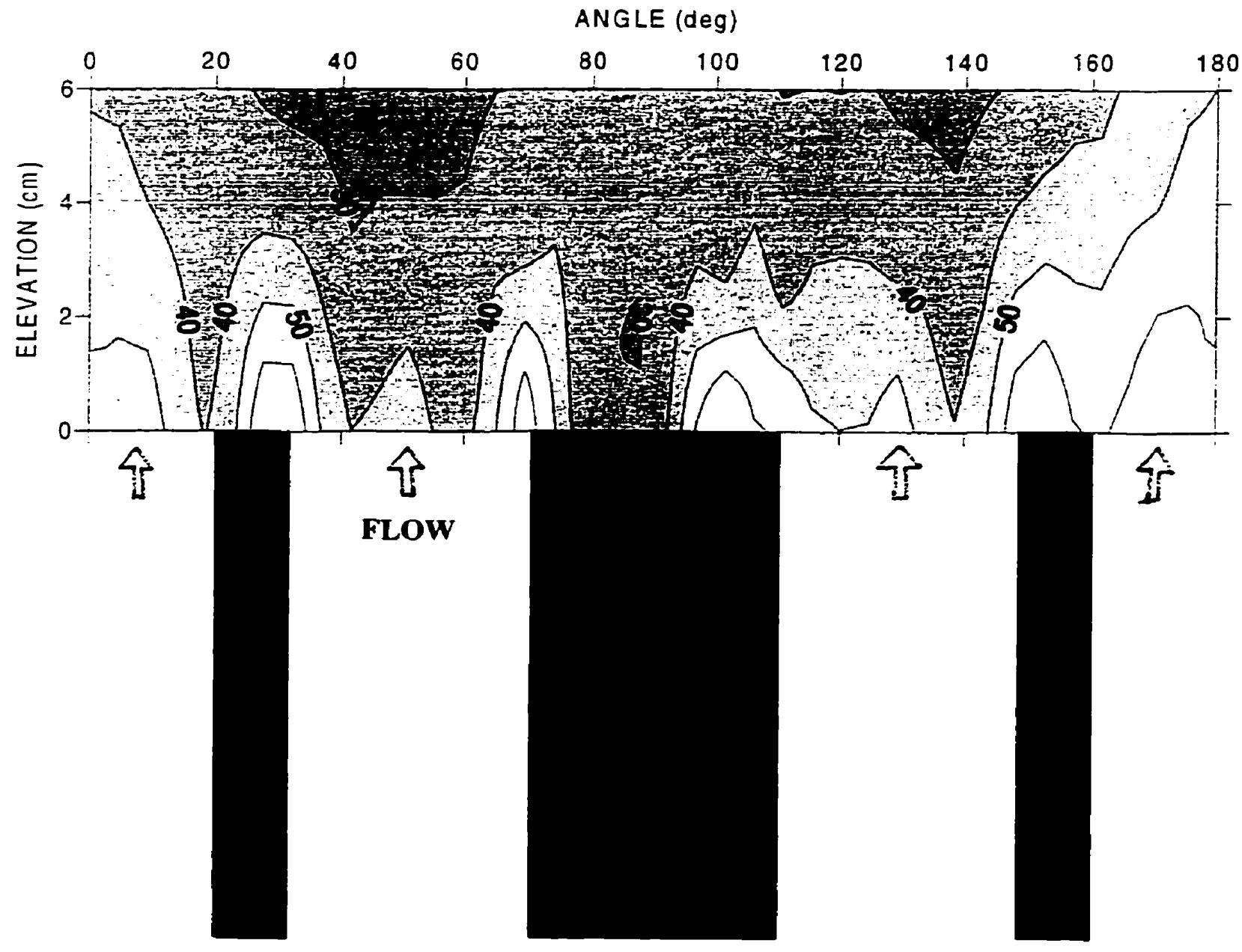


Figure 5.14: Measured axial void fraction profile for CANDU-6 broached plate

\section{C6Bp - 2-phase void profile for DP Run *5}

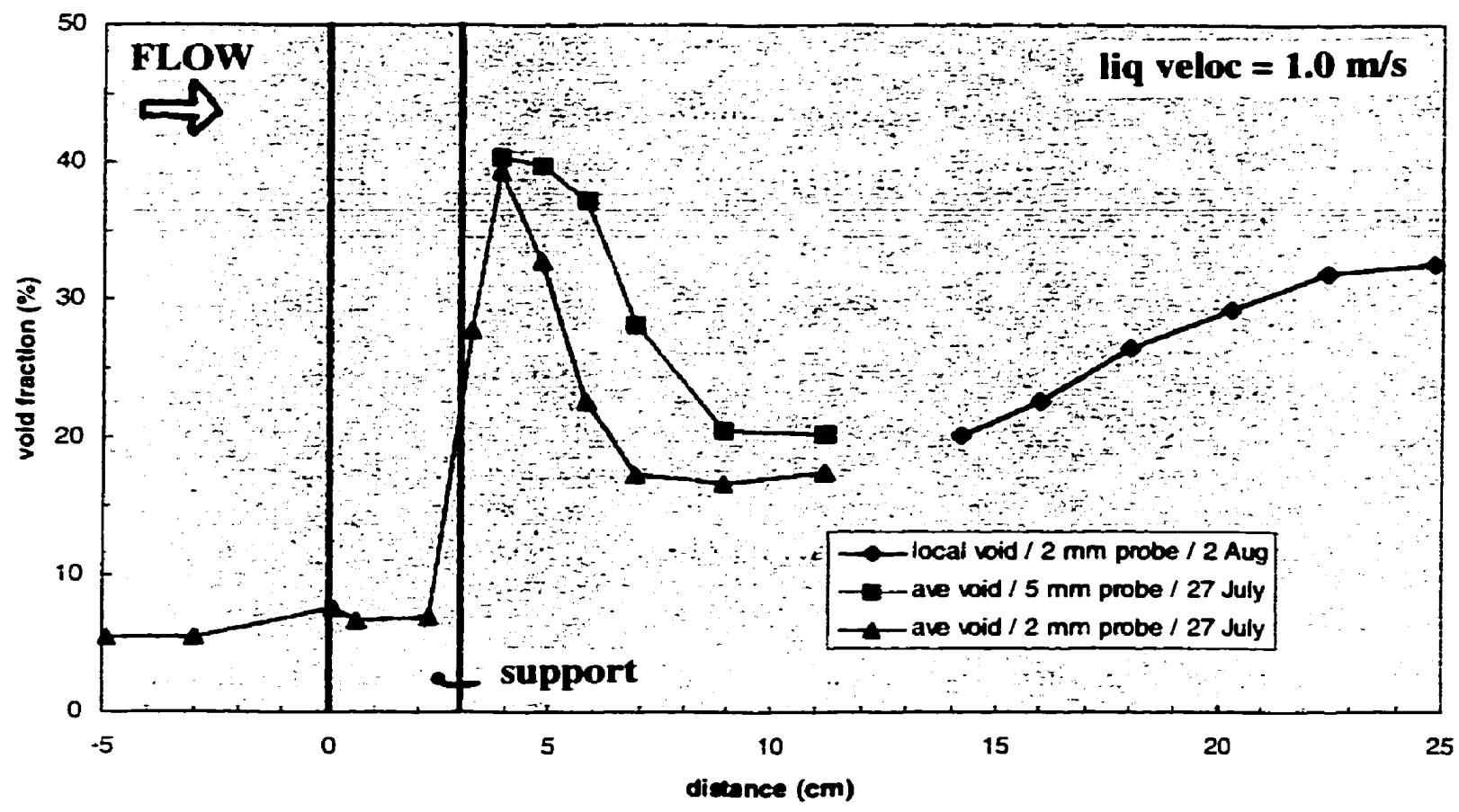




\section{Chapter 6}

\section{CORRELATION BETWEEN FLOW AND FOULING PATTERNS}

Chapter 2 described the appearance and location of deposits on supports in actual SGs. This Chapter endeavors to explain these on the basis of the laboratory tests done in air/water mixtures (Chapter 4) and liquid/vapour mixtures (Chapter 5). The result is a set of proposed fouling mechanisms, which can then be formulated as models and refined using TSFOUL (Chapters 7 to 9).

\subsection{Correlation with Field Data}

\subsubsection{Deposit Lipping}

The very common "lipping" phenomenon observed at the inlet of broach holes can be attributed to the presence of a vena contracta where the mainstream flow separates from the wall following a sharp contraction (as illustrated in Figure 6.1). Such a vena contracta region has been clearly seen and characterized in the flow experiments. It is most pronounced with supports having sharp edges and occupying a significant portion of the flow area, for example, the Bruce-type broached plate. The flow separation is accompanied by a marked and sudden drop in pressure at the inlet along a distance of the order of a few millimeters, which is consistent with the extent of the observed lips.

In the vena contracta region, a low-velocity recirculation zone is created beside the support, in which a tiny deposit can begin to grow. The growth of this deposit can be encouraged further by a consolidation process. The sudden pressure decrease will cause a thermally-saturated liquid in the bulk fluid to flash into vapour. This, in turn, allows solubles such as iron and copper to precipitate from the liquid, to enter the pores of an existing deposit, and to harden this deposit. Deposition followed by such consolidation 
may explain the presence in some SGs of matching lips of deposit growing out from the tube toward the plate at the inlet of the support, in spite of the tube surface itself not featuring a region of flow separation.

The amorphous clumps observed in the fouling-loop deposit (see Figure 2.3) are evidence of precipitation of solubles, because consolidation by solubles such as iron or copper leaves amorphous deposits (as opposed to agglomerates of particles or crystals). The consolidated deposit is also typically very hard. Such consolidation is believed to be responsible - at least in part-for the very hard deposits in the Bruce A SG (see Section 2.1.3), which had a mixed iron/copper feed-train.

As the deposit grows, the flow contraction and associated boundary-layer separation will become more severe. This brings forth the possibility of an auto-catalytic behaviour in which the fouling becomes progressively worse. However, the flow through that support channel will then progressively decrease, either due to flow diversion to other channels or due to the reduced overall recirculation flow rate in the blocked SG. Such complex changes in deposit growth rate were investigated using TSFOUL, as described in Chapters 9 and 10.

Lips have also been found at the outlet of broach holes. This phenomenon could be related to the presence of quasi-stagnant vapour pockets in two-phase flow which encourage particles to settle on the downstream side of the support. According to void fraction measurements (see Figure 4.15), the interface between the vapour pocket over the support and the liquid jet out of the broach hole leaves a low-void region directly over the support and near the broach-hole corner. Once a particle makes it to this region, it is protected from re-entrainment by the quasi-stagnant flow pattern over the support. If there is more flow mixing (as with lattice bars), the deposit is more likely to be swept away. 


\subsubsection{Particle Trapping}

It is not difficult to envisage that supports will act as "filters" by intercepting particles or agglomerates. Dynamic forces from the flow together with consolidation would keep the deposits in place (see Figure 4.5a). The chunky deposits found on the underside of the $1^{\text {st }}$ broached support plate in Pickering B (see Section 2.1.5) are a good example of this. The larger the flow area occupied by the support, the greater the deposit would be. If the deposit combines with that of the vena-contracta region (see previous section), this flow area occupied by the fouled support would become increasingly larger over time.

The deposition of particles downstream could be due to gravitational or centrifugal settling in a low-velocity zone above the downstream surface of the support. As mentioned in the previous section, these deposits are then protected from re-entrainment by the stable two-phase flow pattern in the wake region.

\subsubsection{Design Features}

The previous two sections discuss how design features such as sharp edges and large support surface areas could encourage deposition on the support. Another feature that may favor deposition is design symmetry leading to straight flow channels. The broached plate design, for example, leads to very strong flow jetting out of the broach hole which, in turn, gives very stable non-uniform velocity and void fraction distributions downstream of the support (see Figures 5.4a and 5.4c). Minimal mixing due to such static patterns would allow deposits to grow undisturbed in stagnant regions. In contrast, the staggered arrangement of lattice bars promote cross-flow and mixing (see Figure 5.4d), and hence reduced deposition. 


\subsubsection{Variation in Subchannel Size}

The flow visualization test with partially blocked channels in air/water (see Figure 4.4) indicated that the blocked (i.e., smaller) channels featured slow, liquid-rich fluid, while the more open (larger) channels featured fast, gas-rich fluid. This illustrated the concept of channels of different sizes having different flow conditions. Because parallel channels have the same overall pressure loss, the larger, less restrictive channel can afford to have greater two-phase pressure loss component and hence higher void and/or flow velocity than the smaller, more restrictive channel. What this means in terms of fouling is not clear-the smaller channel may foul preferentially due to its liquid-rich and hence particle-rich fluid, but this fluid is slower and the quantity of particles transported to that channel is therefore reduced. It can be said that a large variation in size will likely cause preferential fouling in one size of channel over the other. Again, predictions using TSFOUL were undertaken to clarify this behaviour, as described in Chapters 9 and 10.

\subsubsection{Effect of Location in SG}

Deposit thickness and morphology are to some extent a function of location in the SG. In general, two different mechanisms appear to be at play : (1) a hydraulic "filtering" mechanism near the bottom of the SG close to a source of large particles or agglomerates (brought in by the feedwater or from sludge piles on the tubesheet), and (2) a thermal "consolidation" mechanism near the top because of high quality and hence large pressure losses across the support.

In Pickering B, the bottom-most $\left(1^{s t}\right)$ broached plate was most fouled, suggesting that the filtering mechanism dominated. These units are reported to have thick layers of sludge on the tubesheet, i.e., a source of crud that is easily swept up to the underside of the $1^{\text {st }}$ plate. The deposits were reported to be chunky and crystalline, suggesting a filtering 
effect as large particles or agglomerates are more likely to impact on the underside of the support than small particles entrained in the flow.

In Bruce $A$, the last $\left(7^{\text {th }}\right)$ broached plate was most fouled, indicating a dominant thermal mechanism. These deposits consisted of only the very fine particles that could make it to the uppermost supports. Here, the pressure losses are highest (due to high quality and hence large two-phase multipliers), which would lead to the greatest extent of flashing and subsequently the most effective deposit consolidation.

\subsubsection{Effect of SG Design}

A further determinant for deposit distribution within the SG is believed to be the presence or absence of an intemal preheater, and its subsequent effect on flow distribution.

[Figures 1.3 and 1.4 show SGs with and without internal (or integral) preheater.] To show this, two-phase pressure losses across clean supports were calculated in a manner similar to that described in Section 7.3.5. for the Bruce A SG which has no internal preheater, and for the CANDU-6 SG, which has an internal preheater. Figures 6.2a and 6.2b show the approximate qualities and free-span mass fluxes on the hot leg of the two SGs. Note that the mass flux for the CANDU-6 is higher at the bottom because the lowflow preheater occupies the entire flow area on the cold-leg side. Figures 6.3a and 6.3b show the calculated pressure losses across the support-the overall loss is across the entire support, while the maximum loss is that across the inlet of the support (due to flow contraction and acceleration).

It appears that the greatest pressure loss in the axial profile occurs at an elevation that is related to whether the SG had an internal preheater or not. The peak loss occurs at the last (uppermost) support for an extemal-preheater SG, and at a middle support for the internal-preheater SG. In the former case, the peak is determined by the location of highest quality, while in the latter case, it is determined by a combination of moderate 
quality and high mass flux, because the flow area is halved next to the preheater. While this is by no means the only explanation for the fouling trend, it helps in the understanding of why units with internal preheater tend to have greater deposition in the lower SG regions, and vice-versa for the external-preheater SGs.

[These simple pressure loss calculations were undertaken before TSFOUL was created. At the time, they hinted at the usefulness of analytical models in predicting deposition, and served as starting points in the development of TSFOUL.]

\subsubsection{Effect of SG Conditions}

The dependence of deposition on steam quality is a complex one, as changes in quality are accompanied by changes in flow regime (e.g., bubbly flow versus churn flow) and in the manner of vapour generation (e.g., nucleate boiling versus film boiling) [1]. However, in general, an increase in quality is believed to favor deposition (due to the increased liquid velocity and hence increased transport of particles and solubles toward surfaces. Evidence for this is the tendency for the hot leg in Bruce A SGs to carry more deposits than the cold leg (see Section 2.1.3). In the OTSGs, in which the steam is superheated at the outlet, the deposition increases steadily with quality (but reaches a maximum at a certain quality ( $30-50 \%)$ after which the particle concentration is reduced and the particle-carrying liquid becomes progressively scarce).

\subsection{Criteria for Fouling Propensity}

From the flow characterization work (Chapters 4 and 5) and the correlations with field data (Sections 6.1.1 to 6.1.4), a list of criteria was drawn up to assess the fouling propensity of a given support design. This list pertains to the support itself, not to its location in the SG or the SG design and conditions. 
(1) Flow resistance: if the support presents a large obstruction to the flow (through sudden contractions, sharp edges, high friction loss, flow redirection, or sudden expansion), pressure losses would promote flashing, which may cause solubles to precipitate onto the support. The developed flow is then also disrupted, creating stagnant zones (e.g., at a vena contracta) where particles can collect. Rounded edges on especially the upstream side of the support help to reduce the flow resistance significantly.

(2) Particle trapping upstream: if the support has large land regions normal to the upstream flow, for example a land of comparable size to the flow channel, dynamic forces may trap particles and especially the larger agglomerates on the underside of the support.

(3) Particle deposition downstream: if the support has a sudden and large downstream flow expansion, because of a land of comparable size to the flow channel, flow recirculation or stagnation occurs (depending on the void fraction), which may cause particles to deposit on the topside of the support.

(4) Design symmetry: if the support is axially symmetric (i.e., flow channels are straight and unchanging in the axial direction), vapour pockets will form which may promote stagnant regions even under slug/churn conditions; in contrast, non-symmetric designs encourage flow mixing.

(5) Variation in subchannel size: if subchannels in the support vary greatly in size, the two channels will have dissimilar flow velocities and void fractions, i.e., different flow conditions, which will likely result in preferential deposition in one of the channels.

In Table 6.1, these criteria are used to qualitatively evaluate supports for their fouling propensity. For the Darlington lattice bars, a distinction is made between the high-bar and the low-bar regions. No attempt was made to weight the criteria according to relative importance, because this is still unclear. Also, factors such as vibration prevention. cost, 
and ease of assembly were not included in the assessment of the supports. This Table suggests that the relative fouling propensities are:

(WORST) broached plate $\ll$ lattice bars $<$ formed bars (BEST).

As deposition leading to flow instability has been reported in the flow holes of both trefoil and quatrefoil broached plate supports, and not with lattice bars or formed bars, the problem appears indeed to be generic to the broached design.

Figure 6.4 illustrates the proposed fouling mechanisms for supports. These are mechanisms gleaned from observations of deposits in the field and from flow visualization tests in the laboratory. The mechanisms will be examined further and refined using TSFOUL to predict deposition rates.

The next Chapter describes the analytical modeling of support deposition, starting with the basic equations for the flow conditions.

Reference

[1] D. Thomas, “An Investigation of Magnetite Deposition and its Influence on Heat Transfer in Steam Generator Tubes”, protected AECL report No. CRNL-1 132 , 1974 April, translated from German by K.A. Burrill from an unpublished internal report of Siemens Aktiengesellschaft, Erlangen, West Germany, 1969. 
Table 6.1: Qualitative evaluation of supports from fouling perspective

\begin{tabular}{|c|c|c|c|c|}
\hline $\begin{array}{l}\text { a fouling-resistant design will } \\
\text { have: }\end{array}$ & $\begin{array}{c}\text { Bruce } \\
\text { broached } \\
\text { plate }\end{array}$ & $\begin{array}{c}\text { CANDU-6 } \\
\text { broached } \\
\text { plate }\end{array}$ & $\begin{array}{l}\text { Darlington } \\
\text { lattice bars } \\
\text { high low }\end{array}$ & $\begin{array}{l}\text { Wolsong-1 } \\
\text { formed bars }\end{array}$ \\
\hline low flow resistance & $\mathbf{X}$ & $\mathbf{O}$ & $X \quad r$ & $\checkmark$ \\
\hline $\begin{array}{l}\text { low potential for particle trapping } \\
\text { upstream }\end{array}$ & $\mathbf{X}$ & $\mathbf{X}$ & $X \quad \checkmark$ & $\checkmark$ \\
\hline $\begin{array}{l}\text { low potential for particle } \\
\text { deposition downstream }\end{array}$ & $\mathrm{X}$ & $\mathbf{X}$ & $x \quad r$ & $\checkmark$ \\
\hline uniformity in subchannel size & $\checkmark$ & $\checkmark$ & O 0 & $\mathbf{O}$ \\
\hline design asymmetry for flow mixing & $\mathbf{X}$ & $\mathbf{X}$ & $\checkmark \checkmark$ & $\mathbf{X}$ \\
\hline NET ASSESSMENT & $\mathbf{X X X}$ & $\mathbf{X X}$ & $\checkmark$ & $\checkmark \checkmark$ \\
\hline
\end{tabular}

legend: $\quad X=b a d$

$$
\begin{aligned}
& \mathrm{O}=\mathbf{o . k} . \\
& \checkmark=\text { good }
\end{aligned}
$$

\section{NOTES:}

- For the Darlington lattice bars, a distinction is made between the high-bar and the low-bar regions.

- No attempt was made to weight the criteria according to relative importance. Also, factors such as vibration prevention, cost, and ease of assembly were not included in the assessment. 
Figure 6.1: Schematic of vena contracta region

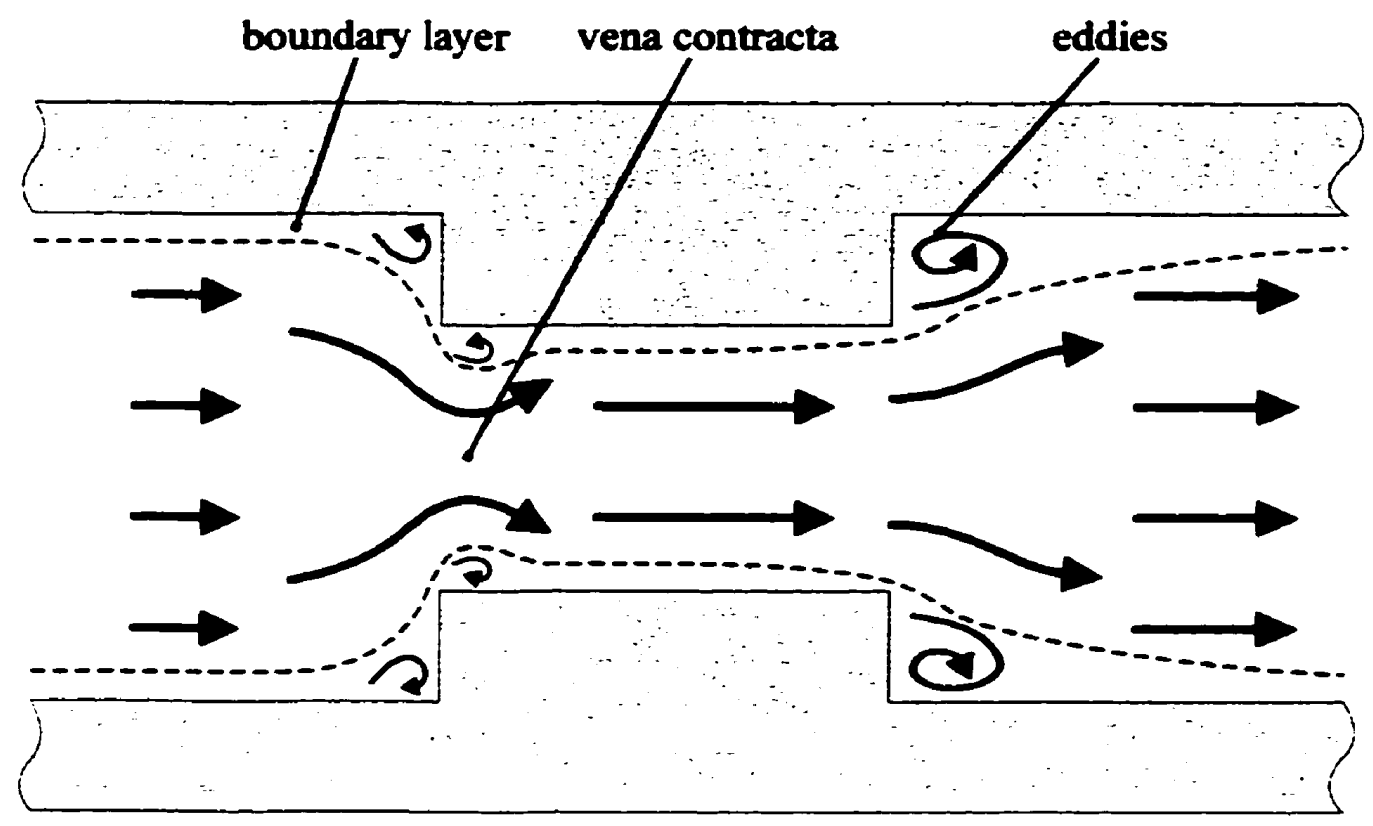


Figure 6.2: Approximate conditions on SG hot leg

(a) quality

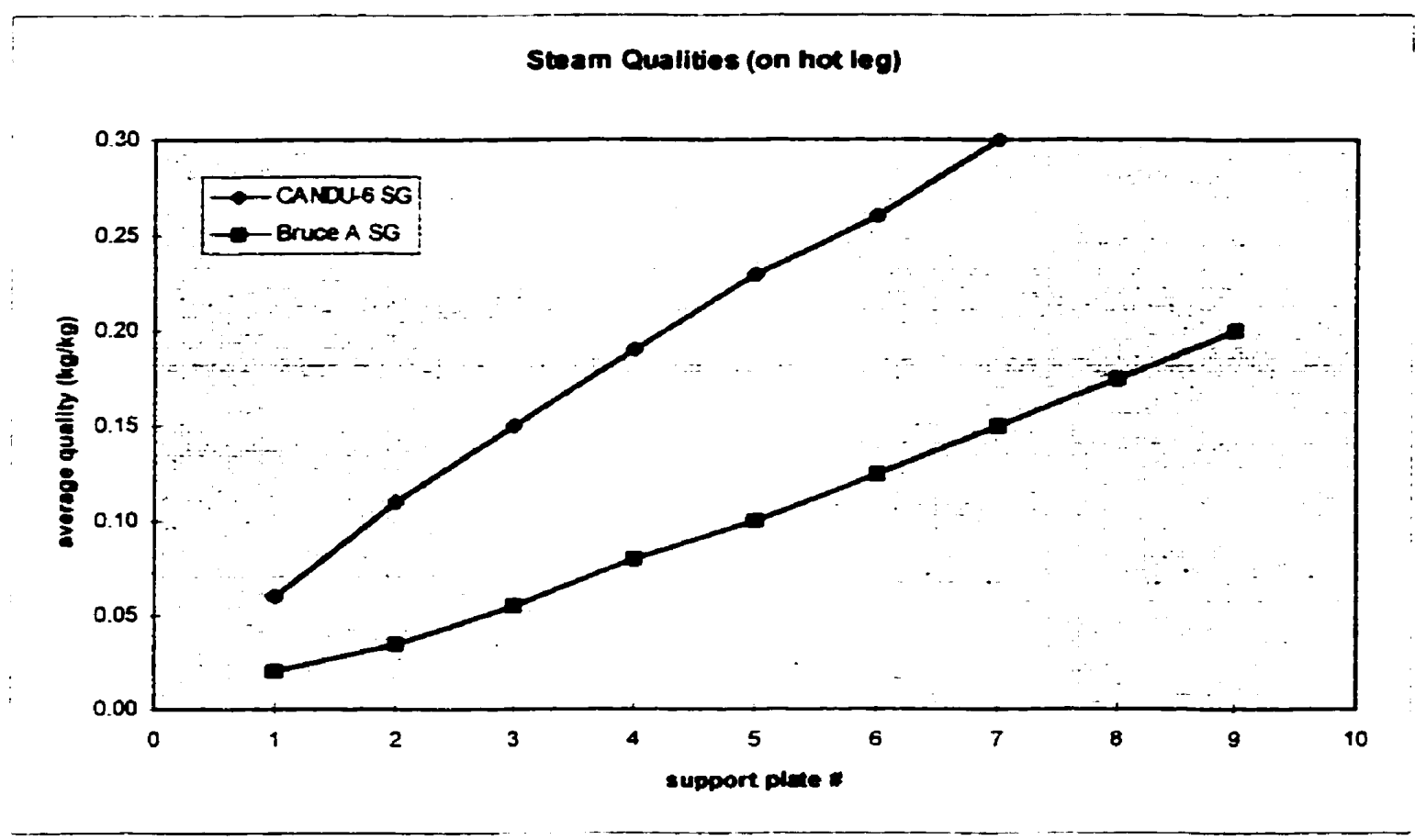

(b) mass flux

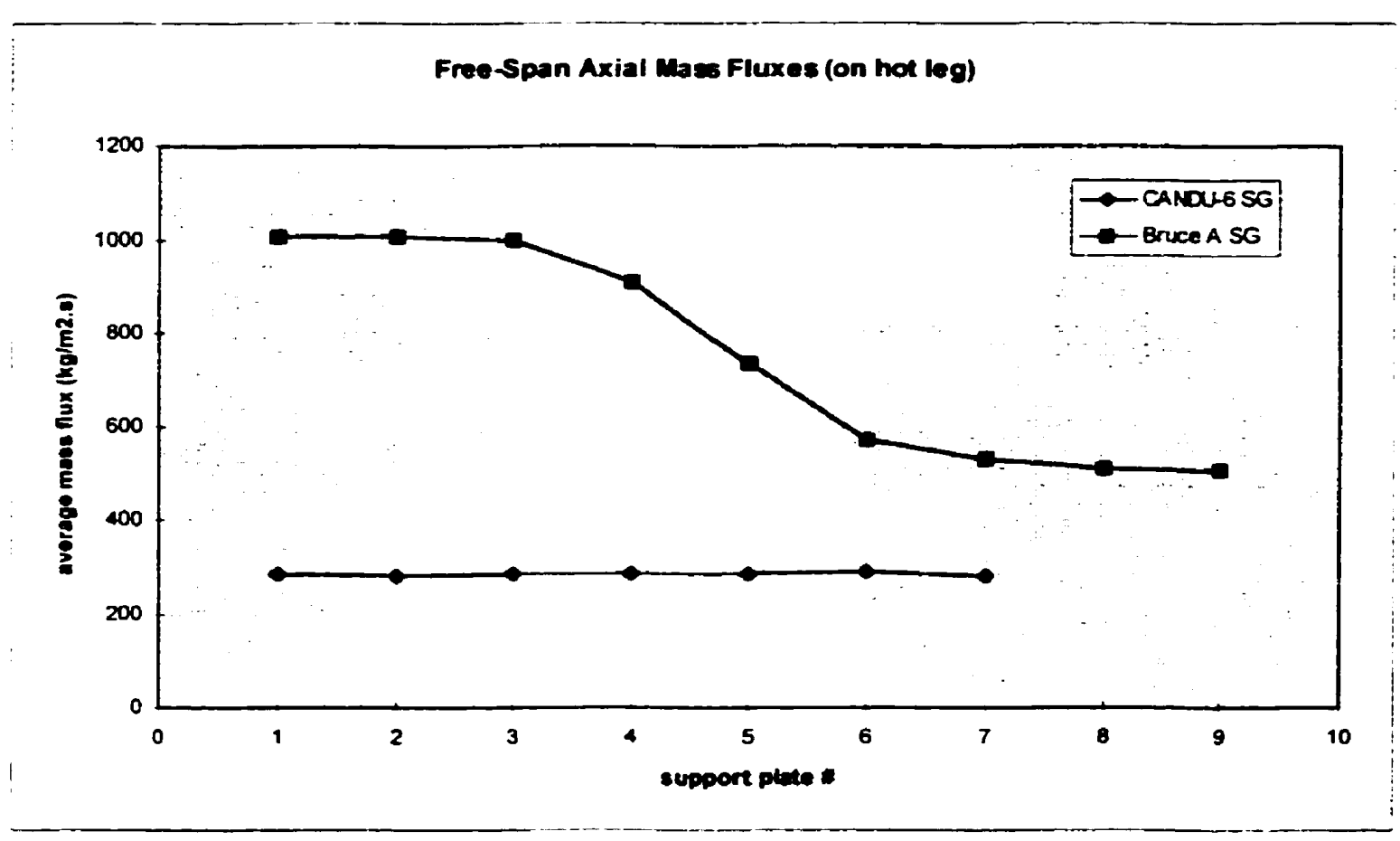


Figure 6.3: Calculated tube-support pressure losses:

(a) overall loss across support

Two-Phas OVERALL DPs acros Support (on hot leg)

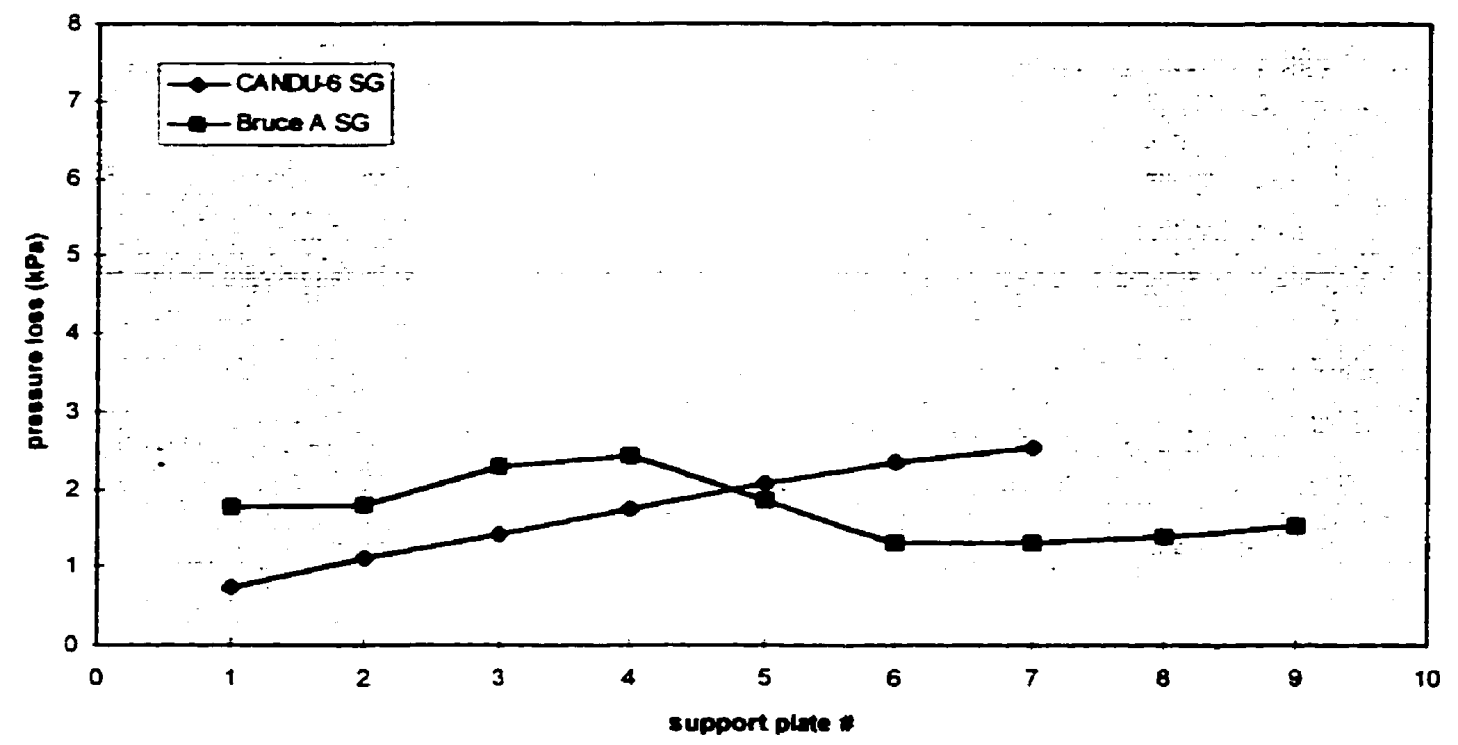

(b) maximum loss (at support inlet)

Two-Phas MAxImum DPs across Support (on hot log)

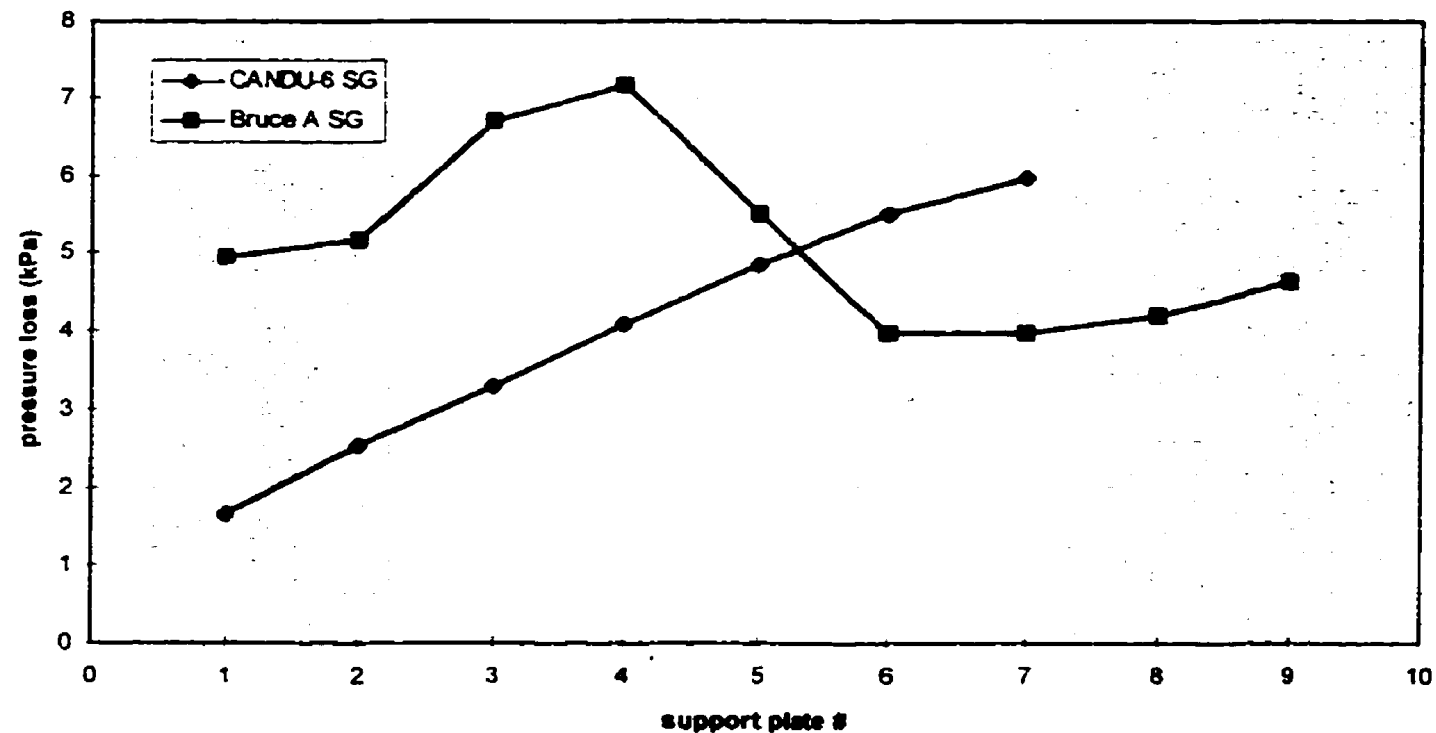


Figure 6.4: Proposed support fouling mechanisms

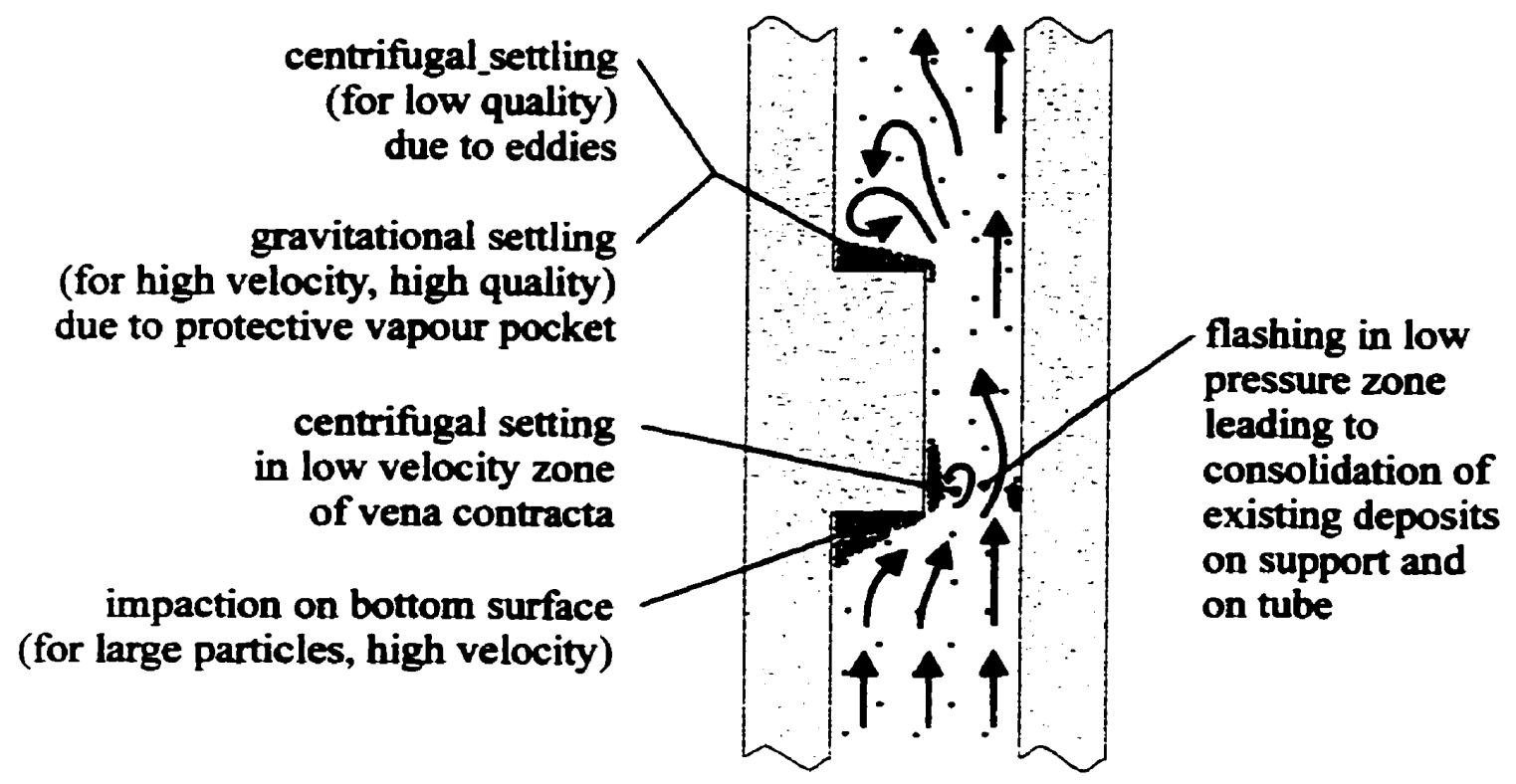




\section{Chapter 7}

\section{TSFOUL: BASIC ANAL YTICAL MODELS}

\subsection{General Description of TSFOUL}

A simple computer program called TSFOUL (Tube-Support FOULing) was developed to predict the rate of deposition on supports analytically. The main objective of TSFOUL was to help understand and establish the reasons for blockage of the supports in the SGs. Eventually, such a tool could also provide the "fouling susceptibility" of particular supports when they are modeled in the larger codes.

The basic equations for flow conditions in TSFOUL are described in this Chapter. Chapters 8 and 9 describes the deposition models in TSFOUL.

\subsection{Basic Program Structure}

As shown in Figure 7.1, the geometry in TSFOUL is that of a one-dimensional annular flow channel. The inner surface of the annulus represents the heated SG tube. The outer surface gives the proper flow channel width, especially in the support region where the channel narrows. The channel widths are determined by starting with the actual crosssectional flow area of the free-span region or the support region in the SG, and finding the width that will give this flow area in an annular configuration. TSFOUL is currently set up for a broached plate geometry, i.e., only one narrowing.

The fluid is a two-phase steam/water mixture, with particles flowing with the liquid phase. The specified conditions at the inlet are: thermodynamic quality, pressure, fluid temperature, surface temperatures, tube heat flux, initial mass flux, particle concentration, particle diameter, particle properties, and channel dimensions. The main output is the 
deposit thickness after a given time, at a given axial location, for heated tube surface and unheated support surface.

The inlet conditions are assumed to remain constant over time, except for the mass flux which is made to decrease as a function of the increase in the support flow resistance with time (as presented in Section 7.3.7). In the thermosyphoning system of the CANDU SGs. fouling of the supports reduces the flow clearance and increases flow resistance; the flow rate must drop because of the constant driving head. This then affects a host of flowdependent parameters.

As shown in Figure 7.1, the flow path is discretized using 65 nodes, each $1 \mathrm{~mm}$ in height:

- Nodes 1 to 20 represent the upstream free-span region (tubes only, no support).

- Nodes 21 to 45 represent the support region. TSFOUL allows for splitting of the flow between two (unequally-sized) independent annular channels $\mathrm{Y}$ and $\mathrm{Z}$.

- Nodes 46 to 65 represent the downstream free-span region.

The calculation sequence is shown in the flowchart of Figure 7.2. In essence, at a given time $t$ and for a given node $i$. (1) flow conditions are established, (2) deposition coefficients are calculated, and finally (3) the change in deposit thickness is determined. In the support region, Step 1 requires a lengthy iteration to establish the flow split, based on equalizing the two-phase pressure loss across the $\mathrm{Y}$ and $\mathrm{Z}$ channels.

\subsection{Basic Thermalhydraulic Models}

\subsubsection{Fluid Physical Properties}

TSFOUL calculates the physical properties of the steam/water mixture as a function of pressure and temperature. The functions used to calculate the physical properties of light water are low-order polynomial fits to the 1984 NBS/NRC Steam Tables, and are fast- 
running and reasonably accurate ( $0.1 \%$ error) $[1]$. Only the temperature and pressure ranges of interest to steam generators were used. The calculated properties are: $T_{\text {sat }}, P_{\text {sal }}$, specific heat $C p$, density $\rho$, enthalpy $h$, viscosities $\mu$ and $v$, and thermal conductivity $k$.

\subsubsection{Mass Quality}

A simple method is described in Collier [2] to obtain mass quality using correlations for the bulk temperature at which the onset of significant void (OSV) occurs. [It is assumed that essentially no vapour is produced below that temperature.] It is based on work by Saha \& Zuber [3] who proposed calculating the subcooling $\Delta \mathbf{T}_{\text {sub }}$ at the point of "net vapour generation", which is assumed to coincide with the point of bubble detachment and hence OSV. This detachment is thermally-controlled at low flow rates (i.e., low values of $\mathrm{Pe}=\operatorname{Re} \mathrm{Pr}$ ) and hydrodynamically-induced at high flow rates (high $\mathrm{Pe}$ ):

$$
\begin{aligned}
& \text { for } P e<70000 \quad \Delta T_{\text {sub.bd }}=0.0022 \frac{Q D_{h y}}{k_{f}} \\
& \text { for } P e>70000 \quad \Delta T_{\text {sut }, b d}=153.8 \frac{Q}{G C p_{f}}
\end{aligned}
$$

If $T_{b}>\left(T_{s a r}-\Delta T_{\text {sub.bd }}\right)$ it is assumed that there is net vapour generation. The empirical (curve fit) method by Levy [4] is then used to obtain the mass quality $\chi$ from the thermodynamic quality $\chi_{\text {th }}$ :

$$
\chi=\chi_{c h}-\chi_{b d} \exp \left(\frac{\chi_{t h}}{\chi_{b d}}-1\right)
$$

where $\chi_{\text {bd }}$ is the (negative) $\chi_{\mathrm{Lh}}$ at the point of bubble detachment : 


$$
\chi_{b d}=-\frac{C p_{f} \Delta T_{\text {subpd }}}{h_{f g}}
$$

Note that if $\chi_{b d}=0$, then the mass quality $\chi$ equals $\chi_{\text {th. }}$. Should $\chi_{\text {bd }} \geq \chi_{\text {th }}$, an error occurs and the mass quality $\chi$ is set to zero.

\subsubsection{Void Fraction}

The void fraction $\alpha$ is calculated from the superficial liquid velocity $\mathrm{j}_{\mathrm{f}}$ and mass quality $\chi$ using the drift flux model [2]:

$$
\alpha=\frac{j_{g}}{C_{o}\left(j_{f}+j_{g}\right)+V_{g}}
$$

where the superficial gas and liquid velocities are defined as:

$$
j_{g}=\frac{G \chi}{\rho_{g}} \quad j_{f}=\frac{G(1-\chi)}{\rho_{f}}
$$

Following work done by Unal [5] for the bubble/plug flow regimes during boiling at elevated pressures, the void distribution parameter $C_{0}$ is set to 1.03 , and the weighted mean drift velocity $V_{g j}$ for this regime in an annular geometry is expressed as:

$$
V_{g}=40.25\left[\frac{\mu_{f} g\left(\rho_{f}-\rho_{g}\right)}{\rho_{f}^{2}}\right]^{1 / 3}
$$




\subsubsection{Friction Factor}

The Darcy friction factor $f$ is used to compute the pressure loss due to friction $\Delta P_{\text {fric }}$ and the friction velocity $u^{*}$. For two-phase friction pressure loss (described in the next section) and following conventional procedure, $f$ is based on the all-liquid Reynolds number, i.e., it is assumed to be equal to that which would have occurred had the total flow been all liquid [2]:

$$
\operatorname{Re}_{f}=\frac{G D_{h y}}{\mu_{f}}
$$

For the friction velocity $u^{*}$ (defined in Equation A.5 in Appendix A), $f$ is based on a separated-flow Reynolds number, which considers only the liquid portion of the twophase flow:

$$
\operatorname{Re}_{f u}=\frac{G(1-\chi) D_{h y}}{(1-\alpha) \mu_{f}}=\operatorname{Re}_{f}\left(\frac{1-\chi}{1-\alpha}\right)
$$

The $(1-\chi)$ term converts the total mass flux to the liquid mass flux, and the $(1-\alpha)$ term accounts for an effectively smaller channel in which the velocity is higher. Note that the hydraulic diameter remains that of the actual channel, making this $\mathbf{R e}_{\mathrm{fu}}$ an approximate parameter only.)

For laminar flow $(\operatorname{Re}<\approx 3000)[6]$,

$$
f_{L}=\frac{64}{\mathbf{R e}}
$$

For turbulent flow $(\operatorname{Re}>\approx 3000$ ), for walls characterized by a roughness $\varepsilon[6,7]$, 


$$
f_{\tau}=\left\{-1.8 \log _{10}\left[\frac{\operatorname{Re}_{f}}{6.9}+\left(\frac{\varepsilon / D_{h y}}{3.7}\right)^{1.11}\right]\right\}^{-2}
$$

To avoid calculation problems due to the discontinuity between the laminar and turbulent friction factors, a smoothing function is applied (which conveniently eliminates the need to specify a transition $\operatorname{Re}$ value such as “3000”) :

$$
f=\left(f_{L}^{3}+f_{T}^{3}\right)^{1 / 3}
$$

For friction pressure loss, $\operatorname{Re}_{\mathrm{f}}$ is used in Equations 7.9 to 7.11 ; for the friction velocity $\mathbf{u}^{*}$, $\mathrm{Re}_{\mathrm{fu}}$ is used instead.

\subsubsection{Two-Phase Pressure Loss}

The flow rates in the two support channels are determined iteratively by seeking equal pressure loss across each channel. For this, a two-phase pressure-loss model was needed. In TSFOUL, this model is also used to calculate an axial pressure profile (i.e., the pressure at each node), as well as the overall support flow resistance for the purpose of updating the mass flux at each time step (as shown in Section 7.3.7). For pressure loss calculations, the fluid is assumed to be homogeneous. ${ }^{7}$

A comparison with Idel'chik's model [8] of overall single-phase pressure loss across a thick-edged orifice is described in Appendix B. This comparison showed reasonable agreement between the two models, giving confidence that the model described here is appropriate and was implemented properly in TSFOUL.

\footnotetext{
'This was done for practical reasons-the homogeneous model is easier to implement than the drift flux model used for void fraction (Section 7.3.3), and the difference is expected to be slight.
} 


\section{$\underline{\text { Overall } \Delta P}$}

The total pressure difference $\Delta P_{\text {total }}$ between one node " $\mathrm{i}$ " and the previous node " $\mathrm{i}-1$ " is a sum of pressure changes due to gravitational (static) head, acceleration arising from quality increase, friction, sudden contraction, sudden expansion, actual area changes, effective area changes (from vena contracta effects), and flow development:

$$
\Delta P_{\text {tol }}=\Delta P_{g^{r}}+\Delta P_{\text {axc }}+\Delta P_{\text {frc }}+\Delta P_{\text {con } / \text { exp }}+\Delta P_{\text {areal } / \text { ena }}+\Delta P_{\text {fddev }}
$$

In TSFOUL, the pressure difference is taken to be positive if the pressure decreases in the direction of the flow. The correlations for the two-phase multiplier terms are taken from Collier [2], for homogeneous flow.

\section{Static head $\Delta P$}

This term describes the change in pressure due to a change in elevation, and hence static head in the steam generator. For a given node $i$,

$$
\Delta P_{g r}=\rho_{1} g \Delta z
$$

The average density $\rho$ is obtained assuming a homogeneous mixture [2]:

$$
\rho=\alpha \rho_{g}+(1-\alpha) \rho_{f}
$$

\section{Acceleration $\Delta P$}

This term describes the change in pressure due to fluid acceleration or deceleration caused by changes in mass quality (due to boiling). For a given node i, 


$$
\Delta P_{\text {acc }}=G^{2}\left(\frac{1}{\rho_{g}}-\frac{1}{\rho_{f}}\right)\left(\chi_{t}-\chi_{t-1}\right)
$$

\section{Friction $\Delta \mathrm{P}$}

This term describes the non-recoverable change in pressure due to friction along surfaces. For a given node $i$,

$$
\Delta P_{f n c}=\frac{G^{2}}{2 \rho_{f}} K_{f n c} \Phi_{f n c}^{2}
$$

$\mathrm{K}_{\text {fric }}$ is the frictional flow-resistance coefficient defined by :

$$
K_{f r c c}=f \frac{\Delta z}{D_{h y}}
$$

where $f$ is defined in Equations 7.9 to 7.11 . The two-phase multiplier $\Phi_{\text {fric }}^{2}$ is regimedependent [2]:

$$
\begin{aligned}
& \text { laminar } \quad \Phi_{f n c, L}^{2}=1+\chi\left(\frac{\rho_{f}}{\rho_{g}} \frac{\mu_{g}}{\mu_{f}}-1\right) \\
& \text { turbulent } \quad \Phi_{f n c . T}^{2}=\left[1+\chi\left(\frac{\rho_{f}}{\rho_{g}}-1\right)\right]\left[1+\chi\left(\frac{\mu_{f}}{\mu_{g}}-1\right)\right]^{-0.25}
\end{aligned}
$$

To ensure a smooth transition between the two conditions, a smoothing function is applied as in Equation 7.11. Because this two-phase multiplier is only used with the friction factor, the friction factor itself is carried in the smoothing process, to avoid having to do each term separately: 


$$
f \Phi_{f n c}^{2}=\left(\left(f_{L} \Phi_{f n c, L}^{2}\right)^{3}+\left(f_{T} \Phi_{f n c, T}^{2}\right)^{3}\right)^{1 / 3}
$$

\section{Contraction $\Delta \mathbf{P}$}

This term describes the non-recoverable change in pressure due to sudden flow contraction at the inlet of the support. At the first node inside the support, i.e., after the area reduction,

$$
\begin{aligned}
& \Delta P_{c o n}=\frac{G_{t}^{2}}{2 \rho_{f}} K_{c o n} \Phi_{c o n}^{2} \\
& \text { where } G_{t}=G_{t-1} \frac{A_{t-1}}{A_{t}} \\
& K_{\text {con }}=\left(\frac{1}{C_{c}}-1\right)^{2} \\
& \Phi_{c o n}^{2}=1+\left(\frac{\rho_{f}}{\rho_{g}}-1\right) \chi
\end{aligned}
$$

$C_{c}$ is the ratio of the vena contracta area to $A_{i}$ (i.e., the fraction of effective flow area) and is a function of $\mathbf{A}_{\mathbf{i}} / \mathbf{A}_{\mathrm{i}-\mathrm{l}}$, as tabulated in Collier [2]. The quality is assumed to remain constant as the flow contracts; while this is not really true, the model represents experimental data well [2]. The pressure loss due to contraction is assumed to be a onetime loss that occurs at the first node inside the support. 


\section{Expansion $\Delta \mathrm{P}$}

This term describes the non-recoverable change in pressure due to sudden flow expansion past the outlet of the support. At the first node outside the support, i.e., after the area increase,

$$
\begin{aligned}
& \Delta P_{\exp }=\frac{G_{t-1}^{2}}{2 \rho_{f}} K_{\exp } \Phi_{\exp }^{2} \\
& \text { where } \quad G_{t-1}=G_{t} \frac{A_{t}}{A_{t-1}} \\
& K_{\exp }=\left(1-\frac{A_{t-1}}{A_{t}}\right)^{2} \\
& \Phi_{\exp }^{2}=1+\left(\frac{\rho_{f}}{\rho_{g}}-1\right) \chi
\end{aligned}
$$

The pressure loss due to expansion is assumed to be a one-time loss that occurs immediately downstream of the support. [In practice, the expansion would be gradual owing to jetting of the fluid out of the support channel.]

\section{Area change $\Delta P$}

This term describes the change in pressure due to area changes. Unlike other pressure changes, these losses are recoverable-no net loss if the downstream density and quality are the same as the upstream values, and if a contraction is followed by an expansion to the original flow area. The pressure change occurs from the Bemoulli effect: pressure is lost as the fluid velocity increases in a reduced flow area (and vice-versa). 
There are three area changes modeled in TSFOUL. The first is the area reduction (i.e., a pressure loss) from the free span to the vena contracta at the support inlet. Note that this is an effective area reduction that is greater than the actual physical area change, owing to the vena contracta effect. At the first node inside the support,

$$
\begin{aligned}
& \Delta P_{\text {vena }}=\frac{G_{\text {vena }}^{2}}{2 \rho_{f}} K_{\text {vena }} \Phi_{\text {vena }}^{2} \\
& \text { where } \quad G_{\text {vena }}=G_{i-1} \frac{A_{t-1}}{A_{\text {vena.s }}} \\
& K_{\text {vena }}=1-\left(\frac{A_{\text {vena },}}{A_{t-1}}\right)^{2} \\
& \Phi_{\text {vena }}^{2}=1+\left(\frac{\rho_{f}}{\rho_{g}}-1\right) \chi \\
& A_{\text {venas }}=C_{c} A_{t}
\end{aligned}
$$

The second area change is an area increase (i.e., a pressure gain) between the vena contracta region and the actual support channel, as the boundary layer re-attaches and the flow re-develops. At the first node after the boundary layer re-attachment (i.e., part-way into the support),

$$
\begin{aligned}
& \Delta P_{\text {vena }}=\frac{G_{i}^{2}}{2 \rho_{f}} K_{\text {vena }} \Phi_{\text {vena }}^{2} \\
& K_{\text {vena }}=1-\left(\frac{A_{t}}{A_{\text {vena },-1}}\right)^{2}
\end{aligned}
$$


$\Phi^{2}$ vena is the same as in Equation 7.32, except that it uses local densities and mass quality.

The end of the vena contracta region is assumed to be at $\mathrm{L} / \mathrm{D}_{\text {hy }} \approx 2$ (starting from the support inlet) in single phase. This is based on pressure-loss measurements in singlephase Freon-11 on the broached plate mockup (which had $D_{\text {hy }}=6 \mathrm{~mm}$ ), in which pressure recovery associated with boundary layer re-attachment beyond the inlet occurred after 10 to $15 \mathrm{~mm}$ (see Figure 5.6, top). Measurements in two-phase Freon-11 on the same geometry indicate that pressure recovery is delayed to about $L / D_{\text {hy }} \approx 4$ (see Figure 5.6. bottom). Thus, the end of the vena contracta region is set at $L / D_{\text {hy }}=2$ if the mass quality $\chi \leq 0$, and set at $L / D_{\text {hy }}=4$ if $\chi>0$.

The third area change is an area increase (i.e.. a pressure gain) between the support channel at the outlet and the downstream free-span region. At the first node outside the support,

$$
\begin{aligned}
& \Delta P_{\text {area }}=\frac{G_{t-1}^{2}}{2 \rho_{f}} K_{\text {area }} \Phi_{\text {area }}^{2} \\
& \text { where } \quad K_{\text {area }}=1-\left(\frac{A_{t}}{A_{t-l}}\right)^{2} \\
& \Phi_{\text {area }}^{2}=1+\left(\frac{\rho_{f}}{\rho_{g}}-1\right) \chi
\end{aligned}
$$

\section{Flow development $\Delta P$}

This term describes the change in pressure due to flow development at the inlet of the support. The loss is caused by the acceleration of flow from a uniform profile to a fullydeveloped one, and caused by increased shear in the entrance boundary layers. For a given node $\mathrm{i}$, 


$$
\begin{aligned}
& \Delta P_{\text {fdev }}=\frac{G_{t}^{2}}{2 \rho_{f}} K_{f d d v} \Phi_{f d d e v}^{2} \\
& K_{\text {fdev }}=\tau_{t}\left(1-\frac{A_{t}}{A_{f s}}\right)^{1.375} \\
& \Phi_{\text {fdev }}^{2}=1+\left(\frac{\rho_{f}}{\rho_{g}}-1\right) \chi
\end{aligned}
$$

This expression for $\mathbf{K}_{\text {fldev }}$ is based on Idel'chik's model for a thick-edged orifice in a straight channel under turbulent conditions [8], as described in Appendix B. It includes a flow development parameter $\tau$ which is a function of distance $z / D_{\text {hy }}$, where $z$ is the distance from the support inlet. In Idel'chik's model, $\tau$ is an average value used in calculating overall pressure loss; TSFOUL requires a local value $\tau_{i}$ to calculate pressure loss across individual nodes. Section 8.3.1 describes a method of obtaining local values from average ones, which can be adapted here:

$$
\tau_{1}=\tau_{\text {ove } z}+\frac{d \tau_{\text {ave } z}}{d z} z
$$

The first term, $\tau_{\text {ave, }, z}$, was obtained by curve-fitting a polynomial to Idel'chik's $\tau$ values [8]. The second term was obtained by differentiating this polynomial. Thus, for $\mathrm{X}=\mathbf{z} / \mathrm{D}_{\mathrm{hy}}$,

$$
\begin{aligned}
& \tau_{\text {ave } z}=-0.557 X^{4}+2.61 X^{3}-3.49 X^{2}+0.354 X+1.33 \\
& \frac{d \tau_{\text {ave, } z}}{d z} z=-2.23 X^{4}+7.84 X^{3}-6.99 X^{2}+0.354 X
\end{aligned}
$$


This model predicts that, in turbulent flow, the local value $\tau_{i}$ will drop to zero between $\mathrm{X}=0$ and $\mathrm{X} \approx 0.56$, i.e., over only a few $1-\mathrm{mm}$ nodes. In laminar flow, the flow development length is expected to be much longer, but this has little impact on pressure as the pressure losses are already very small.

\subsubsection{Variation of Quality over Distance}

Boiling on the tube surface changes the quality as the fluid travels along. The change in quality can be obtained from a heat balance: the heat generated on the tube surface equals the change in heat content of the fluid. Under thermally-saturated conditions,

$$
Q \pi D_{\text {tube }} \Delta z=m h_{f \mathcal{E}} \Delta \chi
$$

or $\quad \chi_{t}=\chi_{t-1}+\frac{Q D_{t u b e} \Delta z}{G\left(D_{\text {tube }}+w\right) w h_{f y}}$

Note that flashing is ignored in the variation of quality; in practice, flashing is not expected to contribute as much vapour to the fluid as boiling from the heated surface.

\subsubsection{Variation of Mass Flux over Time}

As discussed in Chapter 1 , the SG is a thermosyphoning system in which the pressure losses are approximately constant with time, since the buoyant force pressure difference which drives the llow is constant with time. [Under base-load conditions, the system parameters affecting the pressure difference, i.e., the total heat transfer rate, total steam and feedwater flow rates, the inlet temperature and the outlet steam quality, remain relatively constant over time.] The initial flow rate or mass flux must therefore decrease as the flow resistance increases with time due to growth of a deposit that encroaches on 
the flow channel. The flow rate reduction then continues until two-phase instability occurs [9]. Note that there will probably be some redistribution of pressure losses with time over the various components of the flow path (e.g., the downcomer annulus versus the supports), but this is a second-order effect.

In TSFOUL, the mass flux at the inlet (Node 1) is obtained by iteration after the first time step, to make sure it remains compatible with a fixed overall pressure loss. The steps are as follows:

1. For the first time $\operatorname{step}(\mathrm{J}=1)$, the inlet $\mathrm{G}$ is the input value. Calculate all pressure losses and deposit thicknesses based on this $G$ value. The overall pressure loss $\left(P_{i=1}-P_{i=65}\right)$ is called $\Delta P_{f i x e d}$; it must remain constant over time.

2. If there is no more time step, stop. Otherwise, go to the next time step.

3. Use this $G$ value to calculate all pressure losses and deposit thicknesses in this new time step. Calculate the overall loss $\Delta P_{\text {overall }}$ as $\left(P_{i=1}-P_{i=65}\right)$

4. Calculate the difference between $\Delta P_{\text {overall }}$ from Step 3 and $\Delta P_{\text {fixed }}$ from Step 1 . If the difference is less than $1 \%$ of the overall loss, then the $G$ value is appropriate; go to Step 2. If not, continue.

5. Use this difference and a derivative to adjust the $G$, now called $G_{\text {prev }}$ (meaning previous mass flux):

$$
\begin{aligned}
& G_{\text {new }}=G_{\text {prev }}+\frac{\Delta P_{\text {fized }}-\Delta P_{\text {overall }}}{d \Delta P / d G} \\
& \text { where } \frac{d \Delta P}{d G}=\frac{G}{\rho} K=\frac{2 \Delta P_{\text {overall }}}{G_{\text {prev }}}
\end{aligned}
$$

This equation assumes that $\mathrm{K}$ is constant with respect to $\mathrm{G}$; to a first approximation it is. $\Delta \mathbf{P}_{\text {overall }}$ is calculated by summing the $\Delta \mathbf{P}_{\text {to }}$ values (see Equation 7.12) for each node, from the first node to the last node. 
6. Go to Step 3 with $G_{\text {new }}$.

The derivative is helpful in ensuring quick convergence - typically, convergence is achieved in 3 or 4 iterations. This is important as the entire TSFOUL calculation of deposition in each node- which includes a sub-iteration for channel mass fluxes when the $\mathrm{Y}$ and $\mathrm{Z}$ channels are unequally sized-needs to be repeated in each iteration.

The next Chapter describes the deposition models in TSFOUL.

\section{References}

[1] W.J. Garland and J.D. Hoskins, “Approximate Functions for the Fast Calculation of Light-Water Properties at Saturation", International Journal of Multiphase Flow, Vol. 14, No. 3, pp. 333-348 (1988).

[2] J.G. Collier, Convective Boiling and Condensation, 2nd edition, McGraw-Hill Book Company, 1981.

[3] P. Saha and N. Zuber, "Point of Net Vapour Generation and Vapour Void Fraction in Subcooled Boiling", Proceedings of the 5th International Heat Transfer Conference, Tokyo, Paper B4.7 (1974).

[4] S. Levy, "Forced Convection Subcooled Boiling Prediction of Vapour Volumetric Fraction", International Journal of Heat \& Mass Transfer, Vol.10, pp.951-965 (1967). 
[5] H.C. Unal, "Determination of the Drift Velocity and the Void Fraction for the Bubble- and Plug-Flow Regimes during the Flow Boiling of Water at Elevated Pressures”, International Journal of Heat \& Mass Transfer, Vol.21, pp.1049-1056 (1978).

[6] F.M. White, Fluid Mechanics (2 ${ }^{\text {nd }}$ edition), McGraw-Hill Book Company, New York (1986).

[7] S.E. Haaland, "Simple and Explicit Formulas for the Friction Factor in Turbulent Pipe Flow", Journal of Fluids Engineering, 1983 March, pp.89-90.

[8] I.E. Idel'chik, Handbook of Hydraulic Resistance, $3^{\text {rd }}$ edition, CRC Press Inc., 1994, p. 222.

[9] M. Ishii and N. Zuber, "Thermally Induced Flow Instabilities in Two-Phase Mixtures", Paper B5.11, Proceedings of $4^{\text {th }}$ International Heat Transfer Conference, Paris, 1970. 
Figure 7.1: Geometry for TSFOUL

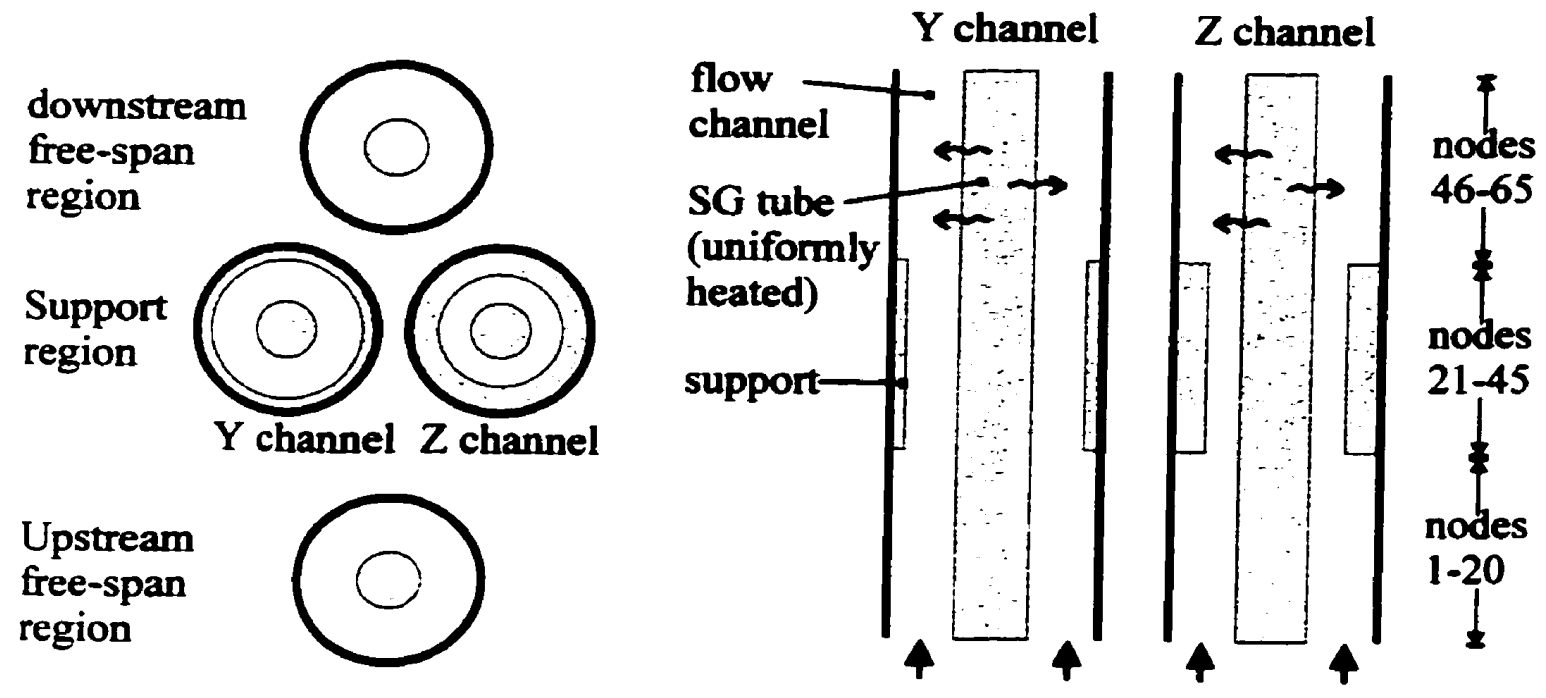


Figure 7.2: Flowchart for TSFOUL

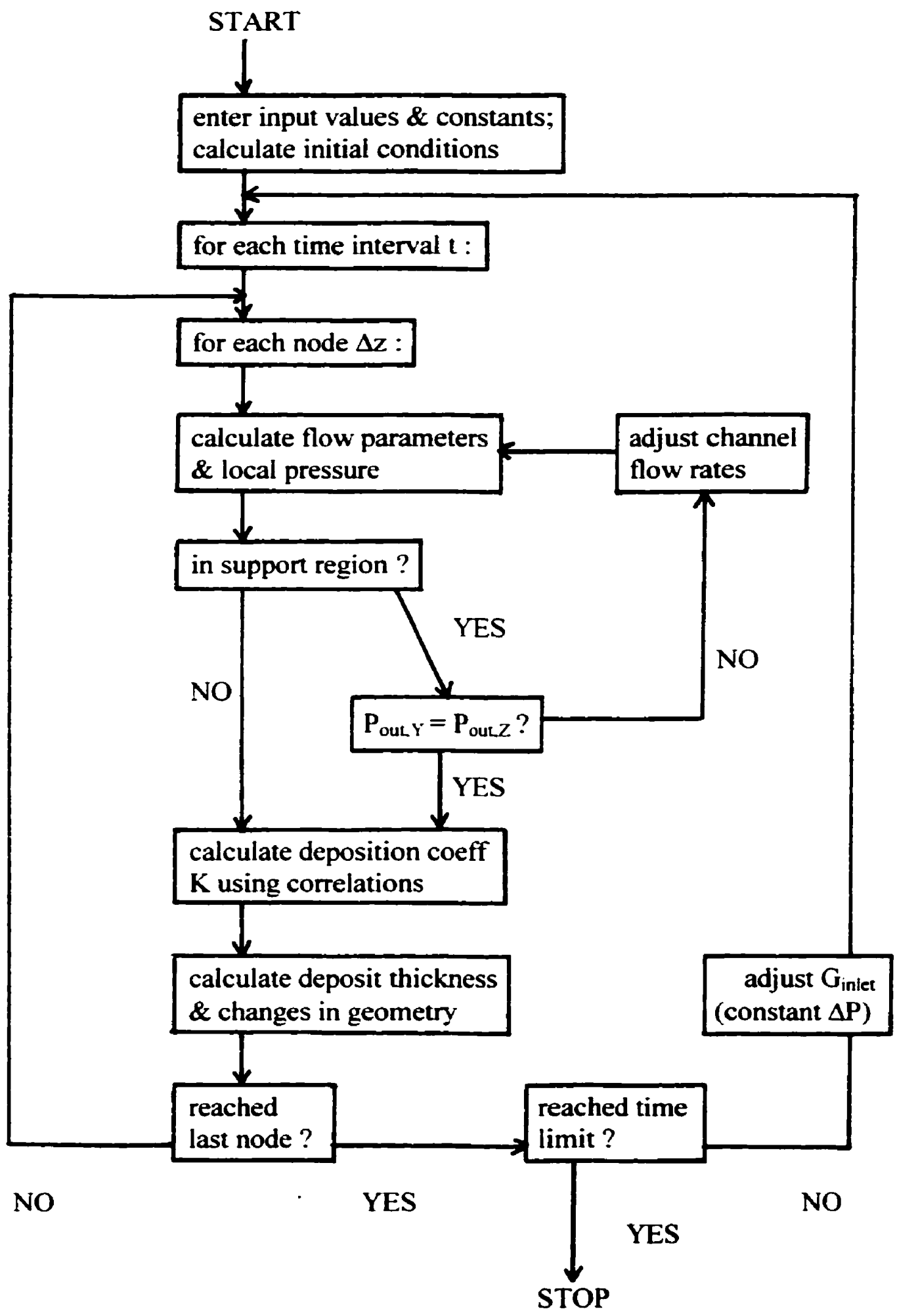




\section{Chapter 8}

\section{TSFOUL: BASIC DEPOSITION MODELS}

The basic equations governing particle behaviour used in the SLUDGE code [1] are described in Appendix A. This Chapter describes the refinements made to those equations for use in the TSFOUL program, and follows the same format as in Appendix A to facilitate comparison between SLUDGE and TSFOUL. Some discussion is included here to explore the origin of the deposition coefficients and other parameters.

\subsection{Governing Equations}

\subsubsection{Deposition in Turbulent Flow}

The deposit thickness $\delta$ after time $t$ for turbulent flow is as outlined in Equations A.6 and A.7 (in Appendix A):

$$
\delta=\frac{K \phi \rho}{\rho_{s}\left(1-\beta_{s}\right)} \frac{v_{f}}{A\left(u^{*}\right)^{2}}\left[1-\exp \left(\frac{-A t\left(u^{*}\right)^{2}}{v_{f}}\right)\right]
$$

The intent of the exponential expression is to account for the deposition rate in turbulent flow reaching an asymptote with time. This plateau effect has been attributed in the past to "turbulent down-sweeps" that remove deposited particles [2]. Epstein [3] argued that this leads to a "conceptual problem" of how particles ever get deposited if they are subjected to a re-entrainment mechanism. Various authors $[4,5]$ suggest a more plausible explanation based on the particle lift force analyzed by Saffman [6], which is the force experienced by a rotating object in a shear flow (-the so-called Magnus effect). They claim that it is hydrodynamic lift forces that encourage or discourage particles from 
depositing in the first place. According to Rouhiainen and Stachiewicz [5], in laminar flow, the lift force is negligible for small particles $\left(D_{s}<4 \mu \mathrm{m}\right)$ and accelerates the particles toward the wall for larger particles $\left(D_{s}>8 \mu \mathrm{m}\right)$; in turbulent flow with high Re, small particles are accelerated towards the wall, while larger particles $\left(D_{s}>32 \mu \mathrm{m}\right)$ are lifted away from the wall. This mechanism suggests that the rate of "removal" (or, more precisely, deposition discouragement) is a function not only of flow velocity, but also of particle size.

Another lesser-known force near the wall is called the drainage force, which has its origin in the force required to remove fluid from the region between two approaching surfaces, in this case the particle and the wall [7]. Both this and the lift force are known to be small in a gas but considerably greater in a liquid (which may be why they were not considered in the original Cleaver \& Yates aerosol work [2]). In SLUDGE and TSFOUL. the effect of these forces are conveniently accounted for in empirical constants such as the "removal" constant $A$ and the attachment constant $K_{o}$ (see Equation A.20 in Appendix A). For example, $K_{o}$, which was obtained for small particles $\left(D_{s}<4 \mu \mathrm{m}\right)$ at high $\operatorname{Re}$ values, may encompass not only surface charge effects but also lift effects. However, the dependence on particle size is not captured and, for example, the deposition at high $\mathrm{Re}$ with large particles (i.e., $>32 \mu \mathrm{m}$ ) may then be overestimated.

Whatever the mechanisms for the asymptotic behaviour, comparison with deposit growth in actual SGs suggests that deposit growth does not, in fact, slow down appreciably. It is likely that the "removal" constant $\mathrm{A}$ is nearly zero, and that the deposition is close to linear rather than asymptotic. Also, Equation 8.1 does not explicitly take into account deposit ageing: consolidation of the deposit would harden it, making it resistant to removal. For these reasons, TSFOUL assumes as default value an $A$ value $\left(=3 \times 10^{-12}\right)$ smaller than originally used in SLUDGE $\left(=7 \times 10^{-11}\right.$, as shown in Section A.2), to promote a deposit growth rate that is more in line with growth rates inferred from SG inspections. 
On the other hand, there are other causes, besides removal or re-entrainment, of a declining rate. For example, Bowen and Epstein [8] observed a declining rate of accumulation even when no re-entrainment was observed, and suggested an autoretardation mechanism such as reduced adhesion for the second and subsequent layers of particles. Reduced adhesion may not be a large factor in a SG where both surface-particle and particle-particle interactions involve like charges, and TSFOUL therefore does not account for it. TSFOUL does, however, account for reduced mass flux over time as the flow area decreases from deposit growth (see Section 7.3.7). Another contributor may be reduced particle concentration if the liquid becomes depleted of particles over time. Also, deposition growth may be slowed due to changes in the surface roughness: initially the cavities of a rough surface would readily trap particles, but this deposition eventually smoothes the surface giving both fewer cavities and a thicker viscous boundary layer.

Because of the debate over whether particles are actually removed, it is perhaps more appropriate to call A the deposition "suppression" constant rather than the removal constant, in turbulent flow. It would then represent some of the auto-retardation mechanisms mentioned above that are not explicitly accounted for in TSFOUL.

\subsubsection{Deposition in Laminar Flow}

The SLUDGE code does not take into account differences in deposition behaviour due to a laminar flow regime. The most significant impact of laminar flow is on the "removal" term in the deposition equation (Equation 8.1). The study by Bowen and Epstein [8] on fine particle deposition in laminar flow between parallel plates showed no re-entrainment whatsoever. For laminar flow, TSFOUL therefore uses the same expression as for turbulent flow but for the case of constant A equal to zero:

$$
\delta=\frac{K \rho \phi}{\rho_{s}\left(1-\beta_{s}\right)} t
$$


The growth of deposit would be linear with time, except for indirect causes unrelated to re-entrainment, as discussed previously.

\subsubsection{Change in Particle Concentration}

As deposition occurs, the liquid is depleted of particles because they are left behind on surfaces, but it is also enriched because of evaporation of liquid during boiling. An expression is needed for the change of particle concentration with distance along the channel, i.e., $d \phi / d z$. In a steady-state situation. the change (decrease) in flow rate of particles $\mathrm{dm}_{\mathrm{s}, \mathrm{flow}}$ along an incremental distance $\mathrm{dz}$ along the channel equals the change (increase) in the flow rate of particles $\mathrm{dm}_{\mathbf{5 . d e p}}$ travelling toward the surface to be deposited in the interval dz:

$$
\frac{d m_{s, \text { Aow }}}{d z}=-\frac{d m_{s, d e p}}{d z}
$$

The source term on the left-hand side of Equation 8.3 is a function of the flow area (through the mean diameter $D_{m}$ and channel width $w$ ) and liquid density, and a function of the change in liquid fraction, velocity. and particle concentration over distance :

$$
\frac{d m_{s, \text { flow }}}{d z}=\pi D_{m} w \rho_{f} \frac{d\left((1-\alpha) u_{f} \phi\right)}{d z}
$$

The sink term on the right-hand side of Equation $\mathbf{8 . 3}$ is a function of the surface area, liquid density, liquid fraction, particle concentration, and deposition coefficient $\mathrm{K}$ (which, as defined in Section 8.2, has units of $\mathrm{m} / \mathrm{s}$ and can be viewed as the velocity of the particles toward the surface): 


$$
\frac{d m_{s, d e p}}{d z}=\pi D_{\text {nube }} \rho_{f}(1-\alpha) \phi K
$$

After substituting in Equation $\mathbf{8 . 3}$ and simplifying, we get

$$
\begin{aligned}
& \pi D_{m} w \rho_{f} \frac{d\left((1-\alpha) u_{f} \phi\right)}{d z}=-\pi D_{\text {rube }} \rho_{f}(1-\alpha) \phi K \\
& \frac{d\left((1-\alpha) u_{f} \phi\right)}{d z}=-K(1-\alpha) \phi \frac{D_{r u b c}}{D_{m} w}
\end{aligned}
$$

Separating variables on the left-hand side of Equation 8.7 , we can solve for $\mathrm{d} \phi / \mathrm{dz}$ :

$$
\begin{aligned}
& (1-\alpha) u_{f} \frac{d \phi}{d z}+\phi \frac{d\left((1-\alpha) u_{f}\right)}{d z}=-K(1-\alpha) \phi \frac{D_{\text {tube }}}{D_{m} w} \\
& \frac{d \phi}{d z}=\frac{\phi}{(1-\alpha) u_{f}}\left[-\frac{d\left((1-\alpha) u_{f}\right)}{d z}-\frac{K(1-\alpha) D_{\text {rube }}}{D_{m} w}\right]
\end{aligned}
$$

Note that $(1-\alpha) u_{\mathrm{f}}$ is equivalent to the liquid superficial velocity $j_{\mathrm{f}}$. From the definition of $j_{f}$ (Equation 7.5) and from heat balance (Equation 7.44),

$$
\frac{d\left((1-\alpha) u_{f}\right)}{d z}=\frac{d j_{f}}{d z}=\frac{d j_{f}}{d \chi} \frac{d \chi}{d z}=-\frac{G}{\rho_{f}} \frac{Q \pi D_{t u b e}}{m h_{f g}}
$$

This is then substituted into Equation 8.9, with the assumption that $(1-\alpha) \rho_{\mathrm{f}} \approx \rho$ (see Equation 7.14; the vapour density $\rho_{\mathrm{g}}$ is much lower than the liquid density $\rho_{\mathrm{f}}$ and can therefore be neglected). The final equation becomes 


$$
\frac{d \phi}{d z}=\frac{\phi D_{t u b e}}{u, D_{m} w}\left[\frac{Q}{\rho h_{f g}}-K\right]
$$

In TSFOUL, the particles are deposited on the tube and the support surfaces, and in two channels ( $\mathrm{Y}$ and $\mathrm{Z}$, hence the factor 2 in Equations 8.12a and 8.12b). Also, for turbulent flow (Equation 8.12a), the $\mathrm{K}$ term must be modified to include the "removal" term, i.e., the quantity of particles lost to deposition is lessened if deposition is suppressed. Thus, the difference in particle concentration $\Delta \phi$ in the liquid over interval $\Delta z$ is expressed as the boiling term minus the depletion term (which is a function of regime):

$$
\begin{aligned}
& \Delta \phi_{T}=\frac{\phi D_{\text {rube }} \Delta z}{\mu_{f} A}\left[\frac{Q}{\rho h_{f g}}-2\left(K_{\text {rube }}+K_{\text {tube } / \text { sup }}\right) \exp \left(\frac{-A t\left(u^{-}\right)^{2}}{v_{f}}\right)\right] \\
& \Delta \phi_{L}=\frac{\phi D_{\text {tube }} \Delta z}{\mu_{f} A}\left[\frac{Q}{\rho h_{f g}}-2\left(K_{\text {rube }}+K_{\text {rube/sup }}\right)\right]
\end{aligned}
$$

\subsection{Deposition Coefficient K}

The expression for the overall single-phase deposition coefficient is the same as in Section A.3.1 except that, for horizontal nodes, the coefficients for gravitational and centrifugal settling, $K_{G}$ and $K_{C}$, are added directly to $K_{1 \phi}$ instead of indirectly through the transport $K_{T}$. [This is discussed in more detail in Section 9.5.]

$$
K_{1,}(\text { vertical })=\left[\frac{1}{K_{T}}+\frac{1}{K_{A}}\right]^{-1}
$$




$$
K_{1 \phi}(\text { horizontal })=\left[\frac{1}{K_{T}}+\frac{1}{K_{A}}\right]^{-1}+\left(K_{G} \text { or } K_{C}\right)
$$

where $K_{T}=K_{T H}+K_{D}+K_{l}$

The expression for the overall two-phase coefficient is the same as in Equation A.11:

$$
K_{2 \phi}=K_{1 \phi}+K_{B}
$$

Under both single-phase and two-phase conditions, velocities in the deposition equations are the true velocities in the liquid, i.e., $\mathrm{u}^{*}$ is based on a separated-flow Reynolds number (see Equation 7.8). This accounts for the fact that particles travel with the liquid.

The boiling coefficient $K_{B}$ is also the same as in Equation A.12 (from Asakura et al. [9]):

$$
K_{B}=\frac{B Q}{h_{f s} \rho}
$$

except for the constant $B$ (to be discussed shortly). $K_{B}$ can be obtained by considering that the liquid mass flow rate toward the surface (and hence the flow of particles) equals the evaporation rate under saturated boiling conditions. The flow rate per unit surface area of liquid carrying particles toward the wall is

$$
m_{f, w}=\rho_{f}\left(1-\alpha_{w}\right) u_{f, w}
$$

where the void fraction and liquid velocity are those near the wall, where the boiling is taking place. The rate of evaporation of this liquid per unit surface area is 


$$
m_{\text {evap }}=\frac{Q}{h_{f g}}
$$

Because $m_{f, w}=m_{e v a p}$, we can solve for $u_{f, w}$ :

$$
u_{f, w}=\frac{Q}{\rho_{f}\left(1-\alpha_{w}\right) h_{f g}} \approx K_{B}
$$

This velocity is equivalent to $K_{B}$, except that $K_{B}$ is associated with the bulk conditions (such as particle concentration) and not wall conditions. As the wall conditions are not well-known, an empirical constant B can make up for this, as well as for the slight inaccuracy of setting $\rho_{f}\left(1-\alpha_{w}\right)$ equal to the mean bulk density $\rho$. The resulting equation is the same as Equation 8.17:

$$
K_{B}=\frac{B Q}{h_{f \delta} \rho}
$$

The constant B was determined by Turner \& Godin [10] for magnetite particles depositing onto Inconel-600 under typical SG pressures and temperatures and in alkaline water ( $\mathrm{pH} \mathrm{8.8).} \mathrm{These} \mathrm{B}$ values appeared to be quality-dependent, but not velocitydependent. For TSFOUL, curve-fitting was applied to Turner's data to show their quality dependence. [The threshold value of $\chi=-0.103$ is from the fit, but probably relates physically to the onset of significant void, as described in Section 7.3.2.]

$$
\begin{array}{rlrl}
\chi_{t h} & <-0.103 & B & =0 \\
-0.103<\chi_{t h} & <0 & B & =0.1578 \quad \log _{10}\left(\chi_{t h}+0.15\right)+0.2095 \\
\chi_{t h} & >0 & B & =0.08
\end{array}
$$


The quality dependence of the boiling term reflects the fact that, for subcooled boiling conditions, only part of the heat goes toward the evaporation of liquid (and the associated deposition of particles that the liquid carried). The remainder goes toward heating of the subcooled bulk fluid.

Turner \& Godin [10] found $B$ (and hence $K_{B}$ ) to be an order of magnitude lower in boiling alkaline water ( $\mathrm{pH} 8.5$ to 9.0) than in neutral water, and attributed this to repulsion between the negatively-charged surfaces of magnetite and Inconel-600 at the higher $\mathrm{pH}$. B was also found to be reduced in the presence of chemical dispersants.

\subsection{Models for Particle Transport}

The expression for transport by thermophoresis is same as in Equation A.13. Gravitational and centrifugal settling are discussed in Chapter 9 as they are specific to the supports. Expressions for transport by diffusion and by inertial coasting are explored in the following discussion, and are modified to include entrance effects.

\subsubsection{Deposition from Turbulent Diffusion}

In TSFOUL, the deposition coefficient for diffusion in turbulent flow is based on that of Cleaver and Yates [2], as used in SLUDGE and as expressed in Equation A.16:

$$
K_{D_{\text {surb }}}=0.031 \cdot \cdot\left(\frac{D}{v_{f}}\right)^{2 / 3}
$$

where $D$ is the diffusivity (Equation A.17). 


\section{Comparison using Heat Transfer Analogy}

This equation can be verified by using a heat transfer analogy for the mass transfer of particles [11], with the following assumptions: (1) the particles are small enough that they travel with the flow, and the flow-including its turbulence structure-is not disturbed by their presence, and (2) the particle concentration is low enough that particles do not interact with each other. [Note that these assumptions are valid only for diffusion, and not for other mechanisms such as inertial coasting or settling, for which the particle diverts from the flow path at some point.]

A widely-used correlation for heat transfer in fully-developed turbulent flow in a tube is a variation of the Colburn equation:

$$
N u_{\text {turb }}=0.023 \operatorname{Re}^{08} \operatorname{Pr}^{1 / 3}
$$

To apply the heat/mass transfer analogy, the Nusselt number is replaced by the Sherwood number, and the Prandtl number by the Schmidt number:

$$
S h_{\text {rurb }}=0.023 \operatorname{Re}^{0.8} S c^{1 / 3}
$$

where the Sherwood and Schmidt numbers are defined by:

$$
\begin{aligned}
& S h=\frac{K_{D} D_{h y}}{D} \\
& S c=\frac{\mu_{f}}{\rho_{f} D}=\frac{v_{f}}{D}
\end{aligned}
$$

Equation 8.23 ( $\mathrm{K}_{\mathrm{D} \text {,urb }}$ from Cleaver \& Yates) can be manipulated to have the same form as in Equation 8.25 ( $\mathrm{Sh}_{\text {rurb }}$ based on Colburn's heat transfer). The friction velocity $\mathrm{u}^{*}$ is a 
function of the liquid velocity $\mathrm{u}_{\mathrm{f}}$ and the Darcy friction factor $f$ which, for turbulent flow through smooth tubes, can be expressed as a simple function of Reynolds number:

$$
\begin{aligned}
& u^{\cdot}=u_{f} \sqrt{\frac{f}{8}} \\
& f_{\text {rurb }}=\frac{0.184}{\operatorname{Re}^{0.2}}
\end{aligned}
$$

Substituting in Equation 8.23 for these parameters, we obtain

$$
K_{D, \text { furb }}=0.031 u_{f}\left(\frac{0.184}{8 \operatorname{Re}^{0.2}}\right)^{1 / 2}\left(\frac{D}{v_{f}}\right)^{2 / 3}
$$

Maltiplying each side of the equation by $D_{\text {hy }} / D$, and top and bottom by $v_{f}$, yields

$$
S h_{\text {turb }}=0.0047 \operatorname{Re}^{-01} \frac{u_{f} D_{h y}}{v_{f}}\left(\frac{v_{f}}{D}\right)^{1 / 3}
$$

Substituting for $\operatorname{Re}$ and $\mathrm{Sc}$ gives

$$
S h_{\text {rurb }}=0.0047 \operatorname{Re}^{0.9} S c^{1 / 3}
$$

This equation is similar in form to that obtained in Equation 8.25 from the heat transfer analogy, with only the constant and Reynolds number exponent differing. Considering the relatively high Schmidt numbers in effect for diffusing particles in the SG (typically $S c \approx 10^{4}$ ), better agreement could be obtained by using a more applicable heat transfer correlation than the Colbum one, e.g., that of Petukhov [12] which is valid for high Pr and hence Sc numbers [11]. 


\section{Entrance Effects}

Equation 8.23 does not account for entrance effects. To develop this, the heat/mass transfer analogy can be used. For a sharp-edged entrance, as in the broached plate geometry, a convenient correlation for the average heat transfer coefficient over the entrance region is [13]

$$
h_{\text {ove }}=h_{\infty}\left[1+\left(\frac{D_{h y}}{z}\right)^{0.7}\right]
$$

A local value is needed rather than the average value, and can be obtained from the difference in heat transferred between the interval $z$ and the interval $z+\delta z$ :

$$
h_{\text {loc }=} \delta z \pi D_{w}\left(T_{w}-T_{b}\right)=\left[h_{\text {ove }=+\delta}(z+\delta z)-h_{a v e, z} z\right] \pi D_{w}\left(T_{w}-T_{b}\right)
$$

or $\quad h_{\text {locin }} \delta z=h_{\text {ove } z+\varepsilon z}(z+\delta \varepsilon)-h_{\text {ove } z} z$

where $\pi D_{w}$ is the surface circumference, and $T_{w}-T_{b}$ is the temperature difference between wall and bulk fluid; these parameters are assumed to remain constant. Dividing Equation $8.34 b$ through by $\delta z$ gives

$$
h_{\text {loc: }:=} h_{\text {ove } z+\dot{\alpha}}+\left(\frac{h_{\text {ove }, z+\varepsilon}-h_{\text {ove }, \tilde{z}}}{\delta \varepsilon}\right) z
$$

As $\delta z$ approaches zero, the term in brackets in Equation 8.35 becomes a derivative; making use of Equation 8.33, the local value of $h$ becomes 


$$
\begin{aligned}
& h_{\text {loc, },=}=h_{\text {ave }, z}+\frac{d h_{\text {ove } z}}{d z} z \\
& h_{\text {loc } z=}=h_{\infty}\left[1+0.3\left(\frac{D_{h y}}{z}\right)^{0.7}\right]
\end{aligned}
$$

The equivalent expression for the local Nusselt number is

$$
N u_{l o c ;}=N u_{\infty}\left[1+0.3\left(\frac{D_{h y}}{z}\right)^{0.7}\right]
$$

The Nusselt number is analogous to the Sherwood number, defined in Equation 8.26. It follows then, with the fully-developed $K_{D . t u r b}$ defined in Equation 8.23, that

$$
K_{D, \text { rurb }}(\text { local })=K_{D, \text { rurb }}\left[1+0.3\left(\frac{D_{h y}}{z}\right)^{0.7}\right]
$$

The greatest value of $K_{D}$ is at the start of the flow development (at very small $z$ ), where the flow boundary layer is very thin and the particles can diffuse readily to the wall. The application of the entrance effect to support deposition is discussed in Section 9.4.

\subsubsection{Deposition from Laminar Diffusion}

For laminar flow, TSFOUL uses an expression for mass-transfer controlled deposition for suspensions flowing in a rectangular channel from Vasak et al.[14] which, in turn, is based on work by Bowen et al.[15]. 


$$
K_{D . \mathrm{lam}}(\text { local })=\frac{1}{0.893}\left(\frac{M D^{2}}{9 z}\right)^{1 / 3}
$$

where $\mathrm{z}$ is the axial distance from the start of flow development. [Unlike the Cleaver \& Yates expression for $K_{D, t u r b}$ in Equation 8.23, this expression already accounts for entrance effects.] Equation 8.40 dictates that $K_{D . l a m}$ decreases as flow develops with increasing $\mathrm{z}$, and approaches zero as $\mathrm{z}$ becomes very large. It would be more realistic if $\mathrm{K}_{\mathrm{D} \text { lam }}$ were to reach a constant value. However, Equation 8.40 was obtained for a channel entirely within the development length, which is also the case for SG support channels.

$M$ is a function (with units of $s^{-1}$ ) related to the slope of the fully-developed velocity distribution in a rectangular channel of width $2 b$ and length $2 a$ [14]:

$$
M=\frac{3 u_{f}}{b} \frac{1-\frac{2}{\pi^{2}} \sum_{n=1}^{\infty} \operatorname{sech}[(n-1 / 2) \pi a / b] /(n-1 / 2)^{2}}{1-\frac{6}{\pi^{5}} \frac{b}{a} \sum_{n=1}^{\infty} \tanh [(n-1 / 2) \pi a / b] /(n-1 / 2)^{5}}
$$

\section{Comparison using Heat Transfer Analogy}

Equation 8.41 is an awkward expression that does not shed much light on the diffusion process. For comparison, an approximate expression for $\mathrm{K}_{\mathrm{D}}$ in laminar flow can be determined, again using a heat transfer analogy for the mass transfer of particles [11]. Grouping the constants in Equation 8.40 and Equation 8.41 together, knowing that $\mathrm{a}=5.7$ $\mathrm{mm}$ and $\mathrm{b}=2.2 \mathrm{~mm}$ for a rectangular slot representing a flow channel in the Bruce-type broached plate, we obtain 


$$
K_{D, l a m}(\text { broach })=0.844\left(\frac{u_{f} D^{2}}{b z}\right)^{1 / 3}
$$

This is now in a convenient form for comparison. The average heat transfer in developing laminar flow in a tube can be described by the well-established empirical equation of Sieder-Tate for developing flow in a circular tube (see [16]):

$$
N u_{\text {ave }}=1.86\left(\operatorname{Re} \operatorname{Pr} D_{h y} / z\right)^{1 / 3}
$$

This equation is valid for $\left(\operatorname{Re} \operatorname{Pr} D_{h y} / z\right)>10$. The viscosity correction factor for large wall-to-bulk temperature differences usually included in the Sieder-Tate equation is omitted here, because this difference is relatively small. As in Section 8.1.5, the Nusselt and Prandtl numbers are replaced by their mass-transfer equivalents. The equation for the average mass transfer in developing laminar flow in a tube is then

$$
S h_{a v e}=1.86\left(\operatorname{Re} S c D_{h y} / z\right)^{1 / 3}
$$

The Sherwood and Schmidt numbers are defined in Equations 8.26 and 8.27. The above equation gives the average Sherwood number, and thus the mass transfer coefficient over the total length $\mathrm{z}$, while the local mass transfer coefficient is required in TSFOUL in each node. It can be shown, using the same approach as in Equation 8.34b, that the local Nusselt number is $2 / 3$ that of the average $\mathrm{Nu}$; by analogy, the local value of $\mathrm{Sh}$ is

$$
S h_{l o c}=\frac{2}{3} S h_{\text {ave }}=1.24\left(\operatorname{Re} S c D_{h y} / z\right)^{1 / 3}
$$

This equation is valid for a circular tube. As the equivalent hydraulic diameter concept is not as applicable in laminar flow as it is in turbulent flow, some adjustment must be made for the rectangular geometry that is used to model the support flow channel. One method 
is to compare multiplying factors for heat transfer with different geometries. According to Table 6.1 in [16], the factor for heat transfer at constant temperature (equivalent to constant concentration at the wall, for mass transfer) for a circular tube is 3.657 ; for a rectangular channel with thickness-to-width ratio of about $1 / 2.6$, which would represent the Bruce-type broached plate, it is approximately 3.70. As this is very close to 3.657 , Equation 8.45 happens to be applicable as is. Equation 8.26 is now used to express this in terms of the deposition coefficient:

$$
K_{D . l a m}=1.24 \frac{D}{D_{h y}}\left(\operatorname{Re} S c D_{h y} / z\right)^{1 / 3}
$$

To convert this equation into a form that can be compared with that of Vasak (see Equation 8.40), the Reynolds and Schmidt definitions are used:

$$
\begin{aligned}
K_{D, \text { lam }} & =1.24 \frac{D}{D_{h y}}\left(\frac{\rho_{f} u_{f} D_{h y}}{\mu_{f}} \frac{\mu_{f}}{\rho_{f} D} \frac{D_{h y}}{z}\right)^{1 / 3} \\
& =1.24\left(\frac{u_{f} D^{2}}{D_{h y} z}\right)^{1 / 3}
\end{aligned}
$$

For an annulus, $D_{h y}=2 \mathrm{w}$, but for a slot of the broached plate dimensions, $D_{h y}=1.44 \mathrm{w}=$ 2.88 b. Equation 8.48 can therefore be re-written as

$$
K_{D, l a m}(\text { broach })=0.872\left(\frac{u_{f} D^{2}}{b z}\right)^{1 / 3}
$$

which is very close to Vasak's correlation as expressed in Equation 8.42. 


\subsubsection{Deposition from Inertial Coasting}

TSFOUL uses the same expression for $K_{1}$ as in SLUDGE, as shown in Equation A.14. However, some subtle changes were made. Papavergos and Hedley [17] demonstrated that diffusion and inertial coasting are mutually exclusive regimes, with diffusion being a function of Schmidt number Sc (as in the Cleaver-Yates expression, Equation A.16), and inertial coasting being a function of particle relaxation time $\mathrm{t}_{\mathrm{p}}{ }^{+}$(defined in Equation A.15). [A third regime, called impaction, would occur at high values of $t_{p}{ }^{+}$and its nondimensionalized coefficient $K_{\text {imp }} / u^{*}$ is constant.] Thus,

$$
\begin{array}{ll}
K_{D} / u^{\cdot} \alpha(S c)^{-2 / 3} & \text { at } t_{p}^{+}<02 \\
K_{l} / u^{\cdot} \alpha\left(t_{p}^{+}\right)^{2} & \text { at } 0.2<t_{p}^{+}<20 \\
K_{\text {ump }} / u^{*} \text { const. } & \text { at } t_{p}^{+}>20
\end{array}
$$

The upper limit of the inertial coasting regime could be obtained simply by calculating $\mathrm{K}_{\mathrm{i}}$ with $t_{p}{ }^{+}=20$. However, in TSFOUL, this gives a value of $K_{\mathrm{I}}$ that is unrealistically large i.e., under conditions where inertial coasting is a key mechanism, deposits are then predicted to become thick after only a few months . Papavergos and Hedley showed that the values of $K_{I}$ at the limits of the inertial coasting regime are typically about 4 orders of magnitude apart; based on this and on typical $K_{D}$ values from the TSFOUL calculations (which gives the lower limit), an upper limit for $K_{l}$ at $t_{p}{ }^{+}=7$ was found to be more appropriate in TSFOUL. This is also more in line with the limit of $\mathrm{t}_{\mathrm{p}}{ }^{+}=0.1$ to 0.2 determined by Turner (see Section A.4.2). [The difference between the Papavergos limit and that of Turner may be due to different interpretations or definitions of the inertial coasting mechanism [18].]

Recognizing that the transition between the diffusion and inertial coasting regimes needs to be smooth, the smoothing technique used in Equations 7.11 and 7.20 for the friction factor is also applied here: 


$$
K_{D I}=\left(\boldsymbol{K}_{D}^{3}+\boldsymbol{K}_{I}^{3}\right)^{1 / 3}
$$

\section{Entrance effects}

An entrance effect is postulated for $\mathrm{K}_{\mathrm{l}}$, because a thinner boundary layer in the developing region would allow particles to coast to the surface more easily. For now, TSFOUL uses the same expression as for diffusion in turbulent flow (see Equation 8.39), although the heat/mass transfer analogy assumed in this expression is not as applicableparticles do not follow the flow during their coasting.

$$
K_{t}(\text { local })=K_{I}\left[1+0.3\left(\frac{D_{h y}}{z}\right)^{0.7}\right]
$$

The greatest value of $K_{1}$ is at the start of the flow development (at very small $z$ ), where the flow boundary layer is very thin and the particles can coast readily to the wall. The application of the entrance effect to support deposition is discussed in Section 9.4.

\subsection{Model for Particle Attachment}

The expression for the attachment deposition coefficient $\mathrm{K}_{\mathrm{A}}$ is similar to that in Equation A.20, in that it shows the same strong dependence on activation energy and surface temperature. The original expression was obtained for the attachment of magnetite particles to an Inconel-600 surface, under high temperature and alkaline conditions [10]. Turner subsequently determined that such an expression was suited to laminar-flow conditions only and that, at higher flow rates, the particles would have enough kinetic energy to overcome the repulsive forces [19]. In other words, under laminar conditions in the $\mathrm{SG}, \mathrm{K}_{\mathrm{A}}$ is independent of velocity, but under turbulent conditions the attachment 
improves as the kinetic energy (which is a function of velocity) becomes greater than the repulsive energy barrier.

The particle kinetic energy in the bulk of a turbulent flow was obtained from work by Yung et al. [20]; Tumer then adapted this for particles at an approximate distance of $D_{s} / 2$ from the wall, where surface forces are important [19]. For such a particle,

$$
K E_{\text {turb }}=\frac{3 \pi \rho_{f} D_{s}^{4}}{v}\left(\frac{u^{\bullet}}{29}\right)^{3}
$$

The effect of the fluid velocity on $\mathrm{K}_{\mathrm{A}}$ is then determined by replacing the thermal energy $k T$ with the total energy $\left(k T+K E_{\text {turb }}\right)$ :

$$
K_{d}=K_{o} \exp \left(\frac{-E_{a} / N}{k T_{w}+K E_{\text {turb }}}\right)=K_{o} \exp \left(\frac{-E}{k T_{w}+K E_{\text {turb }}}\right)
$$

where $K_{o}=5.697$ and $E_{a}=76.4 \mathrm{~kJ} / \mathrm{mol}$, as with the original Equation A.20. The activation energy $E_{a}$ is divided by Avogadro's number $\mathrm{N}\left(=6.022 \times 10^{23} \mathrm{molecules} / \mathrm{mol}\right)$, to work on a molecular basis when the Boltzmann constant $\mathrm{k}\left(=1.3807 \times 10^{-23} \mathrm{~J} / \mathrm{K}\right.$ per molecule) is in the equation. Note that $E=E_{a} / N=E_{a} k / R$.

In considering attachment, many researchers have modeled the opposite dependence on velocity. For example, Vasak et al. [14] described the attachment of neutral particles in laminar flow to be an inverse function of $\left(u^{*}\right)^{2}$, i.e., there is less attachment as the velocity increases. This is a "shearing" or "scouring" concept, akin to the removal or suppression described in Section 8.1, and is therefore unrelated to the kinetic energy concept described above.

Note that the activation energy determined for Inconel-600, which is a typical SG tube material, would be similar for carbon steel or stainless steel, which are typical SG support 
materials. In all these cases, both the surface and the magnetite particles are negatively charged, under alkaline $\mathrm{pH}$ conditions [19].

The next Chapter describes the deposition models in TSFOUL that are specific to the support.

\section{References}

[1] Y. Liner, M.B. Carver, C.W. Turner, and A.O. Campagna, "Simulation of Magnetite Particulate Fouling in Nuclear Steam Generators", Proceedings of the ASME meeting on Steam Generator Sludge Deposition in Recirculating and Once Through Steam Generator Upper Tube Bundle and Support Plates, NE-Vol.8, Book No. G00690, 1992.

[2] J.W. Cleaver and B. Yates, "A Sub-Layer Model for the Deposition of Particles from a Turbulent Flow", Chemical Engineering Science, Vol.30, pp.983-992 (1975).

[3] N. Epstein, "Fouling in Heat Exchangers", Proceedings of the 6th Int. Heat Transfer Conference, Vol.7, 1978 August 7-11.

[4] P. Adomeit and U. Renz, "Deposition of Fine Particles from Aqueous Suspensions", Proceedings of the 10th Int. Heat Transfer Conference, Vol.5, Brighton, U.K., 1994 August 14-18. 
[5] P.O. Rouhiainen and J.W. Stachiewicz "On the Deposition of Small Particles from Turbulent Streams", Journal of Heat Transfer, Transactions of the ASME, pp.169-177 (1970 February).

[6] P.G. Saffman, "The Lift on a Small Sphere in a Slow Shear Flow", Journal of Fluid Mechanics, Vol.22, Part 2, pp.385-400 (1965).

[7] H. Brenner, "The Slow Motion of a Sphere through a Viscous Fluid towards a Plane Surface, Chemical Engineering Science, Vol.16, pp.242-251 (1961).

[8] B.D. Bowen and N. Epstein, "Fine Particle Deposition in Smooth Parallel-Plate Channels", Journal of Colloid and Interface Science, Vol.72, No.1, pp.81-97 (1979 October).

[9] Y. Asakura, M. Kikuchi, S. Uchida, and H. Yusa, "Deposition of Iron Oxide on Heated Surfaces in Boiling Water", Nuclear Science \& Engineering, Vol.67, pp.17 (1978).

[10] C.W. Turner and M. Godin, "Mechanisms of Magnetite Deposition in Pressurized Boiling and Non-Boiling Water", Proceedings of the 2nd Int. Conf. on Steam Generators \& Heat Exchangers, Toronto, Canada, 1994 June 13-15.

[11] Memo from J.T. Rogers to H. Rummens, "Some Thoughts on Particulate Deposition in Tube Support Plate Clearances", 1996 July 19.

[12] B.S. Petukhov, "Heat Transfer and Friction in Turbulent Pipe Flow with Variable Physical Properties", in Advances in Heat Transfer, J.P. Harnett and T.F. Irvine (editors), Academic Press, New York, 1970, pp. 505-564. 
[13] W.H. McAdams, Heat Transmission. $3^{\text {rd }}$ Edition, McGraw-Hill, 1954.

[14] F. Vašák, B.D. Bowen, C.Y. Chen, F. Kaštánek, and N. Epstein, “Fine Particle Deposition in Laminar and Turbulent Flows", Canadian Journal of Chemical Engineering, Vo.73, pp.785-792 (1995 December).

[15] B.D. Bowen, S. Levine, and N. Epstein, "Fine Particle Deposition in Laminar Flow Through Parallel-Plate and Cylindrical Channels", Journal of Colloid and Interface Science, Vol.54, pp.375-390 (1976).

[16] J.P. Holman, Heat Transfer, $7^{\text {th }}$ Edition, McGraw-Hill, Inc., 1990.

[17] P.G. Papavergos and A.B. Hedley, "Particle Deposition Behaviour from Turbulent Flows", Review Paper. Chemical Engineering Research \& Design, Vol.62, pp.275-295 (1984 Sept).

[18] C.W. Turner, private communication, 1999 February.

[19] C.W. Turner, private communication, 1996 January.

[20] B.P.K. Yung, H. Merry, and T.R Bott, "Mechanisms of Deposition and Reentrainment of Particulate Fouling in an Aqueous System", Proceedings of the UK $2^{\text {nd }}$ National Heat Transfer Conference, 1988, p. 1689. 


\section{Chapter 9}

\section{TSFOUL: SUPPORT DEPOSITION MODELS}

The following models, based primarily on the proposed fouling mechanisms of Figure 6.4 , were implemented as specific to deposition on supports:

- impaction of large particles onto the bottom of the support,

- centrifugal settling in the vena contracta region,

- consolidation of existing deposits due to flashing and precipitation of cementing agents, especially at the support inlet,

- enhanced deposition after the boundary layer re-attachment within the support,

- gravitational or centrifugal settling on the top of the support, and

- differential deposition in unequally-sized flow channels.

\subsection{Particle Impaction on Support Bottom}

As shown in Figure 9.1, particles collect on the upstream (bottom) support face because inertia has caused them to travel straight instead of following the curving flow stream. Whether the particles stay in place or bounce away is discussed later in terms of a sticking probability. The fraction of particles impacting (i.e., contacting) the surface is a function of mainly particle size, but also flow velocity and physical properties. Very small particles will follow the flow and never impact; with larger particles, all may impact.

Studies by Ranz and Wong [1] provide impaction efficiencies for various geometries, for particles entrained in aerosols. The impaction efficiency on a flat surface is zero if no particle in the flow stream directed toward it impacts and unity if all particles impact. The efficiency is a function of a non-dimensional inertia parameter $\Psi$ : 


$$
\Psi=\frac{C \rho_{s} u_{f} D_{s}^{2}}{18 \rho_{f} v_{f} w_{j e t}}
$$

where $w_{j e t}$ is the width of the oncoming jet of fluid, and $C$ is a non-dimensional empirical correction factor for resistance of a gas to movement of small particles (i.e., a drag coefficient) [1]:

$$
C=1+\frac{2 L}{D_{s}}\left[1.23+0.41 \exp \left(-0.44 \frac{D_{s}}{L}\right)\right] \text { for } 0.1<\frac{2 L}{D_{s}}<134
$$

where $\mathrm{L}$ is the mean free path of the fluid molecule, and equals $2 \mu / \mathrm{pu}$.

Note that Equation 9.1 is based on Stokes' Law (as is the expression for centrifugal settling-see Equation A.19-where the radius of curvature is used instead of the parameter $w_{j e t}$, and the dependence on $u_{f}$ is squared instead of linear). $\Psi$ is the ratio of the force necessary to stop a particle initially traveling at velocity $u_{f}$ in the distance $w_{j e t} / 2$, to the fluid resistance at a relative particle velocity of $u_{\mathrm{f}}$. It can also be seen as the ratio of the stopping distance-i.e., the distance a particle will penetrate into still fluid when given an initial velocity of $u_{\mathrm{f}}-$ to the diameter of the surface or jet width.

It is assumed that these relationships and their empirical constants can be applied to micron-sized magnetite particles in water. ${ }^{8}$ For a rectangular jet impacting on a plate, impaction efficiencies $N_{\text {imp }}$ were approximated from experimental aerosol data [1] as follows:

$$
\begin{array}{ll}
\text { if } \sqrt{ } \Psi<0.32 & N_{\text {imp }}=0 \\
\text { if } 0.32 \leq \sqrt{ } \Psi \leq 0.80 & N_{\text {imp }}=m(\sqrt{ } \Psi-0.32), \text { where } m=1 /(0.80-0.32) \\
\text { if } \sqrt{ } \Psi>0.80 & N_{\text {imp }}=1
\end{array}
$$

8 This assumption was also used in applying Cleaver and Yates' aerosol correlations [2] in the SLUDGE code, as discussed in Appendix A. The success of the heat/mass transfer analogy discussed in Sections 8.3.1 and 8.3.2 gives some confidence that the assumption holds. 
Even if a particle has sufficient mass and velocity to impact the surface, it may not stay there. In general, the "sticking" probability $\mathbf{N}_{\mathbf{s t}}$ is unity for well-adhering particles but for poorly-adhering particles (which are typical of the SG because both the support surface and the magnetite particles are negatively charged), sticking is roughly proportional to the inverse of the shear stress $\tau_{w}$ at the wall $[3,4]$ :

$$
N_{s t} \approx \frac{\tau_{\min }}{\tau_{w}}
$$

If $\tau_{w} \leq \tau_{\min }$ then $\mathbf{N}_{\mathrm{st}}=1$. The parameter $\tau_{\min }$ is the shear stress below which all impacted particles will stick, and is given the value 0.1 ;. This remains approximate until the relationship between $N_{S}$ and $\tau_{w}$ is better understood. $\tau_{w}$ is based on the liquid transverse velocity along the upstream surface of the support. [In this model, it is assumed that the axial velocity brings the particles to the surface and the transverse velocity removes them, thereby avoiding the "conceptual problem" discussed in Section 8.1.1.] This transverse velocity is difficult to establish accurately, but Figure 4.12 suggests that the region below the support has velocities several times lower than the mainstream. The transverse velocity is therefore taken as $1 / 3$ that of the axial velocity $u_{\mathrm{f}}$. Hence,

$$
\tau_{w}=f \frac{\rho_{f}\left(u_{f} / 3\right)^{2}}{8}
$$

The net impaction deposition coefficient $K_{F}$ (for which subscript $F$ refers to "filter") on the upstream surface is then simply a function of the oncoming particle velocity-which is assumed to equal the fluid velocity - times the fractions of particles that impact and stick:

$$
K_{F}=u_{f} N_{s m p} N_{s s}
$$


This coefficient is used in the calculation of deposit thickness. This deposit is then made subject to "suppression" forces. In turbulent flow, this would be dictated by the empirical suppression factor A. However, this constant is not quite appropriate for the bottom of the support as it allows unrealistically thick deposits.

An empirical limit to the deposit thickness was therefore imposed. ${ }^{9}$ The critical loading was estimated to occur when the deposit thickness is $1 / 2$ the surface width (i.e., the width of the support "annulus" facing the flow). This is based on flow visualization experiments in which $1-\mathrm{mm}$ metallic flakes formed deposits on the upstream face (see Section 4.3.4); once this surface was "fully loaded" no further deposition occurred.

$\mathrm{K}_{\mathrm{F}}$ has its own accounting for "sticking" and is therefore not a classic transport term subject to attachment. [Another way of viewing this is to consider that the dynamic forces of the flow impinging on the horizontal surface will likely overcome particlesurface repulsive forces.] For the upstream horizontal surface of the support (Node 20), the overall deposition coefficient is therefore simply:

$$
K_{1 \text {, sup }}(i=20)=K_{F}
$$

This equation applies only to the upstream horizontal surface (Node 20); $K_{F}$ is zero for all other surfaces. This surface area is considered variable, i.e., as deposits grow at the broach hole inlet, the surface area for $K_{F}$ increases.

Impaction of large particles appears to be a dominant mechanism in the lower regions of the SG close to the feedwater inlet and tube sheet, which typically feature thick, coarse deposit layers on the underside. The modeling of this phenomenon would benefit from a more accurate expression for the sticking probability $\mathrm{N}_{S}$, and better estimates of the transverse velocity and hence shear stress.

\footnotetext{
9 In reality, $N_{\mathrm{imp}}$ and $\mathrm{N}_{\mathrm{st}}$ are reduced to zero as the surface shape changes from perpendicular to the flow (clean support) to parallel to the flow ("fully loaded" support).
} 


\subsection{Deposition in Vena Contracta Region}

As shown in Figure 6.1, the vena contracta region at the support inlet typically includes a small recirculation zone. The model for centrifugal settling was therefore applied in TSFOUL to the nodes in this region. Here, the deposition coefficient $\mathrm{K}_{\mathrm{V}}$ is expressed as:

$$
K_{\nu^{-}}=\frac{\left(\rho_{s}-\rho_{f}\right) u_{f, \text { vena }}^{2} k_{c, \text { vena }} D_{s}^{2}}{18 \rho_{f} v_{f}}
$$

The streamline curvature $k_{c, v e n a}$ is the inverse of the radius $\left(k_{c}=1 / R\right)$, which is taken as $\sim 1 / 4$ the length of the vena contracta region, based on the experimental results shown in Figure 4.12. The local velocity $u_{\mathrm{f}, \text { vena }}$ is taken as $\sim 1 / 10$ that of the mainstream velocity $u_{\mathrm{f}}$, as a crude estimate from observation and from measurements of recirculation velocities above the support (see Figure 4.12).

The full $\mathrm{K}_{\mathrm{V}}$ value is applied only at the peak flow contraction: it is made to follow a sine shape such that $K_{V}$ peaks just beyond the entrance of the support, and falls to zero at the estimated point of boundary layer re-attachment $\left(z_{i}=z_{a n t}\right)$ :

$$
K_{v^{\prime},}=K_{r} \cdot \sin \left(\pi \frac{z_{1}}{z_{a t r}}\right)
$$

This sine shape approximates the expected shape of the boundary layer on the support in the vena contracta region, as shown in Figure 6.1.

Not all particles in the flow are subject to becoming "trapped" in the recirculation eddy. For example, while Equation 9.8 shows that $K_{v}$ increases with the square of the particle diameter $D_{s}$, given a situation in which the flow is generally parallel to the surface, most large particles will have sufficient momentum to flow past the eddy. Also, perhaps only 
those particles that would have otherwise traveled through the cross-sectional area occupied by the separated boundary layer would be involved. This leads to a further modification:

$$
K_{v, s}(n e t)=K_{V s}\left(1-N_{s m p}\right)\left(1-C_{c}\right) N_{s t}
$$

The ( $1-\mathrm{N}_{\text {imp }}$ ) term is borrowed from the impaction model (Section 9.1), and reflects the fact that those particles most likely to impact in a perpendicular geometry (such as the bottom of the support) will be least likely to impact in a parallel one. The $\left(1-C_{c}\right)$ term is borrowed from sudden-contraction pressure loss (Section 7.3.5), and represents the fraction of cross-sectional flow area that is occupied by the recirculation region; this is taken to (approximately) equal the fraction of the particles that would be subject to deposition. The $\mathrm{N}_{\mathrm{st}}$ term, as in the impaction model, determines what fraction of the impacted particles actually stick; here, it is calculated from a shear stress (see Equation 9.5) based on a reduced local velocity $u_{f, v e n a}=u_{f} / 10$ (as discussed after Equation 9.8) . These terms are somewhat speculative but are necessary to avoid an unrealistically high deposition rate in the vena contracta region.

The velocity reduction is also applied to the friction velocity $u^{*}$, i.e., $u_{\mathrm{f}}$ in Equation A.5 is replaced by $\left(u_{\mathrm{f}} / 10\right)$, which is then used in the calculation of deposition coefficients and deposit thickness, on the support and in the vena contracta region only.

Because it uses its own accounting for sticking, $K_{V}$ is not subject to the classic attachment model and is therefore treated independently. [Another way of viewing this is to consider that centrifugal forces would help to overcome particle-surface repulsive forces.]

$$
K_{1,}=\left[\frac{1}{K_{T}}+\frac{1}{K_{A}}\right]^{-1}+K_{V}
$$


Because the deposition occurs on a surface parallel to the flow, the classic $K_{T}$ and $K_{A}$ combination from Equation A.8 is included; in practice, this combination is reduced to zero because very little thermophoresis, diffusion, or inertial coasting occurs through the thick boundary layer in the vena contracta region.

Centrifugal settling would be a dominant mechanism at the inlet of the support, which typically features a lip of deposit. The modeling of this phenomenon would benefit from a better expression for the fraction of particulates in the flow that become trapped within a vena contracta region.

\subsection{Consolidation due to Flashing}

Solubles in a saturated solution will precipitate on boiling surfaces (as discussed in Section 6.2). They will also precipitate during pressure reductions, due to flashing of thermally-saturated liquid to vapour, as illustrated in Figure 9.2. Solubles such as iron and copper are known to be deposit cementing agents, as they are typically found in the pores of very hard deposits. Impurities such as silicates and calcium salts, which find their way into the SG feedwater from condenser leaks ${ }^{10}$, also harden existing deposits [5].

In TSFOUL, the effect of this precipitation is modeled as reduction in the suppression factor $\mathrm{A}$ with the reduction in pressure along the channel, i.e., a weakening of the "removal" process due to consolidation or hardening of the deposit. Where the local pressure is lowest, i.e., at the support inlet as illustrated in Figure 5.6a, the consolidation is expected to be greatest as well due to the greatest extent of precipitation of cementing agents. To capture this, a simple model was developed for TSFOUL which allows $A$ to vary axially.

10 Leakage occurs because of a higher pressure in the condenser tubes compared to the secondary-side exhaust steam coming from the turbines. 
It is assumed, in this model, that $\mathrm{A}$ is an inverse exponential function of the weight fraction of precipitate in the deposit. Consolidation studies with artificial sludges [5] showed that only 1-2\% of an impurity was required to significantly harden the sludge (and the sludge generally became harder under heat transfer conditions than in an adiabatic environment). An empirical function was based on this, as shown in Figure 9.3: with no precipitate (fraction $F=0$ ), $A=3 \times 10^{-12}$, as discussed in Section 8.1.1. With 1\% precipitate (fraction $\mathrm{F}=0.01$ ) $\mathrm{A}=3 \times 10^{-17}$ which is essentially zero:

$$
A_{1}=A_{o} \exp (-1150 F)
$$

where $A_{0}=3 \times 10^{-12}$ as the value of $A$ with no consolidation.

An expression for $\mathrm{F}$ is now required. This parameter can be determined as the ratio of the mass of precipitate to the mass of particulate deposited, in a given node and time period. To get the mass of precipitate, it is assumed that the liquid is saturated in the soluble species. After a pressure reduction, flashing results from a change in the liquid enthalpy. Conservation of mass and energy dictate that the increase in the gas flow rate or energy equals the decrease in liquid mass flow rate or energy. From one node $i-1$ to the next node i,

$$
\begin{aligned}
& m_{g, d}+m_{f, d}=m_{g, d-1}+m_{f, d-1} \\
& m_{g, d} h_{g, d}+m_{f, d} h_{f, d}=m_{g, d-1} h_{g, t-1}+m_{f,-1} h_{f, d-1}
\end{aligned}
$$

The unknowns are $m_{g, i}$ and $m_{f, i}$. The equations can be simplified, with $h_{g}-h_{f}=h_{f g}$, and with $h_{g, i}=h_{g, i-1}$ (because the vapour enthalpy is fairly insensitive to pressure). The increase in mass flow rate of the vapour for node $i$ is then:

$$
\Delta m_{g, d}=m_{g, d}-m_{g,-1}=m_{f,-1}\left(\frac{h_{f,-1}-h_{f, l}}{h_{f g . t}}\right)
$$


Given the concentration of solubles $\phi_{c}$ (in $\mathrm{kg}$ solubles per $\mathrm{kg}$ liquid), the corresponding rate of precipitation in $\mathrm{kg} / \mathrm{s}$ in node $\mathrm{i}$ is then:

$$
S_{c, d}=\Delta m_{g,} \phi_{c}
$$

where the subscript $\mathrm{c}$ refers to the soluble "cementing agent". [A more exact approach would be to take the difference between the bulk concentration $\phi_{c, b}$ and the solubility limit concentration $\phi_{c, w}$ at the wall temperature.] The "flow rate" of solid particles (subscript s) through node $i$ is

$$
S_{s, b}=\boldsymbol{m} \phi_{s, s}
$$

where $m$ is the mass flow rate of the fluid and $\phi_{s}$ is the concentration of the particles in the fluid.

The ratio of "flow rates" of precipitate to particulate in the fluid could be seen as equivalent to the ratio of masses of precipitate to particulate in the deposit (i.e., fraction F), except for one factor: deposition for precipitate is more likely, because these cementing agents are known to enter the pores of existing deposits readily. ${ }^{1}$ In other words, deposition of the precipitate would be governed by transport only, or $\mathrm{K}_{\mathrm{T}}$. while deposition of the particles would be governed by transport and attachment, or $\mathrm{K}_{\mathrm{fot}}=$ $\left(1 / K_{T}-1 / K_{A}\right)^{-I}$. A correction factor of $K_{T} / K_{t o t}=1+K_{T} / K_{A}$ is therefore applied, in which the $K_{T}$ and $K_{A}$ values are taken to be those for the tube surface (for convenience). Thus,

$$
F=\frac{S_{c}}{S_{s}}\left(1+\frac{K_{T}}{K_{A}}\right)
$$

$"$ For example, microscopic cross-sections of deposits from copper-bearing SG units typically reveal threads of metallic copper throughout the deposit. 
This expression assumes that the full mass of precipitate in one node enters the deposit in that same node. This is not realistic, as the fluid will sweep the precipitate along. To somewhat compensate for this, for the vena contracta region-where the inlet pressure reduction is expected to be largest and the fluid velocity highest - the mass of precipitate from the first node is spread over all the nodes: $F_{\text {vena }}=F_{\text {sup,inje }} /$ \# vena nodes.

F can now be entered into Equation 9.12 for an updated value of $A$, after which the calculations of deposit thicknesses are repeated. The local A value is applied to both tube and support surfaces, as precipitates from the bulk fluid would get deposited on both surfaces. Note that the quantity of deposit from precipitate is very small compared to the particulate deposit, and is therefore not added to any deposit volume.

Flashing would occur at any location in the SG where the liquid is thermally saturated and a pressure loss occurs. [Also, precipitation of solubles would occur at any location in the SG where the liquid is evaporating.] The mechanism of precipitation of cementing agents as a result of flashing would be at its strongest at the inlet of the support, where hard lips of deposit have indeed been found in the SG. The modeling of this phenomenon would benefit from a better understanding of the relationship between the quantity of cementing agent and the subsequent deposit growth.

\subsection{Enhanced Deposition from Flow Development on Support}

As illustrated in Figure 9.4, deposition is enhanced over entrance lengths because the boundary layer in the developing flow has not reached its full thickness. [Note that the mechanism of enhanced deposition over the flow-development length was deduced from heat transfer analogies, and not from flow visualization tests, and was therefore not included in Figure 6.4.] Flow development is accounted for in TSFOUL in the expressions for particle diffusion and inertial coasting (Equations 8.39, 8.40 and 8.52), and is considered only for the support wall beyond the vena contracta and the point of 
re-attachment of the boundary layer. The other flow regions are either fully-developed (e.g., free-span region and along the tube surface in the support region ${ }^{12}$ ), or have a separated boundary layer where diffusion or coasting toward the wall is hampered (vena contracta region). Thus,

Separated boundary laver: For support only, in vena contracta region : $K_{D}=0$ and $K_{I}=0$ on support.

Entrance region: For support only, between $z_{a r t}$ and end of support : for $K_{D}$ use Equation 8.39 (turbulent) or Equation 8.40 (laminar), for $K_{l}$ use Equation 8.52. The first calculation of $K_{D}$ or $K_{1}$ beyond the re-attachment location $z_{a t t}$ is at the end of the first node, at $z=1 \mathrm{~mm}$ (as infinite $K$ values would result with $z=0$ ).

Fully-developed: For all other surfaces, use the equations with $z=1000 \mathrm{~mm}$ (based on supports being $\sim 1 \mathrm{~m}$ apart), which is equivalent to using the fullydeveloped versions of the equations.

Entrance effects would contribute in a minor way to the formation of the characteristic lip of deposit at the support inlet. In practice, however, deposition by diffusion and inertial coasting may be swamped by other mechanisms, so the entrance effect may not be noticeable. The modeling of this phenomenon would benefit from a more appropriate entrance-effect expression for $\mathrm{K}_{\mathrm{l}}$, and from better estimates of re-attachment locations.

\subsection{Gravitational or Centrifugal Settling on Top of Support}

Flow patterns observed during experiments showed that the region downstream of the support typically features a large recirculation or stagnation zone directly above the support (see Figures 5.4a to 5.4c). Gravitational settling would occur when flow is near-

\footnotetext{
${ }^{12}$ There will be some perturbation (thinning) of the boundary layer on the tube surface when the flow accelerates as it enters the support, and hence some increase in mass transfer to the tube. The effect is, however, small compared to that of a newly-developed boundary layer.
} 
stagnant above the support. The deposition coefficient $\mathrm{K}_{\mathrm{G}}$ is based on Stokes' Law, and is expressed as in the SLUDGE code (see Equation A.18):

$$
K_{G}=\frac{\left(\rho_{s}-\rho_{f}\right) g D_{s}^{2}}{18 \rho_{f} v_{f}}
$$

Centrifugal settling is similar to gravitational settling, except that the particles are under the influence of centrifugal and not gravitational acceleration. Centrifugal settling would occur when flow is recirculating directly above the support. The deposition coefficient $\mathrm{K}_{\mathrm{C}}$ is expressed as in Equation 9.8:

$$
K_{C}=\frac{\left(\rho_{s}-\rho_{f}\right) u_{f \text { sop }}^{2} k_{c . t o p} D_{s}^{2}}{18 \rho_{f} v_{f}}
$$

The streamline curvature $k_{c, 10 p}$ is the inverse of the radius $\left(k_{c}=1 / R\right)$, which is taken as $\sim 1 / 2$ the width of the horizontal surface based on flow observation. The local velocity $u_{\text {f.top }}$ is taken as $\sim 1 / 10$ that of the mainstream, based on experimental measurement of recirculation velocities (see Figure 4.12). A sketch of the settling processes is shown in Figure 9.5.

As outlined in Section 9.2, not all particles in the flow are subject to involvement in the settling process; this is described approximately by:

$$
K_{G \text { orC }}(n e t)=K_{G o r C}\left(1-N_{\text {smp }}\right)\left(1-\beta_{\text {sup }}\right) N_{s t}
$$

Here, instead of the flow area ratio term $C_{c}$ used in Equation 9.10, the support porosity $\beta_{\text {sup }}$ is used. $\beta_{\text {sup }}$ represents the ratio of support flow area to free-span flow area; $\left(1-\beta_{\text {sup }}\right)$ is therefore the ratio of support cross-sectional area to free-span flow area, and is taken to 
(approximately) equal the fraction of the particles that would be subject to deposition onto the top surface of support.

Because sticking is already accounted for, $K_{G}$ and $K_{C}$ are not subject to limitation by $K_{A}$, and are therefore treated independently from the classic transport-attachment model. [Another way of viewing this is to consider that gravitational or centrifugal forces downward toward the horizontal surface would help to overcome particle-surface repulsive forces.] For the downstream horizontal surface of the support (Node 46), the overall deposition coefficient is therefore simply:

$$
K_{\text {i .sup }}(i=46)=\left(K_{G} \text { or } K_{C}\right)
$$

Visual observations are used to determine whether the settling on the downstream surface is gravitational or centrifugal. In flow visualization experiments using boiling Freon-1 1 as a two-phase fluid (see Section 5.2), recirculation eddies were observed downstream of the support under conditions of low void and high velocity, and for supports with thick bars (support porosity $\beta_{\text {sup }} \approx<0.6$ ). Under these conditions, centrifugal settling would be the deposition mechanism. At higher void fractions $\left(\geq 30 \%\right.$, corresponding to $\chi_{\mathrm{th}} \geq 0.02$ in the SG) and for thin-barred supports ( $\beta_{\text {sup }} \geq 0.6$ ), the recirculating bubbly flow turned to stable vapour pockets, so the deposition behaviour is then better described by gravitational settling. Gravitation also best describes any very low-velocity situation, specifically when the mainstream velocity $u_{f}$ is less than $10 \sqrt{ }\left(g / k_{c}\right)$, at which point $K_{C}=$ $K_{G}$. In summary,

If $\chi_{\text {Ih }}<0.02$ and $u_{f}>10 \sqrt{ }\left(g / k_{c}\right)$ and $\beta_{\text {sup }}<0.6$, then use $K_{c}$, otherwise use $\mathrm{K}_{\mathrm{G}}$.

Gravitational and/or centrifugal settling appear to be dominant mechanisms in the upper regions of the SG on the top of the support, where deposits tend to be thin and smooth. 
The modeling of these phenomenon would benefit from a better expression for the fraction of particulates in the flow that become trapped within the region directly above the support.

\subsection{Modeling Parallel Channels in Support}

TSFOUL models two adjacent flow channels in the support, called the $Y$ and $Z$ channels. These channels can have different sizes (i.e., channel widths). As illustrated in Figure 9.6, maintaining the same pressure drop across both channels requires that the smaller $(Z)$ channel have a lower flow rate, because friction loss is greater in a smaller diameter channel, all else being equal (see Equation 7.17). As the flow rate affects many deposition parameters, the deposition behaviour in such a parallel arrangement is not straightforward-it is not intuitive which channel will foul more rapidly. TSFOUL is a useful tool for exploring these conditions.

Before TSFOUL calculates deposition coefficients for the nodes in the support region, it establishes the flow rate and flow conditions (e.g., void fractions, Reynolds numbers, friction factors, two-phase multipliers) in each channel iteratively:

1. Guess flow distribution based on area ratio between $Y$ and $Z$.

2. Calculate flow conditions and pressure losses in each channel.

3. Check if $\Delta P_{\text {tot }}(Y) \approx \Delta P_{\text {tot }}(Z)$; if not, adjust channel flow rates based on magnitude of discrepancy and repeat Step 2.

4. Flow rates are established; calculate deposition parameters.

5. Update channel flow areas based on calculated deposit thicknesses ( $\rightarrow$ feedback effect).

6. For next time interval, repeat Steps 1 to 5. 
This model may help to explain why, during SG inspections, severely blocked support holes have been found next to open ones.

The next Chapter describes predictions made using the TSFOUL program, in particular those involving deposition models for the support.

\section{References}

[1] W.E. Ranz and J.B. Wong, "Impaction of Dust and Smoke Particles on Surface and Body Collectors", Industrial and Engineering Chemistry, Vol.44 (1952 June), pp.1371-1381.

[2] J.W. Cleaver and B. Yates, "A Sub-Layer Model for the Deposition of Particles from a Turbulent Flow", Chemical Engineering Science, Vol.30, 1975, pp.983992.

[3] D. Hasson, A. Marmur, and Y. Tor, "Fouling of a Cylindrical Obstacle Placed in an Air Stream", Proceedings of the 7th Int. Heat Transfer Conference, Vol. 6, p.301 (1982).

[4] N. Epstein, "Particulate Fouling of Heat Transfer Surfaces: Mechanisms and Models", Proceedings of the NATO Advanced Study Institute on Advances in Fouling Science and Technology, Alvor, Algarve, Portugal, 1987 May 18-30, also printed as NATO ASI Series E: Applied Sciences - Vol.45 by Kluwer Academic Publishers, 1988. 
[5] C.W. Turner, M.E. Blimkie, and P.A. Lavoie, "Physical and Chemical Factors Affecting Sludge Consolidation", Report No. COG-96-492-I, AECL-1 1674, 1997 September. 
Figure 9.1: Sketch of particle impaction on support bottom

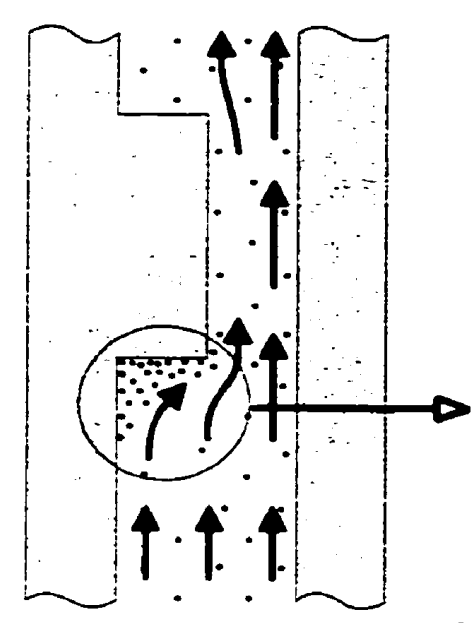

impaction of particles some stick to surface

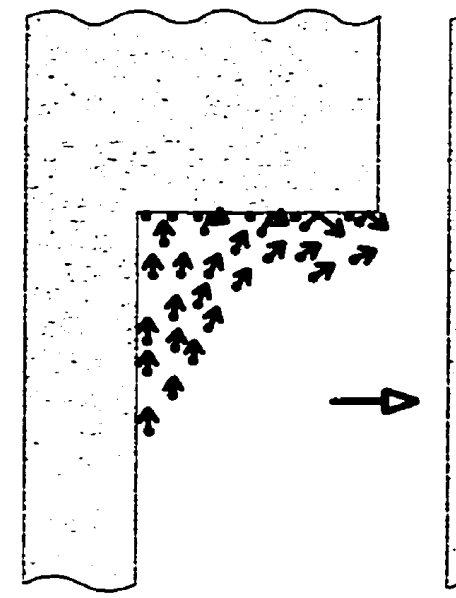

dynamic forces help to pin particles to surfaces 
Figure 9.2: Sketch of flashing and consolidation phenomenon

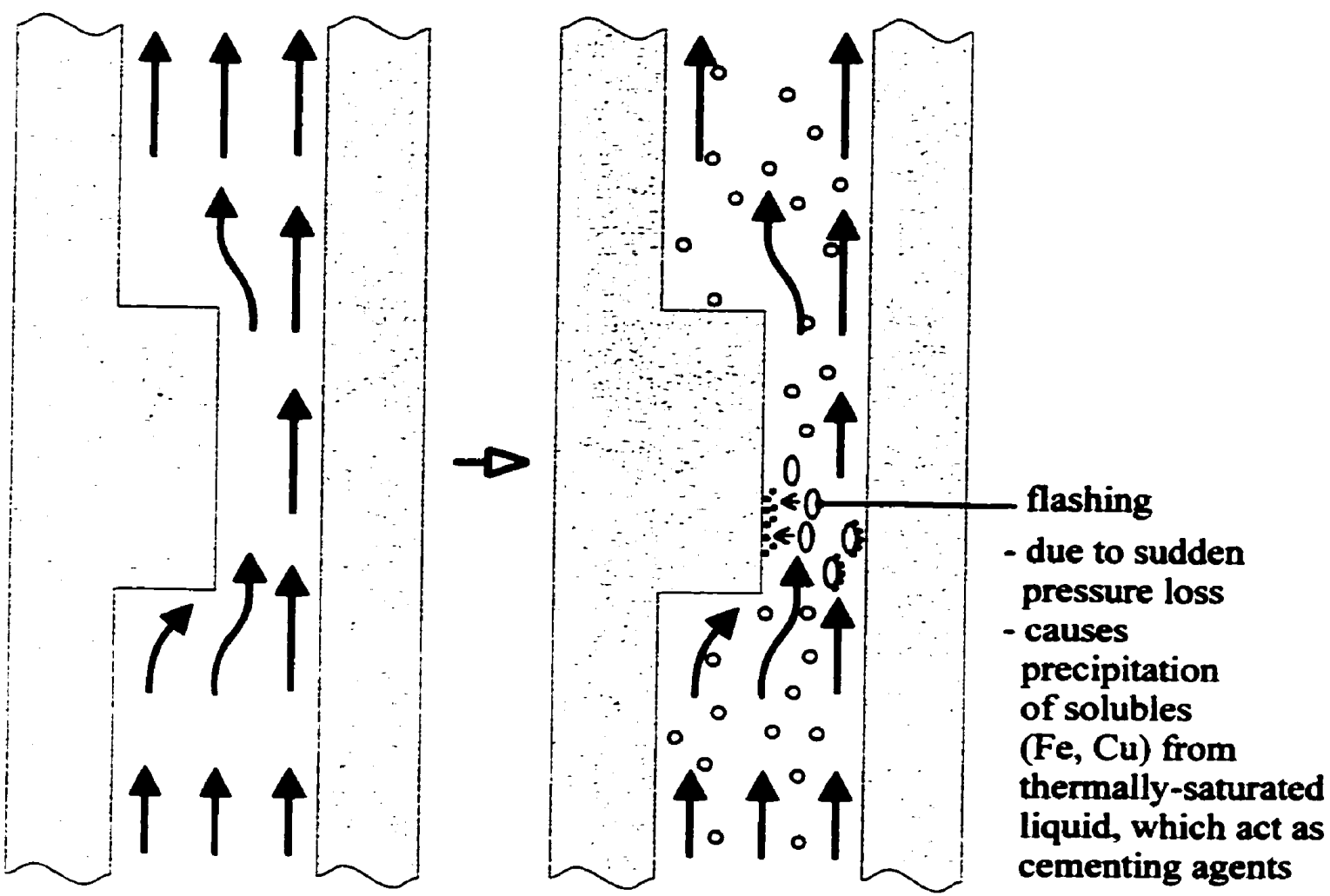


Figure 9.3: Suppression factor $A$ as a function of precipitate fraction

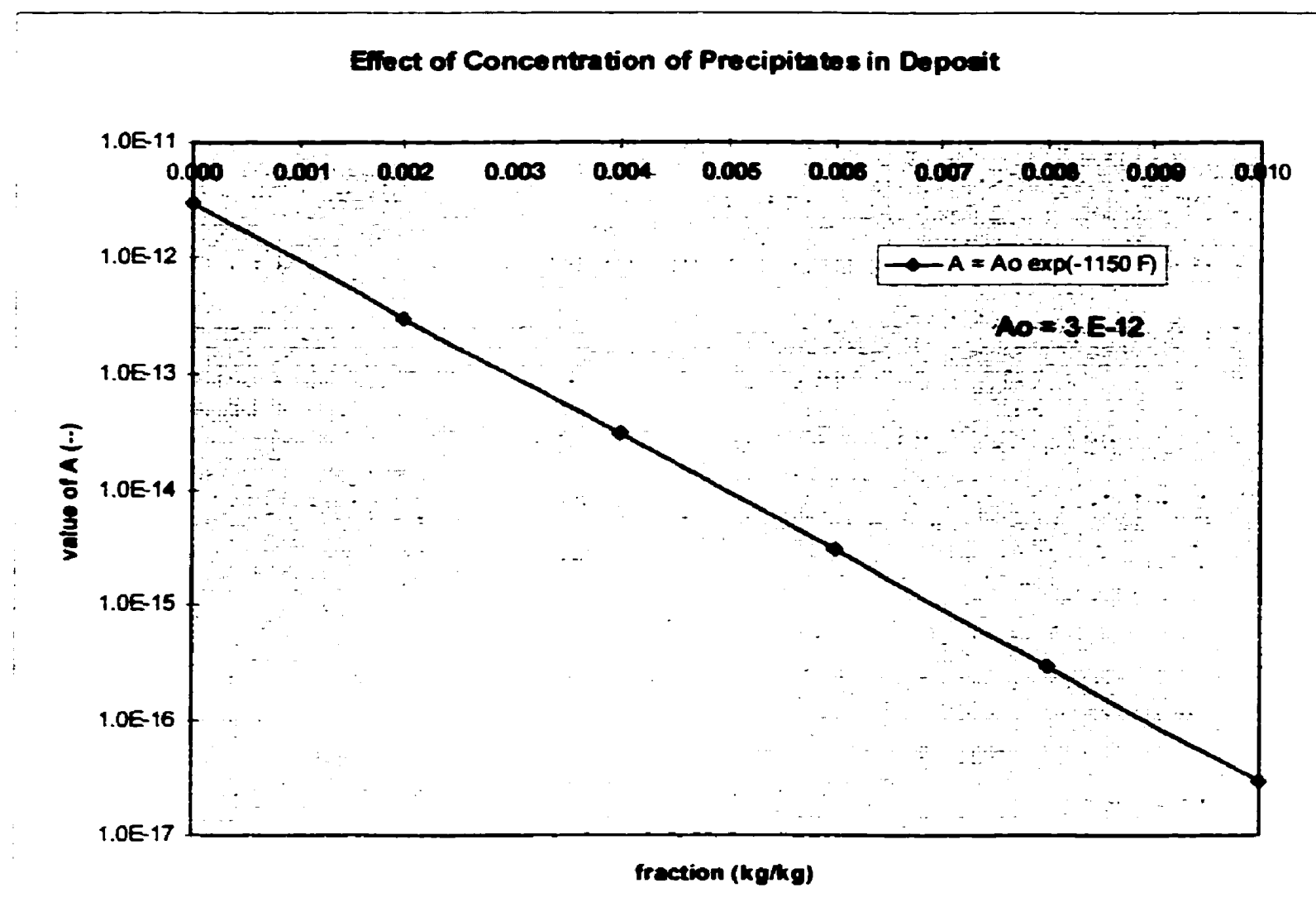


Figure 9.4: Sketch of enhanced deposition in developing regions

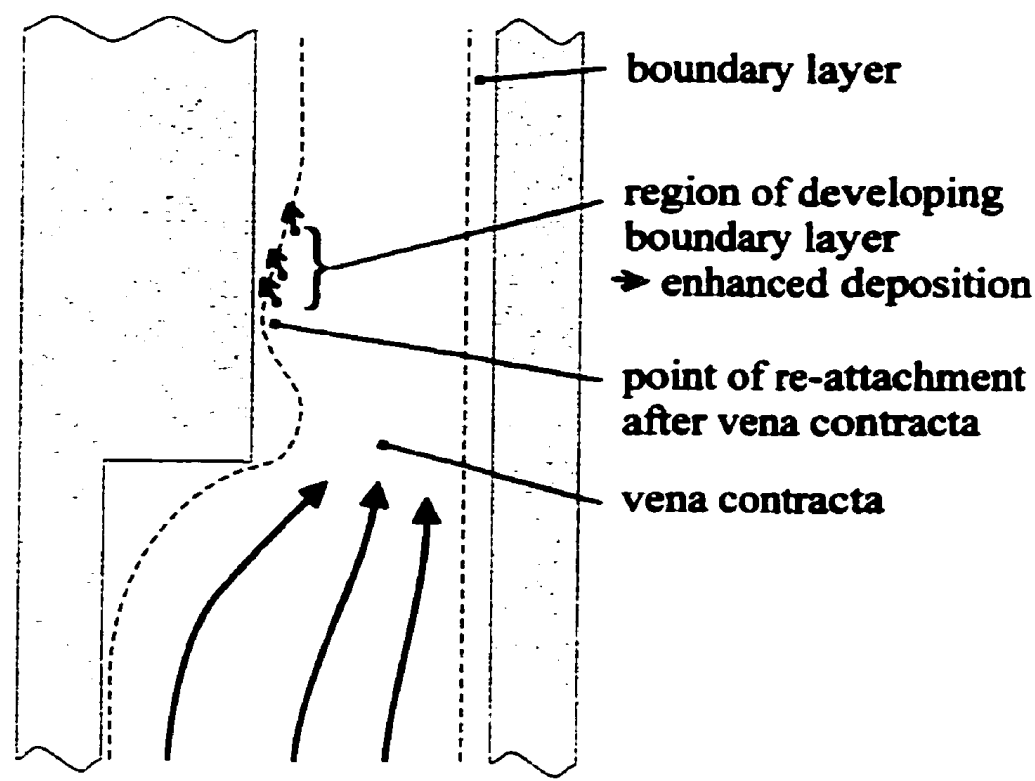


Figure 9.5: Sketch of settling on top of support

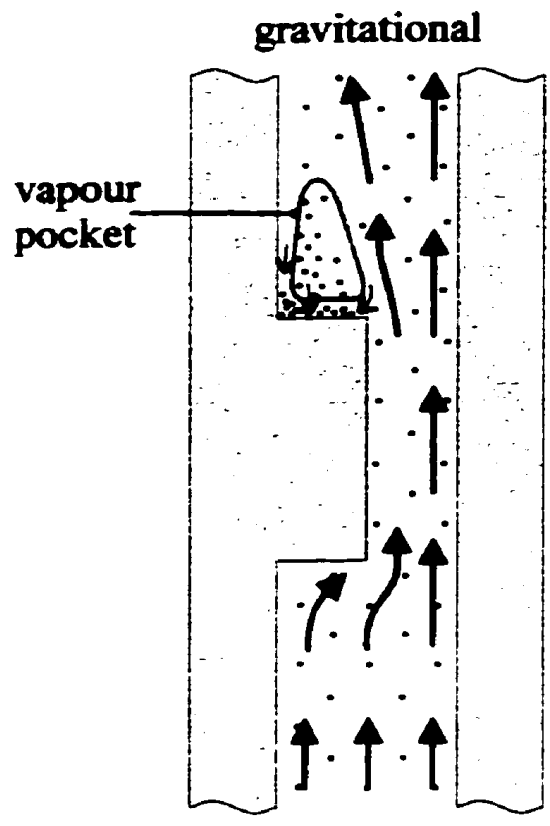

higher quality centrifugal

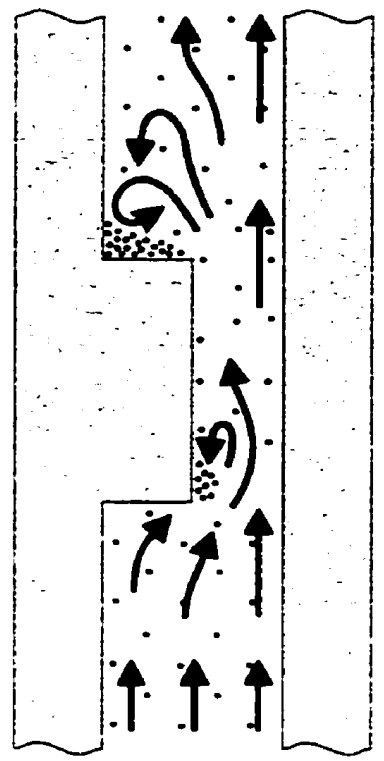

lower quality, higher velocity, lower support porosity 
Figure 9.6: Sketch of unequally-sized channels in support

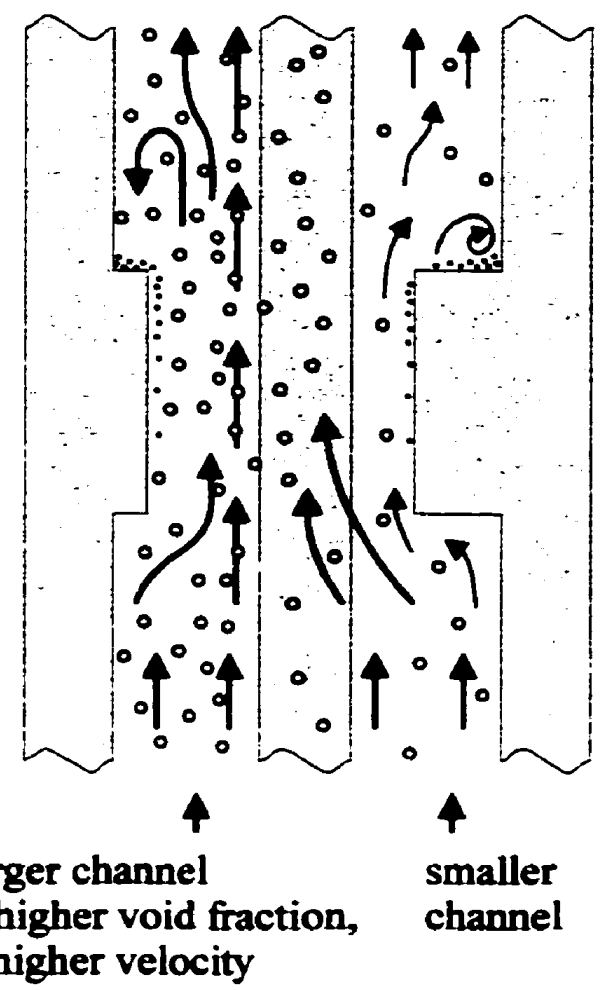




\section{Chapter 10}

\section{PREDICTIONS OF DEPOSITION}

This chapter describes predicted deposition profiles and rates using the TSFOUL program. Section 10.1 covers only the classic deposition models described in Chapter 8 (thermophoresis, inertial coasting, diffusion, attachment, and boiling). Section 10.2 covers both the classic models described in Chapter 8 and the support-specific models described in Chapter 9 (impaction, centrifugal and gravitational settling, consolidation, flow-development effects, and parallel-channel effects). A description of the flags used to turn certain models on and off are listed in Table 10.1.

This chapter also presents, in Section 10.3, two validation exercises to determine the accuracy of the fouling models. A comparison was made with deposit thicknesses from short-term support fouling experiments, as well as with observed long-term fouling trends at the Bruce A SG. Section 10.4 summarizes the main results from the predictions.

\subsection{Classic Deposition Models}

Figures $10.1 \mathrm{a}$ and $10.1 \mathrm{~b}$ show predictions using only the classic models described in Chapter 8: thermophoresis, inertial coasting, diffusion, attachment, and boiling. The flags for support-specific models, FKF, FKV, FKS, FCO, FFD, and FCH (see Table 10.1) were set to zero. This prediction serves as a reference for later comparison with results using support-specific models. The input values, listed in Table 10.2, are typical values for the Bruce A SG. A schematic of the idealized geometry is shown in Figure 7.1.

Figure 10.1 a shows the predicted deposit profiles on the tube and support surfaces, as a function of distance, after a time period of 1 year. A comparison between the two profiles 
shows that the tube surface has thicker deposits than the support, owing to the boiling term which is only in effect on the heated tube surface. With regard to the deposit profile for the tube, the deposit is thinner within the support region (distance 21 to $45 \mathrm{~mm}$ ) than within the free-span region, because the increased velocity enhances the deposit "removal" or suppression mechanism. ${ }^{13}$ [This reference case ignores any accumulation in the vena contracta eddy, which is described in Section 10.2.3.] However, variations within the support region show the opposite trend: a thicker deposit is predicted for the support inlet (i.e., the vena contracta region, distance 21 to $36 \mathrm{~mm}$ ), which has a higher velocity than the support outlet. The reason for this opposite trend is that, within the support region, attachment is not limiting due to the high kinetic energy, and transport therefore dictates the deposition coefficient; the transport term (which gives increasing deposition with velocity) wins over suppression (which gives decreasing deposition as velocity increases). This is a good example of how mechanisms compete on even the simplest parallel surfaces.

Figure $10.1 \mathrm{~b}$ illustrates the growth of the deposit on the tube in the free-span region and the support region, as a function of time. It shows that, in turbulent flow, the deposit does not grow linearly but approaches a constant thickness over time. Again, this is linked to the suppression model which has an exponential term in turbulent flow (see Equation 8.1 and the associated discussion on reasons for asymptotic behaviour). The deposit within the support region approaches its asymptote more quickly because of the higher velocity and hence stronger suppression term.

Table 10.3 lists the deposition coefficient (or $\mathrm{K}$ ) terms for the reference case after a time period of one year, to show the relative magnitudes. The last row in this table shows the overall coefficients that were used in Equation 8.1 together with the suppression term to calculate deposit thicknesses. Table 10.3 shows that thermophoresis draws particles away

13 This behaviour is consistent with the expectation during the design of broached-plate supports that the increased velocity would "scour" the broach holes and discourage deposition, which first raised the question of how SG supports could possibly become blocked. 
from the heated tube (as shown by negative sign), but is a very weak contributor, as expected; it is zero for the support which is an adiabatic surface. Contributions by other mechanisms vary depending on whether particles are in the free-span region or in the support region. In the free-span region (Nodes 1 and 65 in Table 10.3), diffusion and inertial coasting are of similar magnitude; transport is limited by a weak attachment term. but the boiling term swamps all terms and dictates the overall coefficient $K_{\text {overall. In the }}$ support region (Node 21), the attachment term is very large due to high kinetic energy, and deposition is now limited by the transport term; however, due to the higher velocities, the transport term itself is significant (with inertial coasting dominating over diffusion), in fact it is larger than the boiling term. The $\mathrm{K}_{\text {overall }}$ values for the support region are quite large and would lead to very thick deposits (growing at a rate of $\sim 40 \mu \mathrm{m}$ per month), if it weren't for the suppression term.

Figures $10.2 \mathrm{a}$ to $10.2 \mathrm{e}$ give the results of sensitivity runs, in which the following input parameters were varied: mass flux $G$, steam quality $\chi$, particle concentration $\phi$, particle diameter $D_{s}$, and suppression factor $A$. These are for the tube surface in the free-span region, simply to show trends. [The trends would be similar for the support region, except that they would be less pronounced because of the greater suppression effect there.] Deposit thickness decreases as $G, \chi$, or $A$ increases-a direct result of the suppression effect, in which faster-moving fluid suppresses deposition more, higher quality promotes faster liquid flow, and a higher suppression factor limits growth. Conversely, deposit thickness increases as $\phi$ or $D_{s}$ increases-the more particles or the bigger the particles, the more deposit.

An interesting result shown in Figure $10.2 b$ (variation with quality $\chi$ ), is that very little deposition is predicted when no boiling takes place. This illustrates the impact of the boiling term $\mathrm{K}_{\mathrm{B}}$. Another interesting feature is, in Figure 10.2d (variation with $\mathrm{D}_{\mathrm{s}}$ ), the insensitivity to particle size between 1 and $0.1 \mu \mathrm{m}$. This is because the reduced build-up of deposit (smaller particles give thinner layers) is offset by an enhanced diffusion rate (diffusivity is greater with smaller particles). 


\subsection{Support-Specific Models}

10.2.1 Effect of flow regime: turbulent vs laminar

Figure 10.3 shows how the rate of deposit growth can be significantly greater when laminar flow is accounted for. For these runs, the inlet mass flux was chosen to give a Reynolds number just below the transition value of 3000 . With laminar flow (FLM $=1$ ), no suppression is applied, according to Equation 8.2. If the flow is assumed to be turbulent $(\mathrm{FLM}=0)$, deposit growth is subject to suppression.

Note that, at these very low mass fluxes, the suppression factor A needed to be strong to show any regime effect (e.g., with the original SLUDGE value of $7 \times 10^{-11}$ instead of the reference value of $3 \times 10^{-12}$ ). Otherwise, the low velocity together with a weak suppression factor in turbulent flow made very little difference when compared with the laminar (no suppression) result. Because a suppression factor of $7 \times 10^{-11}$ is considered unrealistic, this Figure serves only to illustrate the flow regime effect. [A more realistic situation that shows the effect of flow regime is that encountered in unequally-sized parallel channels, described in Section 10.2.6.]

The two traces on Figure 10.3 curve slightly upwards, which is opposite to the usual asymptotic trend. This is due to a slightly increasing mass flux over time which, in tum, is due to an unusual deposition pattern. As particle transport is reduced under these very low flow conditions, the main contributor to deposition becomes boiling, which is flowindependent. Due to greater suppression in the support region (compared with the freespan region), the flow area ratio between free-span and support actually decreases, leading to decreasing flow resistance from sudden contraction and expansion. 


\subsubsection{Effect of particle impaction on support bottom}

Figure 10.4a shows the effect of particle impaction on the bottom horizontal surface of the support (at Node 20). For this prediction, a large particle size $(8.5 \mu \mathrm{m})$ was required, as the reference size of $\mathrm{l} \mu \mathrm{m}$ gave insufficient momentum for impaction and hence no deposition. [A lower particle concentration of $0.05 \mathrm{ppm}$ was chosen instead of the reference value of $0.5 \mathrm{ppm}$, to somewhat compensate for the larger particle size.] Over time, the deposit grows until its maximum size of half the surface width is reached, as per the criterion discussed in Section 9.1. Note that the deposit thickness on the horizontal surface is predicted to be in the $\mathrm{mm}$ range rather than the $\mu \mathrm{m}$ range. This order of magnitude is in agreement with observations in the SG (see Section 2.1.5) and in flow visualization experiments (Section 4.3.3). This implies that the use of only $1 \mu \mathrm{m}$ as reference particle size is too limiting, as it does not account for deposits on the bottom of SG supports.

[The reference value of $1 \mu \mathrm{m}$ was initially chosen based on the observed fine deposits on the Bruce A SG supports. In general, on supports there is a mix of colloidal particles and precipitated solubles. However, magnetite particles circulating with the SG flow can range from 0.5 to $20-30 \mu \mathrm{m}$ in size, and particles can agglomerate to form larger clusters. This further underscores the need to account for a range of particle sizes in the modeling.]

For the same conditions and location (Node 20), Figure 10.4b shows the deposit thickness as a function of particle size and mass flux, at an elapsed time of 2 months. The particle impaction mechanism is clearly very sensitive to particle diameter. Figure $10.4 \mathrm{~b}$ indicates that, for a given set of conditions, there is a narrow range of particle diameter below which an insignificant deposit is created and above which the deposit is large after only a short time. This is a direct result of the criteria of Equation 9.3 in the particle impaction model. 
According to Figure $10.4 \mathrm{~b}$, for a mass flux of $300 \mathrm{~kg} / \mathrm{m}^{2} . \mathrm{s}$ (reference condition). particles do not impact and stick unless they are greater than 8 microns. For the higher mass flux of $500 \mathrm{~kg} / \mathrm{m}^{2} . \mathrm{s}$, the threshold is lower ( 6 microns), because a given particle then arrives at a greater velocity and will impact the surface even if it is smaller.

As with the previous figure, the results were obtained with a lower particle concentration of $0.05 \mathrm{ppm}$. Again, this was meant to account for (in a crude way) the fact that larger particles are generally present in the $S G$ at lower concentrations than smaller particles. The deposit would probably not grow as quickly as Figure $10.4 \mathrm{~b}$ suggests (where the deposit for the larger particle sizes reaches its maximum size in only two months). In reality, there is a spectrum of concentrations just as there is a diversity of particle sizes.

\subsubsection{Effect of centrifugal and gravitational settling}

Figure 10.5a is an axial deposit profile along the tube and the support after a time period of 1 year, showing the effect of centrifugal settling in the vena contracta region, and (in this case, gravitational) settling on the top surface of the support. These profiles are the same as those in Figure 10.1 a except for the settling on the support. This figure clearly shows the sine-shaped deposit on the support beyond the entrance (20 to $36 \mathrm{~m}$ distance), as per Equation 9.9, and a deposit on top of the support from gravitational settling (at 46 mm distance) according to Equation 9.18.

Figure 10.5b shows the effect of particle size on the deposit thickness at the throat of the vena contracta itself (Node 28 , at a distance of $8 \mathrm{~mm}$ from the support inlet), and on top of the support (Node 46), after a time period of 1 year. Not shown on this graph is an associated reduction in mass flux: as the deposit thicknesses at various locations in the support region increase with particle diameter, the (free-span) mass flux decreases to retain the same overall pressure loss $(-15 \mathrm{kPa})$ over time. This mass flux starts at $\mathrm{G}=300$ 
$\mathrm{kg} / \mathrm{m}^{2} . \mathrm{s}$ for the thin deposits from small particles, but is reduced to $225 \mathrm{~kg} / \mathrm{m}^{2}$.s by the time the particles are $4 \mu \mathrm{m}$ in diameter.

For both curves in Figure 10.5b, as particle size increases, rapid deposition (from settling being a function of $D_{s}$ squared) is offset by two factors: (1) decreased deposition from reduced mass flux and hence particle transport, and (2) decreased deposition from larger particles having sufficient inertia to travel straight and not get entrained backward into the eddy. However, the deposition due to gravitational settling on the top is not significantly suppressed because the local velocity is low, and the deposit becomes very thick with large particles (and with time). [In practice, particle sizes greater than $1 \mu \mathrm{m}$ would be present at concentrations lower than $0.5 \mathrm{ppm}$, so the deposit would not grow so extensively so quickly.] The deposition due to centrifugal settling in the vena contracta region is much more suppressed than beyond the support because the mass flux is higher in the narrowed channel.

One important difference between settling on top of the support and settling in the vena contracta region is that the latter is ultimately self-limiting--sustained deposition within the flow channel would eventually choke the channel and drastically reduce the mass flux and hence the supply of particles. This is illustrated in the predictions for parallel channels, described in Section 10.2.6.

\subsubsection{Effect of flashing and consolidation}

Figure 10.6a shows the variation of the suppression factor $A$ with distance, following the model outlined in Section 9.3, after a time period of 1 year. The model allows a reduction of $A$ as a function of the quantity of precipitate, which is a function itself of quantity of liquid vaporized due to flashing, which is a function of pressure reduction. Note that, to obtain even the slightest variation in $A$, the concentration of solubles had to be increased by a factor of 200 from the reference (and more realistic) value of $5 \mathrm{ppb}$ (as 
per Table 10.2). This indicates that for the model to have any effect, a considerable pressure loss is required (e.g., $>12 \mathrm{kPa}$ which is the loss at the inlet, between 20 and 21 mm distance in Figure 10.6b).

Figure $10.6 \mathrm{~b}$ shows the calculated pressure profile under the same conditions. The overall pressure loss of $\sim 15 \mathrm{kPa}$ remains fixed over time (but the shape of this pressure profile can change as the deposits grow). There is a relatively large pressure loss at the support inlet - but small compared to the absolute pressure of 4.4 MPa - which determines the extent to which the A value (Figure 10.6a) is reduced. Precipitates from the inlet are assumed to be spread over the vena contracta region, so $A$ is made to be low over this entire region.

The corresponding deposit profiles for the time period of 1 year are shown in Figure 10.6c. To better show the effect of $A$ reduction, the deposit profile obtained in Figure 10.5a with the settling models turned on was used as comparison. The reduction of $A$ in the vena contracta region has allowed the deposit there to grow slightly thicker on both support surface and the tube surface. The effect becomes more pronounced as the flow area ratio is reduced and the pressure loss is higher (not shown here); however, competition arises as the channel velocity is then increased. As $A$ and $u^{*}$ are both in the suppression term for turbulent flow, and can cancel each other out. In fact, $\mathbf{u}^{*}$ tends to dominate in the suppression term, which severely limits the effectiveness of this consolidation model.

\subsubsection{Effect of developing flow}

Figure 10.7 shows the deposit profile over distance, after a time period of 1 year, for the reference case (classic models only) and for the flow-development and settling models on $(F F D=1, F K V=1, F K S=1$ ). Comparison with only the settling models on (FKV=1, FKS $=1$, shown in Figure 10.5a) shows that only the region beyond the vena contracta, 
i.e., beyond the point of boundary layer re-attachment (distance 37 to $45 \mathrm{~mm}$ ), is affected by the flow development. As expected, the deposit in that region is calculated to be slightly thicker when flow development is considered than when it is neglected. During flow development and growth of the boundary layer, deposition by diffusion and inertial coasting (and hence the transport term) starts at a maximum and is gradually reduced. The enhancement in deposition from the accounting for flow development is quite modest.

\subsubsection{Effect of unequally-sized parallel channels}

Figures $10.8 \mathrm{a}$ to $10.8 \mathrm{c}$ show the effect of unequally-sized parallel channels in the support, after a time period of 1 year. For all figures, the $Y$ channel width was kept constant while the $\mathrm{Z}$ channel width was decreased. Because the total flow area in the support region therefore decreased as well, the mass fluxes in the channels varied from one geometry (i.e., channel width ratio) to the next. Also, for a particular geometry, the initial mass flux was allowed to vary over time to keep the overall pressure loss fixed.

Figure 10.8a shows the effect on the void distribution between the larger $Y$ channel and smaller $Z$ channel, for two initial free-span mass fluxes ( $G=80$ or $\left.300 \mathrm{~kg} / \mathrm{m}^{2} . s\right)$. The void fraction in $\mathrm{Z}$ is always less than that of the more open $\mathrm{Y}$ channel. This is due to channel mass fluxes and void fractions having to adjust to maintain a constant pressure loss across the support. To compensate for the higher flow resistance from friction and obstruction in the smailer $Z$ channel, the void fraction (upon which the two-phase multiplier is indirectly dependent) must decrease. [This occurs whether there is boiling on the tube surface or not.] Note that a large difference in subchannel sizes is required before the void distribution is affected significantly.

Figures $10.8 \mathrm{~b}$ and $10.8 \mathrm{c}$ show the effect on the deposit thicknesses on the tube within the two channels, for the two initial mass fluxes, respectively. There are multiple effects originating from the unequal sizes; these can be explained in moving from right to left in 
these figures. At the reference mass flux of $300 \mathrm{~kg} / \mathrm{m}^{2} . \mathrm{s}$ (Figure $10.8 \mathrm{~b}$ ), the deposits are thicker when the width ratio (i.e., $Z$ channel width) is less, due to lower mass fluxes in the support channels and hence less suppression. At some point (below width ratio of $\sim 0.4$ ), the deposition in the $\mathrm{Z}$ channel peaks. Further reduction in $\mathrm{Z}$ width does not increase the $\mathrm{Z}$ deposit thickness further (in fact, decreases it), because the increasing flow resistance then begins to limit the mass flux in the $Z$ channel, and hence the particle transport to that channel. When $Z$ is very narrow, TSFOUL has difficulties converging on the flowdistribution iteration. For the result at the smallest width ratio, the flow regime is still turbulent, with a $\operatorname{Re}$ value of $\sim 5000$ within the $Z$ channel.

A similar trend occurs at the initial mass flux of $80 \mathrm{~kg} / \mathrm{m}^{2} . s$ (Figure 10.8c). The deposits are thinner than at the higher mass flux (previous figure), due to less mass transport overall. At a width ratio of 0.2 , the flow in the $Z$ channel has crossed the regime threshold to laminar flow, resulting in no suppression and a significantly thicker deposit. As $Z$ is reduced further to give a width ratio of 0.15 , opposing deposits on the tube and support surfaces are predicted to be thick enough after $I$ year to block the $Z$ channel.

The reference-case results (Figure $10.8 \mathrm{~b}$ ) suggest that deposition in a much smaller parallel channel would be limited at some point by the reduced availability of particles. [One can speculate that, especially for large particles, other mechanisms such as impaction and settling may then become the dominant mechanisms for deposition near this channel, and may eventually cause complete channel blockage.] The lower mass-flux case (Figure 10.8c) suggests that, if the overall flow rate is low enough that the smaller channel experiences transition to laminar flow, the deposit can grow rapidly in spite of the reduced particle transport, and cause blockage. 


\subsection{Validation of Models}

\subsubsection{Comparison with Short-Term Fouling Experiments}

A comparison was made between TSFOUL predictions and the results of the simple fouling experiment described in Section 2.1.6 and shown in Figure 2.3. This experiment involved a bifoil broached support subjected to a flow of magnetite suspension at high temperature and pressure (representative of SG conditions) for 600 continuous hours.

Figure 10.9 shows the calculated profile for deposit thickness, for both tube surface and support surfaces, with the new support-specific models in effect. Because there was no boiling on the tube in this experiment, minimal deposit is calculated for the tube surface. Because of the support-specific models, however, a small deposit is expected in the inlet (vena contracta) region of the support and on the top surface of the support. Particle impaction on the upstream face of the support is not expected as the magnetite particles were very small $(0.25 \mu \mathrm{m})$.

The calculated results can be compared with the actual deposit pattern, shown in Figure 2.3, which consists very little deposit in general, but a somewhat more extensive deposit at the support inlet. The amount of deposit on the top surface was not recorded at the time of the test. TSFOUL did not predict very thick deposits when none were observed; however, the thicknesses seem to be underpredicted even with the use of the supportspecific models. More information from fouling experiments is clearly needed to finetune models in TSFOUL for better quantitative agreement.

\subsubsection{Comparison with Long-Term Fouling at Bruce A SG}

Comparisons were made between TSFOUL predictions and the deposition observed in the Bruce A SG as described in Section 2.1.3 and shown in Figure 2.2. The simulations 
(Figures $10.10 \mathrm{a}$ to $10.10 \mathrm{e}$ ) were done with conditions and geometry representative of the Bruce SG, with all models in effect and for a time period of 10 years.

Figure 10.10a shows that predicted deposit thicknesses are quite modest - up to $\sim 30 \mu \mathrm{m}$, except on top of the support which wouldn't contribute directly to support blockage. The $1 \mu \mathrm{m}$ particles do not have enough momentum to deposit on the bottom of the support (at $20 \mathrm{~mm}$ distance), so no deposition is predicted there. A deposit would be expected in the vena contracta region on the support ( 21 to $36 \mathrm{~mm}$ ).

Figure $10.10 \mathrm{~b}$ is with the same conditions as the previous figure, except that the suppression factor $A$ is an order of magnitude lower, from $3 \times 10^{-12}$ to $3 \times 10^{-13}$. Note that this lower value is more than two orders of magnitude lower than the factor $7 \times 10^{-11}$ used in the original SLUDGE code (see Appendix A). Even with the reduced suppression (i.e.. with enhancement), the predicted deposit thickness is not excessive, only $0.25 \mathrm{~mm}$ in 10 years on the tube surface. Clearly, the ability to predict thicknesses realistically would depend heavily on determining the appropriate $A$ value, perhaps by experiments.

An attempt was made to predict deposition with zero suppression (i.e., vanishing A). It was found that very little further reduction in the suppression factor (to a value of $A=7 \times 10^{-14}$ ) was needed before deposits in the vena contracta region occupied most of the channel after less than 10 years. This is evident in Figure $10.10 \mathrm{c}$, which shows the growth of deposit on the tube in the vena contracta region for various values of $A$. [The prediction results for the $\mathrm{A}=7 \times 10^{-14}$ case are probably somewhat inaccurate as some models would break down when the channel geometry and conditions change radically.] At the 9.5-year mark with $A=7 \times 10^{-14}$, the mass-flux iteration (which adjusts the free-span mass flux based on maintaining a fixed pressure loss) could not converge. These convergence difficulties were likely due to local conditions changing rapidly from one time-step to the next. The flow-convergence problem is reminiscent of the flow oscillations experienced in the Bruce A SG when upper broached plates were severely fouled (see Section 1.2). 
Figures $10.10 \mathrm{~d}$ and $\mathrm{e}$ are with the same conditions as Figure 10.10a, except that the $\mathrm{Z}$ channel is made half the width of the $\mathrm{Y}$ channel. For a more realistic comparison with the reference case (Figure 10.10a) in the support region, the free-span mass flux was altered to give the same overall pressure loss of $15 \mathrm{kPa}$. [This gives comparable flow conditions within the channels, but not in the free-span region.] In the support region, the deposits thicknesses on the tube are similar for the two channels; there is a difference, however, in the deposits on the support surfaces. The smaller $Z$ channel features a lower velocity, and hence a shorter vena contracta region and less suppression; this makes its deposit there shorter and thicker.

Assuming the support fouling models in TSFOUL follow the correct trends, this result (thicker deposit on the support in $\mathrm{Z}$ ) and that of Figure 10.8a (thicker deposit on the tube in $Z$ ) suggest that a smaller channel in a parallel set experiences more rapid deposit growth. The models also suggest that the critical region for fouling and blockage would be the vena contracta region, on both the tube and the support surfaces.

\subsection{Simulation Uncertainty}

Particle deposition on SG tubes and supports is clearly a complex phenomenon involving many mechanisms that interact. While TSFOUL cannot predict the deposition behaviour with full accuracy, it has shed some light on these interactions (see following section for summary). The level of accuracy (or uncertainty) of the simulations is also difficult to determine, but the simulations are judged to provide better than order-of-magnitude agreement with actual deposition behaviour.

There appear to be two parameters that dominate the simulations described in this chapter, i.e., whose uncertainties dictate that of the entire simulation. One is the suppression factor $\mathbf{A}$, to which the predictions are very sensitive (as illustrated in Figure 10.10c). The other is the particle size, as some deposition mechanisms are in effect for 
only certain size ranges. More accurate values for these two parameters would greatly reduce the simulation uncertainty.

\subsection{Summary of Findings}

- In the free-span region, the dominant deposition mechanism is boiling (on the tube). In the support region, the transport mechanisms tend to dominate. However, in turbulent flow, a suppression effect based on a factor of $A=3 \times 10^{-12}$ generally supersedes both these effects.

- As expected, deposit thickness decreases as mass flux, quality, or suppression factor increases. The thickness increases as particle size or concentration increases.

- Particle impaction on the support bottom would occur under SG conditions if the particle size were relatively large (e.g., $>8 \mu \mathrm{m}$ ).

- Specifying a single particle diameter or concentration is limiting, as SGs carry particles of varying sizes and correspondingly varying concentrations.

- Gravitational and centrifugal settling appear to be sensible models for predicting build-up on the top of the support and at the vena contracta eddy.

- The consolidation model would be an meaningful way of accounting for enhanced deposition at the support inlet, except that its effect is dominated by the current suppression model.

- Accounting for developing flow increases the deposition on the support, but only modestly so.

- Among unequal parallel channels in the support, the narrower channel tends to have the lower void fraction and the greater deposit thicknesses.

- As the smaller channel (in an unequal parallel set) becomes narrower, its deposit thicknesses reach a limit beyond which no further growth occurs, due to the diminishing of the particle supply to that channel. If this channel experiences transition to laminar flow, the deposits are predicted to grow rapidly in spite of the reduced supply, possibly to the point of blockage. 
- A comparison with a laboratory fouling test shows that the deposition is underpredicted, even with the use of the support-specific models. More experimental data are clearly needed for model fine-tuning and validation.

- Long-term (10 year) predictions show modest deposition, except when the suppression factor $A$ is reduced. This shows that the suppression term is very dominant, and predictions rely heavily on establishing the appropriate $A$ value.

- At a value of $A=7 \times 10^{-14}$, deposits were predicted to occupy most of the support channel after $\sim 10$ years, at which point convergence difficulties occurred that were reminiscent of flow instability.

- The long-term predictions show that a narrower channel will eventually have a thicker deposit on its support than its wider neighbor.

- The critical region for fouling and blockage appears to be the vena contracta region, on both the tube and the support surfaces.

- The overall uncertainty of the simulations is dictated by that of the suppression factor A and that of the particle size assumption, and is difficult to establish. However, the simulations are judged to provide better than order-of-magnitude agreement.

The next Chapter summarizes the work, and offers recommendations on follow-up. 
Table 10.1: Model options in TSFOUL

\begin{tabular}{|c|c|c|}
\hline FLAG & VALUE & EXPLANATION \\
\hline FBO & $\begin{array}{l}=0 \\
=1\end{array}$ & $\begin{array}{l}\text { Boiling } \\
=\text { adiabatic flow; no boiling on SG tube } \\
=\text { diabatic flow; boiling on SG tube based on input heat flux }\end{array}$ \\
\hline FGK & $\begin{array}{l}=0 \\
=1\end{array}$ & $\begin{array}{l}\text { Inlet Mass Flux } \\
=\text { constant mass flux over time, based on input value } \\
=\text { inlet mass flux decreases with time as resistance increases }\end{array}$ \\
\hline FPR & $\begin{array}{l}=0 \\
=1\end{array}$ & $\begin{array}{l}\text { Phvsical Properties } \\
=\text { properties calculated at inlet and assumed constant } \\
=\text { properties vary as pressure changes axially }\end{array}$ \\
\hline FLM & $\begin{array}{l}=0 \\
=1\end{array}$ & $\begin{array}{l}\text { Laminar Flow } \\
=\text { assume flow is always turbulent } \\
=\text { if } \operatorname{Re}<3000 \text { then no "removal" and different } K_{D} \text {. }\end{array}$ \\
\hline FKF & $\begin{array}{l}=0 \\
=1\end{array}$ & $\begin{array}{l}\text { Particle Impaction } \\
=\text { no particle impaction on bottom of support } \\
=\text { particle impaction enabled }\end{array}$ \\
\hline FKV & $\begin{array}{l}=0 \\
=1\end{array}$ & $\begin{array}{l}\text { Settling in Vena Contracta Region } \\
=\text { no centrifugal settling in vena contracta region on support } \\
=\text { centrifugal settling enabled }\end{array}$ \\
\hline FKS & $\begin{array}{l}=0 \\
=1\end{array}$ & $\begin{array}{l}\text { Settling on Top of Support } \\
=\text { no gravitational or centrifugal settling on top of support } \\
=\text { gravitational and centrifugal settling enabled }\end{array}$ \\
\hline $\mathrm{FCO}$ & $\begin{array}{l}=0 \\
=1\end{array}$ & $\begin{array}{l}\text { Deposit Consolidation } \\
=\text { suppression factor A stays at initial input value } \\
=\text { A varies with quantity of precipitate from flashing }\end{array}$ \\
\hline FFD & $\begin{array}{l}=0 \\
=1\end{array}$ & $\begin{array}{l}\text { Flow Development } \\
=K_{D} \text { and } K_{D} \text { are for fully-developed flow } \\
=\text { entrance length is accounted for in } K_{D} \text { and } K_{I}\end{array}$ \\
\hline $\mathrm{FCH}$ & $\begin{array}{l}=0 \\
=1\end{array}$ & $\begin{array}{l}\text { Unequally-Sized Channels } \\
=Z \text { channel is made the same size as } Y \text { channel } \\
=Y \text { and } Z \text { channels have different sizes, as input }\end{array}$ \\
\hline
\end{tabular}




\begin{tabular}{|c|c|c|c|}
\hline 0 & HOd & HOJ & 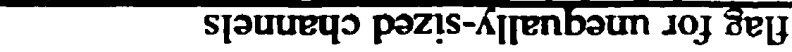 \\
\hline$\overline{0}$ & GHJ & $\overline{\text { QJA }}$ & 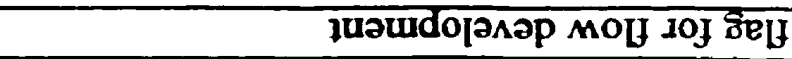 \\
\hline 0 & ODI & ODJ & 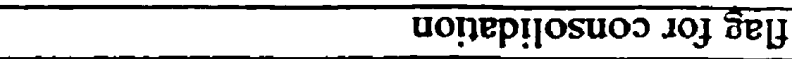 \\
\hline 0 & SXI & SXI & Hoddns jo dol uo suṭlyas soj ฮิeไ \\
\hline 0 & AXI & $\Lambda \mathbf{X J}$ & 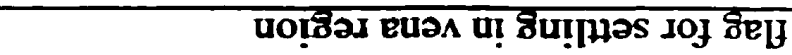 \\
\hline 0 & JXA & त्रJ & 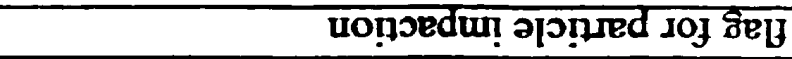 \\
\hline I & W7A & WTI & 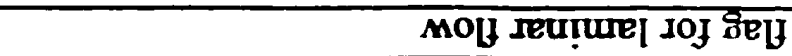 \\
\hline l & Yds & XdJ & 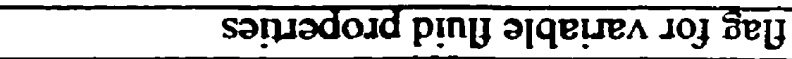 \\
\hline I & X्रDA & YOA & 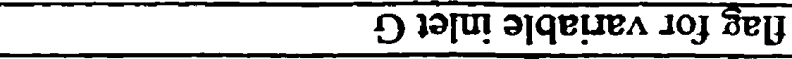 \\
\hline I & $\overline{\text { OGA }}$ & OgA & 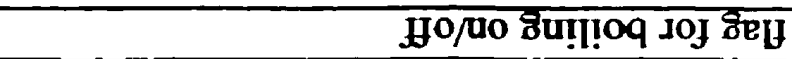 \\
\hline $2 I$ & $\mathbf{I N}$ & & Sdəłs aụ! jo jəqunu \\
\hline 4nuow I & I7Ga & & dəIs aur! jo วZ!s \\
\hline $21-01 \times \varepsilon$ & OV & $\bar{\nabla}$ & V Joloey uo!̣salddns \\
\hline uथा $8 \cdot 2$ & $\mathbf{3}$ & 3 & 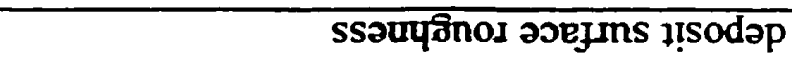 \\
\hline${ }_{c} \mathrm{u} / \mathrm{B} \times \mathrm{X} \quad 00 \mathrm{ZS}$ & SOHA & sd & Kı!suəp l!̣sodəp \\
\hline $\mathbf{X}^{\cdot} \cdot \mathbf{u} / M$ & SNOD & ${ }^{\mathbf{s}_{\mathbf{Y}}}$ & 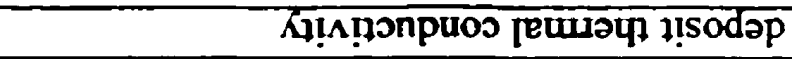 \\
\hline$\varsigma 0$ & SVIJ日 & sg & K!!sojod $7 !$ sodap \\
\hline unu & g8Z & 9 & 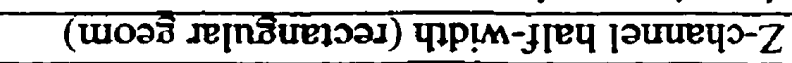 \\
\hline ưtu & $\forall \forall Z$ & $\overline{\mathbf{B}}$ & 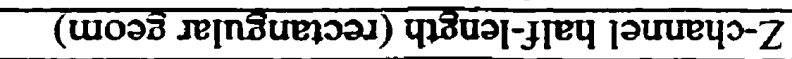 \\
\hline uwu & g8ג & 9 & 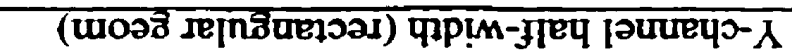 \\
\hline ưu & $\forall \mathbf{V A}$ & $\mathbf{E}$ & 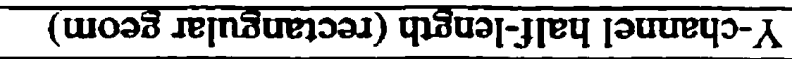 \\
\hline ưu & $\mathrm{OMZ}$ & $M$ & 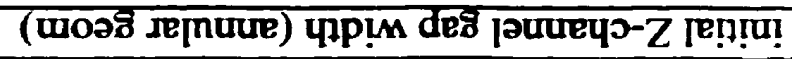 \\
\hline uw & OMA & $\mathbf{M}$ & 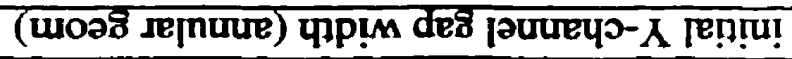 \\
\hline प्wu & $\mathbf{O M}$ & $\mathbf{M}$ & (w0ว8 se[nưue) чpp!м deฮ ueds-22y [e!̣!̣! \\
\hline$L ' Z I$ & OId & ${ }^{39 \pi} \mathrm{d}$ & 1ə1วure!p 2qn1 \\
\hline qdd & OIHd & ${ }^{\top} \phi$ & səjqnjos jo uọ̣enuวJuos \\
\hline un & $\mathrm{sa}$ & ${ }^{\mathbf{s}} \mathbf{d}$ & 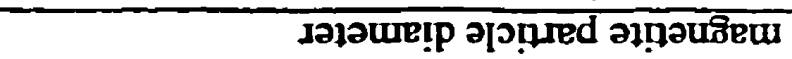 \\
\hline undd & OIHd & $\mathbf{s}_{\boldsymbol{\phi}}$ & 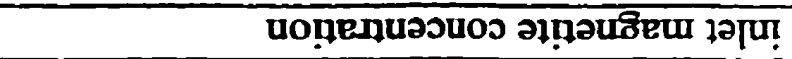 \\
\hline $\mathrm{s}_{2}^{*} \mathbf{w / \delta} \widehat{\mathbf{j}} \quad 00 \varepsilon$ & OD & मण! D & xn!f sseu [e!!!u! \\
\hline$\tau^{\mathrm{t} / M Y} \varepsilon \subseteq Z I$ & 0 & $\mathrm{O}$ & XnI IEZY \\
\hline So $\quad S \angle Z$ & ISI & ${ }^{n} \mathrm{~L}$ & amıejadual วoejuns aqu \\
\hline $9 S 2$ & dSI & ${ }^{n} I$ & 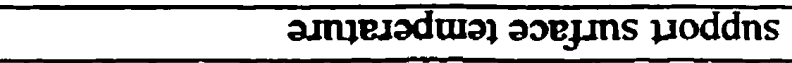 \\
\hline $9 S 2$ & $I$ & ${ }^{9} \mathrm{I}$ & (IESL IE) amlejadual XInq \\
\hline $\mathbf{E}_{\mathbf{d W}}$ & d & d & 2mssaId 1ə[U! \\
\hline$\overline{8 x} / \overline{\mathbf{d x}}$ & OHIX & $4 X$ & Кџ! \\
\hline sịun z̧ әnjen & $\begin{array}{l}\text { ThOHSI } \\
\text { u! loquirs }\end{array}$ & $\begin{array}{c}\text { 1Xə) } \\
\text { u! joquaís }\end{array}$ & uoṇdụssad \\
\hline
\end{tabular}

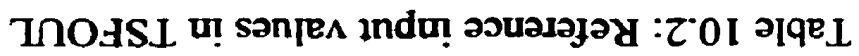


Table 10.3: Predicted values of $\mathrm{K}$ with classic deposition models

(for runs in Figure 10.1a to $\mathrm{e}, \mathrm{t}=12$ months)

NOTE: All the values in the following table are in units of $\mathrm{m} / \mathrm{s}$ and should be multiplied by a factor $1 \times 10^{-6}$. They are shown in this format to make comparisons easier.

\begin{tabular}{|c|c|c|c|c|}
\hline $\begin{array}{c}\text { Deposition } \\
\text { coefficient }\end{array}$ & $\begin{array}{c}\text { free-span region } \\
\text { Node 1 } \\
\text { (on tube) }\end{array}$ & $\begin{array}{c}\text { support region } \\
\text { Node 21 } \\
\text { (on tube) }\end{array}$ & $\begin{array}{c}\text { support region } \\
\text { Node 21 } \\
\text { (on support) }\end{array}$ & $\begin{array}{c}\text { free-span region } \\
\text { Node 65 } \\
\text { (on tube) }\end{array}$ \\
\hline $\mathrm{K}_{\mathrm{TH}}$ & -0.08 & -0.08 & 0 & -0.08 \\
\hline $\mathrm{K}_{\mathrm{D}}$ & 2.3 & 13 & 13 & 2.3 \\
\hline $\mathrm{K}_{\mathrm{I}}$ & 1.1 & 430 & 430 & 1.2 \\
\hline $\mathrm{K}_{\mathrm{T}}$ & 2.3 & 440 & 440 & 2.4 \\
\hline $\mathrm{K}_{\mathrm{A}}$ & 0.84 & 1400000 & 1400000 & 0.86 \\
\hline $\mathrm{K}_{\mathrm{B}}$ & 29 & 41 & 0 & 29 \\
\hline $\mathrm{K}_{1 \phi}$ & 0.62 & 440 & 440 & 0.63 \\
\hline $\mathrm{K}_{2 \phi}$ & 29 & 480 & 0 & 30 \\
\hline $\mathrm{K}_{\mathrm{F}}$ & 0 & 0 & 0 & 0 \\
\hline $\mathrm{K}_{\mathrm{V}}$ & 0 & 0 & 0 & 0 \\
\hline $\mathrm{K}_{\mathrm{G}}$ & 0 & 0 & 0 & 0 \\
\hline $\mathrm{K}_{\mathrm{C}}$ & 0 & 0 & 0 & 0 \\
\hline $\mathrm{K}_{\mathrm{overall}}$ & 29 & 480 & 440 & 30 \\
\hline
\end{tabular}

$\mathrm{K}_{\mathrm{TH}}$ thermophoresis

$K_{D} \quad$ diffusion

$K_{I} \quad$ inertial coasting

$\mathrm{K}_{2 \phi}$ two-phase

$\mathrm{K}_{\mathrm{T}}$ transport

$\mathrm{K}_{\mathrm{F}} \quad$ impaction

$\mathrm{K}_{\mathrm{A}}$ attachment

$\mathrm{K}_{V} \quad$ centrifugal settling in vena region

$\mathrm{K}_{\mathrm{B}} \quad$ boiling

$\mathrm{K}_{1 \phi} \quad$ single-phase

$\mathrm{K}_{\mathrm{G}} \quad$ gravitational settling

$K_{C} \quad$ centrifugal settling

$K_{\text {overal! }}$ either $K_{1 \phi}$ or $K_{2 \phi}$ 
Table 10.4: Input values for simulation of bi-foil experiment

\begin{tabular}{|c|c|}
\hline Description & value \& units \\
\hline inlet steam quality & $0 \quad \mathrm{~kg} / \mathrm{kg}$ \\
\hline inlet pressure & $\mathbf{M P a}$ \\
\hline bulk temperature (at Tsat) & 270 \\
\hline support surface temperature & 270 \\
\hline tube surface temperature & 270 \\
\hline heat flux & $\mathrm{kW} / \mathrm{m}^{2}$ \\
\hline initial mass flux & $72.3 \mathrm{~kg} / \mathrm{m}^{2} . \mathrm{s}$ \\
\hline inlet magnetite concentration & ppm \\
\hline magnetite particle diameter & $0.25 \mu \mathrm{m}$ \\
\hline concentration of solubles & $\mathrm{ppb}$ \\
\hline tube diameter & $12.7 \mathrm{~mm}$ \\
\hline initial free-span gap width (annular geom) & 13.7 \\
\hline initial Y-channel gap width (annular geom) & $1.34 \mathrm{~mm}$ \\
\hline initial Z-channel gap width (annular geom) & 1.34 \\
\hline Y-channel half-length (rectangular geom) & $\mathbf{m m}$ \\
\hline Y-channel half-width (rectangular geom) & $\mathbf{m m}$ \\
\hline Z-channel half-length (rectangular geom) & $\mathrm{mm}$ \\
\hline Z-channel half-width (rectangular geom) & $\mathrm{mm}$ \\
\hline deposit porosity & 0.5 \\
\hline deposit thermal conductivity & $20 \quad$ W/m.K \\
\hline deposit density & $5200 \mathrm{~kg} / \mathrm{m}^{3}$ \\
\hline deposit surface roughness & $2.8 \mu \mathrm{m}$ \\
\hline suppression factor $A$ & $3 \times 10^{-12}$ \\
\hline size of time step & 0.139 months \\
\hline number of time steps & 6 \\
\hline flag for boiling on/off (FBO) & 0 \\
\hline flag for variable inlet $G$ (FGK) & 0 \\
\hline flag for variable fluid properties (FPR) & 0 \\
\hline flag for laminar flow (FLM) & 0 or 1 \\
\hline flag for particle impaction (FKF) & 0 or 1 \\
\hline flag for settling in vena region (FKV) & 0 or 1 \\
\hline flag for settling on top of support (FKS) & 0 or 1 \\
\hline flag for consolidation (FCO) & 0 or 1 \\
\hline flag for flow development (FFD) & 0 of 1 \\
\hline flag for unequally-sized channels (FCH) & 0 \\
\hline
\end{tabular}


Figure 10.1: Classic models: reference case

(a) predicted deposition profiles

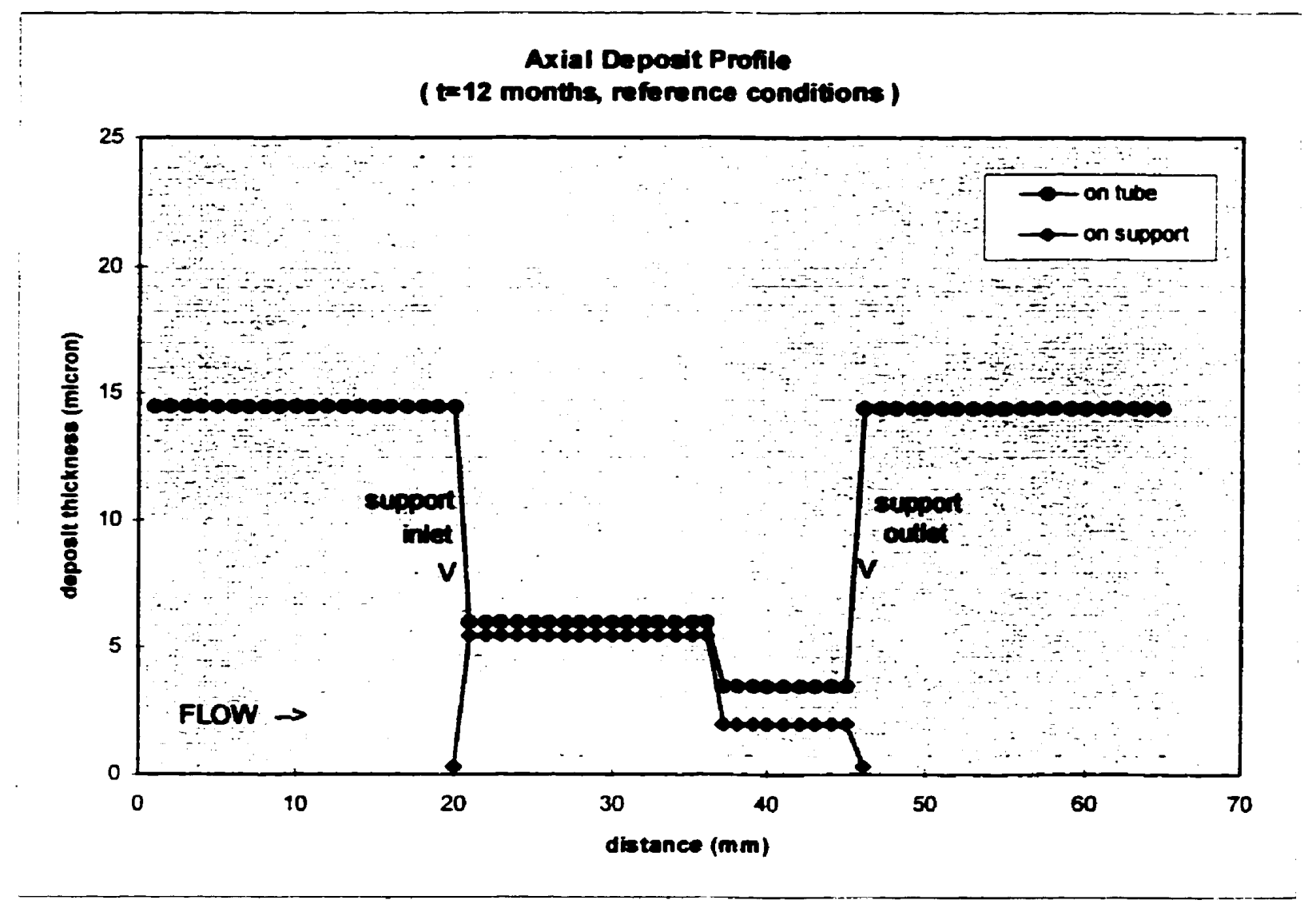

Input values:

Reference values as per Table 10.2. 
Figure 10.1 (continued)

(b) growth of deposit with time

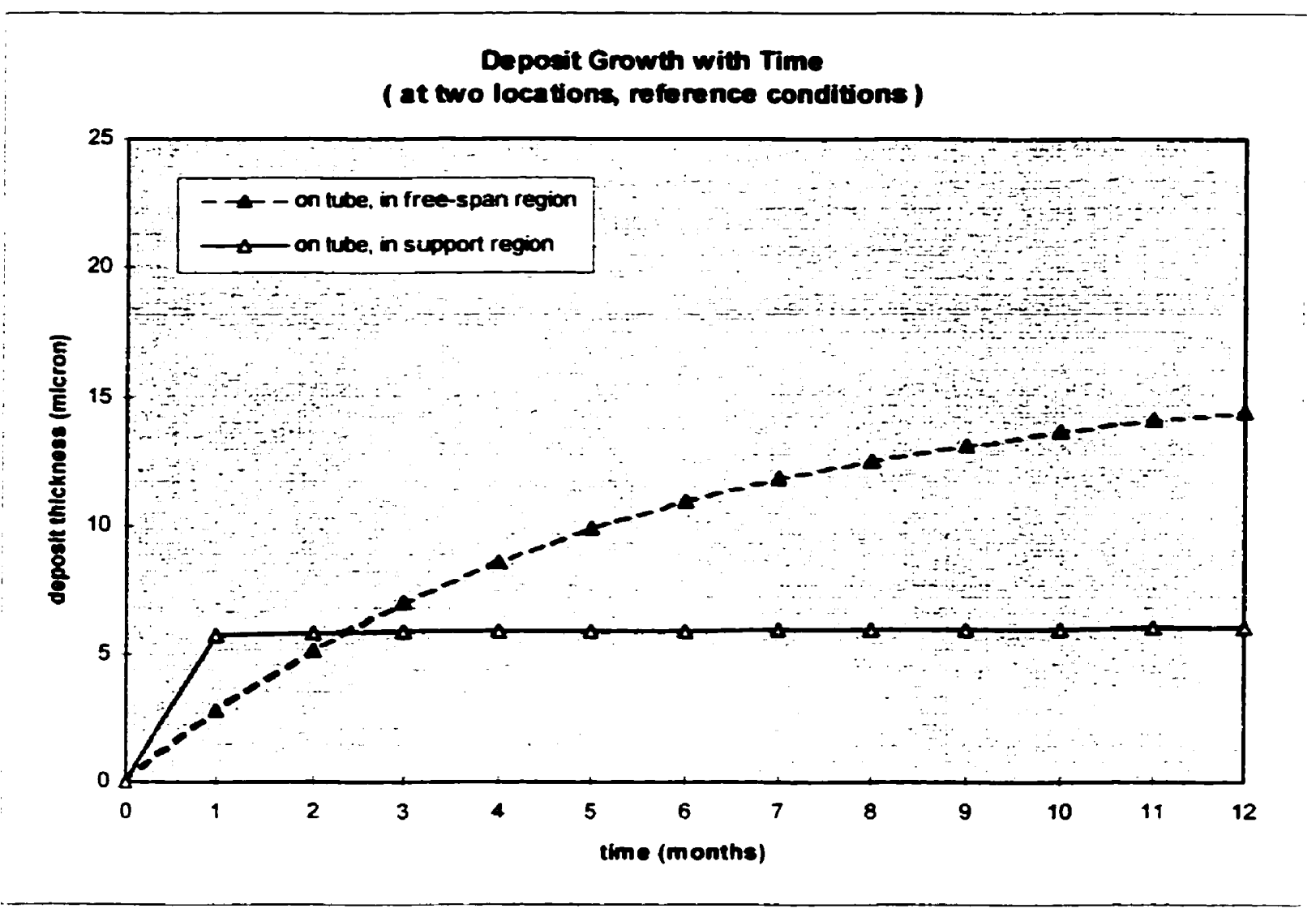

Input values:

Reference values as per Table 10.2. 
Figure 10.2: Sensitivity runs with classic models

(a) sensitivity with mass flux G

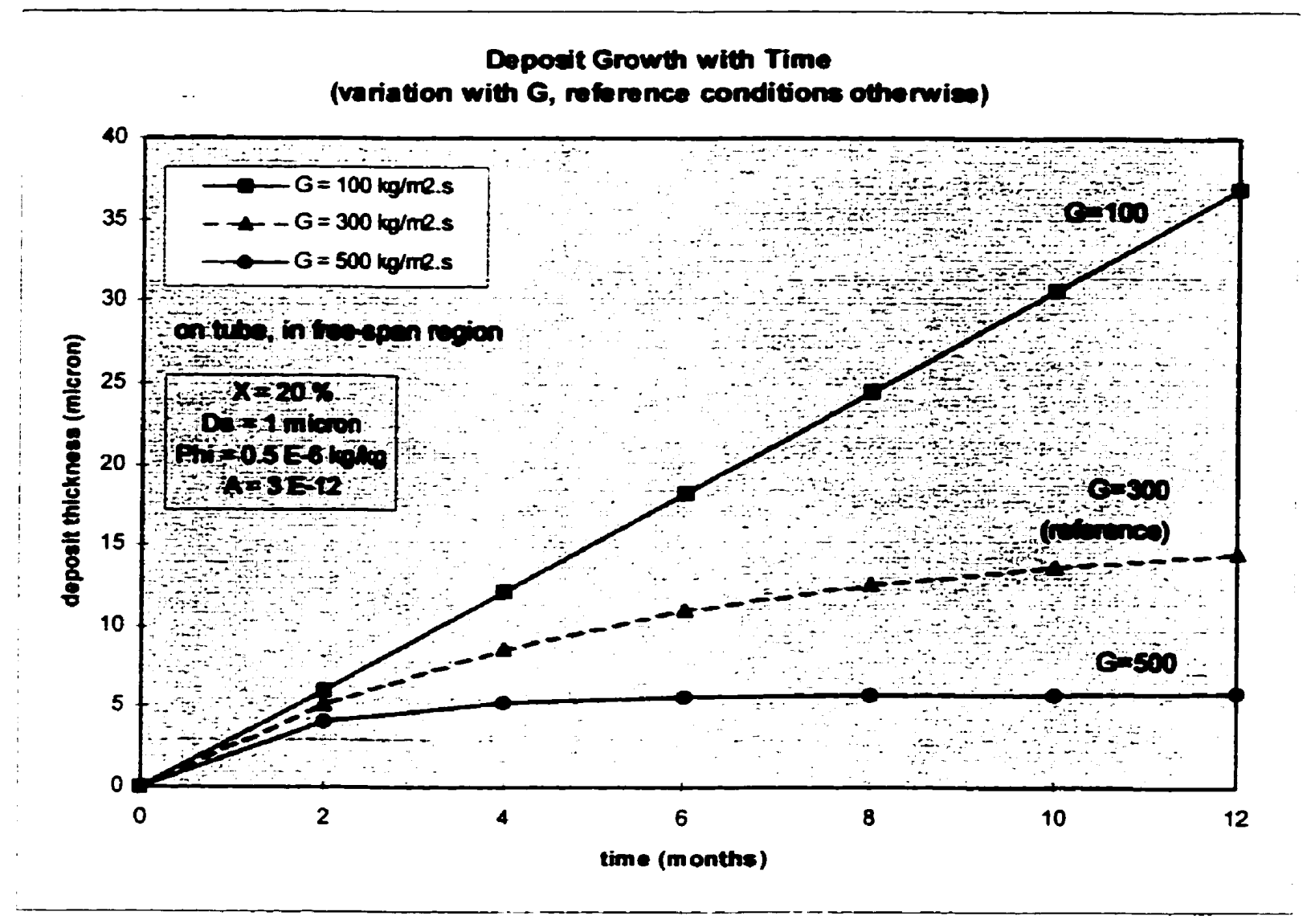

Input values:

initial inlet mass flux all other values:

$100,300,500 \quad \mathrm{~kg} / \mathrm{m}^{2} . \mathrm{s}$ reference values as per Table 10.2 
Figure 10.2 (continued)

(b) sensitivity with steam quality $\chi$

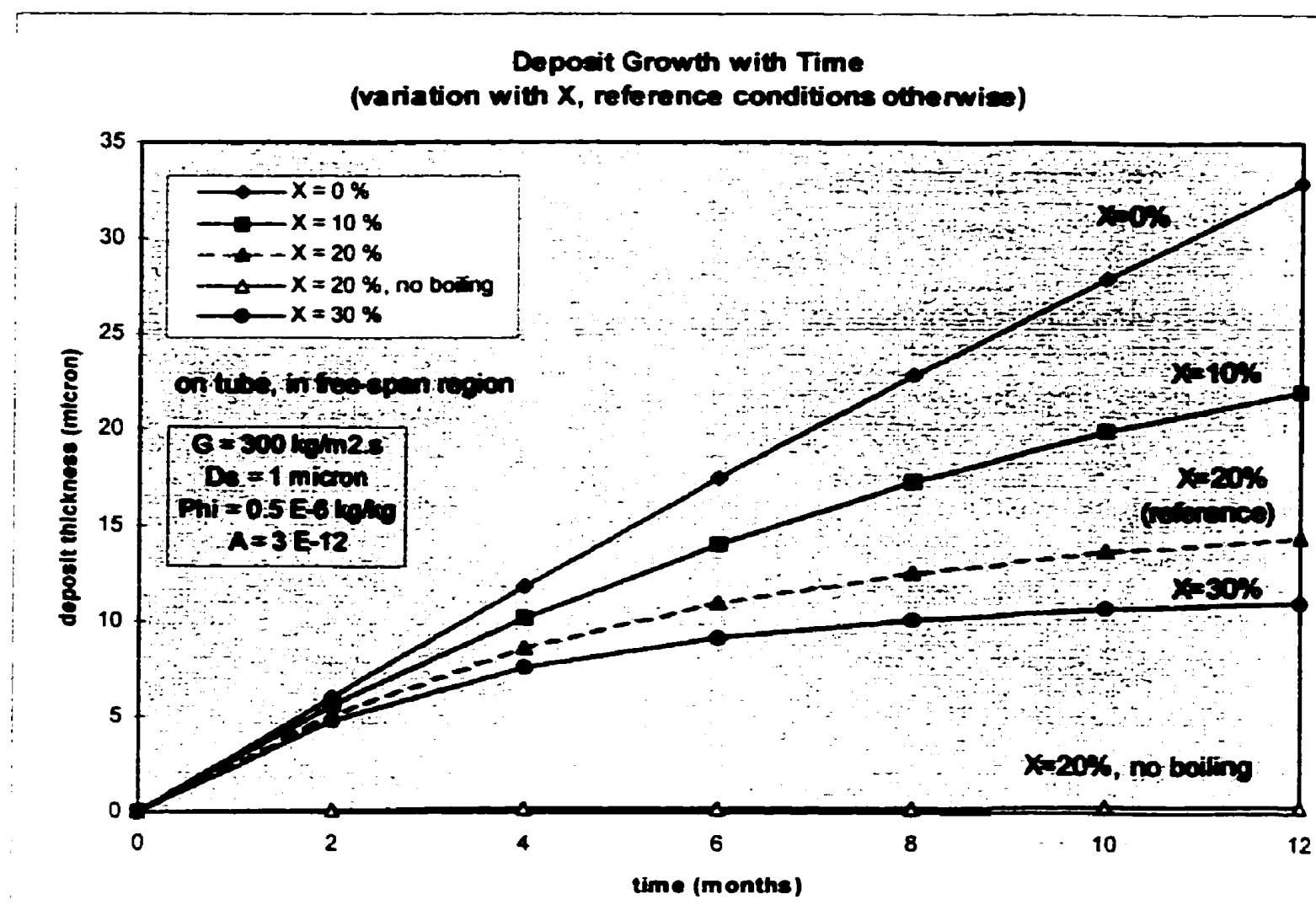

Input values:

inlet quality

flag for boiling FBO

$0,10,20,30 \%$

$\mathrm{kg} / \mathrm{kg}$

all other values:

0 for the "no boiling" run only reference values as per Table 10.2 
Figure 10.2 (continued)

(c) sensitivity with particle concentration $\phi$

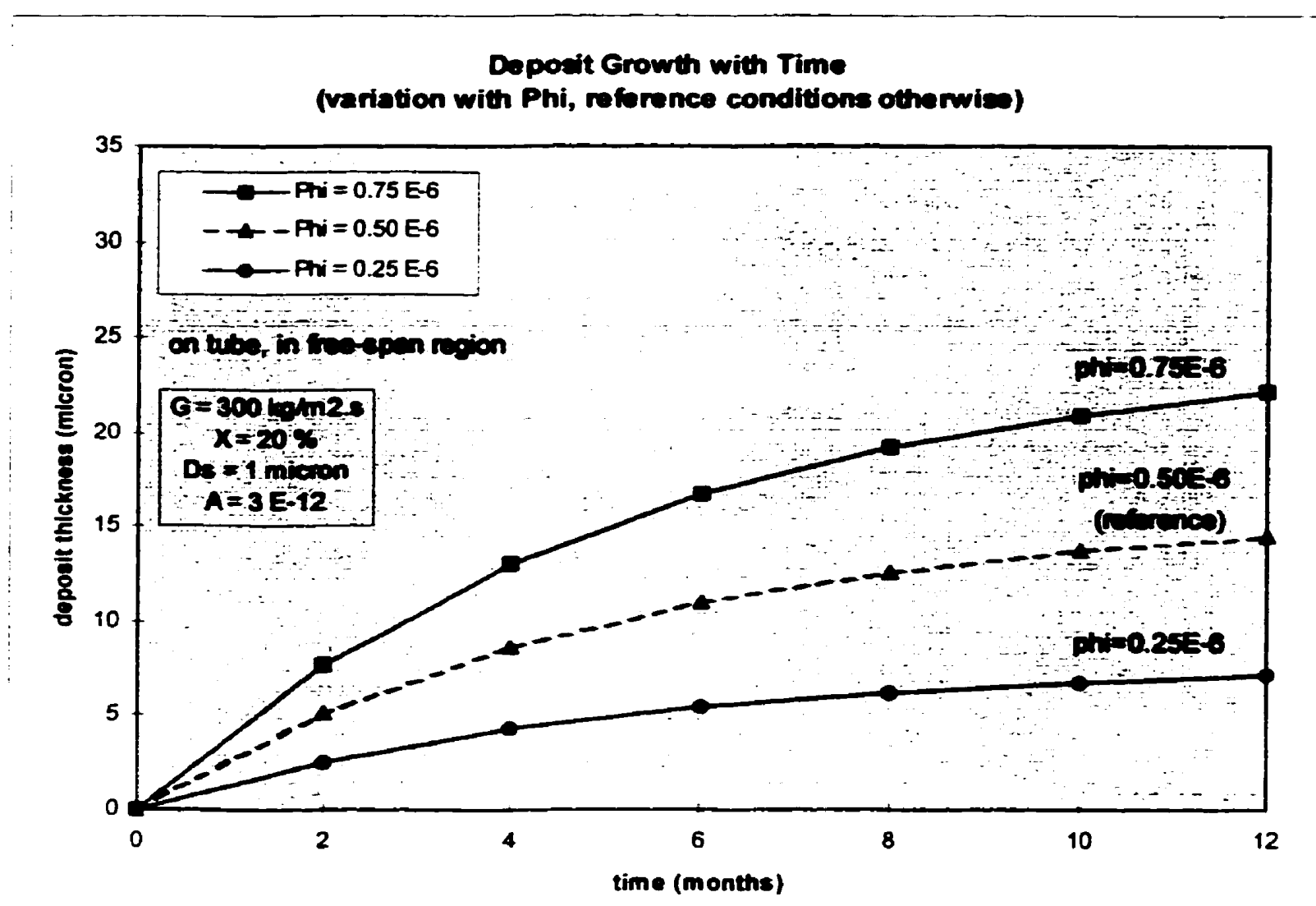

Input values:

inlet particle conc.

$0.25,0.50,0.75$

ppm

all other values:

reference values as per Table 10.2 
Figure 10.2 (continued)

(d) sensitivity with particle diameter $D_{s}$

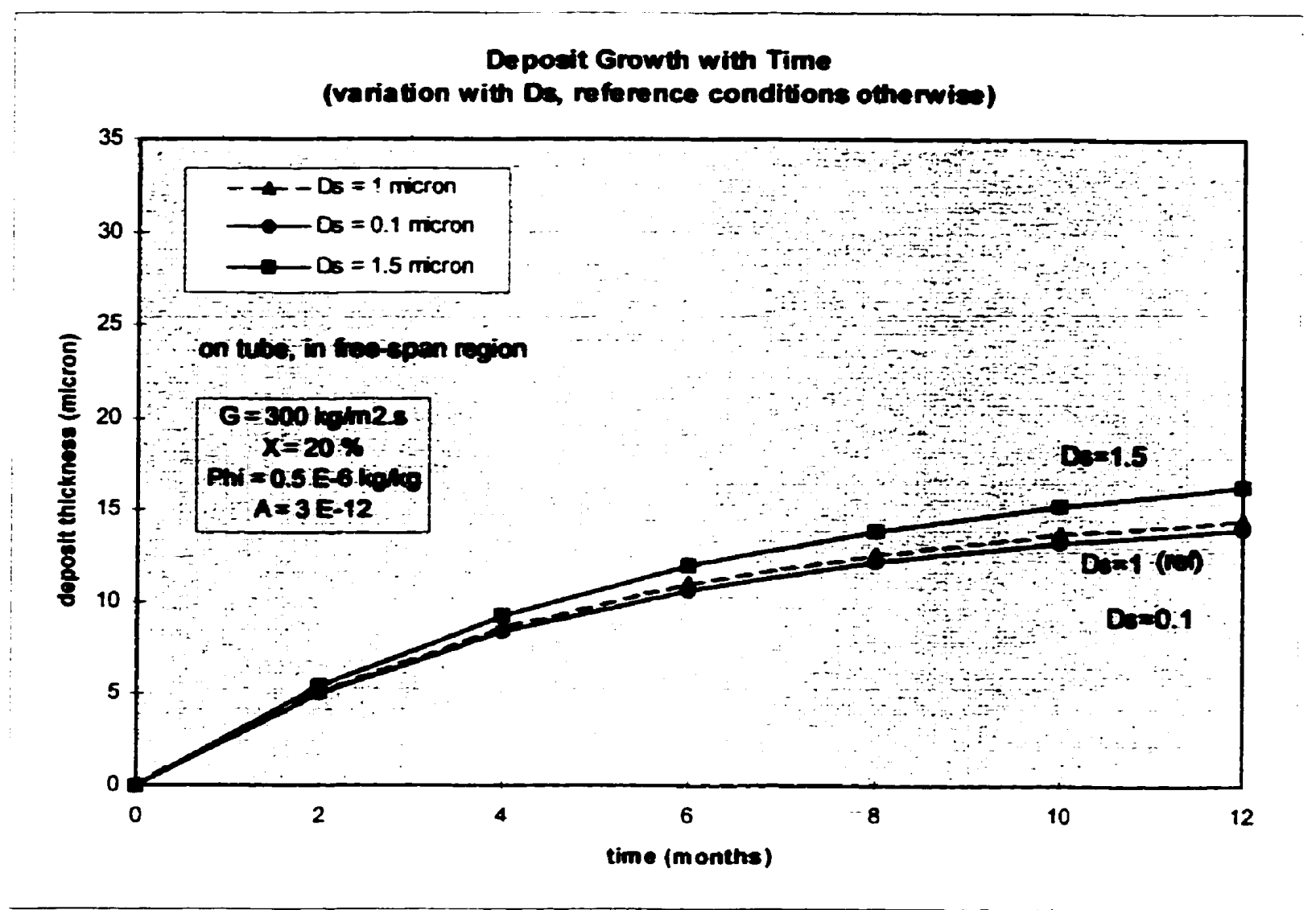

Input values: particle diameter $0.1,1,1.5$ micron all other values: reference values as per Table 10.2 
Figure 10.2 (continued)

(e) sensitivity with suppression factor A

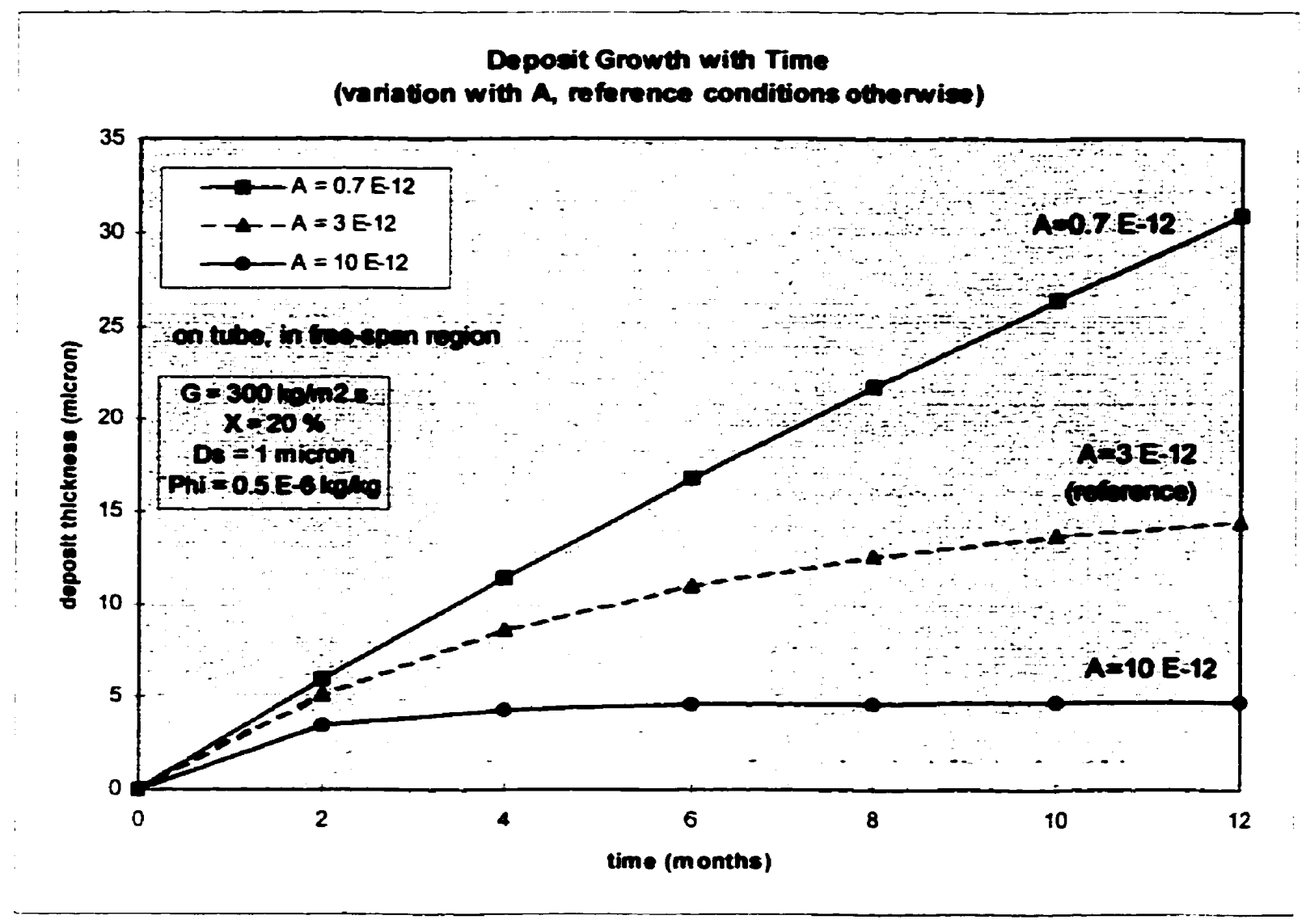

Input values:

suppression factor

$0.7 \times 10^{-12}, 3 \times 10^{-12}, 10 \times 10^{-12}$

all other values:

reference values as per Table 10.2 
Figure 10.3: Effect of flow regime

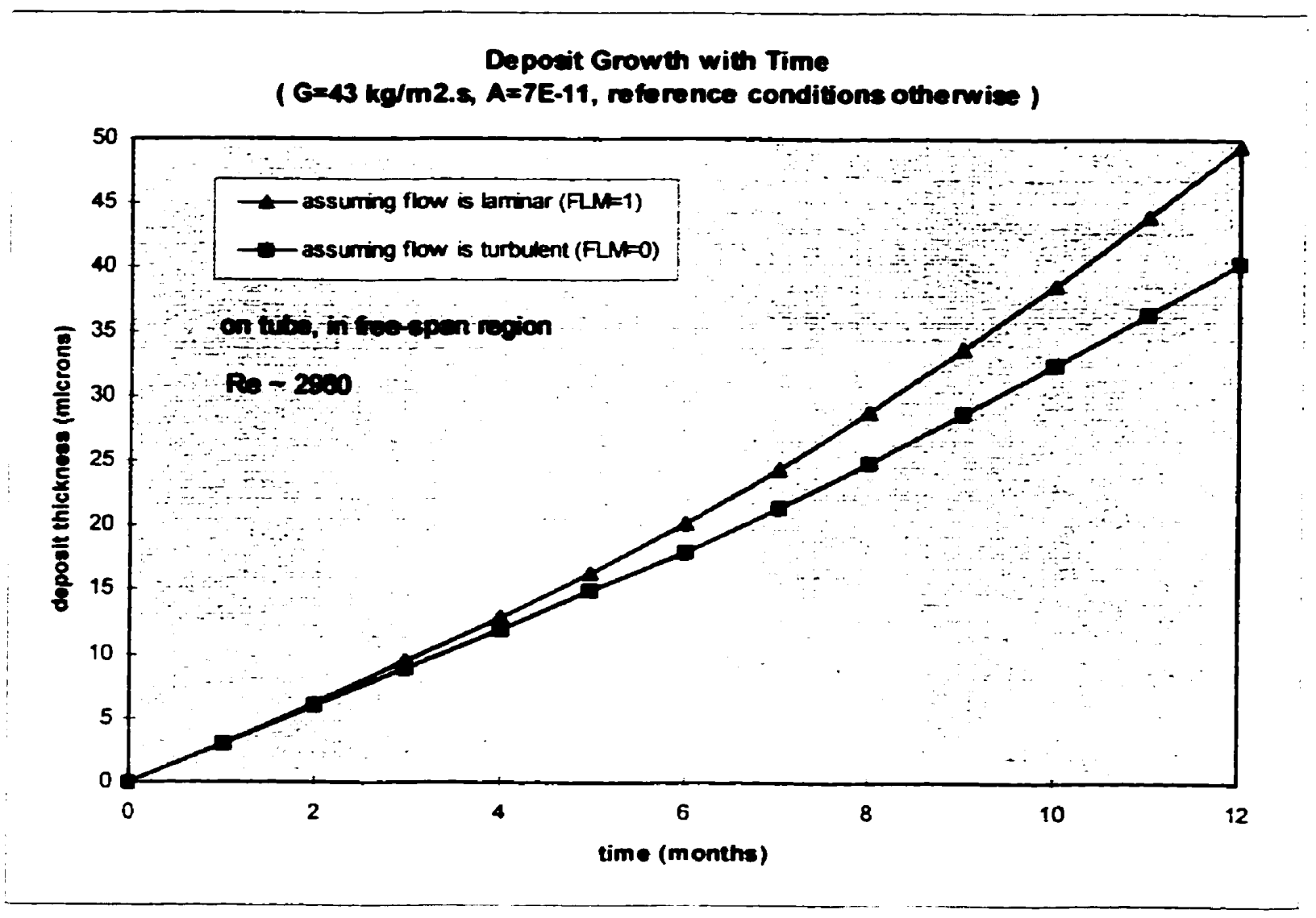

Input values:

initial inlet mass flux suppression factor

$43 \mathrm{~kg} / \mathrm{m}^{2} . \mathrm{s}$

flag for flow regime FLM

$7 \times 10^{-11}$

all other values:

I or 0

reference values as per Table 10.2 
Figure 10.4: Effect of particle impaction

(a) deposit growth with time

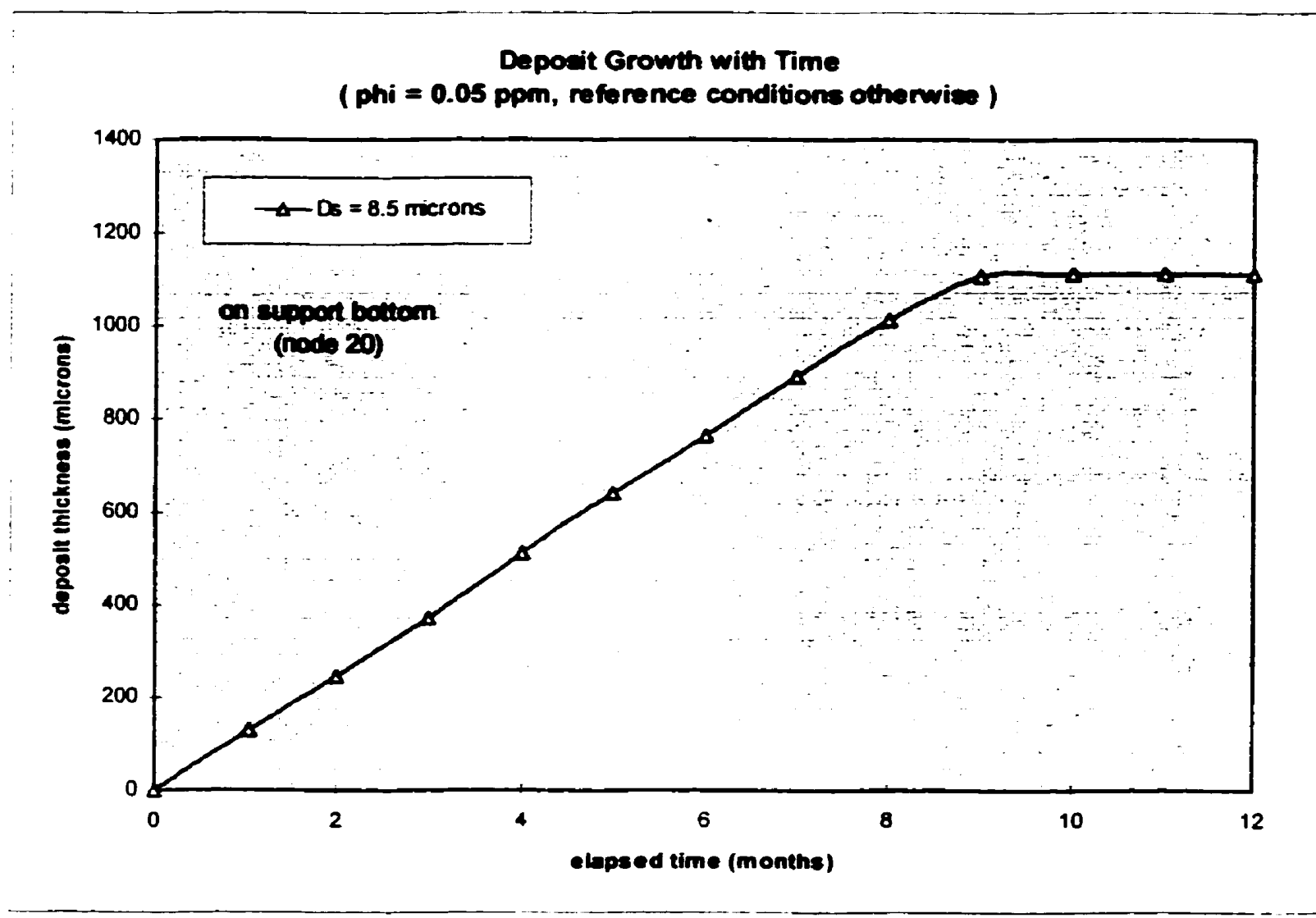

Input values:

inlet particle concentration

0.05 ppm

particle diameter

8.5 microns

flag for particle impaction FKF

1

all other values:

reference values as per Table 10.2 
Figure 10.4 (continued)

(b) deposit thickness as a function of particle size

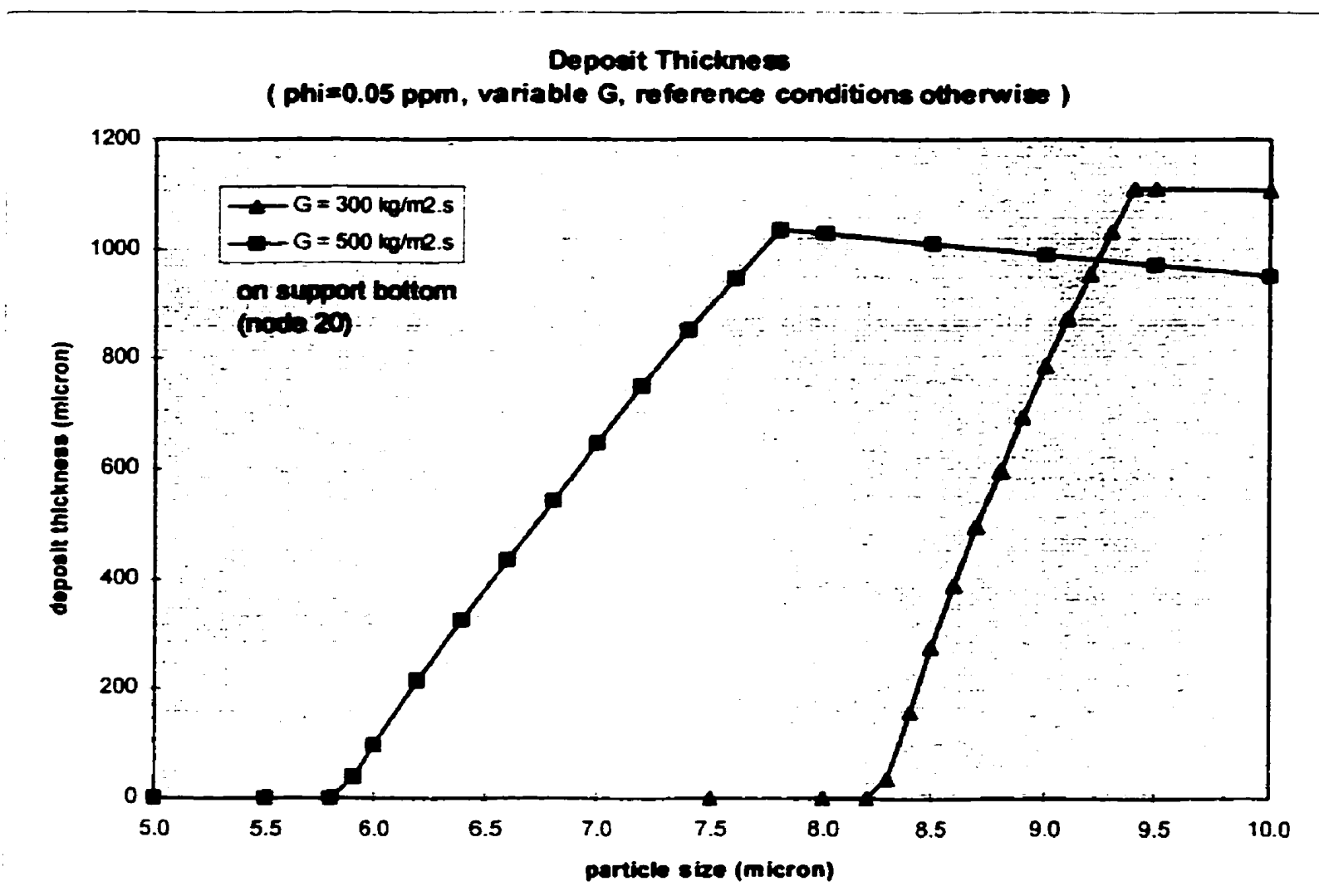

Input values:

initial inlet mass flux inlet particle concentration particle diameter time period

300,500 $\mathrm{kg} / \mathrm{m}^{2} . \mathrm{s}$

0.05 ppm variable 2 months flag for particle impaction FKF 1 all other values: reference values as per Table 10.2 
Figure 10.5: Effect of settling

(a) axial deposit profile

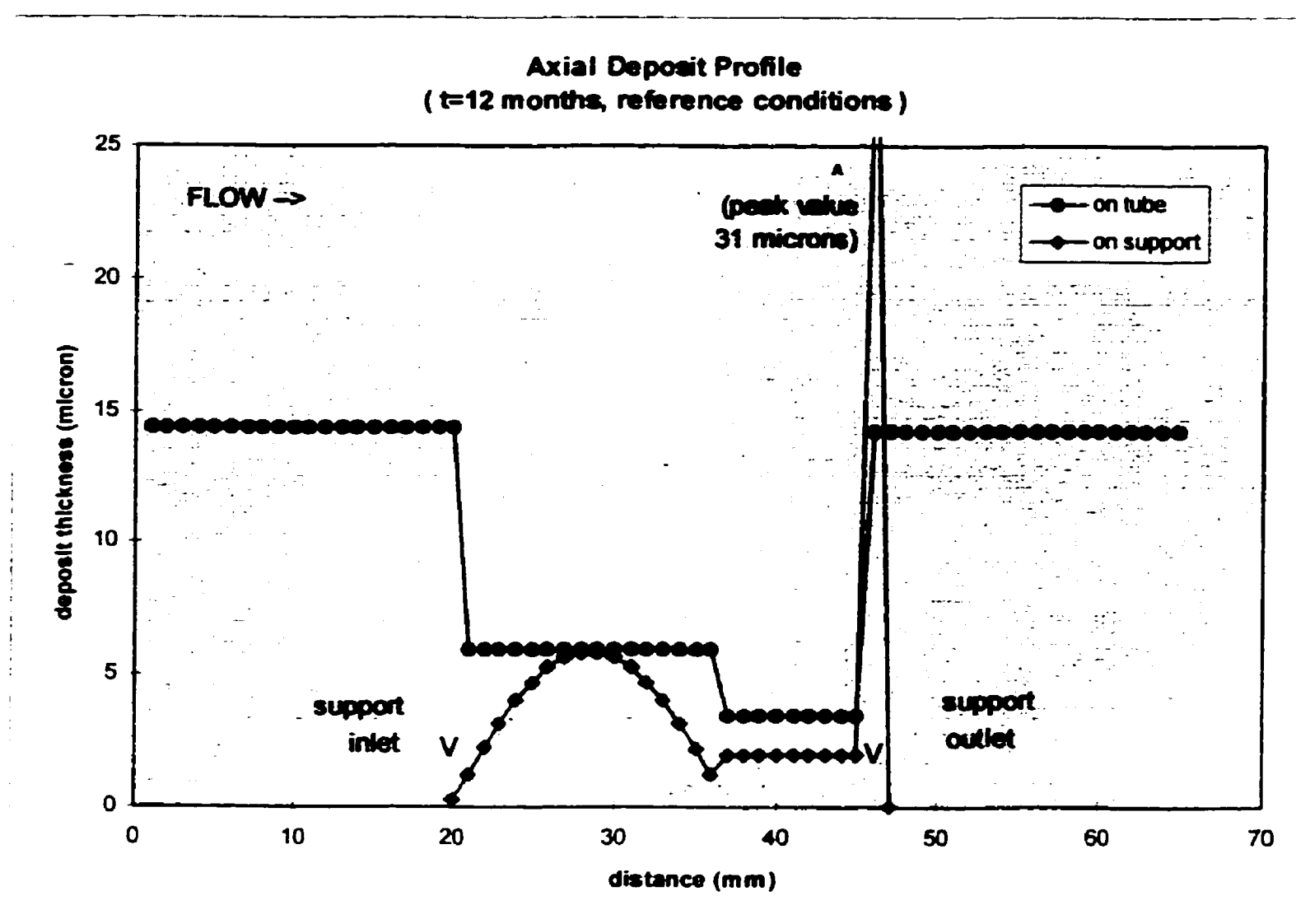

Input values:

flag for settling in vena region FKV 1 flag for settling on top of support FKS 1 all other values: reference values as per Table 10.2 
Figure 10.5 (continued)

(b) deposit thickness as a function of particle size

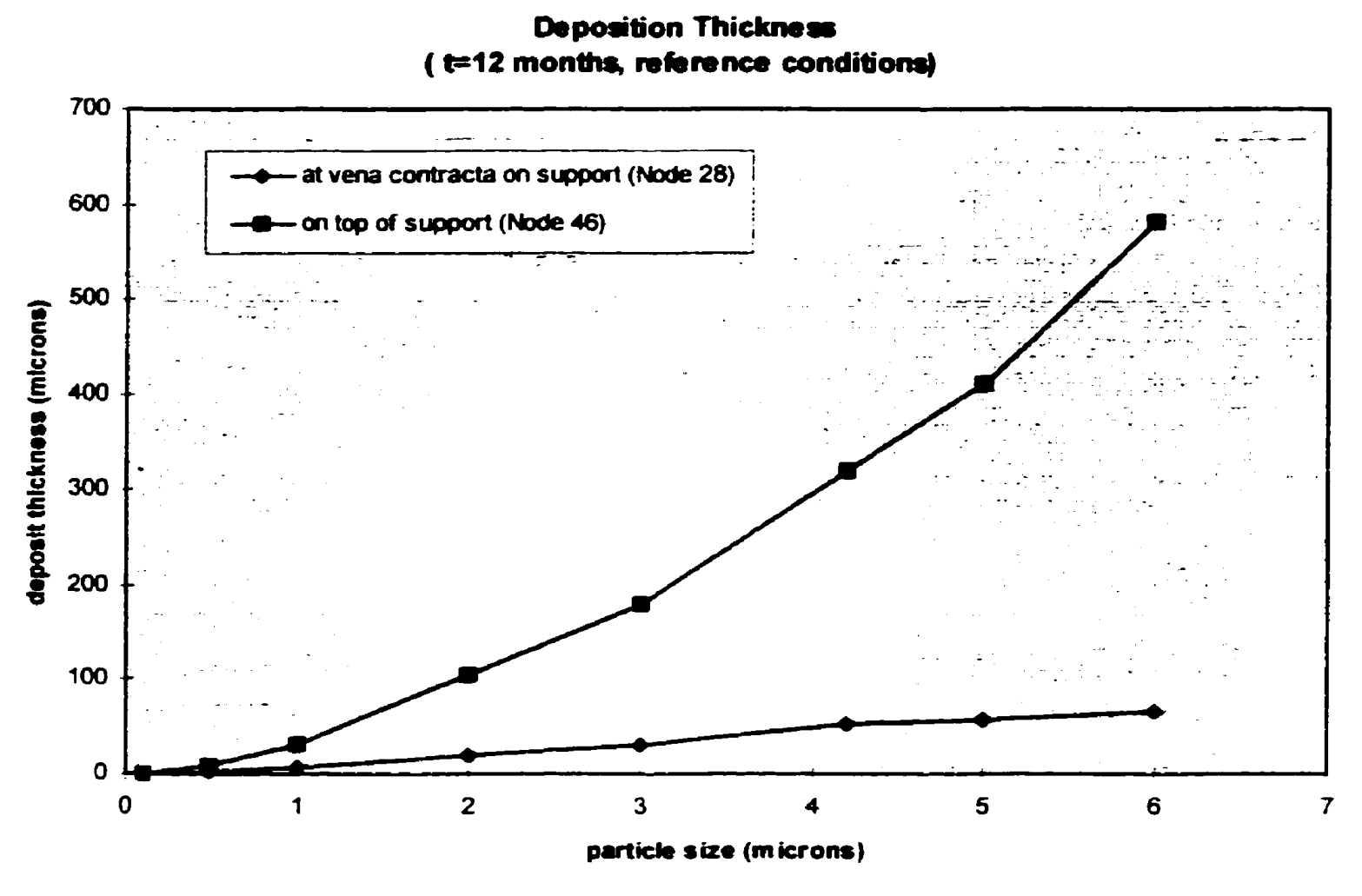

Input values:

particle diameter

flag for settling in vena region FKV

variable

flag for settling on top of support FKS

all other values:

1

reference values as per Table 10.2 
Figure 10.6: Effect of flashing and consolidation

(a) variation of $\mathrm{A}$ with axial distance

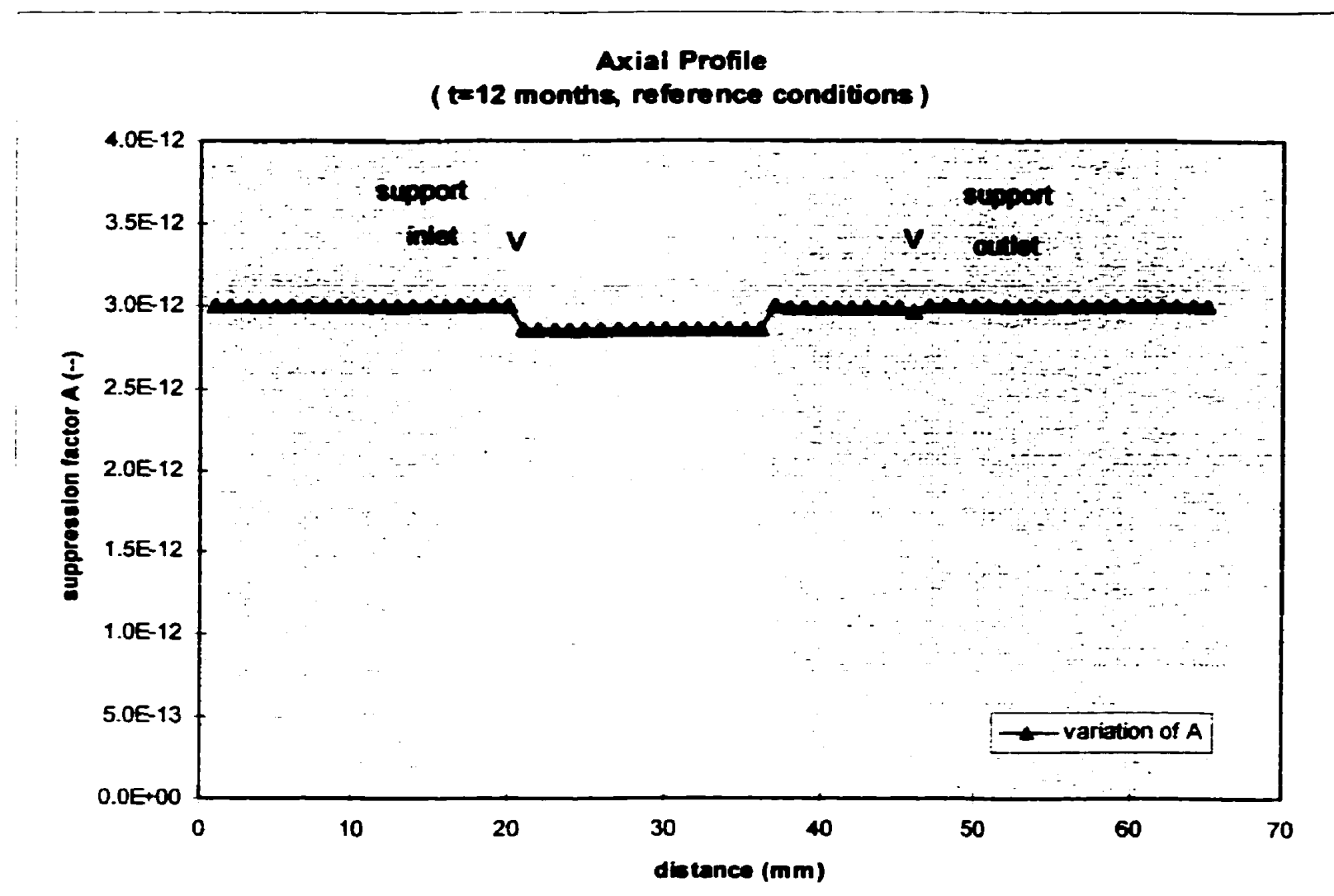

Input values:

concentration of solubles

flag for consolidation FCO

1000 ppb

all other values:

1

reference values as per Table $\mathbf{1 0 . 2}$ 
Figure 10.6 (continued)

(b) axial pressure profile

Axial Preseure Profile

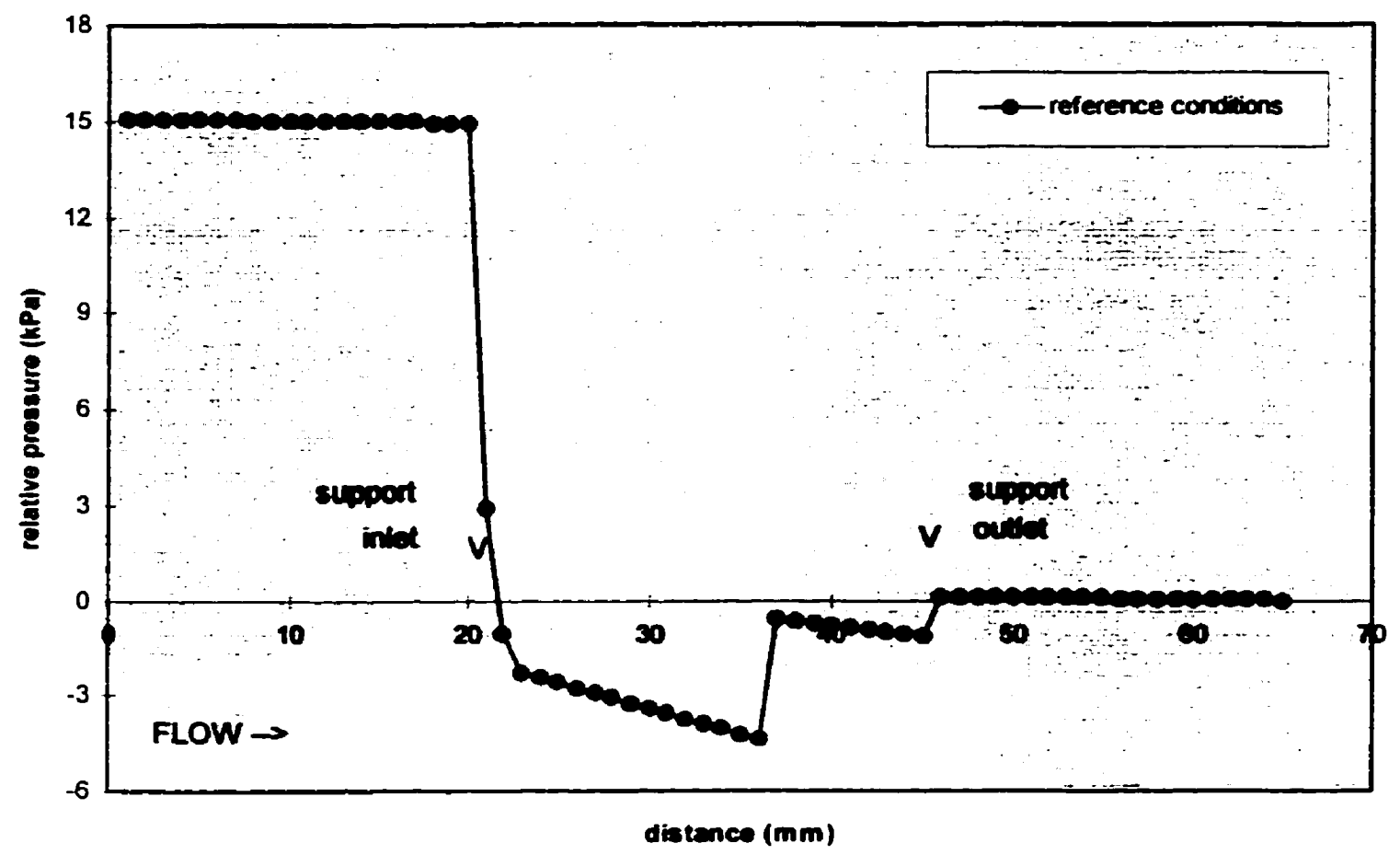

Input values:

reference values as per Table 10.2 
Figure 10.6 (continued)

(c) axial deposit profile

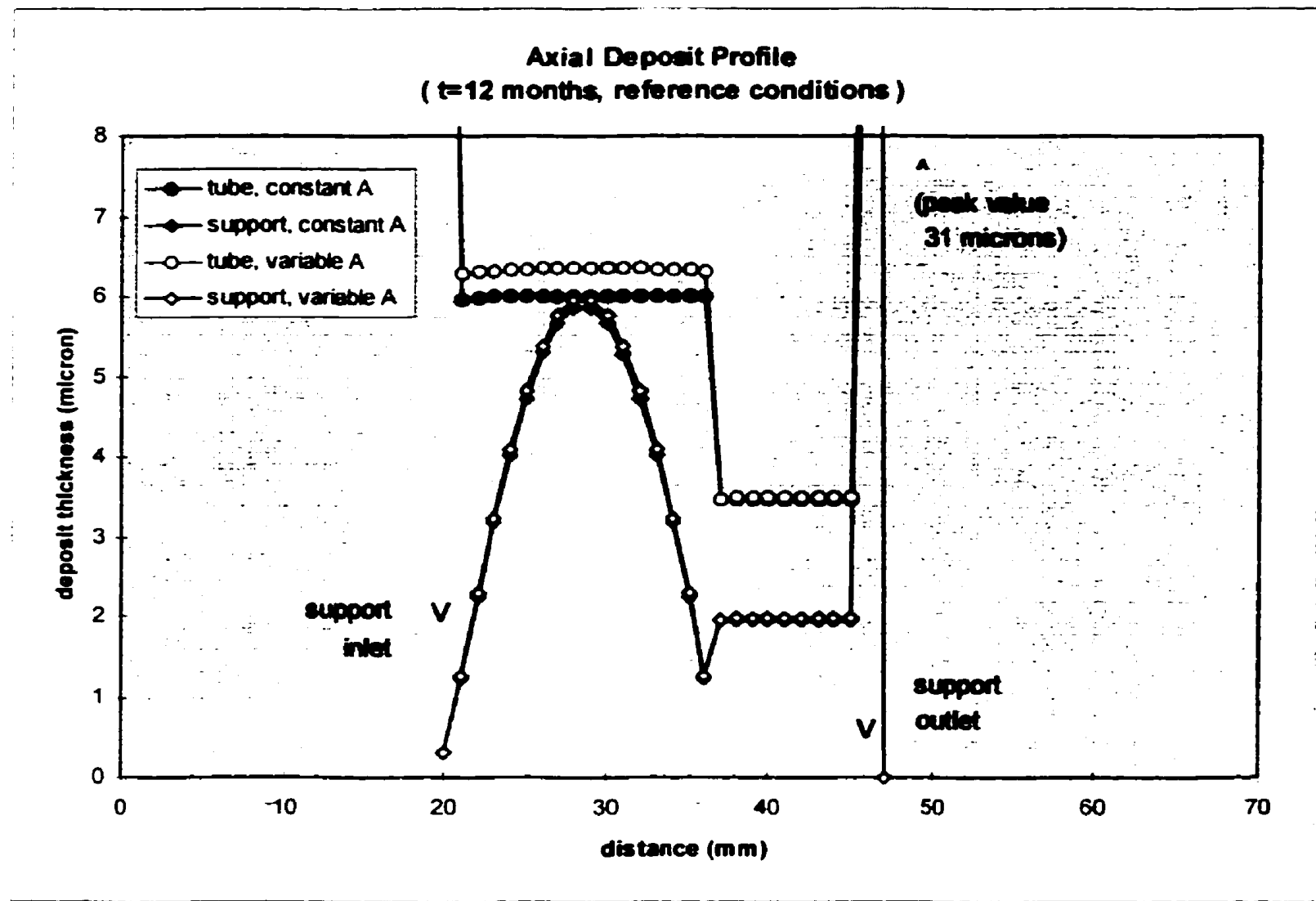

Input values:

concentration of solubles

flag for settling in vena region FKV

$1000 \mathrm{ppb}$

flag for settling on top of support FKS

flag for consolidation FCO

all other values:

1

1

0 (constant A) or 1 (variable A) reference values as per Table 10.2 
Figure 10.7: Effect of flow development

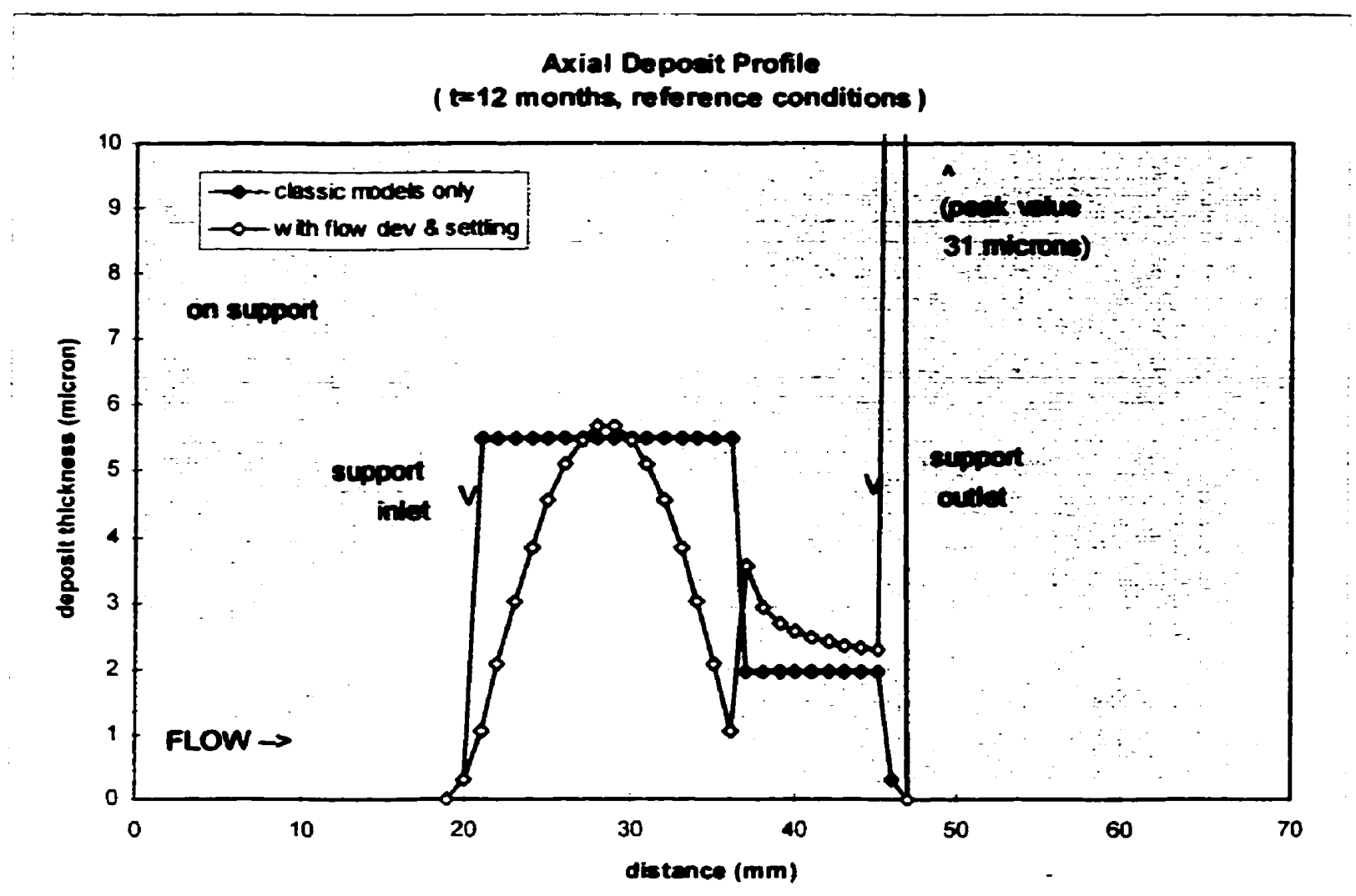

Input values:

flag for settling in vena region FKV flag for settling on top of support FKS flag for flow development FFD all other values:
0 (classic models only) or 1 0 (classic models only) or 1 0 (classic models only) or 1 reference values as per Table 10.2 
Figure 10.8: Effect of unequally-sized channels

(a) void distribution

Void Distribution

( $t=12$ monthe, veriable $Z G=300$ or $80 \mathrm{~kg} / \mathrm{m} 2.8$ )

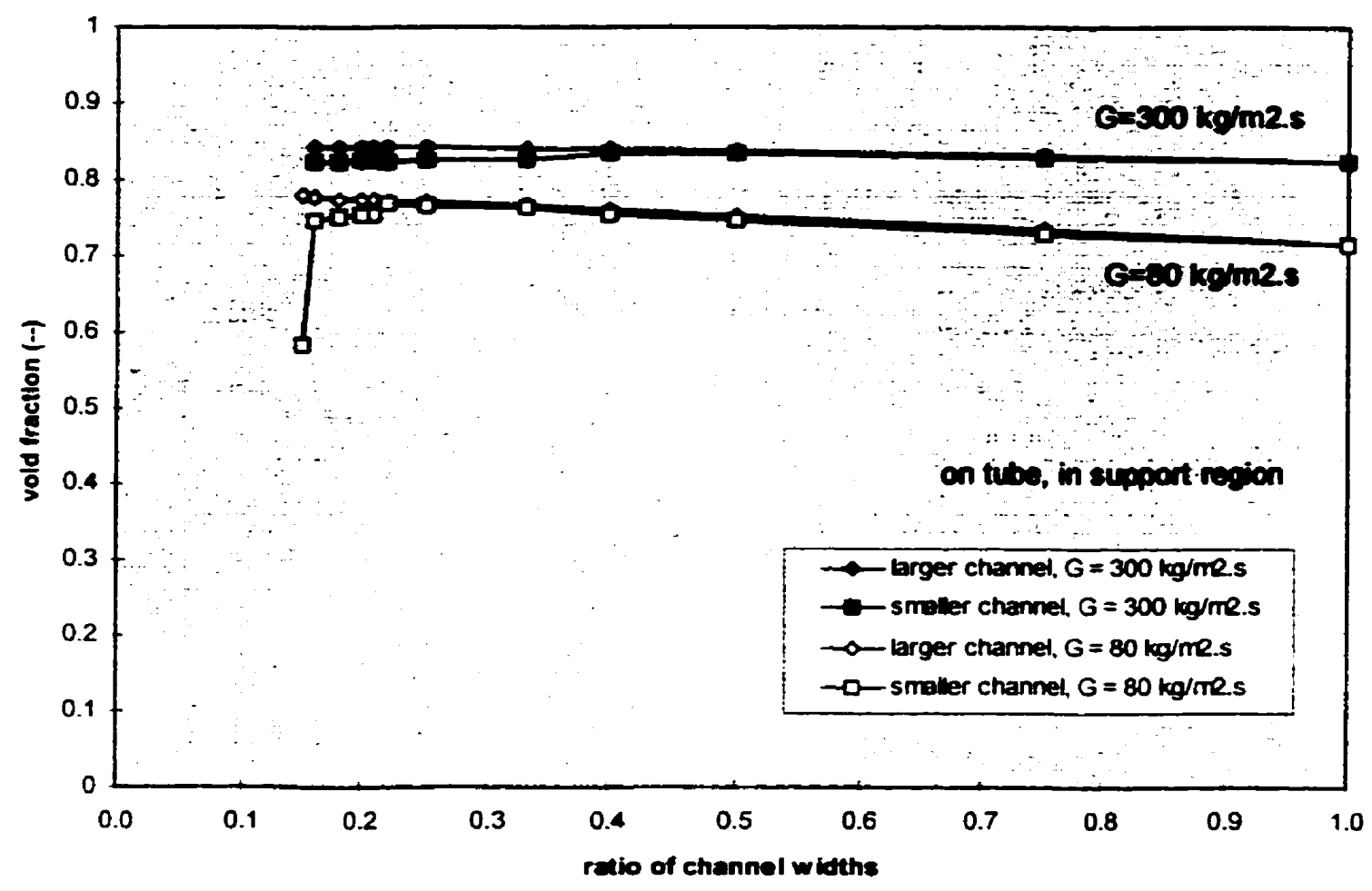

Input values:

initial Z-channel annular gap width initial Z-channel rect. half-width initial mass flux flag for unequal channel sizes FCH all other values: variable, 2.1 to $0.32 \mathrm{~mm}$ variable, 2.1 to $0.32 \mathrm{~mm}$ 80 or $300 \mathrm{~kg} / \mathrm{m}^{2}$.s 1 reference values as per Table 10.2 
Figure 10.8 (continued)

(b) difference in deposit growth, reference mass flux

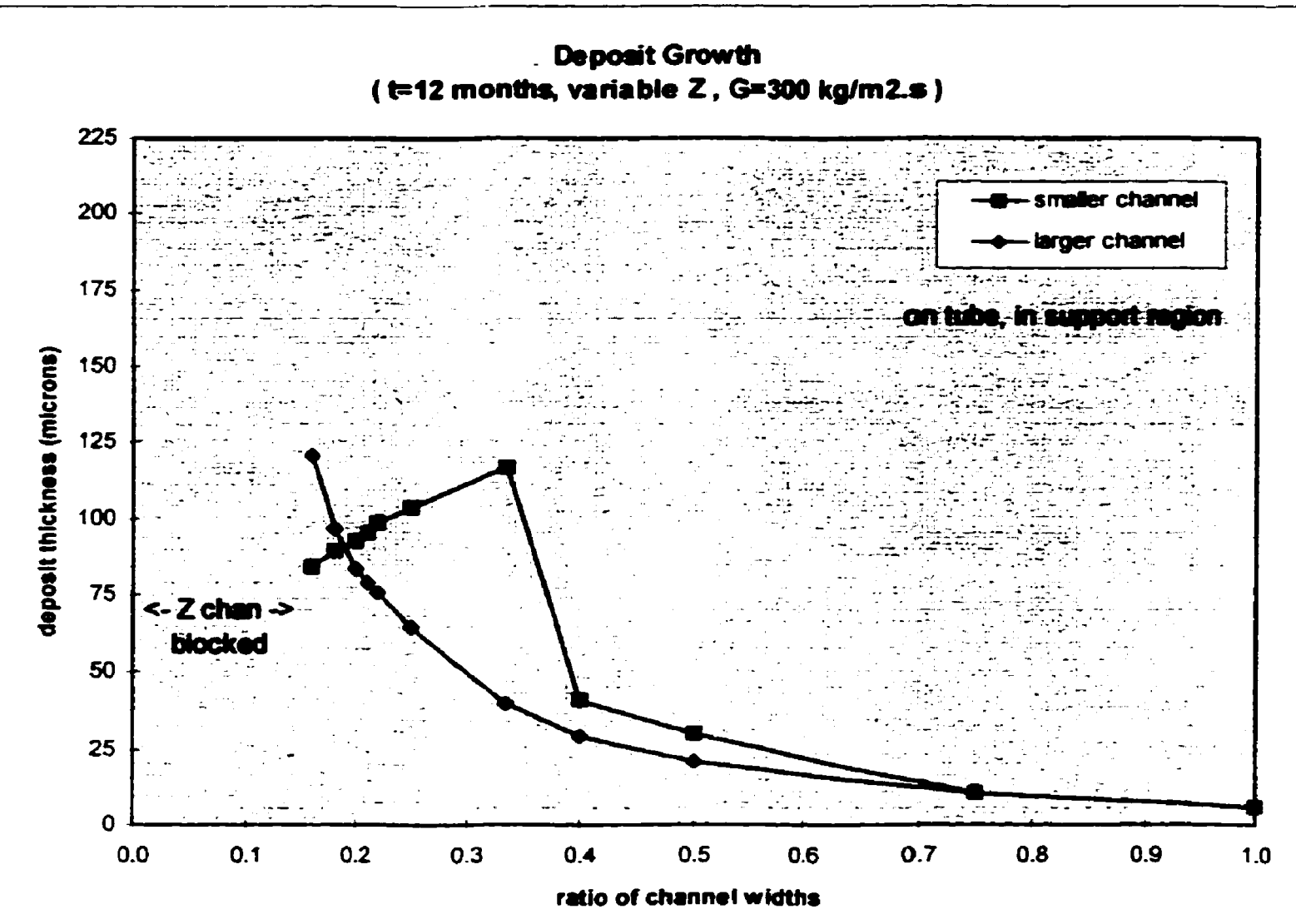

Input values:

initial Z-channel annular gap width initial Z-channel rect. half-width flag for unequal channel sizes $\mathrm{FCH}$ all other values: variable, 2.1 to $0.32 \mathrm{~mm}$ variable, 2.1 to $0.32 \mathrm{~mm}$ 1 reference values as per Table 10.2 
Figure 10.8 (continued)

(c) difference in deposit growth, lower mass flux

\section{Deposit Growth}

( $t=12$ months, variable Z, G=80 $\mathrm{kg} / \mathrm{mz} .=$ )

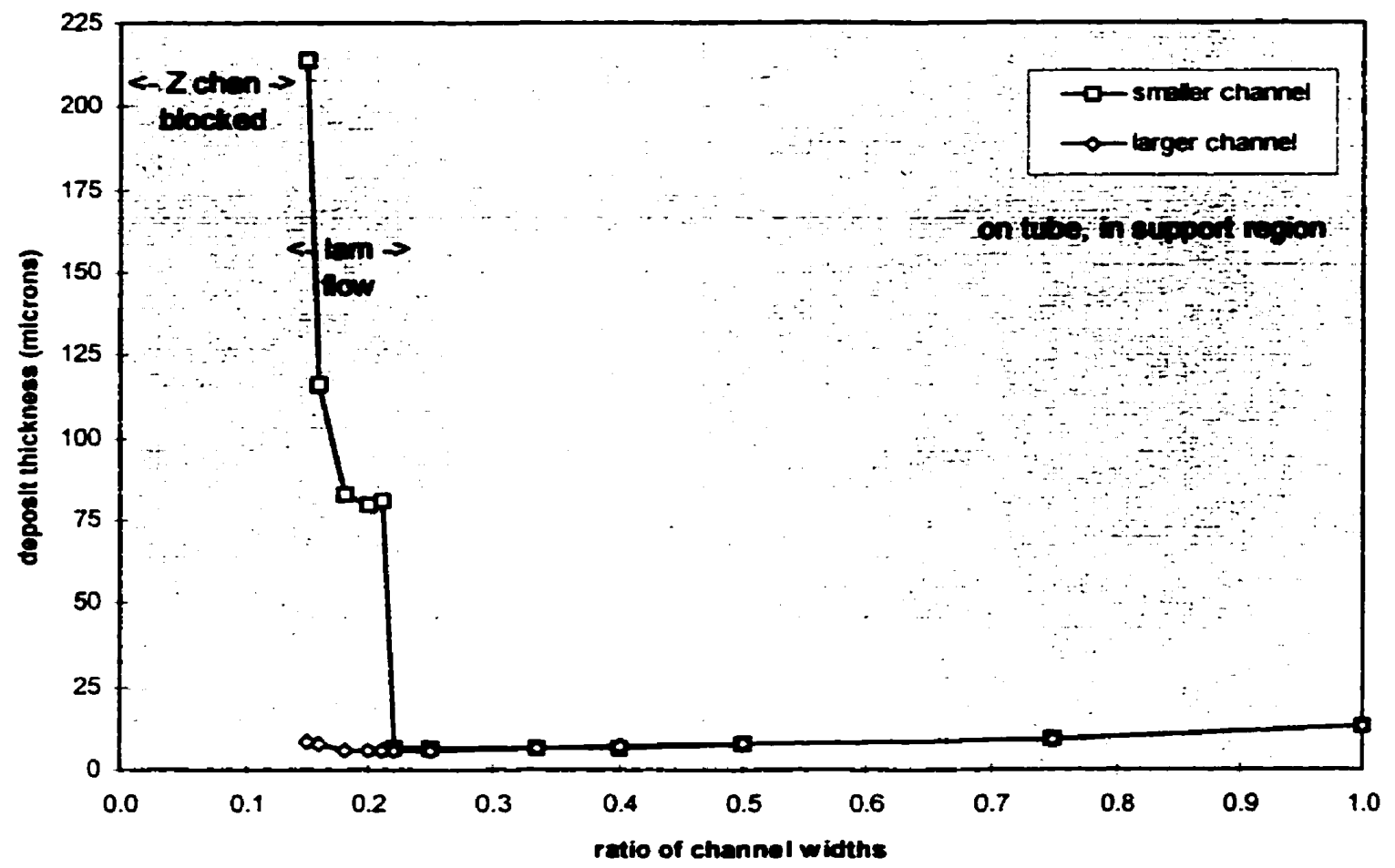

Input values:

initial Z-channel annular gap width initial Z-channel rect. half-width initial mass flux

flag for unequal channel sizes FCH all other values:

variable, 2.1 to $0.32 \mathrm{~mm}$ variable, 2.1 to $0.32 \mathrm{~mm}$ $80 \mathrm{~kg} / \mathrm{m}^{2} . \mathrm{s}$ 1 reference values as per Table 10.2 
Figure 10.9: Prediction for bifoil fouling experiment

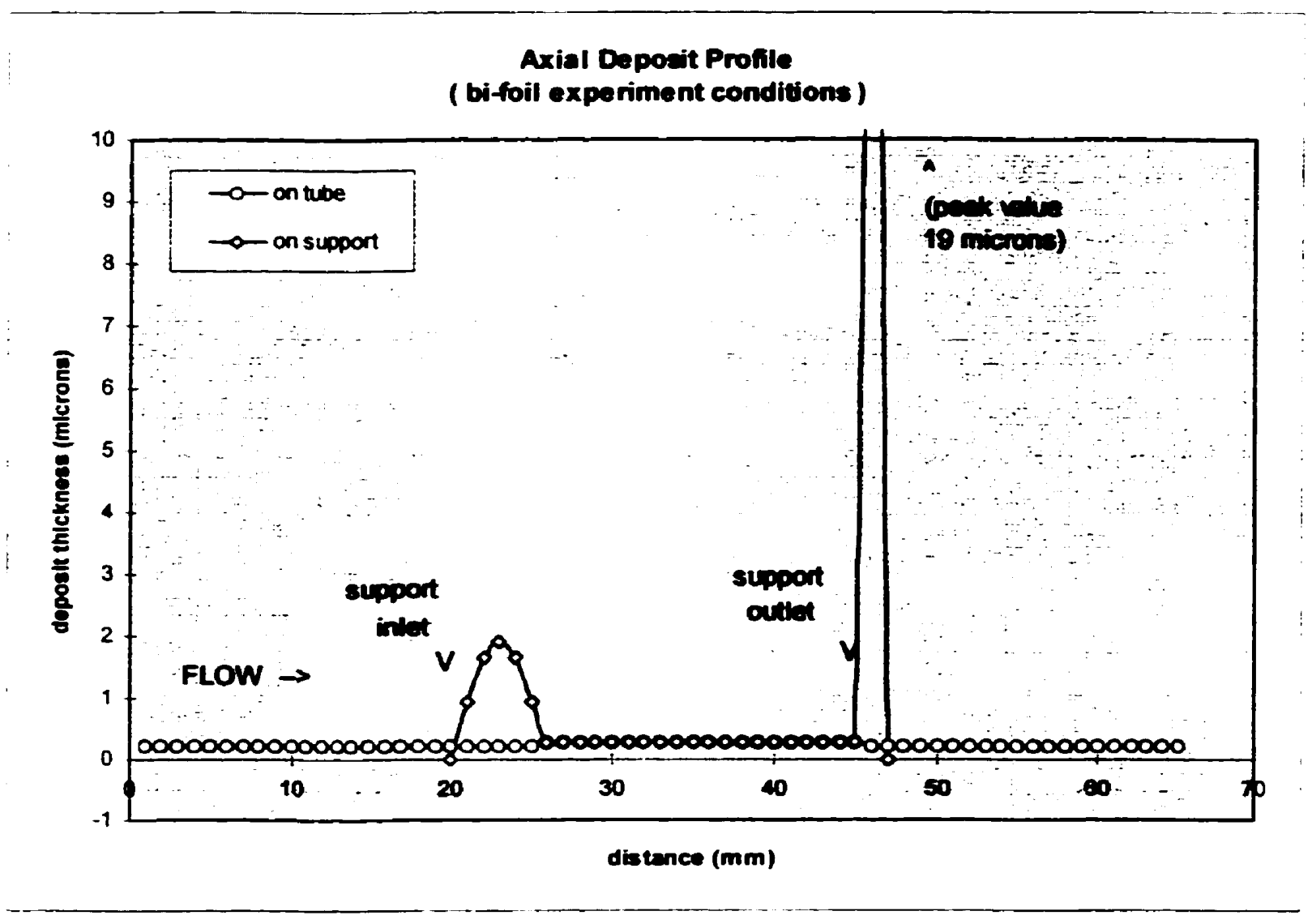

Input values:

see Table 10.4 
Figure 10.10: Long-term prediction for Bruce A SG

(a) reference conditions with all models on

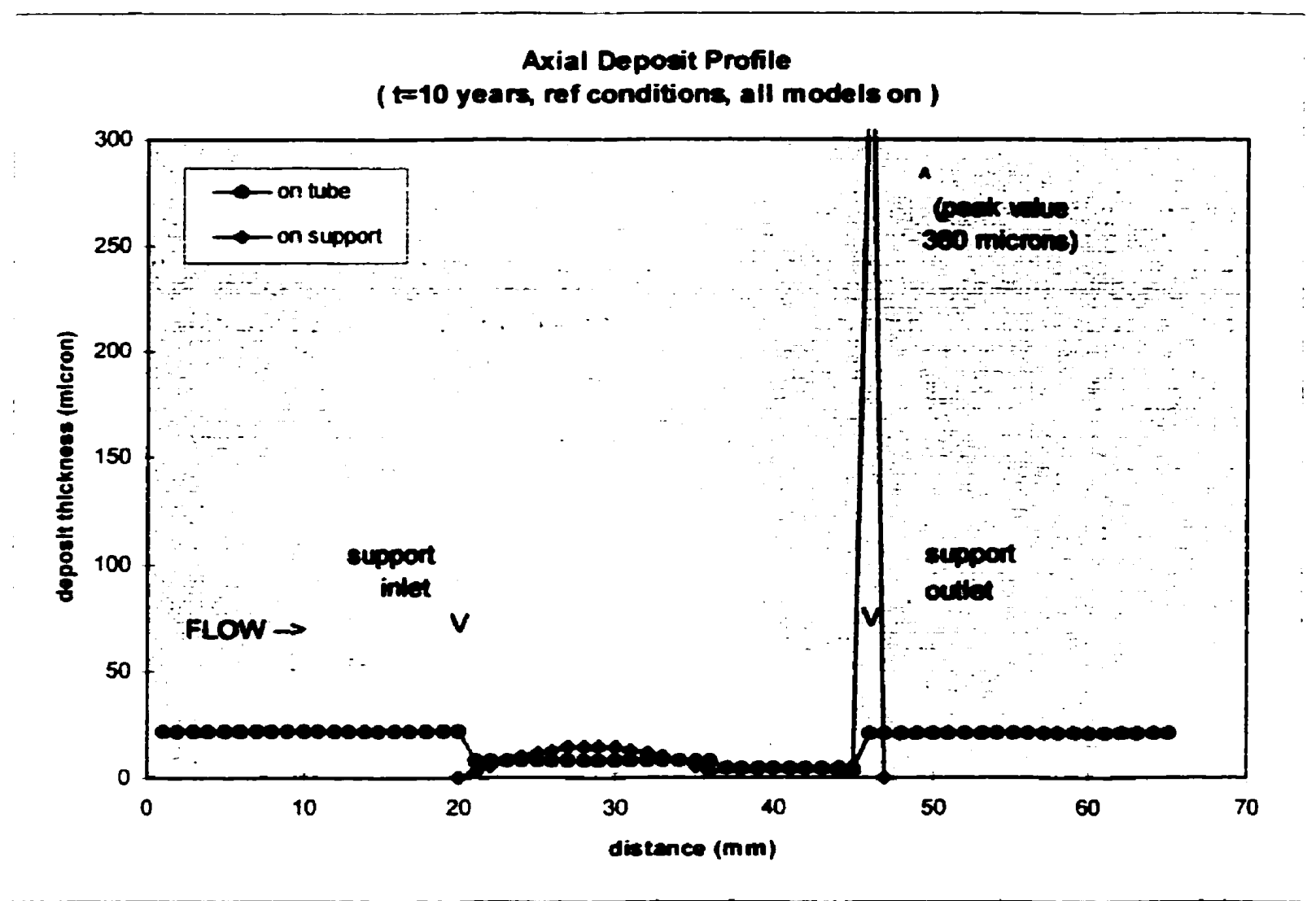

Input values:

time period

flag for particle impaction FKF

flag for settling in vena region FKV

flag for settling on top of support FKS

flag for consolidation FCO

flag for flow development FFD

flag for unequal channel sizes FCH

all other values:

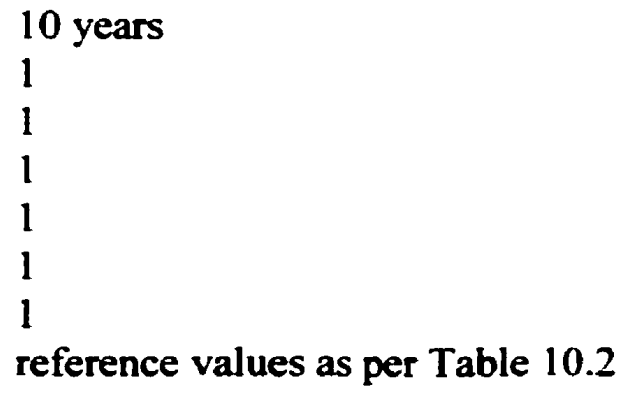


Figure 10.10 (continued)

(b) with less suppression

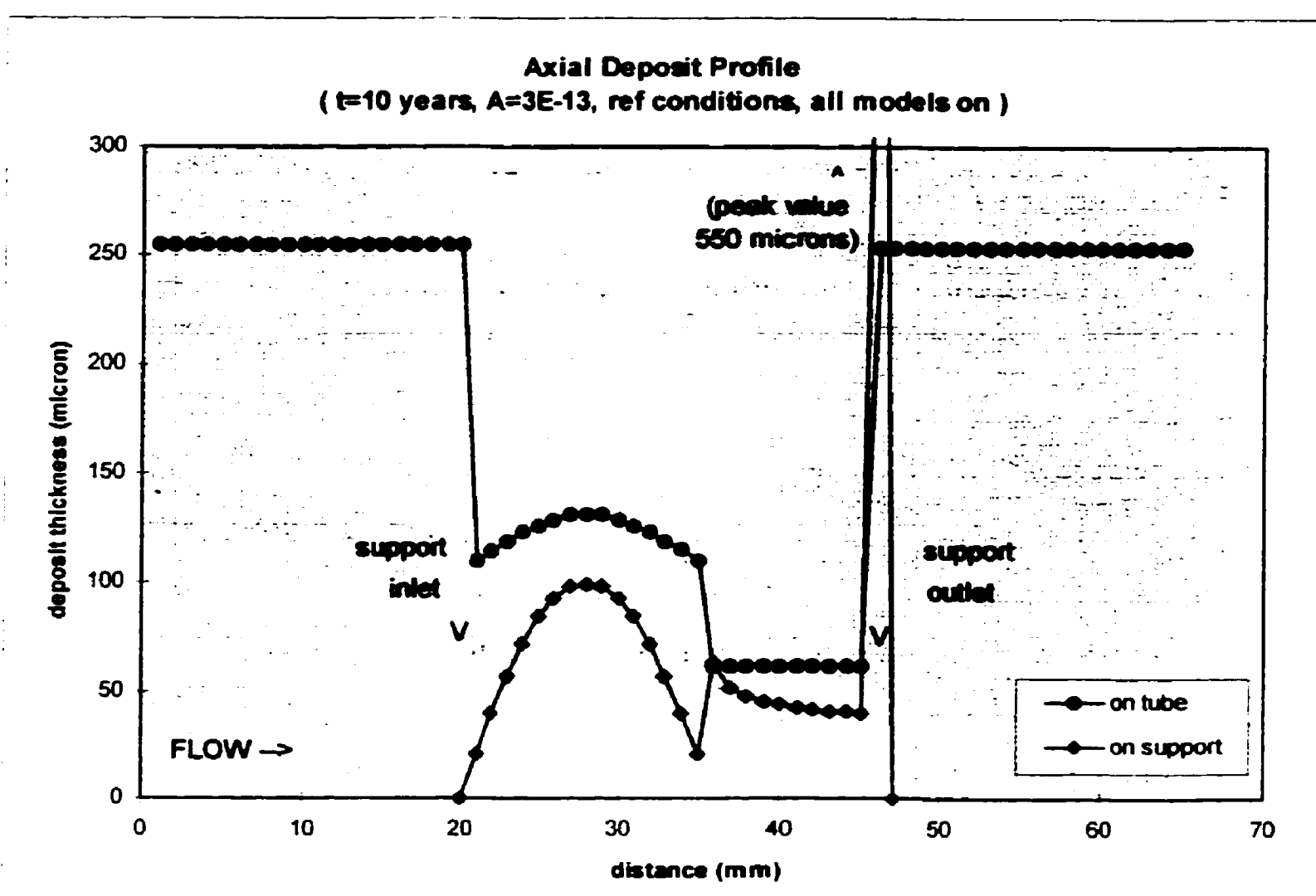

Input values:

time period

suppression factor $A$

flag for particle impaction FKF

flag for settling in vena region FKV

flag for settling on top of support FKS

flag for consolidation FCO

flag for flow development FFD

flag for unequal channel sizes $\mathrm{FCH}$

all other values:

10 years

$3 \times 10^{-13}$

1

1

1

1

1

1

reference values as per Table 10.2 
Figure 10.10 (continued)

(c) effect of varying suppression factors on deposit growth

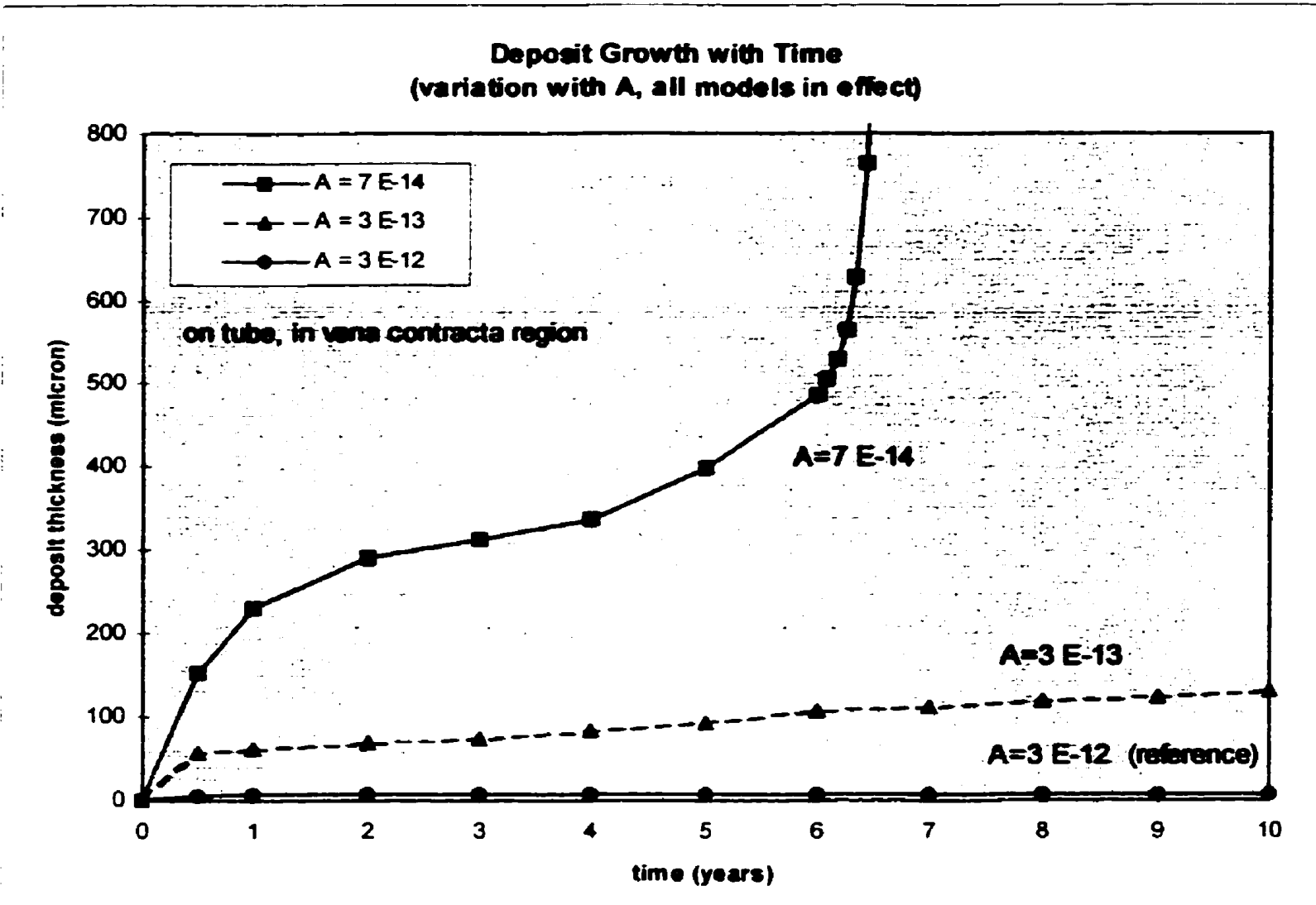

Input values:

time period

suppression factor $A$

flag for particle impaction FKF

flag for settling in vena region FKV

flag for settling on top of support FKS

flag for consolidation FCO

flag for flow development FFD

flag for unequal channel sizes FCH

all other values:

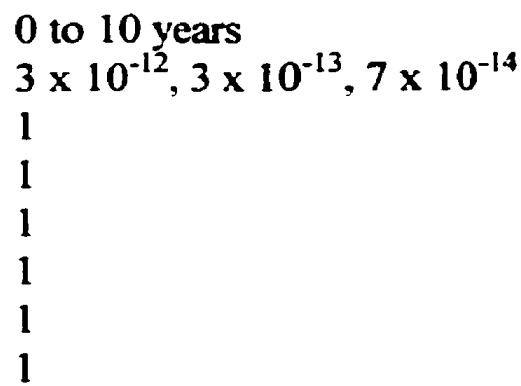

reference values as per Table 10.2 
Figure 10.10 (continued)

(c) with unequally-sized parallel channels in support, Y channel

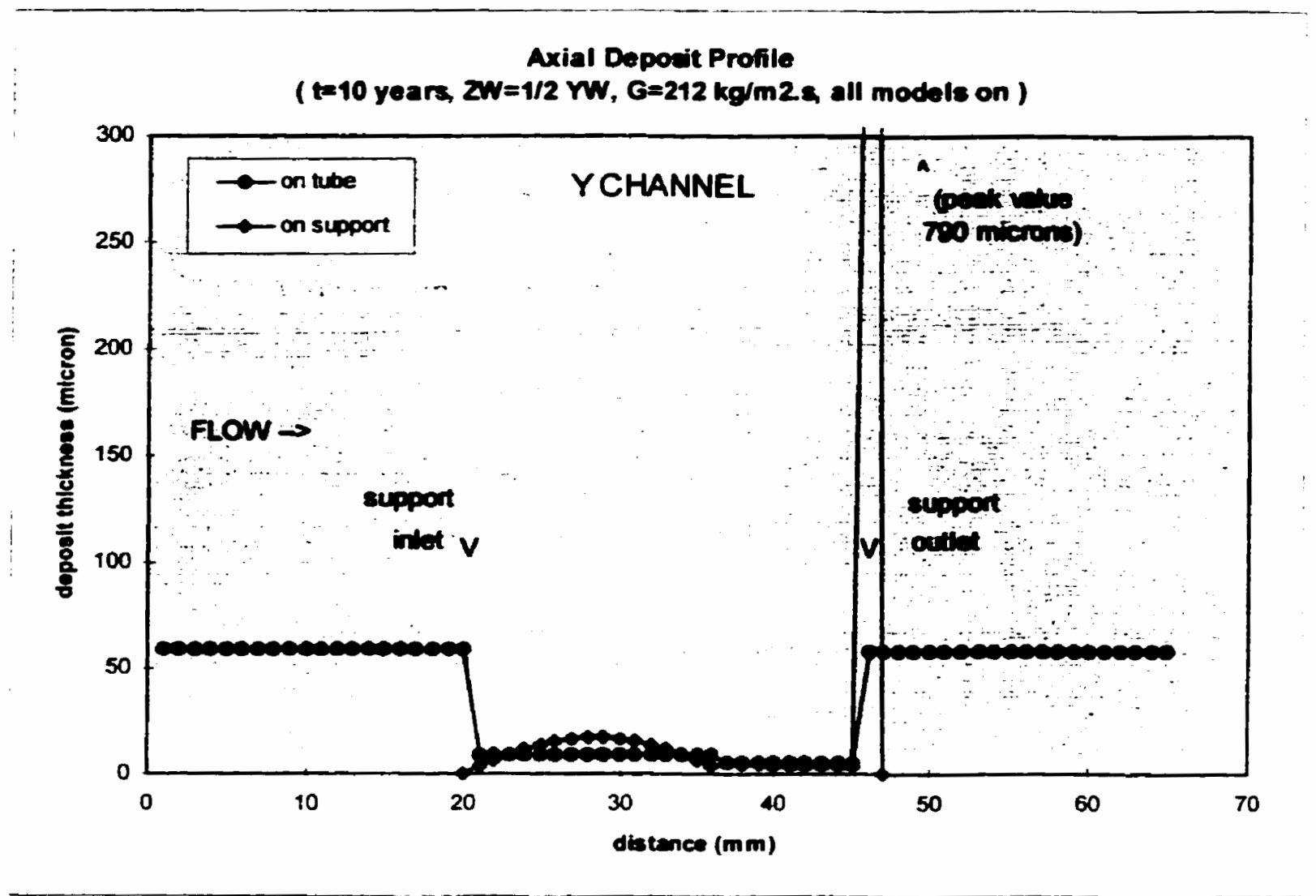

Input values:

time period

initial Z-channel annular gap width initial Z-channel rect. half-width initial inlet mass flux

flag for particle impaction FKF

flag for settling in vena region FKV

flag for settling on top of support FKS

flag for consolidation FCO

flag for flow development FFD

flag for unequal channel sizes FCH

all other values:

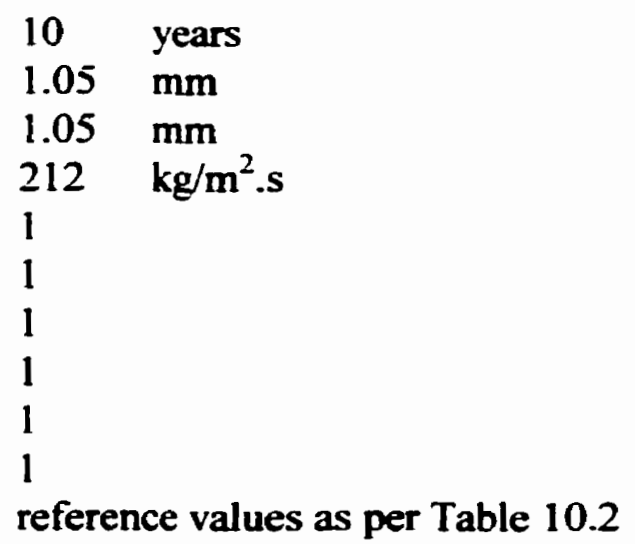


Figure 10.10 (continued)

(d) with unequally-sized parallel channels in support, $Z$ channel

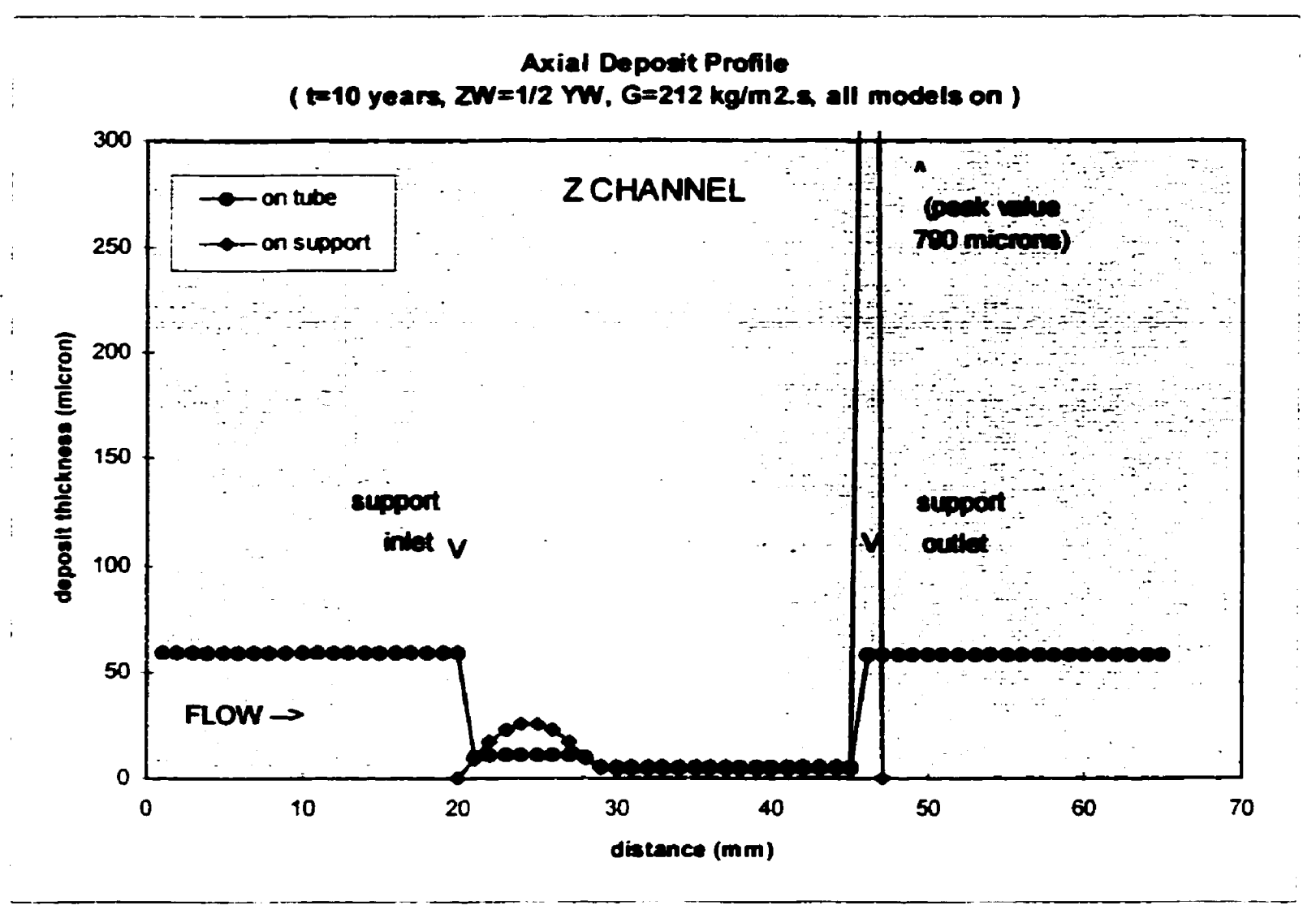

Input values:

same as on previous page 


\section{Chapter 11}

\section{SUMMARY AND RECOMMENDATIONS}

As fouling of supports has begun to limit the operation of some SGs, there is a need to understand and assess the fouling propensity of various support designs. Particle deposition on SG tubes and supports is clearly a complex phenomenon involving many mechanisms that interact. The approach taken in this work was to (1) characterize the two-phase flow patterns of existing designs, (3) correlate these with fouling patterns observed in the field, and (2) develop some support fouling models to better understand the dynamics of the fouling mechanisms.

\subsection{Summary of Experimental Observations}

In the flow characterization experiments, the following flow features were observed near support mockups:

- undisturbed flow upstream of the support,

- a vena-contracta effect at the entrance to the support subchannel, which was most pronounced with the sharp-edged and more obstructive supports,

- high concentration of vapour in these vena contracta regions, due to flashing of liquid to vapour and/or the collection of vapour in a small recirculation zone,

- significant turbulence downstream of the support (wake region),

- for supports with large surface areas normal to the flow (e.g., broached plates): dramatic recirculation of fluid downstream at low quality. leading to semi-stagnant vapour pockets at higher qualities,

- for supports with narrow surfaces (e.g., Wolsung-1 formed bars): no recirculation but small vapour pockets directly over the support, 
- for symmetric supports (e.g., broached plates): stable flow patterns featuring distinct high- and low-velocity regions.

- for non-symmetric supports (e.g., lattice bars): greater mixing and cross-flow.

\subsection{Summary of Correlation with Fouling Patterns}

These flow patterns have helped to explain some fouling trends in SGs currently in operation. In particular:

- "Lipping" at the inlet edges of broach holes are likely related to the presence of a vena contracta region where the boundary layer separates from the wall following a sharp contraction. The low-velocity zone encourages particle trapping. The growing deposit is likely to be consolidated by precipitation of cementing agents (iron, copper), by flashing due to sudden pressure loss. [However, flashing would be less pronounced under SG pressures than it was in the test loops.] Low-velocity zones on the downstream surface of the support can sustain a deposit in a similar manner and may even cause lipping at the outlet.

- Thick, coarse deposits on the underside of especially the $1^{\text {st }}$ support are likely related to the impaction and trapping of particles and agglomerates in the SG feedwater or kicked up from the tube sheet. Gravitational and/or centrifugal settling could account for thinner, finer deposits on the topside.

- Deposition driven by thermal action (e.g., lipping and consolidation) can be expected to be most pronounced where the combination of mass flux and quality (and hence the two-phase pressure loss) is highest, i.e., at the top of the SG for units with no internal preheater, and near the middle for units with internal preheater, and on the hot leg rather than the cold leg. 


\subsection{Summary of Fouling Susceptibility Criteria}

A fouling-resistant design will have:

- low flow resistance and streamlined edges (especially on the upstream side),

- low potential for particle trapping upstream,

- low potential for particle trapping downstream,

- uniformity in subchannel size to discourage preferential fouling in one size of subchannel, and

- design asymmetry that favours flow mixing.

\subsection{Summary of Predictions using TSFOUL}

The use of TSFOUL has helped to understand the complex dynamics of deposition in the CANDU SGs. In particular:

- In the free-span region, the dominant deposition mechanism is boiling (on the tube). In the support region, the transport mechanisms tend to dominate. However, in turbulent flow, a suppression effect based on a factor of $\mathrm{A}=3 \times 10^{-12}$ generally supersedes both these effects.

- As expected, deposit thickness decreases as mass flux, quality, or suppression factor increases. The thickness increases as particle size or concentration increases.

- Particle impaction on the support bottom would occur under SG conditions if the particle size were relatively large (e.g., $>8 \mu \mathrm{m})$.

- Specifying a single particle diameter or concentration is limiting, as SGs carry particles of varying sizes and correspondingly varying concentrations. 
- Gravitational and centrifugal settling appear to be sensible models for predicting build-up on the top of the support and at the vena contracta eddy.

- The consolidation model would be an meaningful way of accounting for enhanced deposition at the support inlet, except that its effect is dominated by the current suppression model.

- Accounting for developing flow increases the deposition on the support, but only modestly so.

- Among unequal parallel channels in the support, the narrower channel tends to have the lower void fraction and the greater deposit thicknesses.

- As the smaller channel (in an unequal parallel set) becomes narrower, its deposit thicknesses reach a limit beyond which no further growth occurs, due to the diminishing of the particle supply to that channel. If this channel experiences transition to laminar flow, the deposits are predicted to grow rapidly in spite of the reduced supply, possibly to the point of blockage.

- A comparison with a laboratory fouling test shows that the deposition is underpredicted, even with the use of the support-specific models. More experimental data are clearly needed for model fine-tuning and validation.

- Long-term (10 year) predictions show modest deposition, except when the suppression factor $A$ is reduced. This shows that the suppression term is very dominant, and predictions rely heavily on establishing the appropriate A value. 
- At a value of $A=7 \times 10^{-14}$, deposits were predicted to occupy most of the support channel after $\sim 10$ years, at which point convergence difficulties occurred that were reminiscent of flow instability.

- The long-term predictions show that a narrower channel will eventually have a thicker deposit on its support than its wider neighbor.

- The critical region for fouling and blockage appears to be the vena contracta region, on both the tube and the support surfaces.

- The overall uncertainty of the simulations is dictated by that of the suppression factor A and that of the particle size assumption, and is difficult to establish. However, the simulations are judged to provide better than order-of-magnitude agreement.

\subsection{Tools Developed to Predict Fouling}

The work reported here has generated some experimental and analytical tools that can be used in a qualitative manner to design fouling-resistant supports, or to evaluate the fouling susceptibility of existing ones:

- Fouling resistance criteria for supports (listed in Table 6.1) help to evaluate specific support designs.

- The proposed fouling mechanisms (Figure 6.1) help to understand observed fouling distributions and morphologies.

- The distribution of deposits across the SG can be anticipated by considering the mass flux and quality distribution (Section 6.2), because a combination of high mass flux and high quality contributes to high pressure loss and hence fouling propensity. 
- Experimental tools such as simple flow visualization tests using air bubbles in water (Sections 4.3 and 5.3) and measurement of axial pressure loss profiles (Sections 4.4 and 5.4) help to characterize the extent of flow disruption by the support. A comparison can then be made with existing designs whose fouling propensity has been reasonably well documented, e.g., the broached plates.

- Analytical tools such as the TSFOUL program (Chapters 7 to 10) help to understand and predict the distribution and growth rates of deposits on tubes and supports.

\subsection{Recommendations}

Once data become available from support fouling experiments currently underway at CRL, the support-specific models can be validated and fine-tuned. Validation data for prediction tools such as TSFOUL and SLUDGE are sorely needed. Validation is important in establishing the credibility of models, but it is limited for programs such as TSFOUL or SLUDGE by the challenge of acquiring data under actual SG conditions, and by the complexity of the interactions of the mechanisms involved.

Some work is still required to improve the support-specific models in TSFOUL, as stated at the end of each section in Chapter 9. TSFOUL also needs to be made more widely applicable: it is currently set up for the broached-plate geometries only, and needs to be applied to other support geometries such as the lattice bars. Once such improvements are made, the TSFOUL program could be used for routine SG fouling predictions as follows:

Option \#1: The program could run alongside a code such as SLUDGE, taking in the same conditions of flow, quality, etc. at a given time $t$. (The program would be the equivalent of a subroutine.) It would then predict deposit thicknesses for the support, then feed this information back to SLUDGE. The support geometry would need to be input into TSFOUL prior to use. 
Option \#2: The program could be run separate from the large code to determine a fouling "enhancement" factor for the specific SG operating conditions and support design of interest. This factor is then input into the code, similar to a pressure loss $\mathrm{K}$-factor or a two-phase multiplier. This is the more practical approach. 
APPENDICES 


\section{APPENDIX A: BASIC DEPOSITION MODELS}

\section{A.1 General Description of SLUDGE Code}

The SLUDGE numerical code [1] was developed at CRL to calculate the deposition of particles in a CANDU nuclear steam generator. The local thermalhydraulic conditions, calculated first by another code (called THIRST [2]), are fed to the SLUDGE code.

SLUDGE then calculates the magnetite particle concentration at various locations in the steam generator and the extent of deposit buildup on the tubes, supports, and tube sheet. The supports are modeled simply as volumes of reduced flow area (i.e., lower porosity).

This Appendix describes the deposition equations used in the original SLUDGE code [1], as well as refinements later implemented into the code by its authors.

\section{A.2 Governing Equations}

The growth of a deposit was first formulated by Kern \& Seaton [3] as separate deposition and removal quantities, combined into a net difference or accumulation equation. For SLUDGE, this was expressed as the mass rate of particle deposition and re-entrainment at a surface. $\mathrm{dm} / \mathrm{dt}$, and is given by

$$
\frac{d m}{d t}=K \rho \phi(t)-\lambda m
$$

The particle deposition coefficient, $K$, is referred to as the deposition velocity as it has units of $\mathrm{m} / \mathrm{s}$. $\mathrm{K}$ is based on various deposition mechanisms, described in Sections A.3 to A.5. Equation $A$. 1 is a differential equation with initial condition $m=0$ at $t=0$. For constant concentration $\phi$, the solution is 


$$
m=m *(1-\exp (-\lambda t))
$$

With the exponential term, the deposition rate in turbulent flow reaches an asymptote with time. The parameter $m^{*}$ is the asymptotic amount of deposit reached after infinite time, given by

$$
m^{*}=\frac{K \phi \rho}{\lambda}
$$

$\lambda$ is a re-entrainment term that, in SLUDGE, is taken from Cleaver \& Yates' analysis of simultaneous deposition and re-entrainment of particles at a surface [4]. $\lambda$ is a function of a non-dimensional constant $A$, the friction velocity $u^{*}$, and the liquid kinematic viscosity:

$$
\lambda=\frac{A\left(u^{\cdot}\right)^{2}}{v_{f}}
$$

The friction velocity $u^{*}$ is a function of the shear stress and hence the Darcy friction factor $f$ :

$$
\text { where } \quad u^{\cdot}=u_{f} \sqrt{\frac{f}{8}}
$$

The constant $\mathbf{A}$ is determined experimentally from measured rates of particle removal. Based on deposition experiments under low-temperature, low-pressure conditions, Turner et al. [5] initially reported a value of $A=7 \times 10^{-11}$. [This value was subsequently found to overemphasize deposit removal in the SG and the constant $A$ is now believed to approach zero. i.e., very little removal in effect [6].]

Substituting for $\lambda$ in Equation A.3, the deposited mass per unit area is 


$$
m=K \phi \rho \frac{v_{f}}{A\left(u^{*}\right)^{2}}\left[1-\exp \left(\frac{-A t\left(u^{\circ}\right)^{2}}{v_{f}}\right)\right]
$$

The deposit thickness $\delta$ is obtained by dividing $m$ by the deposit density and porosity*:

$$
\delta=\frac{m}{\rho_{s}\left(1-\beta_{s}\right)}
$$

\section{A.3 Deposition Coefficient $K$}

\section{A.3.1 Single-Phase Deposition Coefficient}

Following Bowen \& Epstein [7], and Ruckenstein \& Prieve [8], the expression for the overall single-phase deposition coefficient, $K_{1 \phi}$. consists of the transport $\left(K_{T}\right)$ and attachment $\left(K_{A}\right)$ coefficients working as two steps in series:

$$
K_{\mathrm{l} \bullet}=\left[\frac{1}{K_{T}}+\frac{1}{K_{s}}\right]^{-1}
$$

For vertical surfaces, the transport coefficient, $\mathrm{K}_{\mathrm{T}}$, is the sum of contributions from thermophoresis, diffusion, and inertial coasting which act in parallel (and are described in more detail in Section A.4):

$$
K_{T}(\text { vertical })=K_{T H}+K_{D}+K_{I}
$$

The $K_{T}$ value for horizontal surfaces includes all of the contributions in Equation A.9, plus additional contributions from gravitational and centrifugal settling: 


$$
K_{T}(\text { horizontal })=K_{T H}+K_{D}+K_{I}+K_{G}+K_{C}
$$

\section{A.3.2 Two-Phase Deposition Coefficient}

The expression for the overall two-phase deposition coefficient, $K_{2 \phi}$, is the sum of the singlephase $\left(K_{1 \phi}\right)$ and a boiling $\left(K_{B}\right)$ coefficient. This is different from the model in the original SLUDGE code [1], and is based on recent findings by Tumer \& Godin [9] in which boiling was found to enhance deposition in a direct manner:

$$
K_{2 \varphi}=K_{1 \varphi}+K_{B}
$$

When liquid is vaporized at a heated surface, the particles and/or solubles that the liquid carried to the surface are left behind near the bubble nucleation site. This is the source of deposition by boiling. The expression for the boiling deposition coefficient $K_{B}$ was obtained from Asakura et al. [10]:

$$
K_{B}=\frac{B Q}{h_{\sqrt{g}} \rho}
$$

where $B$ is a constant determined experimentally [ 1 ].

\section{A.4 Models for Particle Transport}

\section{A.4.1 Thermophoresis}

Thermophoresis is the tendency for colloidal particles $(<0.5 \mu \mathrm{m}$ in diameter) to migrate away from hot walls when there is a temperature gradient. This is due to the hot region being more agitated (e.g., more particle collisions), which drives the particles toward the less agitated cool region. SLUDGE uses the expression for the thermophoresis deposition coefficient $K_{T H}$ 
determined by McNab \& Meisen [11], divided by 6 to bring it into line with experimental loop data [12].

$$
K_{T H}=\frac{l}{6} \frac{-0.26 Q v_{f}}{\left(2 k_{f}+k_{s}\right)\left(T_{b}+273.15\right)}
$$

The negative sign represents a net transport of particles away from a heated wall such as the SG tube. $\mathrm{K}_{\mathrm{TH}}$ tends to be insignificant under the SG conditions of interest because of the relatively small temperature differences between heated surface and bulk fluid.

\section{A.4.2 Inertial coasting}

In the inertia regime, the particles are sufficiently large and have sufficient momentum that turbulent eddies give some of them a "free flight" toward the wall and through the viscous sublayer. The expression for the inertial coasting deposition coefficient $K_{I}$ used in SLUDGE is originally from aerosol work done by Cleaver \& Yates [13], with the magnitude reduced by a factor of 100 to bring it into agreement with Burrill's deposition data for $1 \mu \mathrm{m}$ particles of magnetite in the inertial coasting regime [14]:

$$
K_{l}=\frac{1}{100} \frac{1}{5.23} u i_{p} \frac{\rho}{\rho_{s}} \exp \left(0.48 t_{p}^{-}\right)
$$

where the non-dimensional relaxation time $t_{p}{ }^{+}$is:

$$
t_{p}=\frac{1}{18} \frac{\rho_{s}}{\rho}\left(\frac{u D_{s}}{v_{f}}\right)^{2}
$$

Burrill's work [14] indicated that the inertial coasting regime did not extend beyond $\operatorname{Re}=50,000$. Turner [6] showed that this limit corresponded to $t_{p}{ }^{+} \approx 0.1$ to 0.2 . 


\section{A.4.3 Diffusion}

In the diffusion regime, suspended particles move with the fluid and are carried to the wall by the Brownian motion of the fluid molecules and eddies. The eddy and molecular diffusion deposition coefficient, $\mathrm{K}_{\mathrm{D}}$, for turbulent flow is based on an expression by Cleaver \& Yates [13], updated in Turner \& Godin [9] to better suit SG turbulent-flow conditions $(\operatorname{Re}>3000)$ :

$$
K_{D_{\text {, surt }}}=0.031 \cdot\left(\frac{D}{v_{f}}\right)^{2 / 3}
$$

where $\mathrm{D}$ is the diffusivity described by:

$$
D=\frac{k\left(T_{b}+273.15\right)}{3 \pi \rho v_{f} D_{s}}
$$

and $\mathrm{k}$ is the Boltzmann constant $\left(=1.3805 \times 10^{-23} \mathrm{~kg} \mathrm{~m}^{2} \mathrm{~K}^{-1} \mathrm{~s}^{-2}\right)$. Note that, in SLUDGE, laminar flow is not considered from the standpoint of deposition.

\section{A.4.4 Gravitational and Centrifugal Settling}

For sufficiently large and/or heavy particles near non-vertical surfaces, gravity may control the deposition process. The expression for the gravitational settling deposition coefficient $\mathbf{K}_{\mathbf{G}}$ is based on Stokes' Law, which gives the rate of fall (or terminal velocity) of a small sphere in a viscous fluid.

$$
K_{G}=\frac{\left(\rho_{s}-\rho\right) g D_{s}^{2}}{18 \rho v_{f}}
$$


In regions where the fluid changes direction quickly, such particles may be subject to centrifugal forces that propel the particles toward the wall. The expression for the centrifugal settling coefficient $\mathrm{K}_{C}$ is similar to that of gravitational settling. It is based on Stokes' law for a spherical particle in a centrifugal field [15]. Instead of being subject to gravitational acceleration, represented by " $\mathrm{g}$ ", the particle is accelerated by the flow, represented by the velocity squared times the fluid curvature $k_{c}$ (defined as $1 / R$ where $R$ is the radius of the streamline curvature). The terminal centrifugal settling velocity is given by:

$$
K_{C}=\frac{\left(\rho_{s}-\rho\right) u_{f}^{2} k_{c} D_{s}^{2}}{18 \rho v_{f}}
$$

Note that, in SLUDGE, $K_{G}$ and $K_{C}$ are applied to horizontal surfaces only.

\section{A.5 Model for Particle Attachment}

The expression for the attachment coefficient $\mathrm{K}_{\mathrm{A}}$ is based on experimental results by Turner \& Godin [9] on the rate of attachment of magnetite to Inconel-600 under SG conditions and in turbulent alkaline water. This rate was found to be 500 times lower than the rate derived from low-temperature data [12], implying less attachment at higher temperatures. The rate of magnetite deposition onto I-600 was found to be attachment-limited rather than transportlimited, due to repulsive forces between similarly-charged particles and surface.

Turner's expressions for $K_{A}$ include an activation energy term. The best fit to his hightemperature data [9] gives (with $K_{0}=5.697, E_{a}=76.4 \mathrm{~kJ} / \mathrm{mol}$, and $T_{w}$ having units of $K$ ):

$$
K_{d}=K_{o} \exp \left(\frac{-E_{a}}{R T_{w}}\right)=\exp \left(1.74-\frac{9187}{T_{w}}\right)
$$




\section{References}

[1] Y. Liner, M.B. Carver, C.W. Turner, and A.O. Campagna, "Simulation of Magnetite Particulate Fouling in Nuclear Steam Generators", Proceedings of the ASME meeting on Steam Generator Sludge Deposition in Recirculating and Once Through Steam Generator Upper Tube Bundle and Support Plates, NE-Vol.8, Book No. G00690, 1992.

[2] M.B. Carver, L.N. Carlucci, and W.W.R. Inch, "Thermal-hydraulics in Recirculating Steam Generators. THIRST Code User's Manual", AECL Report No. AECL-7254, 1981 .

[3] D.Q. Kern and R.E. Seaton, "A Theoretical Analysis of Thermal Surface Fouling", British Chemical Engineering, pp.258-262 (1959 May).

[4] J.W. Cleaver and B. Yates. "The Effect of Re-Entrainment on Particle Deposition". Chemical Engineering Science, Vol.31, pp.147-151 (1976).

[5] C.W. Turner, D.H. Lister, and D.W. Smith, "The Deposition and Removal of SubMicron Particles of Magnetite at the Surface of Alloy 800", Proceedings of the $1^{\text {st }}$ Steam Generator and Heat Exchanger Conference (sponsored by the Canadian Nuclear Society), 1990 April 30 to May 2, Toronto, Canada.

[6] C.W. Turner, "Rates of Particle Deposition from Aqueous Suspensions in Turbulent Flow: A Comparison of Theory with Experiment", Chemical Engineering Science, Vol.48, No.12, pp.2189-2195 (1993).

[7] B.D. Bowen and N. Epstein. "Fine Particle Deposition in Smooth Parallel-Plate Channels", Journal. of Colloid Interface Science, Vol.72, pp.88-97 (1979). 
[8] E. Ruckenstein and D.C. Prieve, "Rate of Deposition of Brownian Particles Under the Action of London and Double-Layer Forces", Journal of Chemical Society Far. Transactions, II, Vol. 69, pp. 1522-1536 (1973).

[9] C.W. Turner and M. Godin, “Mechanisms of Magnetite Deposition in Pressurized Boiling and Non-Boiling Water", Proceedings of the 2nd Int. Conf. on Steam Generators \& Heat Exchangers, Toronto, Canada, 1994 June 13-15.

[10] Y. Asakura, M. Kikuchi, S. Uchida, and H. Yusa, "Deposition of Iron Oxide on Heated Surfaces in Boiling Water", Nuclear Science \& Engineering, Vol.67, pp.1-7. (1978).

[11] G.S. McNab and A. Meisen, "Thermophoresis in Liquids", Journal of Colloid Interface Science, Vol.44, pp.339-346 (1973).

[12] C.W. Tumer and D.W. Smith. "A Study of Magnetite Particle Deposition onto Alloy800 and Alloy-600 between 25 and $85^{\circ} \mathrm{C}$ and Predicted Rates under SG Operating Conditions", Proceedings of the ASME meeting on Steam Generator Sludge Deposition in Recirculating and Once Through Steam Generator Upper Tube Bundle and Support Plates, NE-Vol.8, Book No. G00690, p.9, 1992. Also available as Report Nos.AECL-10754 and COG-92-344.

[13] J.W. Cleaver and B. Yates, "A Sub-Layer Model for the Deposition of Particles from a Turbulent Flow", Chemical Engineering Science, Vol.30, pp.983-992 (1975).

[14] K.A. Burrill, "The Deposition of Magnetite Particles from High Velocity Water onto Isothermal Tubes", AECL Report No. AECL-5308, 1977.

[15] C.E. Lapple, Fluid and Particle Mechanics, Edwards Brothers, Inc., Michigan, 1956. 


\section{APPENDIX B: COMPARISON OF PRESSURE LOSS MODELS}

The pressure loss calculations in TSFOUL (described in Section 7.3.5) were verified by comparing their results with those using a model from Idel'chik for thick-edged orifices in a straight channel [1]. The comparison was done for turbulent, single-phase flow.

Idel'chik's model predicts overall pressure loss $\Delta \mathrm{P}$ across the orifice as the sum of four independent losses: sudden contraction, flow development for small $L / D_{\text {hy }}$ orifices, sudden expansion, and friction:

$$
\begin{aligned}
& \Delta P=\frac{\rho u_{\text {sup }}^{2}}{2}\left[K_{\text {con }}+K_{\text {fdev }}+K_{\text {exp }}+K_{f r c}\right], \text { or } \\
& \Delta P=\frac{\rho u_{f s}^{2}}{2}\left(\frac{A_{f s}}{A_{\text {sup }}}\right)^{2}\left[0.5\left(1-\frac{A_{\text {sup }}}{A_{f s}}\right)^{075}+\tau\left(1-\frac{A_{\text {sup }}}{A_{f s}}\right)^{1.375}+\left(1-\frac{A_{\text {sup }}}{A_{f s}}\right)^{2}+f \frac{L}{D_{h y}}\right]
\end{aligned}
$$

where $u_{f s}$ is the velocity in the free-span region $[\mathrm{m} / \mathrm{s}]$,

$A_{i s}$ is the flow area in the (larger) free-span region $\left[\mathrm{m}^{2}\right]$,

$A_{\text {sup }}$ is the flow area in the (smaller) orifice or support region $\left[\mathrm{m}^{2}\right]$, and

$\tau \quad$ is an enhancement factor representing increased pressure loss due to flow development effects [-].

(See Nomenclature at start of thesis for other parameters.)

Flow development, for which values of $\tau$ are tabulated in Idel'chik's handbook [1], is only significant for $L / D_{\text {hy }}<2$. As the $L / D_{\text {hy }}$ is larger than this for the support geometries of interest, the flow development term will be small compared to the overall pressure loss and is therefore neglected here. 
The model outlined in Chapter 7 uses the same approach of summing the terms, and pressure loss values calculated by TSFOUL and those using Idel'chik's model are therefore expected to be similar. The expansion and friction terms are the same as those in Idel'chik's model, but the contraction term is different: for TSFOUL, the contraction coefficients from Perry [2] were adopted, as outlined in Collier [3]. Thus, in TSFOUL,

$$
K_{c o n}(\text { in } T S F O U L)=\left(\frac{1}{C_{c}}-1\right)^{2}
$$

where $C_{c}$ is the coefficient of contraction, which is a function of area ratio and is tabled in Collier [3].

The only other difference is the definition of $u_{\text {sup }}$, i.e., the velocity in the support region. TSFOUL accounts for the vena contracta region, which means the effective flow area reduction is considered greater than the actual reduction. In TSFOUL, $u_{\text {sup }}$ is therefore greater than the corresponding value in Idel 'chik's model, in the vena contracta region. This only affects the contraction term and part of the friction term.

Figure $\mathrm{Bl}$ shows the comparison for a fixed area ratio and varying inlet mass fluxes. Agreement is reasonable (keeping in mind the nature of the log-log scales which magnify low values). Figure B2 is the comparison for a fixed mass flux and varying area ratios, again showing reasonable agreement. These results imply that the pressure loss model has been implemented correctly in TSFOUL.

The following conditions were input for the runs in Figures B1 and B2:

$\begin{array}{lll}\mathrm{P}= & 4.4 & \mathrm{MPa} \\ \mathrm{T}_{\mathrm{b}}= & 250 & { }^{\circ} \mathrm{C} \mathrm{(subcooled} \mathrm{liquid)} \\ \rho= & 799 & \mathrm{~kg} / \mathrm{m}^{3}\end{array}$




$\begin{array}{llll}\mu= & 107 \times 10^{-6} & \mathrm{~kg} / \mathrm{m} . \mathrm{s} & \\ \mathrm{L}= & 0.025 & \mathrm{~m} & \\ \varepsilon= & 2.8 \times 10^{-6} & \mathrm{~m} & \\ \mathrm{D}_{\text {tubc }}= & 0.0191 & \mathrm{~m} & \\ \mathrm{w}_{\mathrm{fs}}= & 0.006 & \mathrm{~m} & \\ \mathrm{w}_{\text {sup }}= & 0.001 \rightarrow 0.005 & \mathrm{~m} & \text { (variable) } \\ \mathrm{G}_{\mathrm{fs}}= & 100 \rightarrow 2000 & \mathrm{~kg} / \mathrm{m}^{2} . \mathrm{s} & \text { (variable) }\end{array}$

\section{References}

[1] I.E. Idel'chik, Handbook of Hydraulic Resistance, $3^{\text {rd }}$ edition, CRC Press Inc., 1994, p. 222.

[2] Perrv's Chemical Engineers' Handbook. $4^{\text {th }}$ edition, McGraw-Hill, 1963, pp.5-30.

[3] J.G. Collier, Convective Boiling and Condensation. 2nd edition, McGraw-Hill Book Company, 1981, p.75. 
Figure B.1: Comparison with Idel'chik model, with varying mass flux

\section{Comparioon with Idel'chik model}

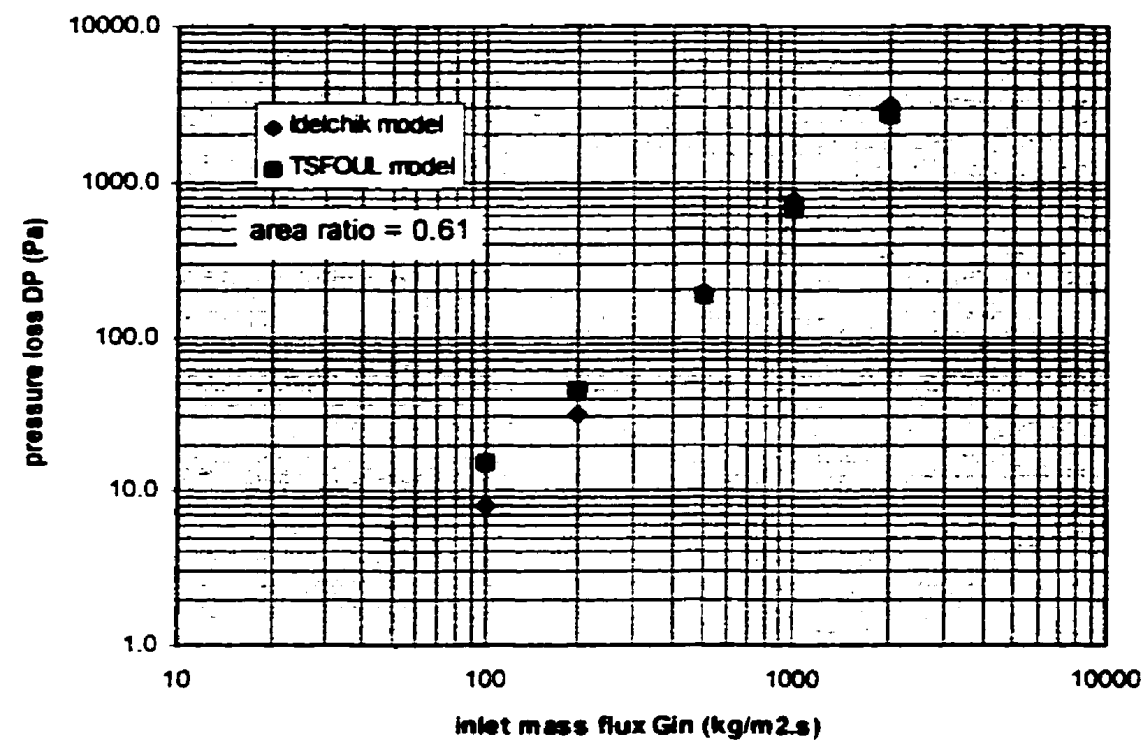

Figure B.2: Comparison with Idel'chik model. with varying flow area ratio

Comparison with Idel'chik model

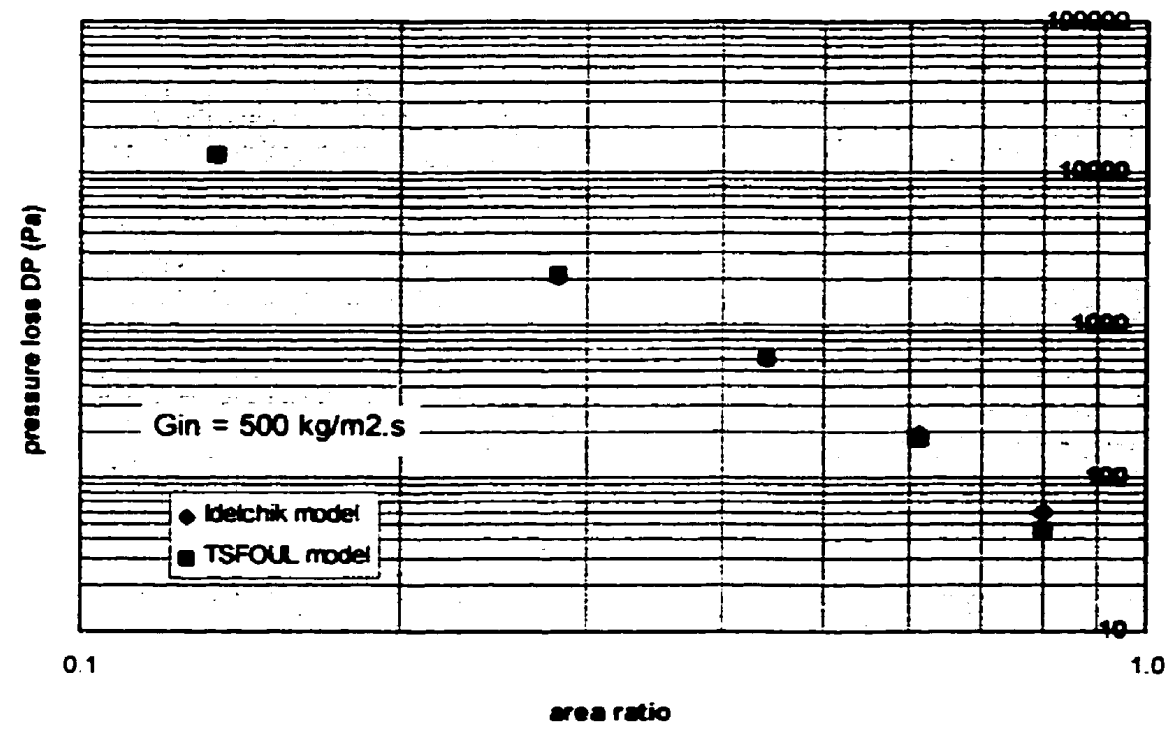




\section{APPENDIX C: LISTING OF TSFOUL PROGRAM}

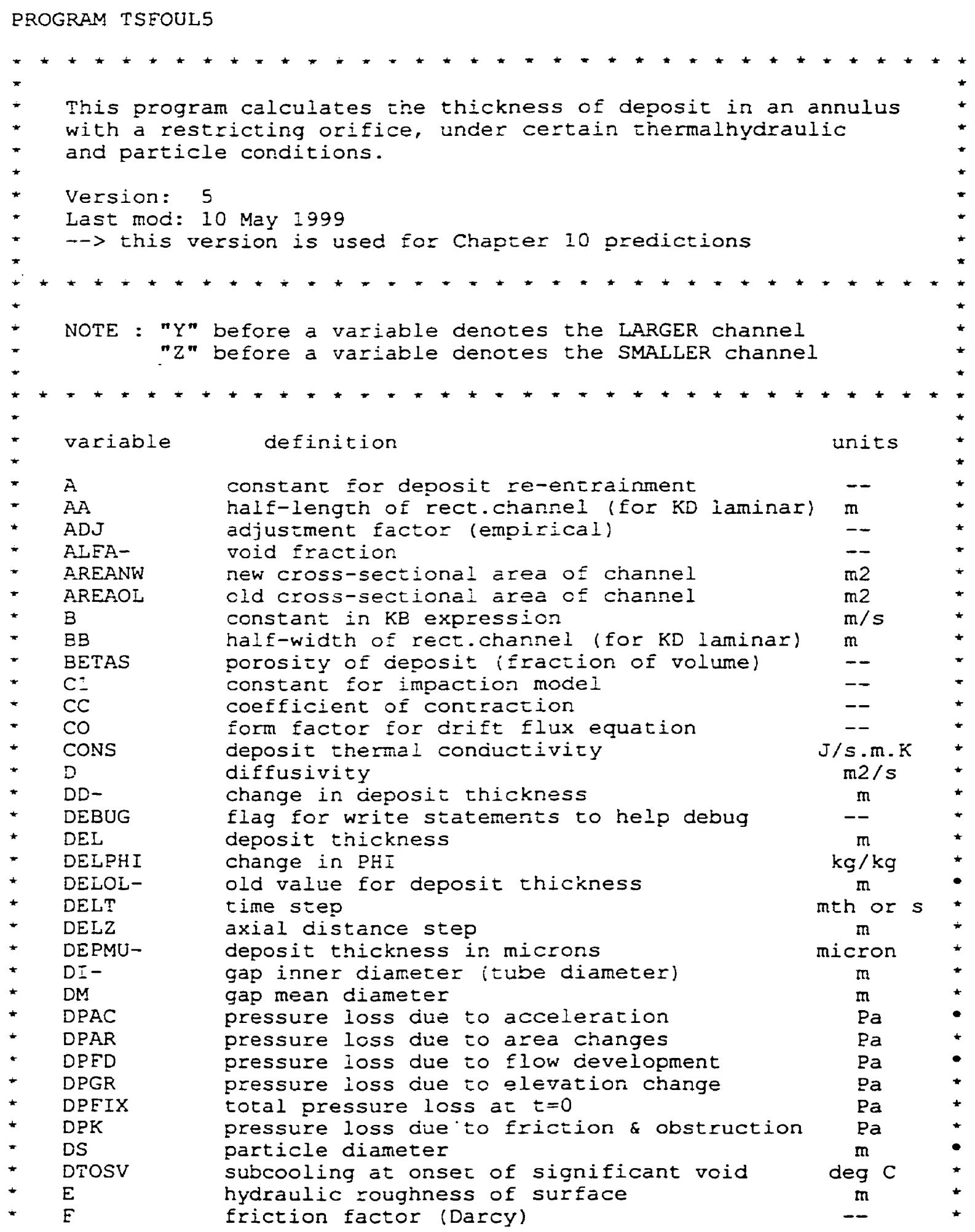


EBO

ECO

ECH

EED

EGK

EKE

EKS

EKV

ELM

EPR

ERAC

ESURE

G-

GG

GSURE

H-

II

JE-, JG-

$\mathrm{K} 1 \mathrm{P}$

K2P

$\mathrm{KA}$

KAREA

$\mathrm{KB}$

$\mathrm{KC}$

KCON

KD

KEXP

$\mathrm{KE}$

KG

$K I$

KK

KSUPP

KF

$\mathrm{KTH}$

K.UBE

KV

KVENA

ID

NIMP

NSTI

N:

$P$

PRES

PE

PHI-

PHIC

PHI2

PHI2E

POROS

PR

$Q$

REF

REEU

REMOVE

RHO

RHOS

$\mathrm{SC}$

SS

T, TS-

TAU flag to turn on heating/boiling off tube

flag to turn on consolidation model

flag to turn on unequal-sized channels

fiag to turn on effect of flow development

flag to turn on effect of ktot on Gin

flag to turr on filtering/impaction

flag to turn on grav/centr settling

flag to turn on centr settl in vena

fiag to turn on laminar flow models

flag to turn on variation of props with $P$

weight fraction of precipitate in deposit

horizontal node length Eor filter model

mass EILX

gravitational acceleration

horizontal node length for settling model

enthalpy of fluid

counter for numier of iterations

Iiquid, gas superficial veiocity

1-phase deposition coefficient

2-phase deposition coefficient

dep.coeff. for attachment

K-factor for area change

dep.coeff. Eor boiling

dep.coeff. for centrifugal settling

K-Eactor for sudden contraction

dep.coeff. for molecular \& eddy diffusion

$\mathrm{K}$-factor for sudder expansion

dep.coeff. For ilitering/impaction

dep.coeff. For gravitation setting

dep.coeff. for inertial coasting

Boltzmann constant

overall dep.coeff. For support surface

dep.coeff. for transport

dep.coeff. for thermophoresis

overall deposition coeff. for tube surface

dep.coeff. for centr.sett1. in vena region

K-factor for area change in vena region

ratio of distance over diameter (for VV)

fraction of particles that impact support

fraction of particles that stick on support

number of time steps for calculation

pressure (for property functions)

pressure (for pressure loss profile)

peclet number ( $=$ Re $\mathrm{Pr}$ )

particle concentration

concentration of soluble cementing agents

two-phase multiplier

Eriction two-phase mult. $x$ friction factor

area ratio betweer: support and free span

Prandt 1 number

heat flux from tube

Reynolds number for two-phase

Reynolds number based on UE

term representing particle removal

fluid mixture density

density of deposit

rate of precipitation of solubles

rate of flow of particulates

bulk, surface temperature

fluid shear stress

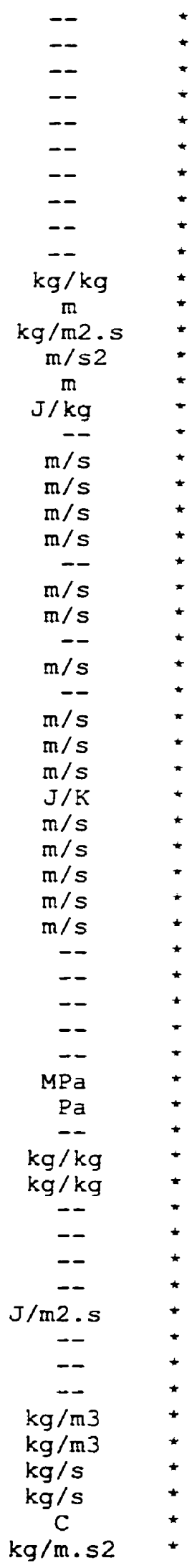




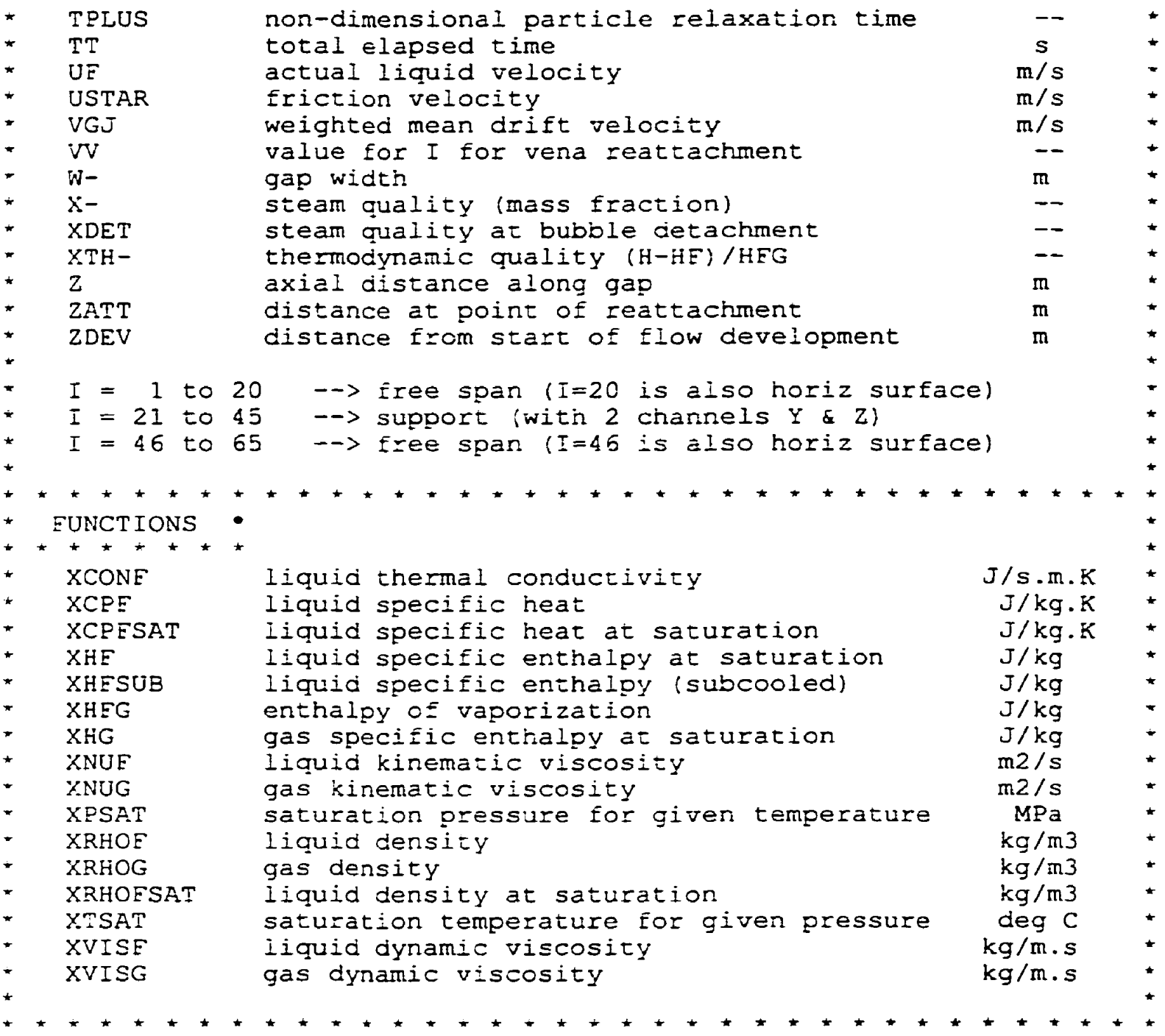

INTEGER I, J,II, VV, YVV, ZVV, NT, DEBUG

INTEGER EBO, EGK, EPR, ELM, EKE, EKV, EKS, FCO, FED, ECH

REAL XTHO, PO, T, Q, GO, TSP, TST, PHIO, DS, DELT

REAL KK, LD, MM, KT, KA, KTUBE, KSUPP, NUE, NUG, KAREA, KEXE

DIMENSION PRES (65)

DIMENSION DI (65),W(65), AREAOL (65), AREANW (65)

DIMENSION RHO (65), G(65), XTH (65), A (65)

DIMENSION DEPMUP (65), DEPMUT (65), DELOLP (65), DELOLT (65)

DIMENSION YDI (45), YW (45), YAREAOL (45), YAREANW (45)

DIMENSION YRHO (45), YG (45), YXTH (45), YA (45)

DIMENSION YDEPMUP (45), YDEPMUT (45), YDELOLP (45), YDELOLT (45)

DIMENSION ZDI (45), ZW (45), ZAREAOL (45), ZAREANW (45)

DIMENSION ZRHO (45), ZG (45), ZXTH (45), ZA (45)

DIMENSION ZDEPMUP (45), ZDEPMUT (45), ZDELOLP (45), ZDELOLT (45)

DOUBLE PRECISION PRES, PSAT, HEPREV, HE 
COMMON /INPT/ T, TSP, TST, Q, DS, CONS, RHOS, E, DEBUG

COMMON /DATA/ DEIZ, PI, GG, KK, CO

COMMON /PROP/ CONE, CPE, IEG, NUE, NUG, RHOE, RHOG, VISE, VISG

COMMON /FLAG/ EBO, EPR, FLM, EKE, EKV, EKS, EFD

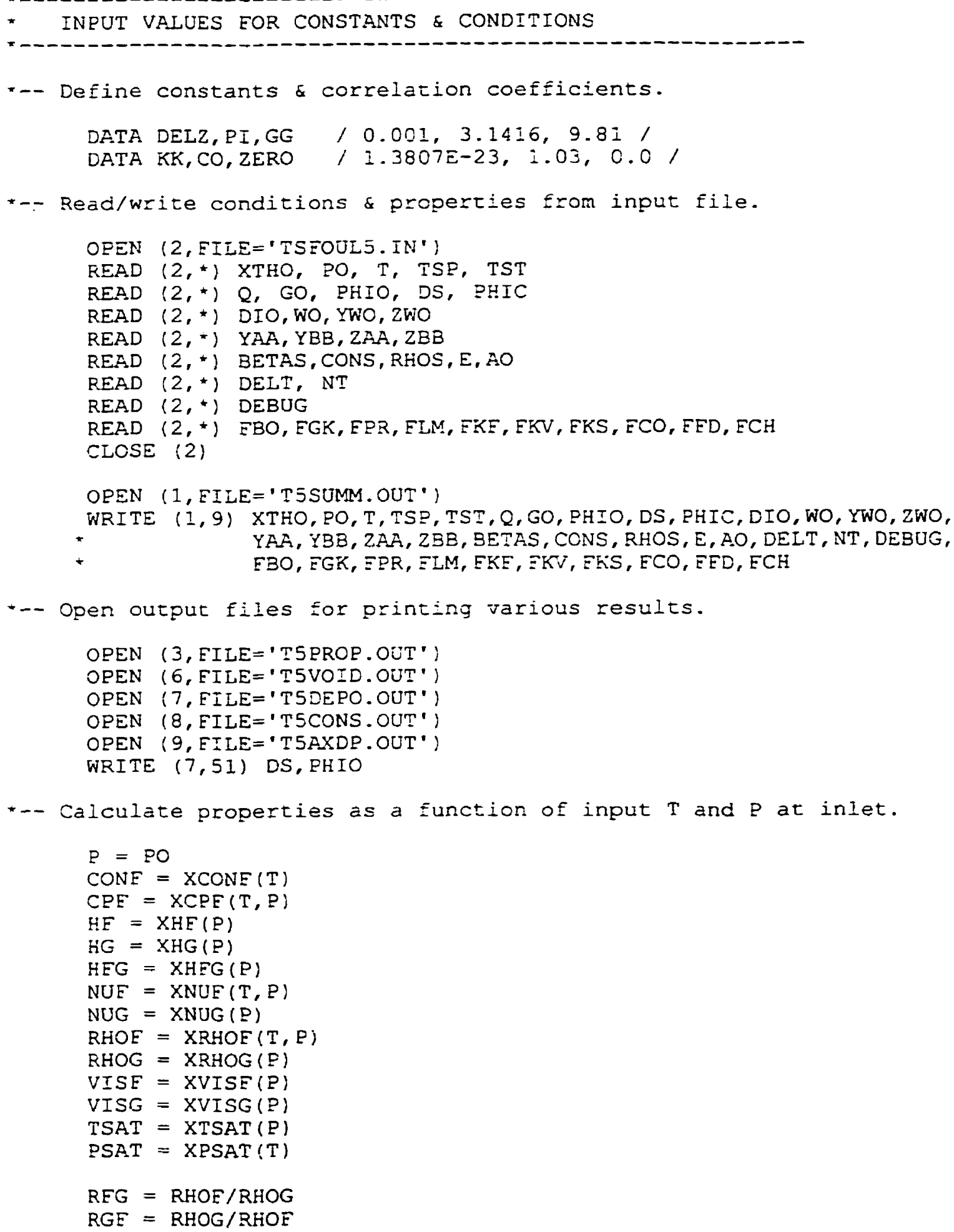

* INPUT VALUES EOR CONSTANTS \& CONDITIONS

-- Define constants \& correlation coefficients.

DATA DELZ,PI,GG / $0.001,3.1416,9.81 /$

DATA KK, CO, ZERO / I.3807E-23, $1.03,0.0 /$

*-- Read/write conditions \& properties from input file.

--- Open output files for printing various results. 


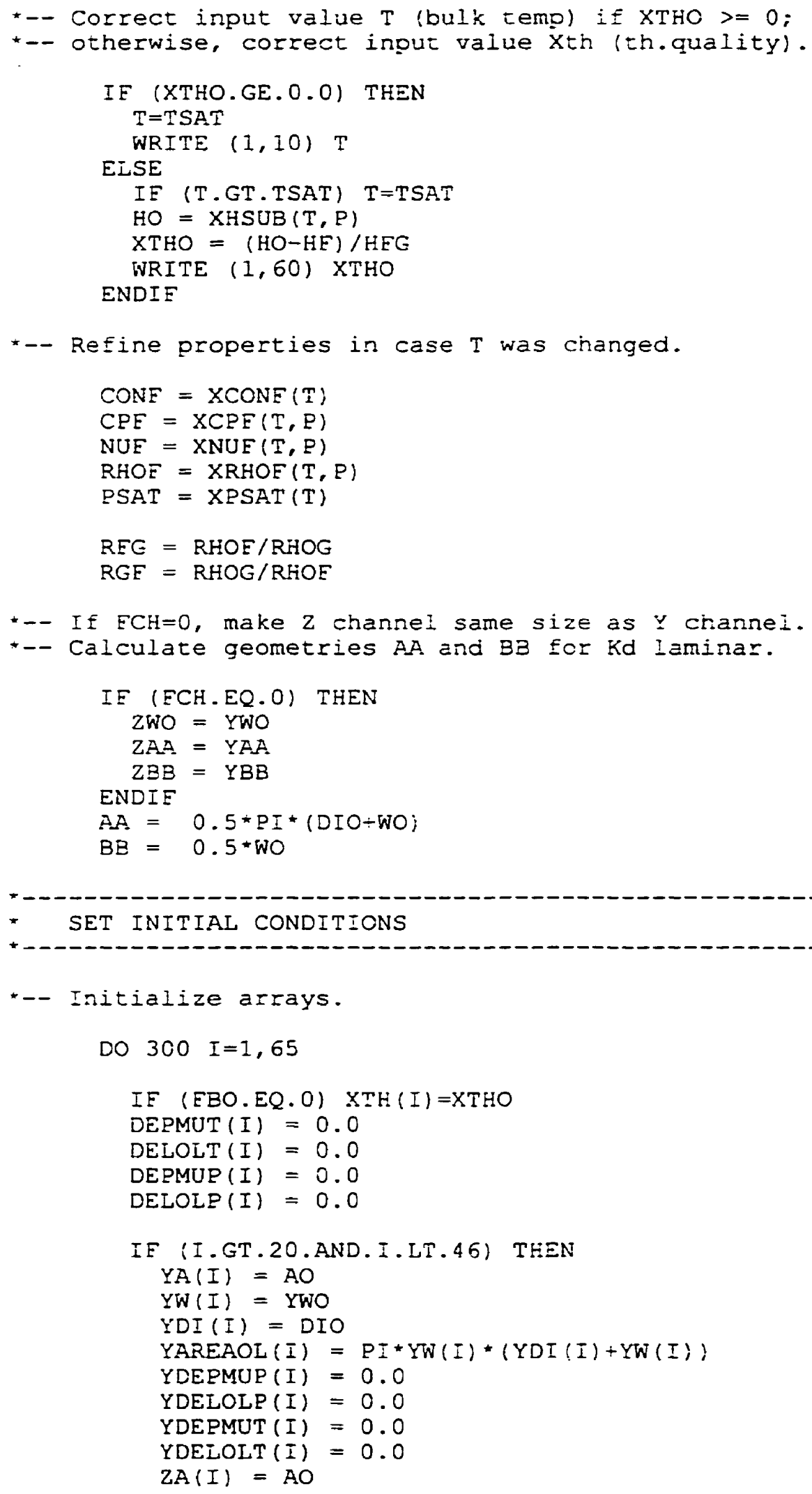




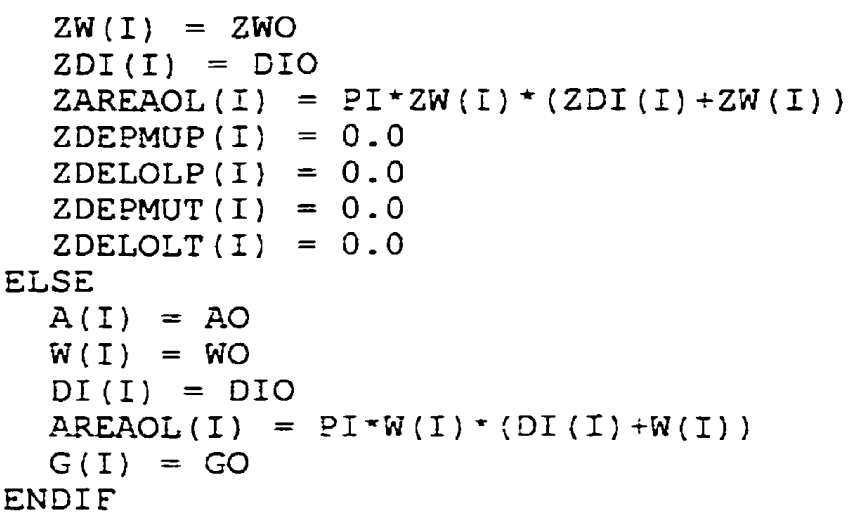

300 CONTINUE

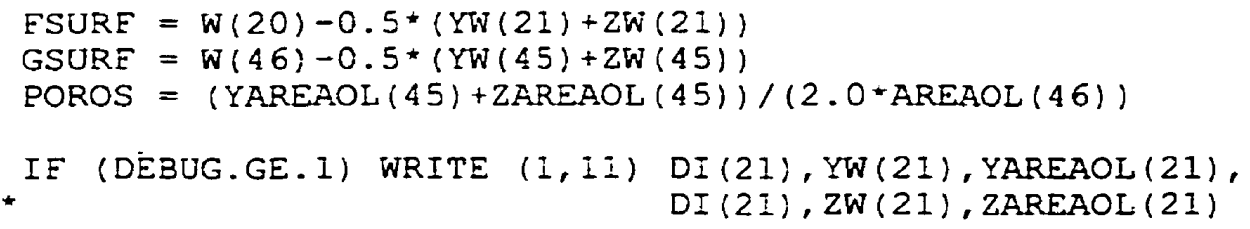

-- Calculate initial conditions at start of free span.

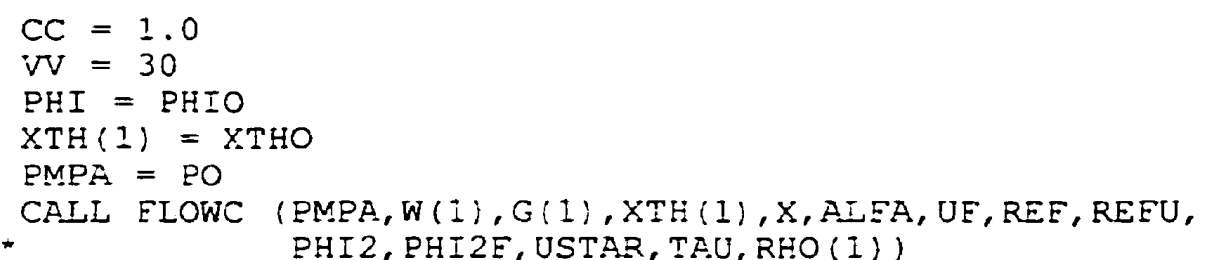

IE (DEBUG.GE.1) WRITE (1, 12) XTH(I),X, ALEA, UE

* start time loop here

*-- Calculate one time step DEIT at a time, Eor NT steps.

DO $200 \mathrm{~J}=1, \mathrm{NT}$

$T T=D E L T * 30.44 * 24.0 * 3600.0 * \operatorname{ELOAT}(\mathrm{J})$

WRITE $(1,13)$ DEIT*FLOAT (J)

WRITE $(7,13)$ DELT*FLOAT $(\mathrm{J})$

WRITE $(1,41)$

WRITE $(9,22)$

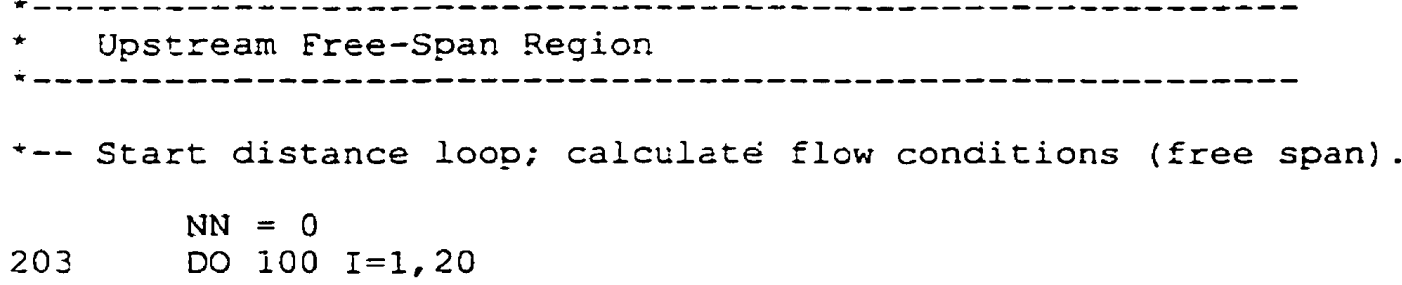




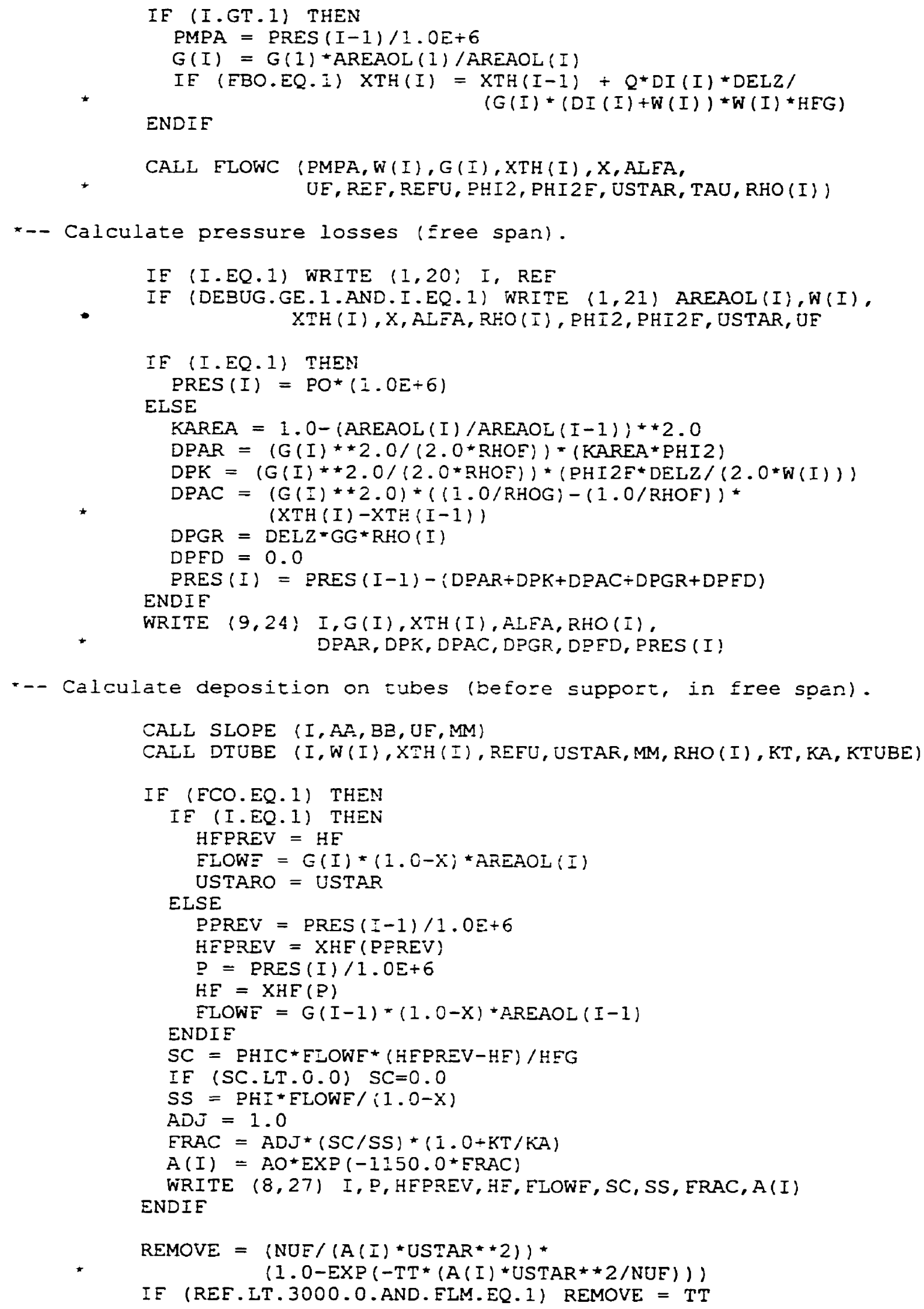




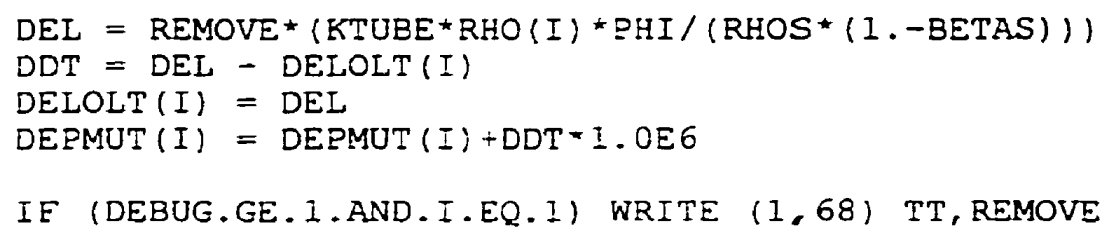

*- Calculate deposition on horizontal bottom of support.

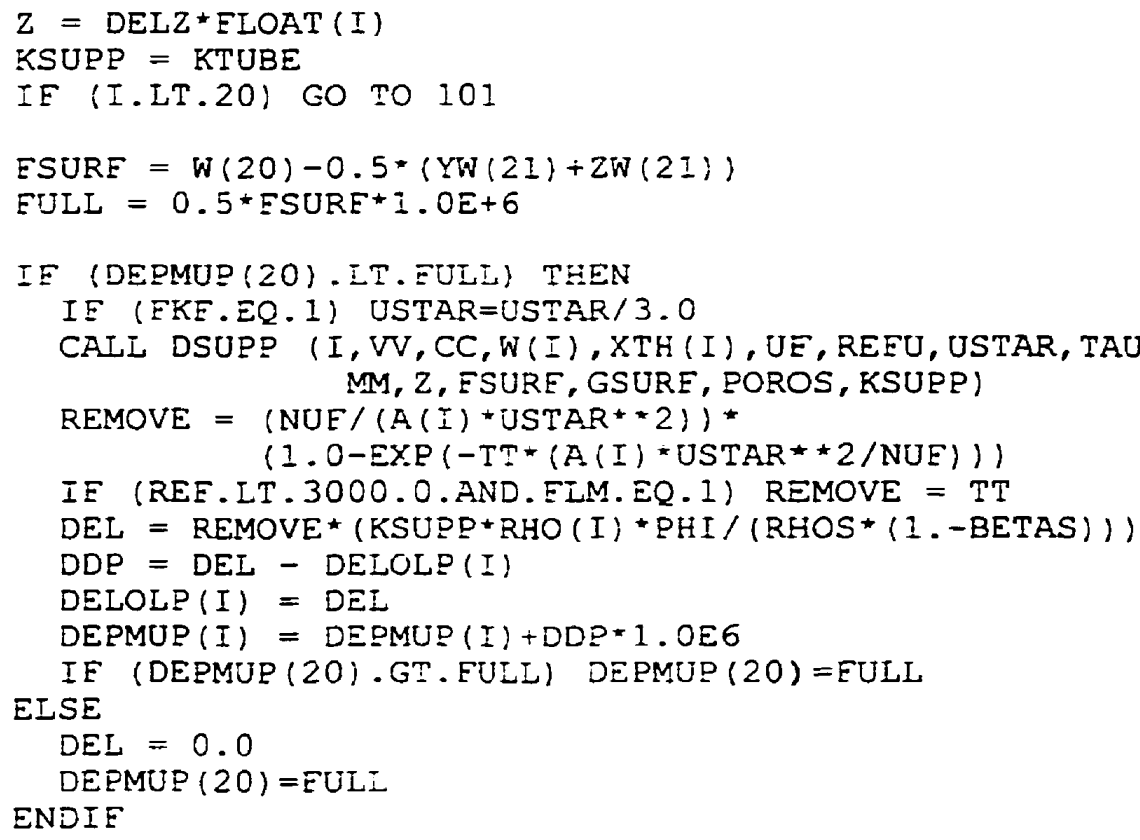




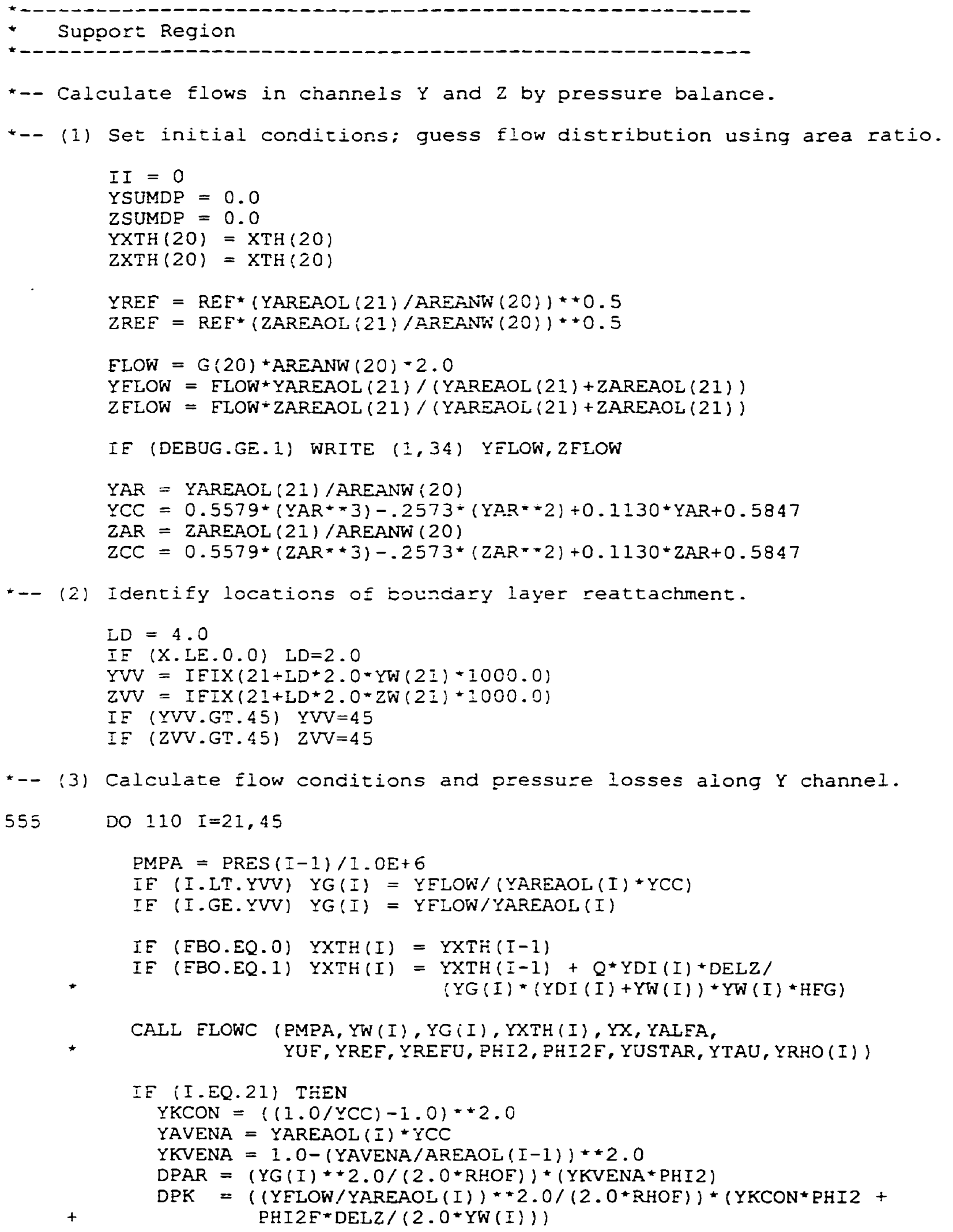




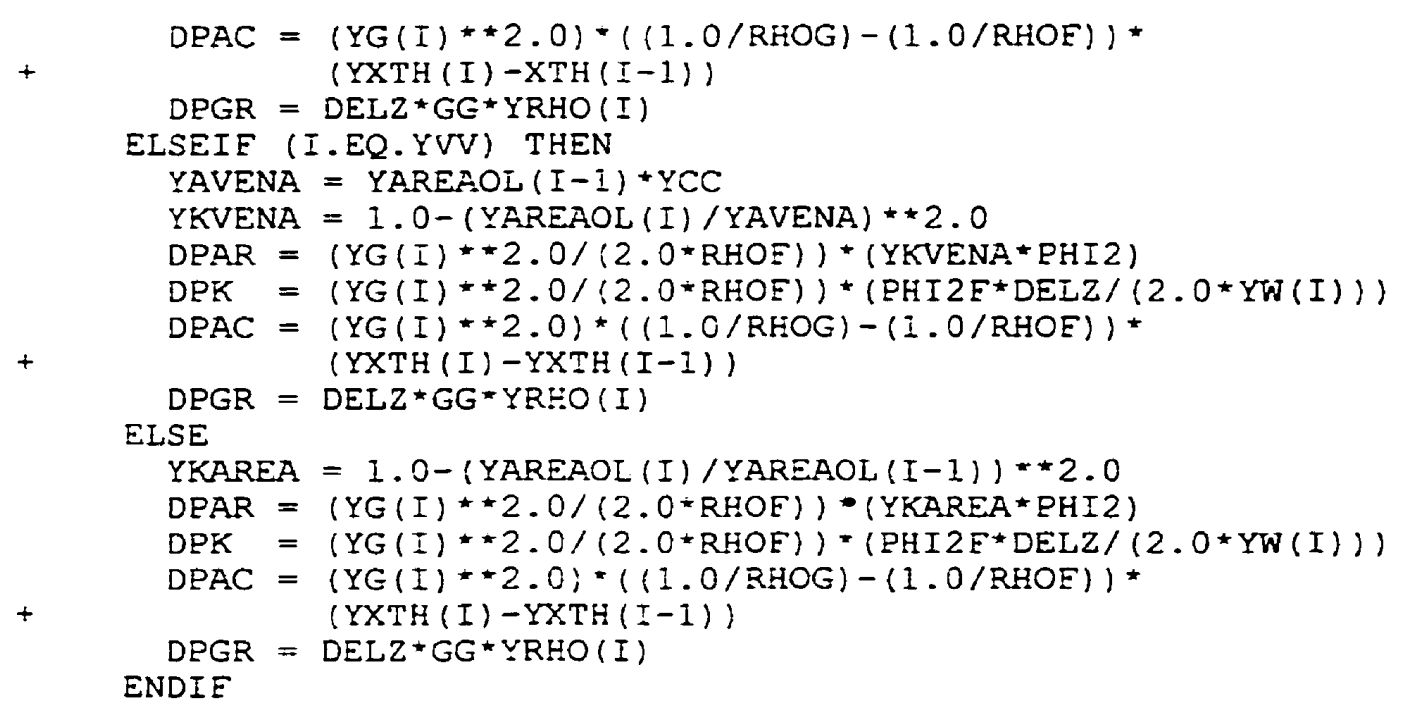

$X X=D E L Z *(E L O A T(I-2 I)) /(2.0 * Y W(I))$
TAVE $=-0.557 * X X * 4+2.6 I * X X * 3-3.49 * X X * 2+0.354 * X+1.33$

DTAVE $=-2.23 * X X * 4+7.84 * X X * 3-6.99 * X X * * 2 \div 0.354$

TLOC = TAVE + DTAVE

IE (TIOC.LT.O.O) ILOC $=0.0$

YKED $=$ TLOC $(1.0-(Y A R E A O I(I) / A R E A O L(20))) *(1.375)$

$D P F D=(Y G(I) * * 2.0 /(2.0 * R H O E)) *(Y K E D * P H I 2)$

YSUMDP = YSUMDP + (DPAR+DPK+DPAC+DPGR+DPED $)$

$\operatorname{PRES}(I)=\operatorname{PRES}(I-1)-(D P A R+D P K+D P A C+D P G R+D P E D)$

WRITE $(9,24) \quad I, Y G(I), Y X T H(I), Y A L E A, Y R H O(I)$, DPAR, DPK, DPAC, DPGR, DPED, PRES (I)

IF (DEBUG.EQ.2) WRITE (1,37) I, DPAR, DPK, DPAC, DPGR, DPED, YSUMDP

(4) Calcuiate flow conditions and pressure losses along $z$ channel.

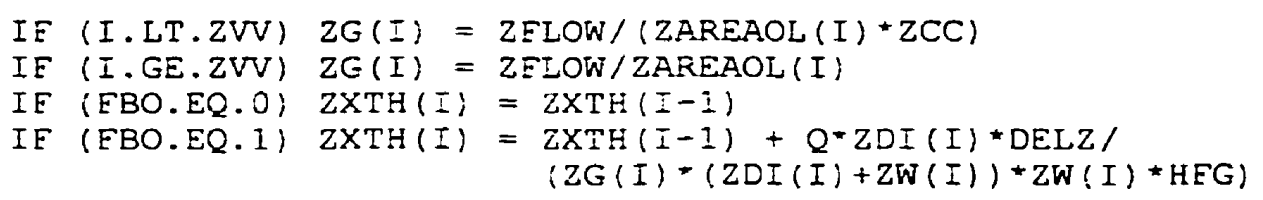

CALL ELOWC (PMPA, ZW(I),ZG(I), ZXTH(I),ZX,ZALFA, ZUE, ZREE, ZREEU, PHI2, PHI2E, ZUSTAR, ZTAU, ZRHO(I))

IF (I.EQ.21) THEN

$2 \mathrm{KCON}=\{(1.0 / Z C C)-1.0) * 2.0$

ZAVENA $=$ ZAREAOL $(I)-Z C C$

ZKVENA $=1.0-(Z A V E N A / A R E A O L(I-1)) * 2.0$

$D P A R=(Z G(I) * 2.0 /(2.0 * R H O E)) *(Z K V E N A * P H I 2)$

$\mathrm{DPK}=((\mathrm{ZFLOW} / \mathrm{ZAREAOL}(I)) * \star 2.0 /(2.0 \star \mathrm{RHOF})) \star(Z K C O N \star \mathrm{PHI} 2+$ $\mathrm{PHI} 2 \mathrm{~F} \times \mathrm{DELZ} /(2.0 \times \mathrm{ZW}(\mathrm{I})))$

$D P A C=(Z G(I) * 2.0) *((1.0 /$ RHOG $)-(1.0 /$ RHOF $)) *$ $(Z X T H(I)-X T H(I-1))$

$D P G R=D E L Z * G G * Z R H O(I)$

ELSEIF (I.EQ.ZVV) THEN

ZAVENA $=$ ZAREAOL $(I-I) * Z C C$

ZKVENA $=1.0-(Z A R E A O L(I) / Z A V E N A) * \star 2.0$

$D P A R=(Z G(I) * 2.0 /(2.0 *$ RHOE $)) *(Z K V E N A * P H I 2)$ 


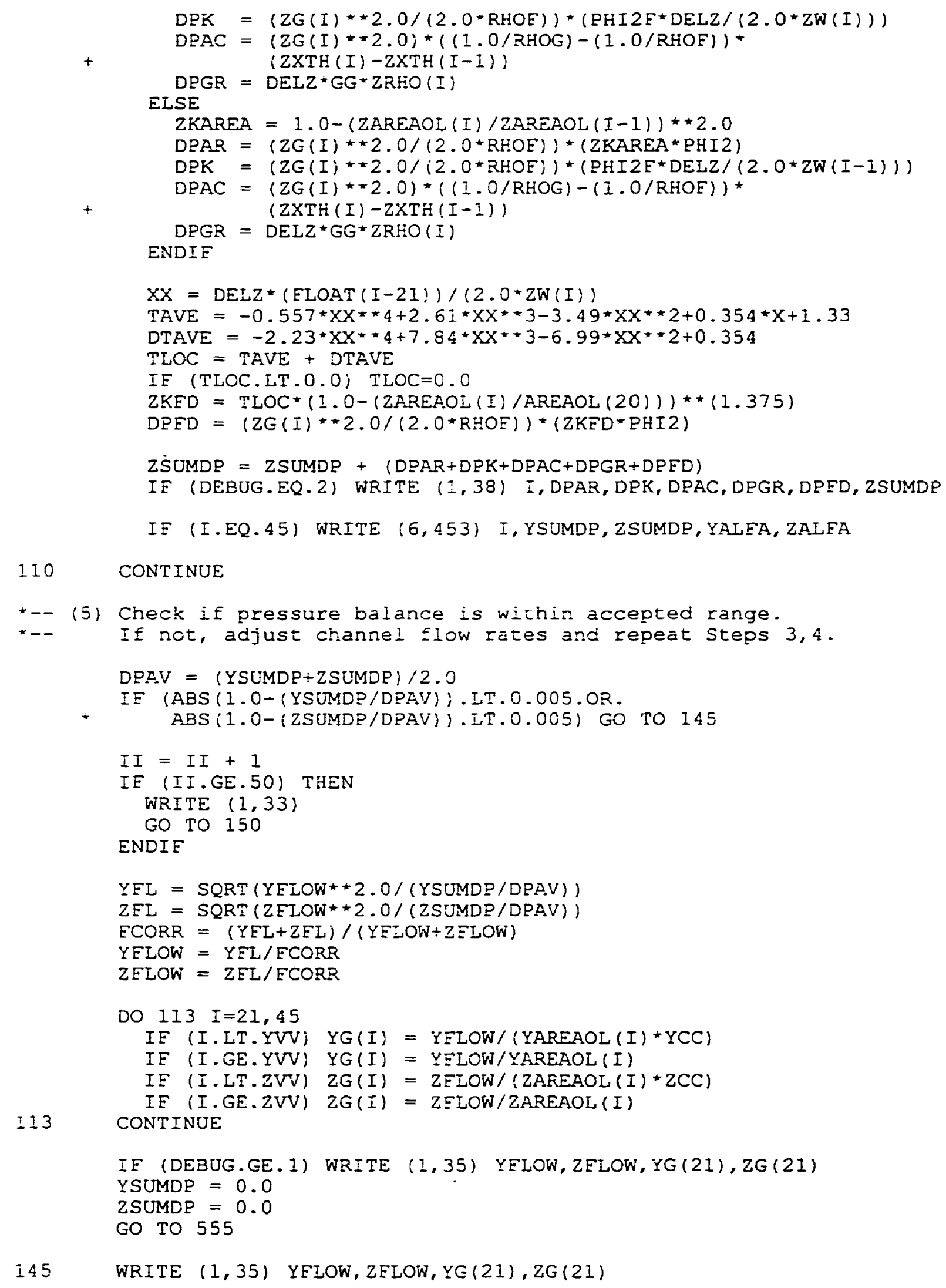


*-- Channel flow rates are now established.

*-- Calculate flow conditions in Y channel.

$$
\text { YPHI }=\text { PHI }
$$

WRITE $(1,44)$

DO $\quad 120 \quad I=21,45$

$$
P M P A=\operatorname{PRES}(I-I) / 1.0 E+6
$$

CALL FLOWC (PMPA, YW(I), YG(I), YXTH(I), YX, YALEA, YUE, YREF, YREEU, PHI2, PHI $2 E, Y U S T A R, Y T A U, Y R H O(I))$

IE (I.EQ.2I) WRITE (I, 20) I, YREF

*- Calculate deposition on heated tube surface in Y channel.

CALL SLOPE (I, YAA, YBE, YUE, YMM)

CALL DTUBE (I, YW(I), YXTH (I), YREEU, YUSTAR, YMM, YRHO(I), $\mathrm{KT}, \mathrm{KA}, \mathrm{KTUBE}$ )

IE (FCO.EQ.I) THEN

PPREV $=$ PRES $(I-1) / 1.0 E+6$

HEPREV $=$ XHF (PPREV)

$\mathrm{P}=$ PRES (I) $/ 1.0 E+6$

$\mathrm{HE}=\operatorname{XHE}(\mathrm{P})$

FLOWE $=$ YELOW* $(1.0-Y X)$

$S C=$ PHIC ${ }^{*}$ LOWE (HFPREV-HE) / HEG

IE (SC.LT.O.O) SC $=0.0$

$S S=Y P H I * E L O W E /(1.0-Y X)$

$\mathrm{ADJ}=1.0$

$E R A C=A D J *(S C / S S) *(1.0+K T / K A)$

IE (I.EQ.2I) ERACYV = ERAC

IE (I.LT.YVV) FRAC = ERACYV/ELOAT $(Y V V-21)$

$Y A(I)=A O * \operatorname{EXP}(-1150.0 * E R A C)$

WRITE $(8,27)$ I, P, EEPREV, $\mathrm{EE}$, ELOWF, SC, SS, ERAC, YA(I)

ENDIF

REMOVE $=(N U E /(Y A(I) * Y U S T A R * \star 2)) *$ (1. O-EXP $(-T T \star(Y A(I) \star Y U S T A R \star \star 2 / N U E))\}$

IE (YREE.IT.3000.0.AND. ELM.EQ.1) REMOVE = TT

$D E I=\operatorname{REMOVE} *(K T U B E * Y R H O(I) * Y P H I /(R H O S *(1 .-B E T A S)))$

$\mathrm{DDT}=\mathrm{DEL}-\mathrm{YDELOLT}(I)$

IE ((DEL+YDELOLP(I)).GE. YW (I)) THEN

$D E I=Y W(I)-Y D E L O L P(I)$

$D D T=Y W(I)-(Y D E L O L T(I)+Y D E L O L P(I))$

ENDIE

YDELOLT $(I)=D E L$

YDEPMUT $(I)=\operatorname{YDEPMUT}(I)+D D T \star 1.0 E 6$

IE (DEBUG.GE.I.AND.I.EQ.21; WRITE $(1,68)$ TT, REMOVE

*-- Calculate deposition on unheated support sirface in $Y$ channel.

$Y Z=D E I Z * E L O A T(I)$

IF (I.LT.YVV.AND. EKV.EQ.I) YUSTAR=YUSTAR/10.0

CALL DSUPP (I, YVV, YCC, YW (I), YXTH (I), YUE, YREEU, YUSTAR, YTAU, YMM, YZ, ESURE, GSURE, POROS, KSUPP) 


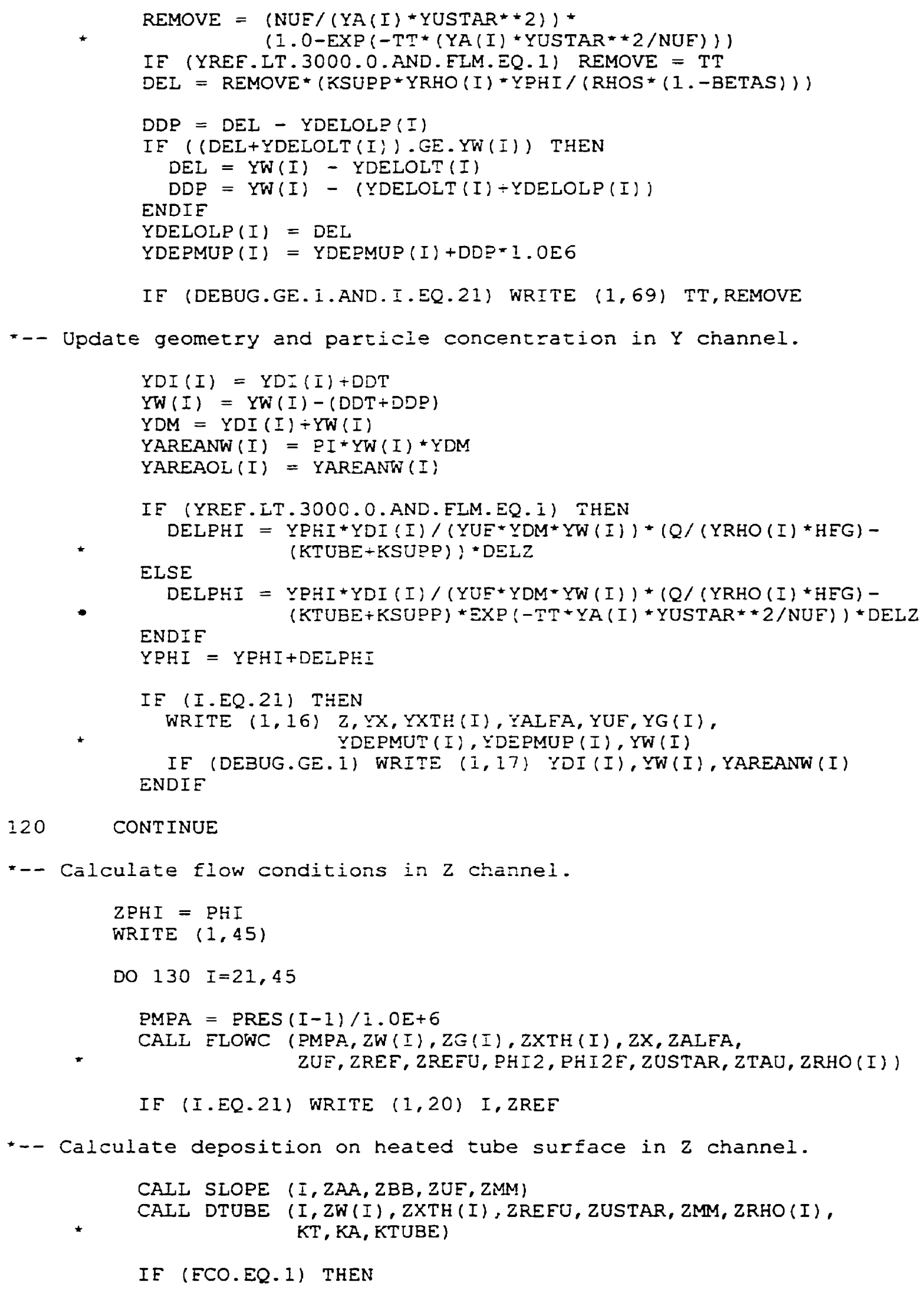




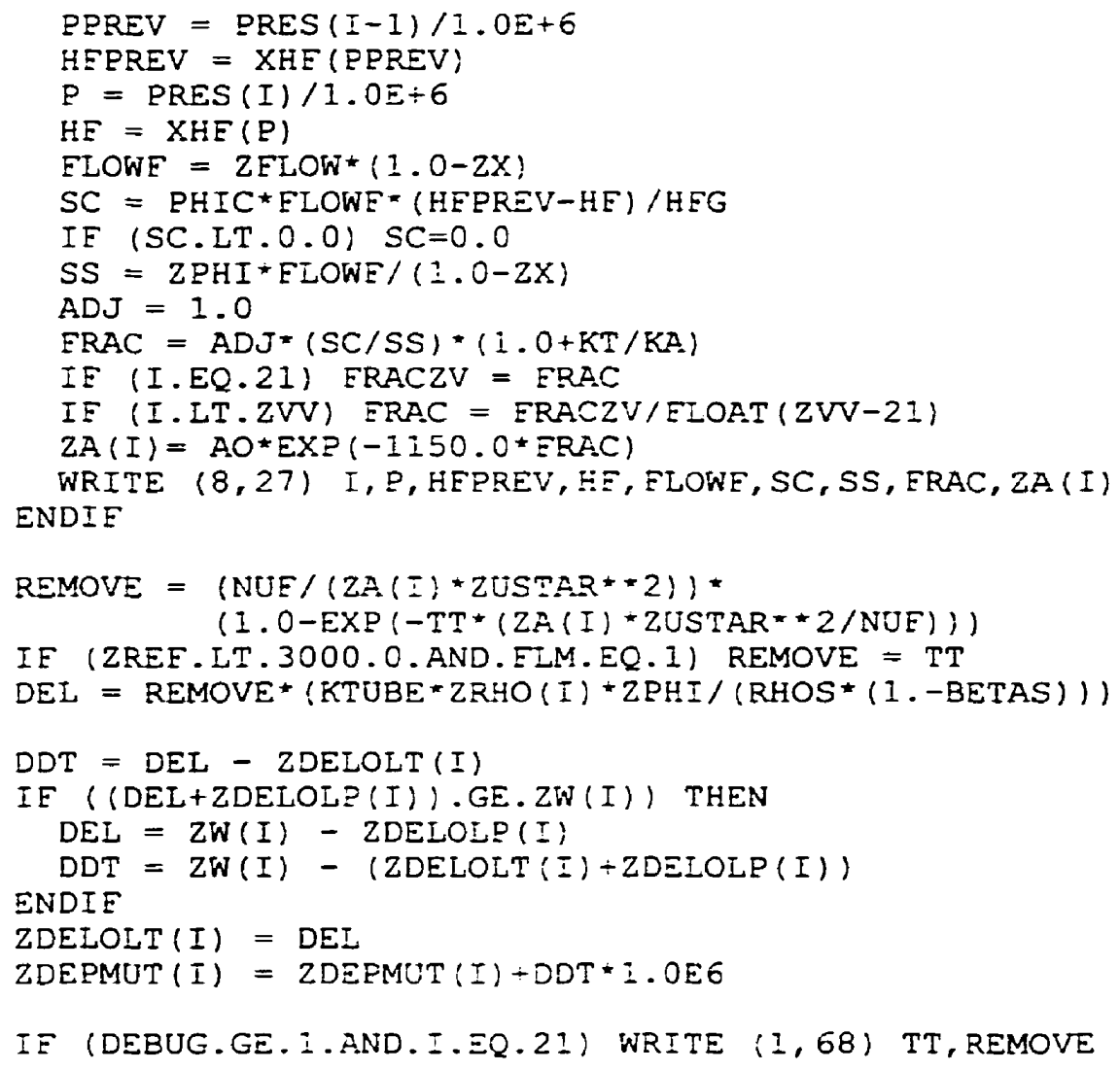




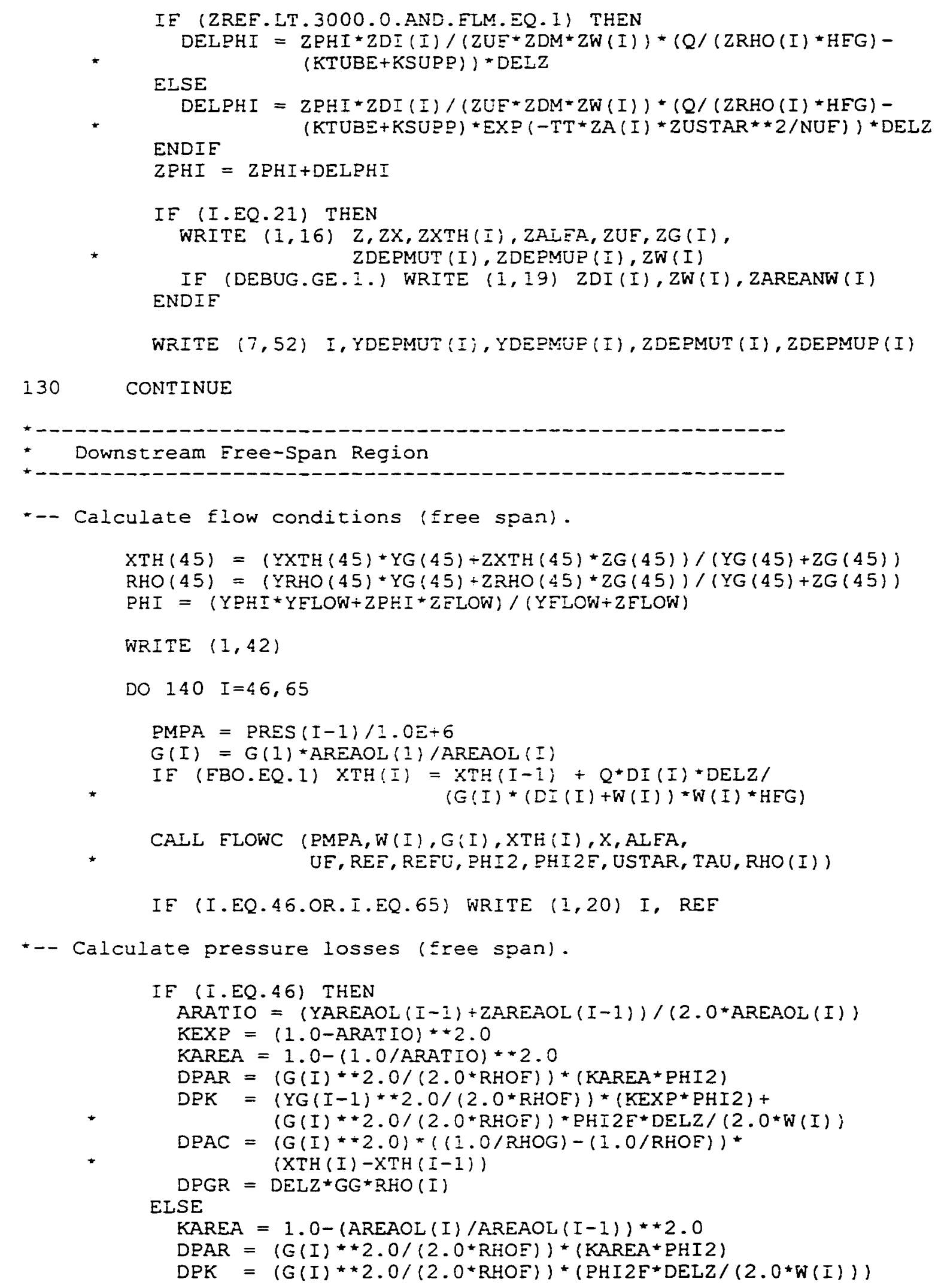




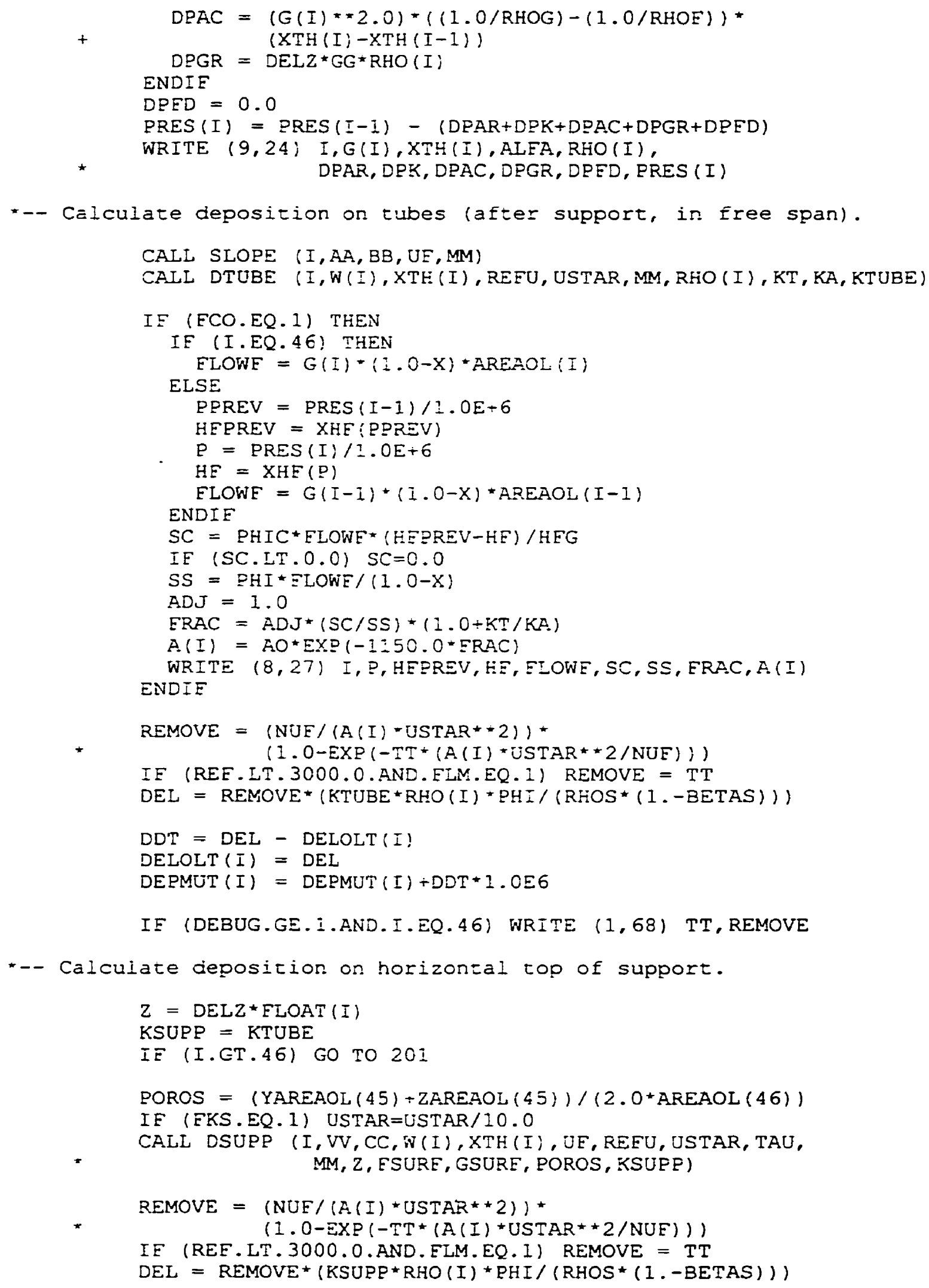




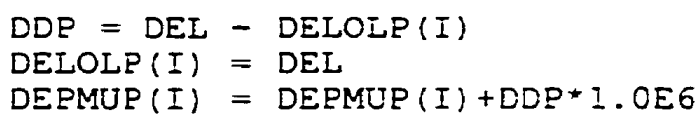

CONTINUE

IE (DEBUG.GE.I.AND.I.EQ.46) WRITE (I.69) TT, REMOVE

-- Update geometry and particle concentration (free span).

$D I(I)=D I(I)+D D T$

$W(I)=W(I)-D D T$

$D M=D I(I)+W(I)$

$A R E A N W(I)=P I \star W(I) \star D M$

AREAOL $(I)=$ AREANW (I)

IE (REE.LT. 3000.0.AND. ELM.EQ.I) THEN

$D E I P H I=P H I * D I(I) /(U \equiv \star D M * W(I)) *(Q /(R H O(I) * H E G)-$

ELSE $2.0 *(K T U B E+K S U P P)) * D E L Z$

$D E L P H I=P H I * D I(I) /(U E * D M * W(I)) \star(Q /(R H O(I) \star H E G)-$

ENDIE

PHI = PHI+DELPHI

IE (I.EQ.46.OR.I.EQ.65) WRITE (1,14) Z,X,XTH(I), ALFA, UE,

WRITE $(7,52)$ I, DEPMUT (I), DEPMUP(I), ZERO, ZERO

I40 CONTINUE

-- Update $G$ based on the decreasing average flow area in the support.

$A D J 2=I .0$

IE (FGK.EQ.i) THEN

IF (J.EQ.1) THEN

DPEIX = PRES (1) - PRES (65)

ELSE

$\mathrm{NN}=\mathrm{NN}+\mathrm{I}$

DPNEW = ERES (I) - ERES (65)

$D D P=D P E I X-D P N E W$

$D G D D P=2 * D P N E W / G(1 ;$

$\mathrm{DG}=\mathrm{DDP} / \mathrm{DGDDP}$

$G(I)=G(i) \div D G \star A D J 2$

$E R R=A B S(D D P / D P N E W)$

WRITE $(3,823)$ J, DPEIX, DPNEW, ERR, G(1)

823 FORMAT (' $J=', I 2, '$ DPEIX $=$ ',E9.3,' DPNEW=',E9.3, - $\left.E R R=1, E 7.4,{ }^{\prime} G I N=1, E 9.1\right)$

IF (ERR.GT.0.01.AND.NN.IT.50) GOTO 203

IE (NN.GE.50) WRITE $(3,824)$

824 FORMAT (' MORE THAN 50 ITERATIONS')

ENDIE

-- Continue calculating for next time step J.

200 CONTINUE

EORMAT STATEMENTS 


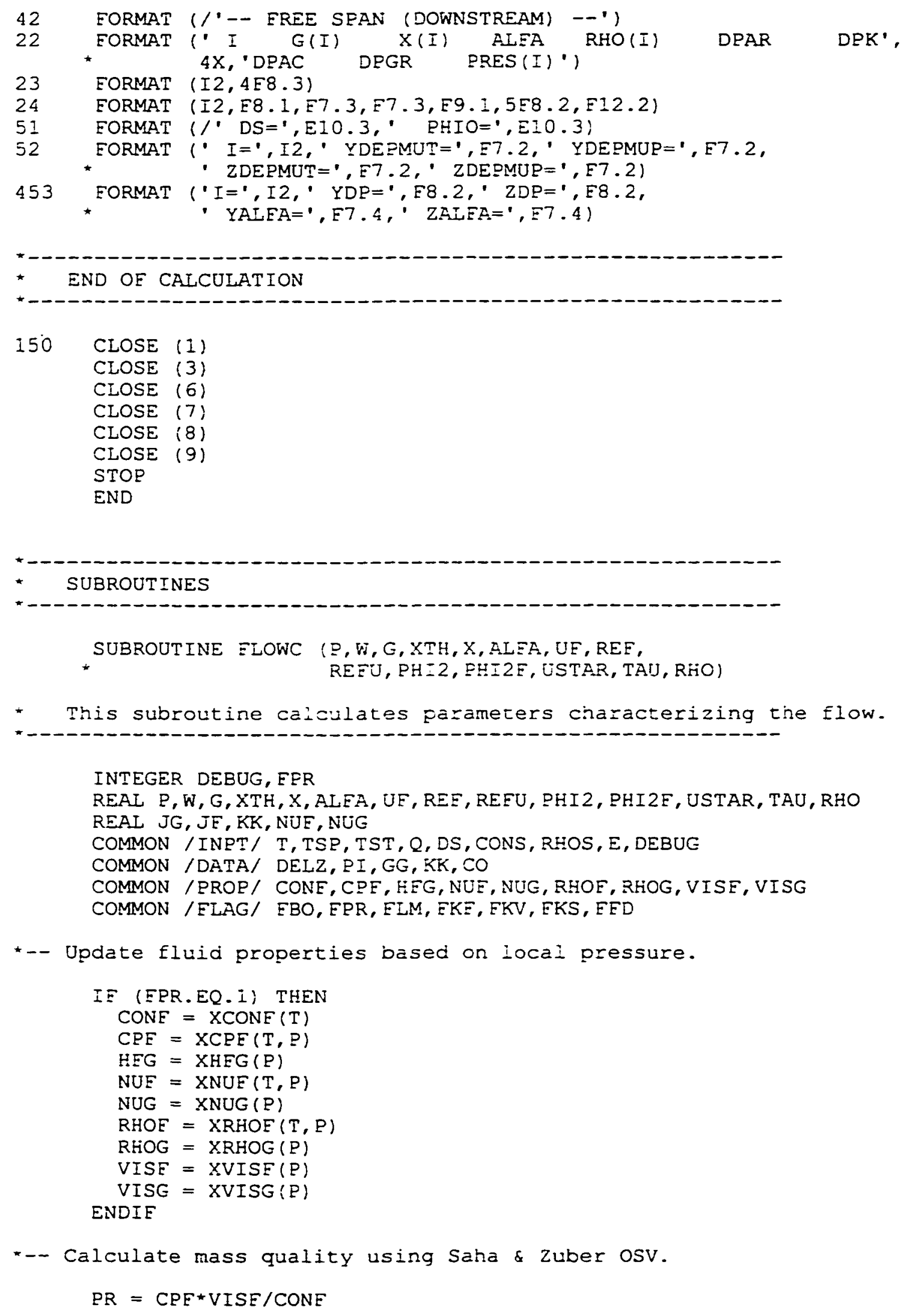




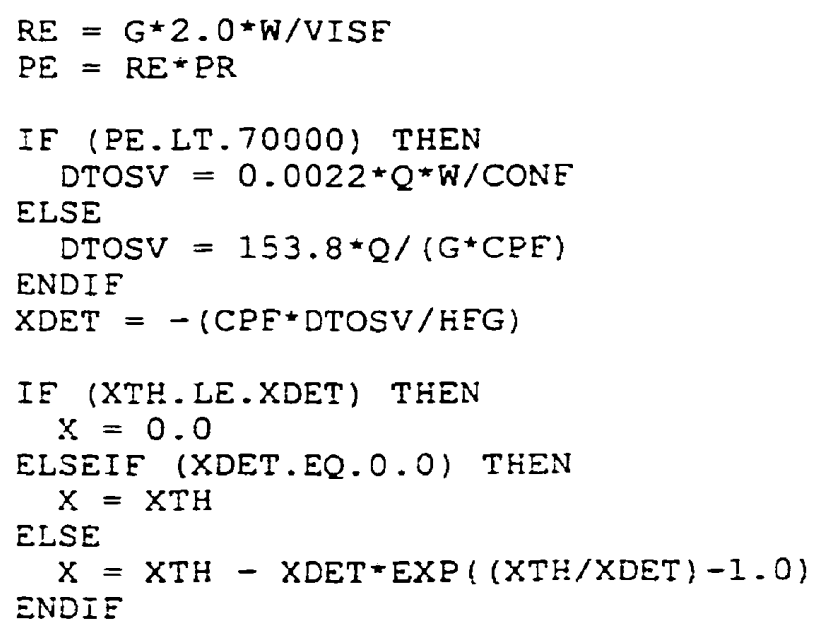

*- Calculate void fraction and velocities.

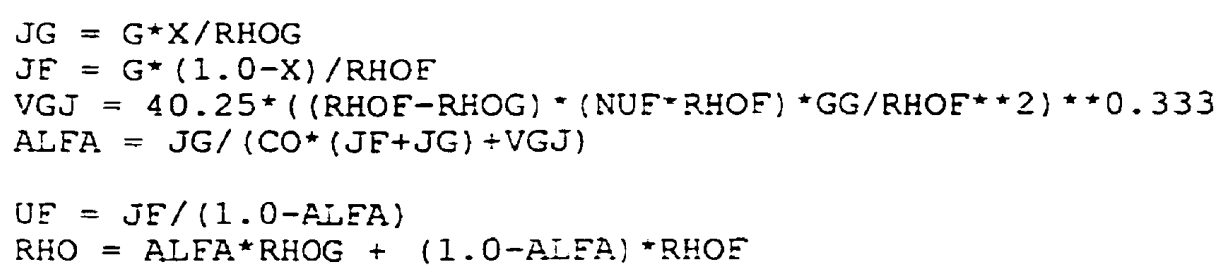

*- Calculate friction factor and two-pnase multiplier (based on Ref).

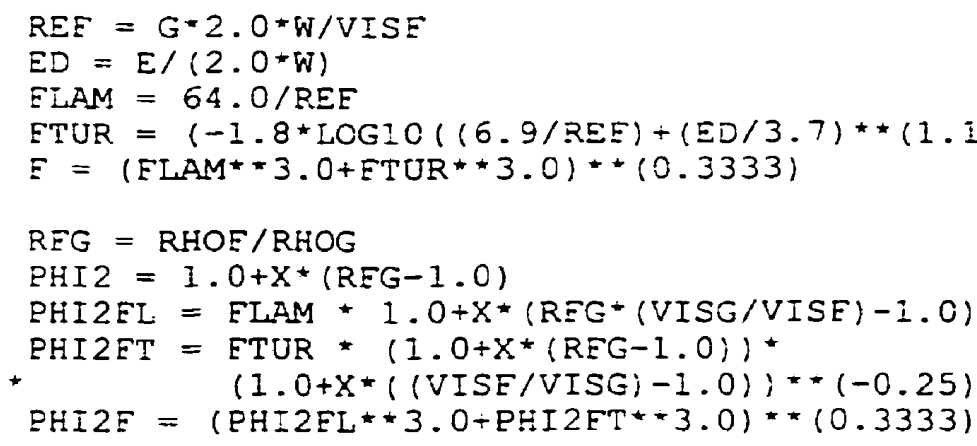

*-- Calculate friction velocity anc shear stress based on Ref(u).

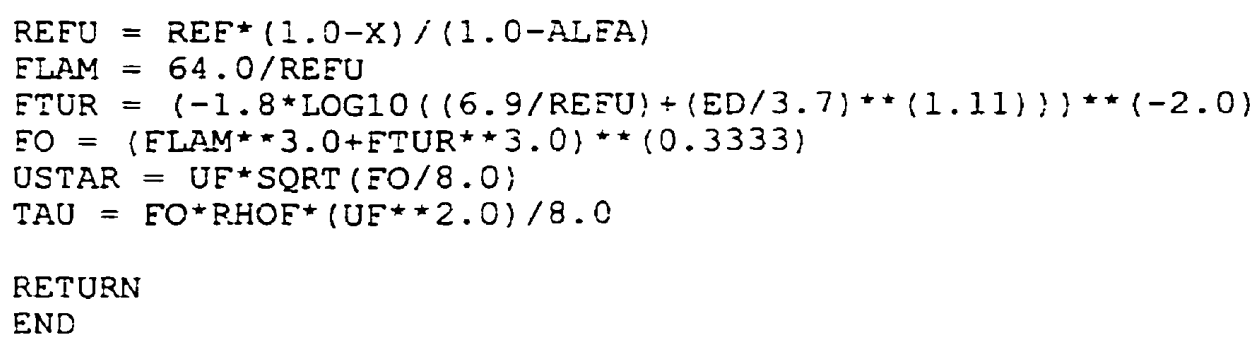


* This subroutine calculates a parameter in the expression

* Eor KD in laminar flow (Vasak)

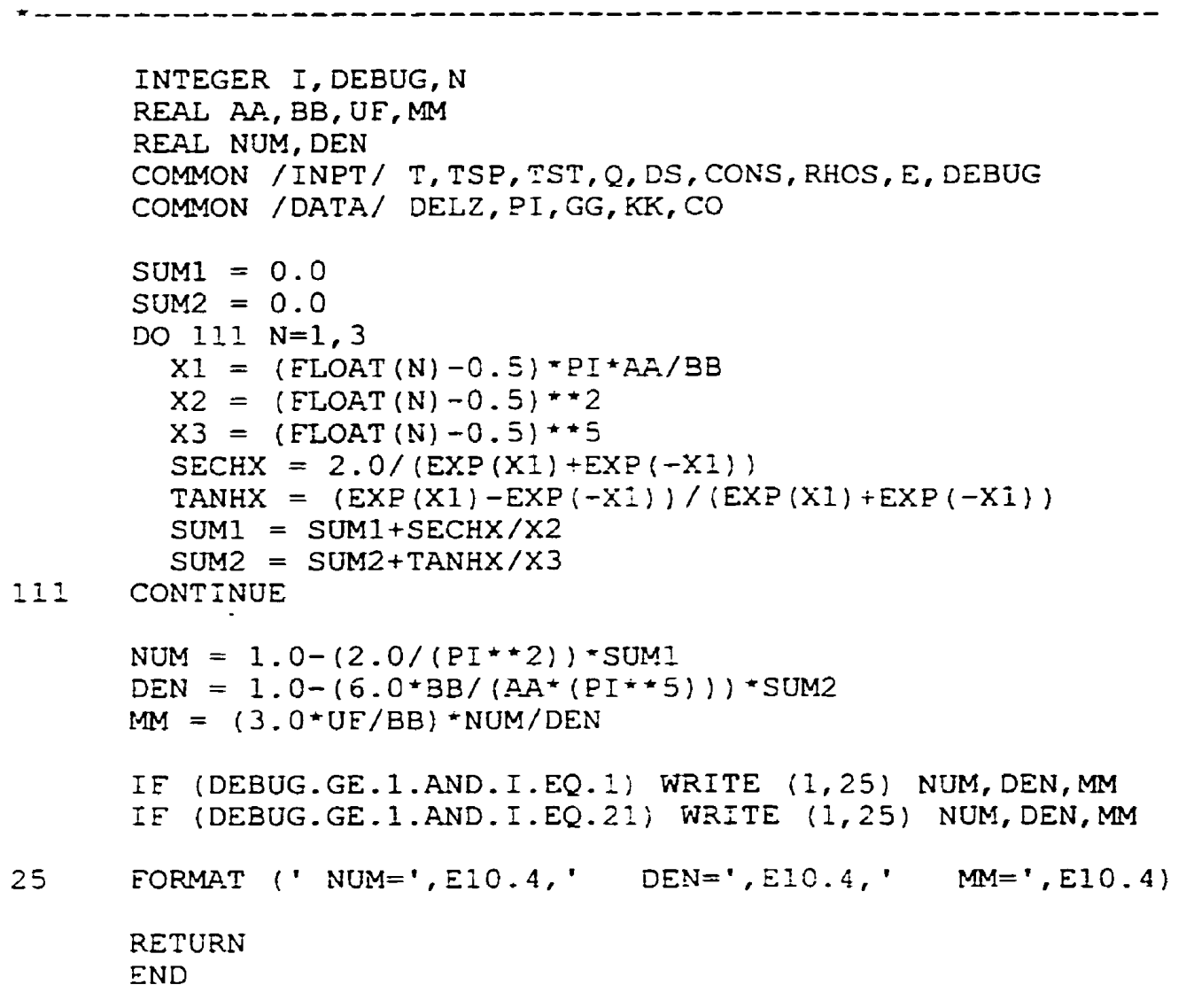

SUBROUTINE DTUEE (I,W, XTH, REFU, USTAR, MM, RHO, KT, KA, KTUBE)

* This subroutine calculates the overall depcsition

- coefficient for the tube surface.

- - -

INTEGER I, DEBUG, FBC, EPR, FLM, EKE, EKV, EKS, EFD

REAL $W, X T H$, REEU, USTAR, MM, RHO, KT, KA, KTUBE.

REAL KTH, KI, KD, KDI, KB, K1P, K2P

REAL KK, NUE, NUG

COMMON /INPT/ T, TSP, TST, Q, DS, CONS, RHOS, E, DEBUG

COMMON /DATA! DELZ, PI, GG,KK,CO

COMMON /PROP/ CONE, CPE, HEG, NUE, NUG, RHOE, RHOG, VISE, VISG

COMMON /ELAG/ EBO, ERR, FLM, EKE, EKV, EKS, EED

*- Track entrance effects for $\mathrm{KD}$ and $\mathrm{KI}$.

$\mathrm{ZDEV}=1.0$

- KD: deposition by diffusion

*- laminar (Vasak et al.) \& turbulent (updated Cleaver \& Yates)

$D=K K \star(T+273.15) /(3.0 \star P I * R H O E \star N U E \star D S)$ 


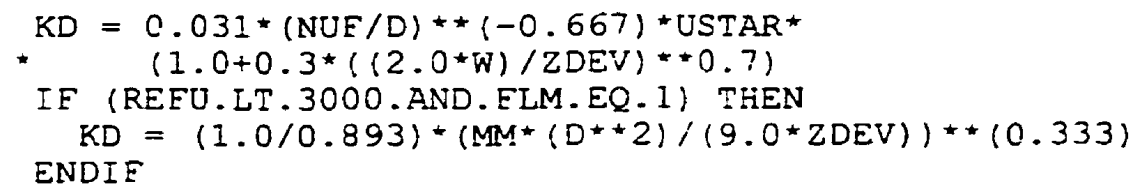

*- KI: deposition by inertial coasting (updated cleaver \& Yates)

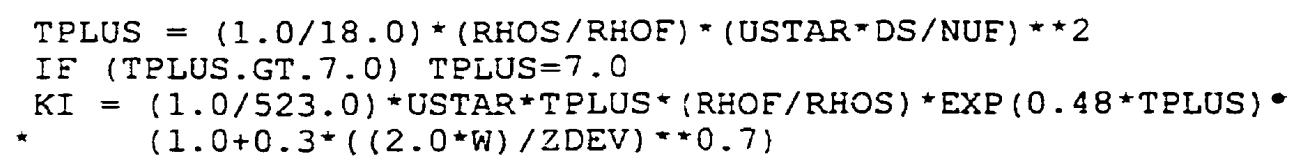

-- KTH: deposition by thermophoresis (McNab \& Meisen)

$\mathrm{KTH}=-0.26 *(1.0 / 6.0) * \mathrm{Q} * \operatorname{NUE} /(\{2.0 * \operatorname{CONE}+\mathrm{CONS}) *(\mathrm{~T} \div 273.15))$

*-- $\mathrm{KB}$ : deposition by boiling (B from Turner \& Godin)

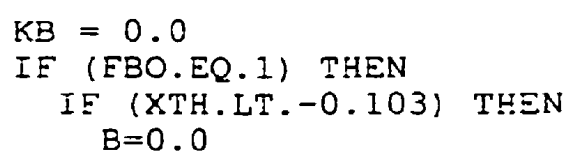

*- KIF, K2P: net deposition coefficient for cubes

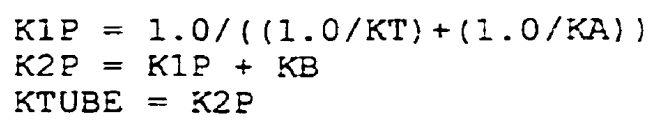


SUBROUTINE DSUPP ( I, VV, CC, $N, X T H, U E, R E F U$, USTAR, TAU,

* MM, Z, ESURE, GSURE, POROS, KSUPP)

* This subroutine calculates the overall deposition * coefficient for the (unisated) support surface.

INTEGER I, VV, DEBUG, EBO, ERR, ELM, EKE, EKV, EKS, EFD

REAI CC, $W, X T H, U F$, REFU, USTAR, TAU, MM, 2

REAI ESURE, GSURE, POROS, KSUPP

REAL KTH, KI, KD, KE, KV, KG, KC, KT, KA, KIP

REAI KK, NUE, NUG, NSTI, NIMP

COMMON /INPT/ T, TSP, TST, Q, DS, CONS, RHOS, E, DEBUG

COMMON /DATA/ DELZ, PI, GG, KK, CO

COMMON / PROP/ CONE, CPE, HEG, NUE, NUG, RHOE, RHOG, VISE, VISG

COMMON /ELAG/ EBO, EPR, ELM, FKE, EKV, EKS, EED

*-- Track entrance effects for $\mathrm{KD}$ and $\mathrm{KI}$.

ZATT $=$ ELOAT $(\mathrm{VV}) / 1000.0$

$\mathrm{ZDEV}=1.0$

IE (EED.EQ.1) THEN

ENDIE

IF (I.GE.VV.AND.I.IT.46) ZDEV=Z-(ZATT-0.001)

*- KD: deposition by diffusion

*- laminar (Vasak et al.) a turbulent (updated Cleaver \& Yates)

$D=K K \star(T+273.15) /(3.0 * P I * R H O E * N U E * D S)$

$K D=0.031 *(N U E / D) * *(-0.667) *$ USTAR

$+\quad(1.0+0.3 *((2.0 * W) / Z D E V) * 0.7)$

IE (REFU.LT.3000.AND. FIM.EQ.1) THEN

$K D=(i .0 / 0.893) \star(M M *(D * 2) /(9.0 * Z D E V)) \star(0.333)$

ENDIE

IE (EED.EQ.1) THEN

ENDIE

IE (I.GE.2I.AND.I.II.VV) KD=0.0

*-- KI: deposition by inertial coasting (updated Cleaver \& Yates)

TPLUS $=(1.0 / 18.0) \bullet($ RHOS $/$ RHOF $) \star(U S T A R \star D S / N U E) \star 2$

IE (TPLUS.GT.7.0) TPLUS $=7.0$

$K I=(1.0 / 523.0) * U S T A R * T P L U S *(R H O E / R H O S) * E X P(0.48 * T P L U S) \star$

- $(1.0+0.3 *((2.0 * W) / Z D E V) * 0.7)$

IE (EED.EQ.I) THEN

ENDIE

IE (I.GE.2I.AND.I.LT.VV) KI=0.0

*-- KTH: deposition by thermophoresis

$\mathrm{KTH}=0.0$

*-- KF: deposition by Eiltering/impaction upstream (Ranz \& Wong)

$K E=0.0$

WJET $=0.002$ 


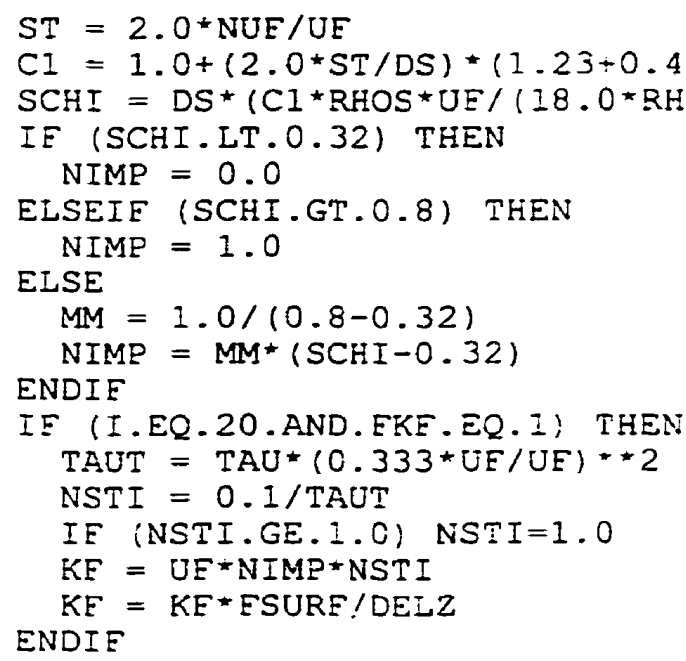

*- KC: deposition by centrifugal settling in vena contracta

--- (Stoke's Law and variation)

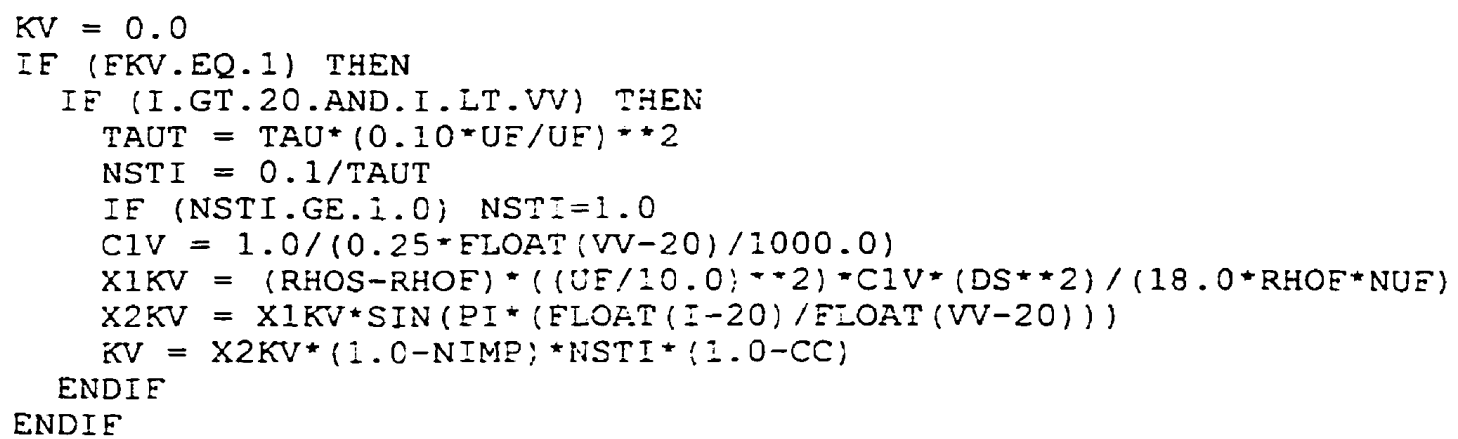

*- KG,KC: deposition by gravitional or centrifugal settling

*- on top of support (Stoke's Law and variation)

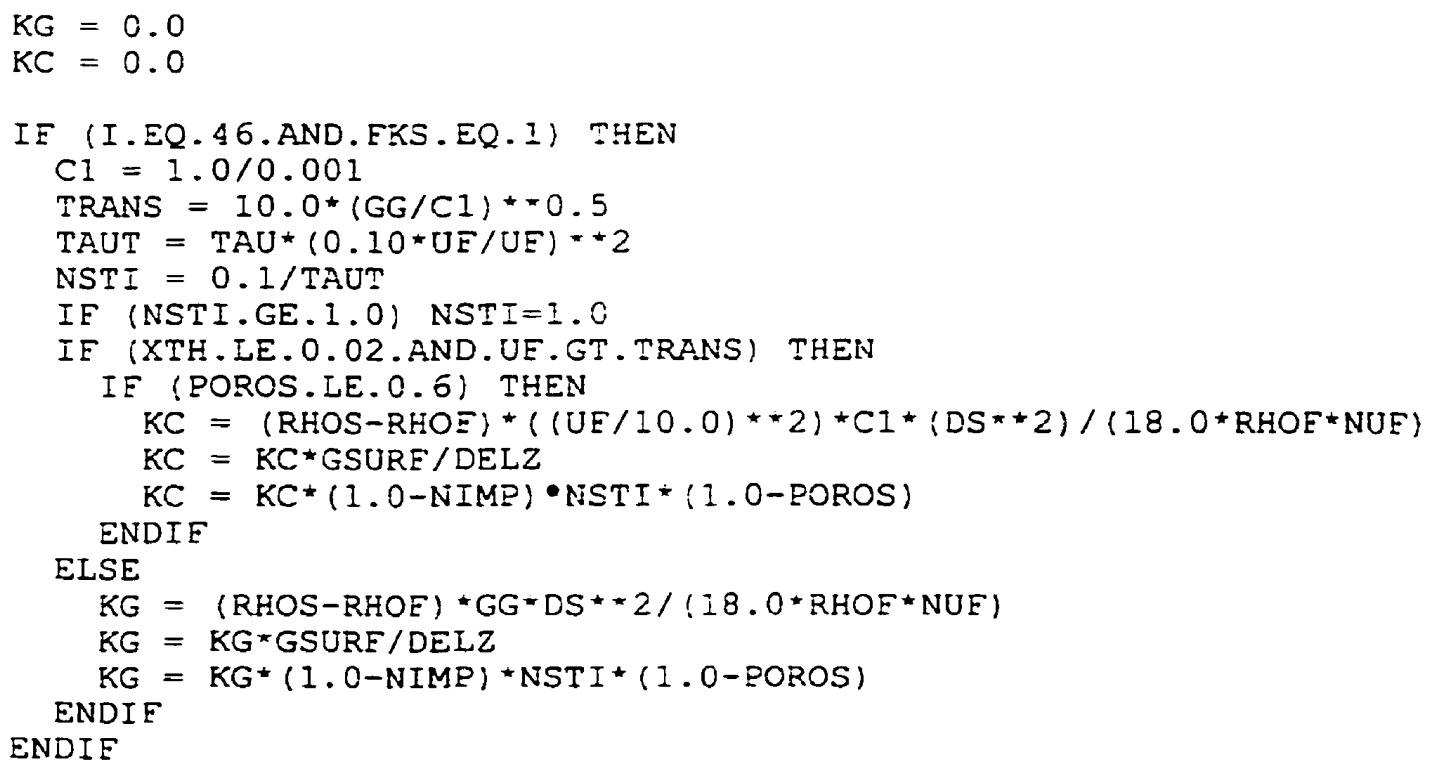




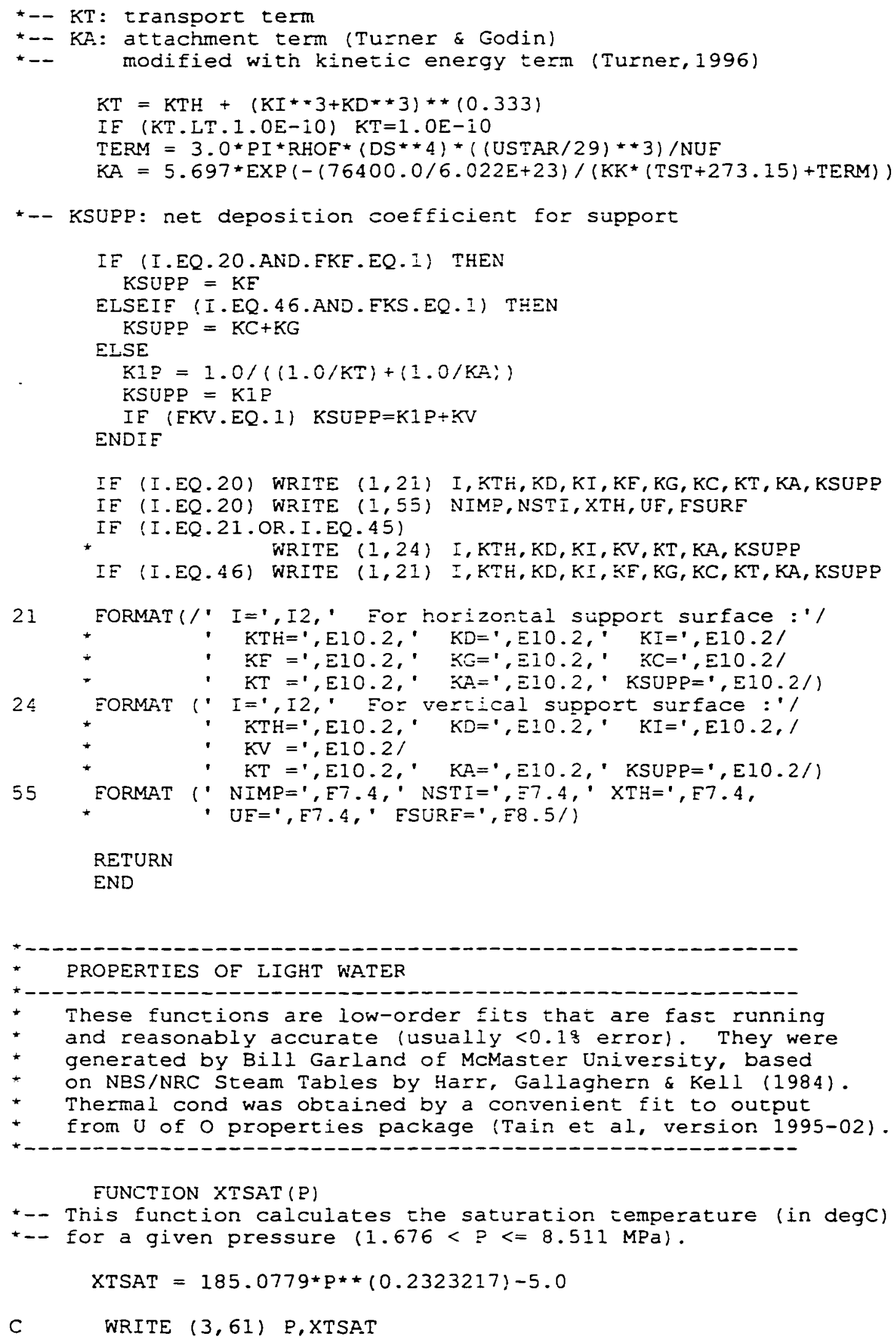




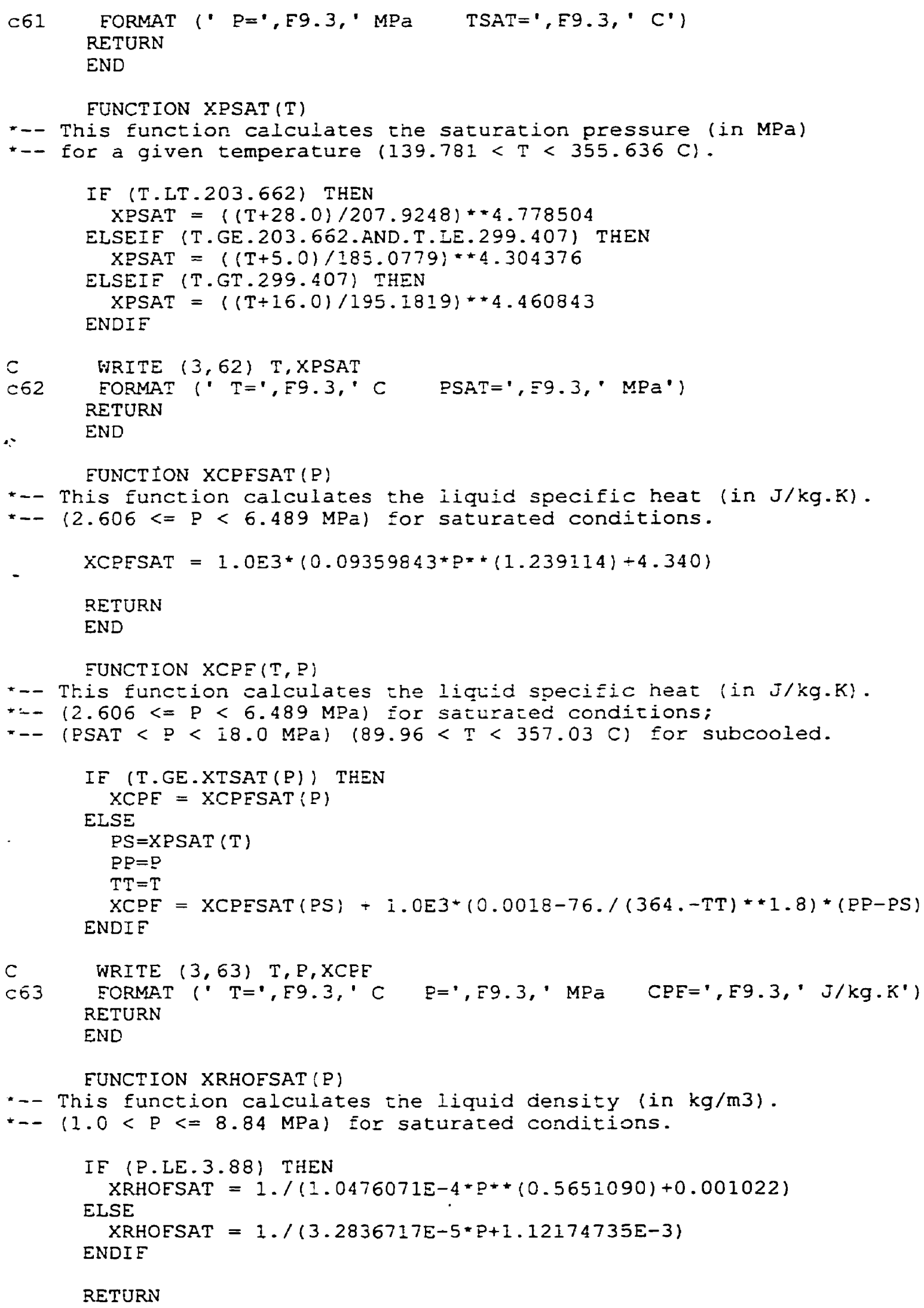


END

FUNCTION XRFOE $(T, P)$

-- This function calculates the liquid density (in $\mathrm{kg} / \mathrm{m} 3$ ).

*- $(1.0<P<=8.84 \mathrm{MPa})$ for saturated conditions;

*- (PSAT < $\mathrm{P}<18.0 \mathrm{MPa})(91.79<\mathrm{T}<357.03 \mathrm{C}$ ) for subcooled.

IE (T.GE.XTSAT (P)) THEN

$X R H O F=X R H O E S A T(P)$

ELSE

PS $=X P S A T(T)$

$\mathrm{PP}=\mathrm{P}$

$T T=T$

XRHOF $=$ XRHOESAT $(P S)+(170.0 /(375.0-T T)-0.2) \cdot(P P-P S)$

ENDIF

C WRITE $(3,64)$ T, P,XRHOE

C64 EORMAT (' $T={ }^{\prime}, E 9.3,^{\prime} \mathrm{C} \quad \mathrm{P}={ }^{\prime}, \mathrm{F9} .3^{\prime},^{\prime} \mathrm{MPa}$ RHOF=', F9.3,' $\mathrm{kg} / \mathrm{m} 3^{\prime}$ ) RETURN

END

EUNCTION XRHOG (P)

*- This function calculates the density (in $\mathrm{kg} / \mathrm{m} 3$ )

*-- in the gas phase at saturation for a given pressure

$*-1.112 \Leftrightarrow P<8.996 \mathrm{MPa})$.

IF (P.LT.3.932) THEN

$X R H O G=4.6308322^{*} *(I .038819)+0.52$

ELSE

$X R H O G=2.868721 * P * *(1.252148)+3.30$

ENDIE

C WRITE $(3,65)$ P, XRHOG

C65 FORMAT (' $P=$ ', $^{\circ}$ 9.3,' MPa RHOG=', E9.3,' $\mathrm{kg} / \mathrm{m} 3$ ')

RETURN

END

FUNCTION XHE (P)

*- This function calculates the liquid specific enthalpy (in $\mathrm{J} / \mathrm{kg}$ ).

*- $(0.942<=P<9.964 \mathrm{MPa})$ for saturated conditions.

IE (P.LT.4.02) THEN

$X H E=638.0621 * P \star *(0.2963192) \div 125.0$

ELSE

$X H E=373.7665 * P * *(0.4235532)+415.0$

ENDIE

$X H E=1.0 E 3 * X H E$

RETURN

END

EUNCTION XHSUE (T, P)

- This function calculates the liquid specific enthalpy (in $\mathrm{J} / \mathrm{kg}$ )

*-- (PSAT < $\mathrm{P}<18.0 \mathrm{MPa)}(91.79<T<357.03 \mathrm{C}$ ) for subcooled.

$P S=X P S A T(T)$

$\mathrm{PP}=\mathrm{P}$

$\mathrm{TT}=\mathrm{T}$

XHSUB $=$ XHE (PS) $+1.0 E 3 *(1.4-169 . /(369 .-T T)) *(P P-P S)$ 


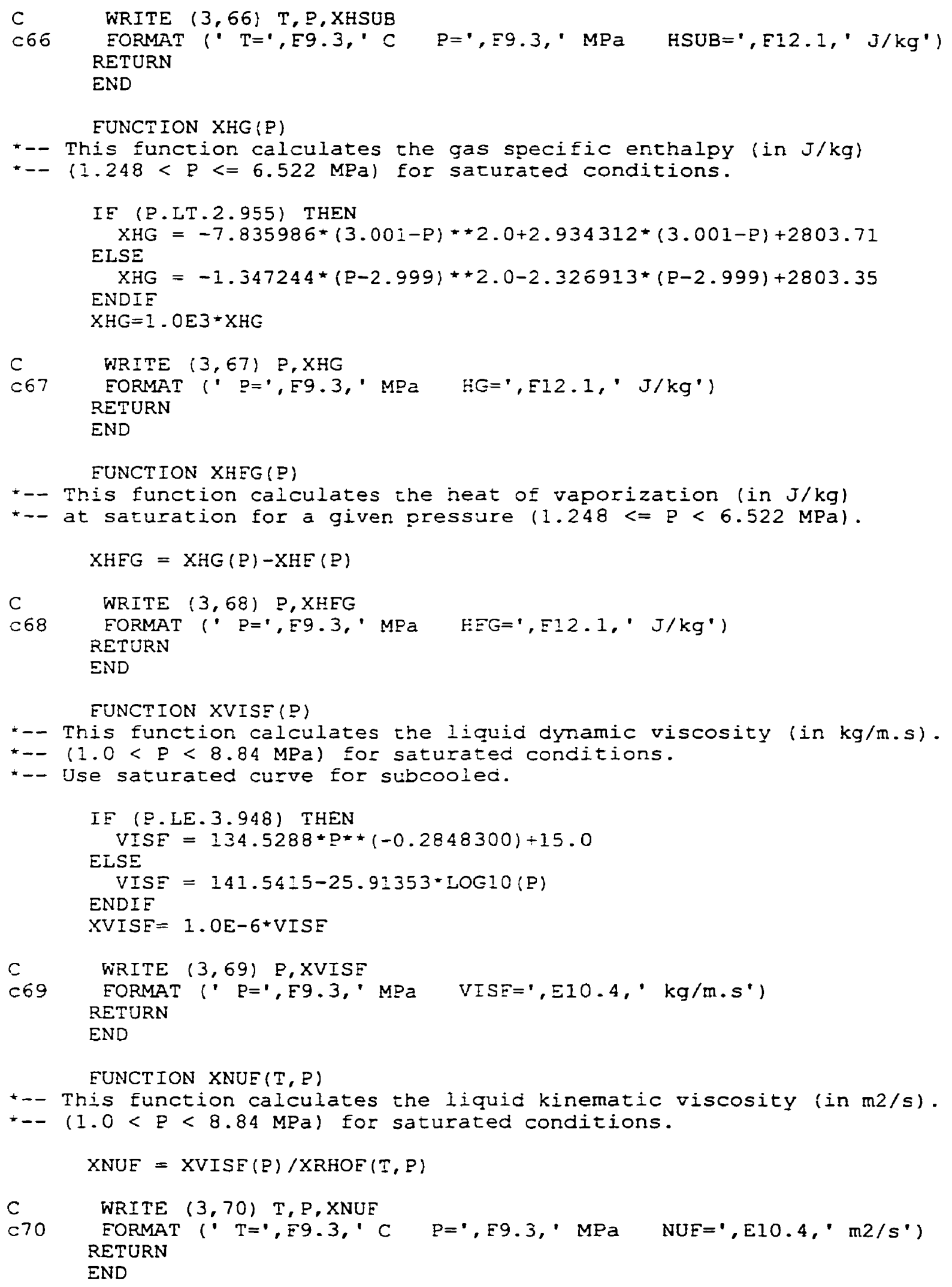


FUNCTION XVISG (P)

*- This function calculates the gas kinematic viscosity (in $\mathrm{m} 2 / \mathrm{s}$ )

*-- $(2.207<P<8.996 \mathrm{MPa})$ for saturated conditions.

IE (P.LE.5.480) THEN

VISG $=3.375163^{\star} P^{\star \star}(0.3916208)+11.8$

ELSE

VISG $=0.9169410 * P * *(0.7644731)+15.0$

ENDIF

$X V I S G=1.0 E-6 * V I S G$

C WRITE $(3,7 I)$ P,XVISG

C71 EORMAT ('P=',E9.3,' MPa VISG=',E9.3.' kg/m.5') RETURN

END

FUNCTION XNUG (P)

*- This function calculates the gas kinematic viscosity (in $\mathrm{m} 2 / \mathrm{s}$ )

*-- $(2.207<\mathrm{P}<8.996 \mathrm{MPa})$ for saturated conditions.

$\mathrm{XNUG}=\mathrm{XVISG}(\mathrm{P}) / \mathrm{XRHOG}(\mathrm{P})$

$C$ WRITE $(3,72)$ P,XNUG

C72 FORMAT (' $\mathrm{P}=\prime^{\prime}, \mathrm{E9} .3$, $\left.^{\prime} \mathrm{MPa} \quad \mathrm{NUG}=\cdot, \mathrm{Eg} .3,^{\prime} \mathrm{m} 2 / \mathrm{s}^{\prime}\right)$ RETURN

END

EUNCTION XCONF(T)

*-- This function calculates the liquid thermal conductivity (in $\mathrm{J} / \mathrm{s} . \mathrm{m} . \mathrm{K}$ ).

- $(4.4<D<4.6 \mathrm{MPa}, 200<T<260 \mathrm{C})$.

$X \operatorname{CONE}=-6.265 E-6 * T * T+0.0018 * T \div 0.5581$

C WRITE $(3,73) T, X$ CONE

C73 EORMAT (' T=",E9.3,' C CONE=',E9.3,' J/kg.m.s') RETURN

END 


\section{APPENDIX D: TYPICAL INPUT FILE FOR TSFOUL}

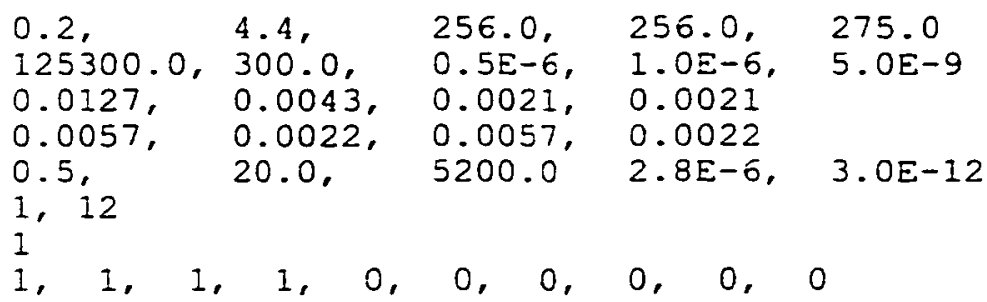

"EBO, EGK, EPR, ELM, EKE, EKV, EKS, ECO, FED, ECH

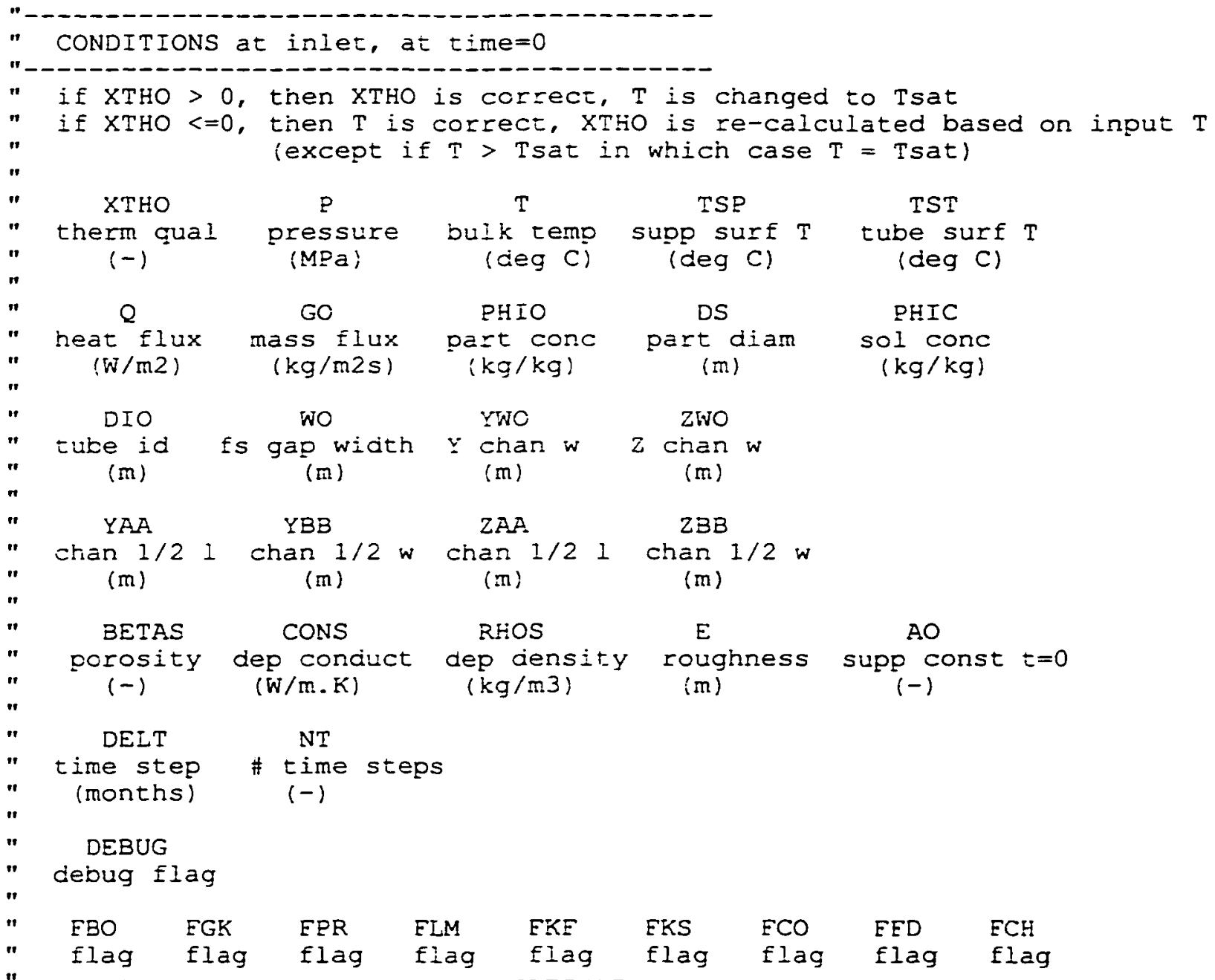




\section{APPENDIX E: TYPICAL OUTPUT FILE FOR TSFOUL}

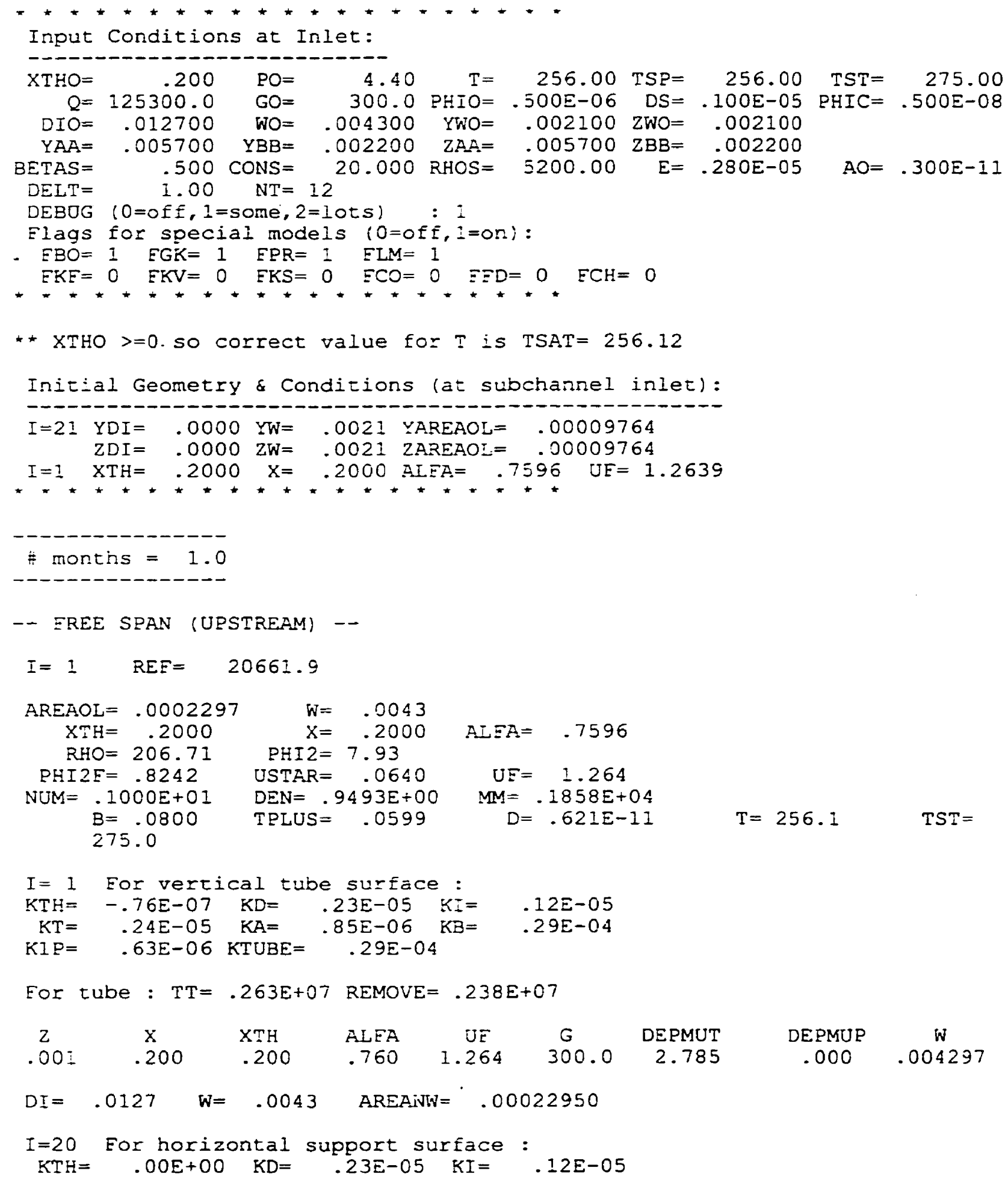




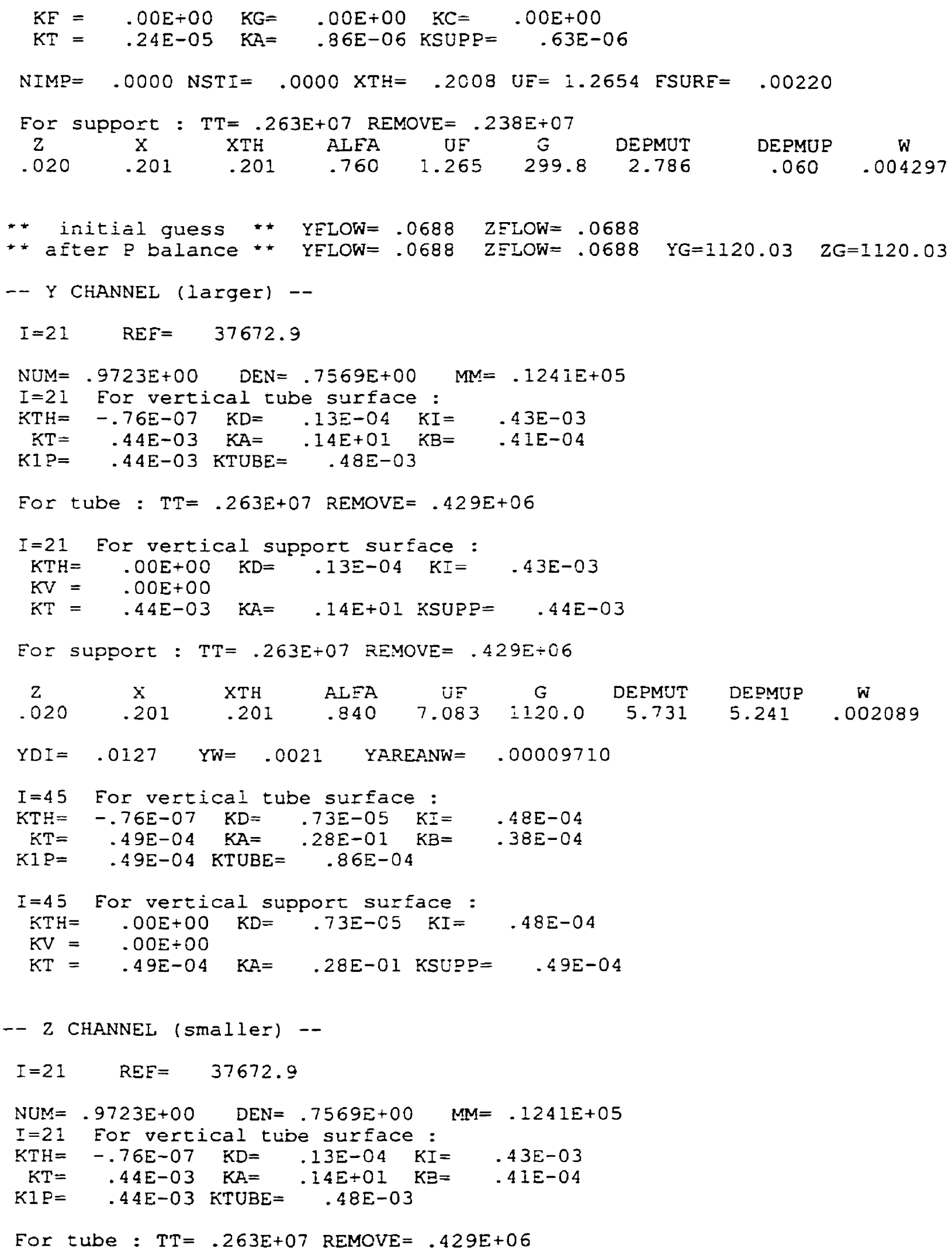




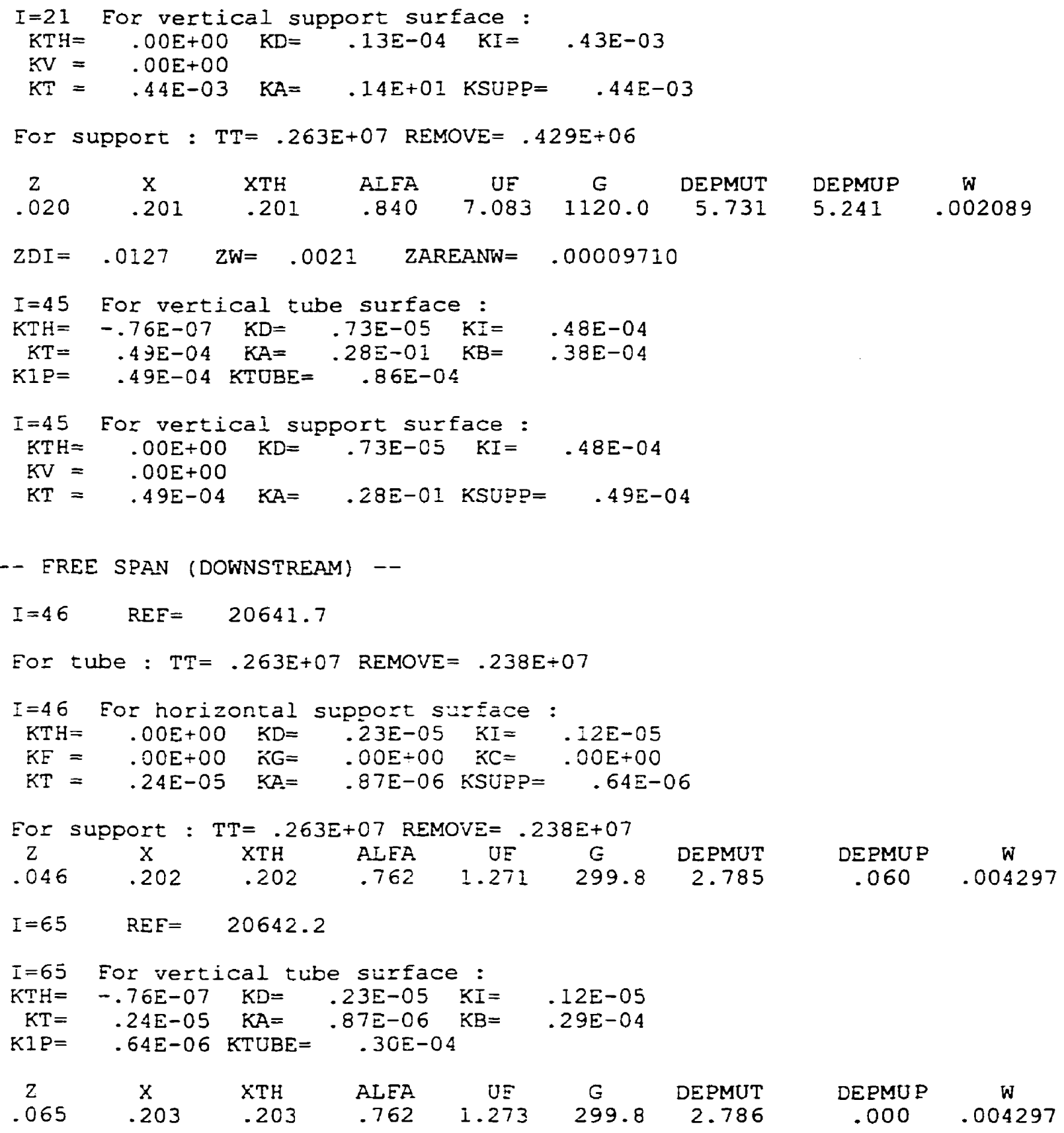

